\title{
Licensed NK cell responses to acute viral infection shape priming and differentiation of $\mathrm{CD8}+\mathrm{T}$ cells
}

Jeffrey Jun Hin Teoh

Katy, Texas

B.A. St. Olaf College, 2009

A dissertation presented to the Graduate Faculty at the University of Virginia in Candidacy for a Degree of Doctor of Philosophy

Department of Microbiology, Immunology, and Cancer Biology

University of Virginia

October 2016 


\section{Table of Contents}

\begin{tabular}{|c|c|}
\hline Abstract & iii \\
\hline Dedication & iv \\
\hline Acknowledgements & $\mathrm{V}$ \\
\hline List of Figures & vi \\
\hline Abbreviations & viii \\
\hline \multicolumn{2}{|l|}{ Chapter I: Introduction } \\
\hline Preface & 2 \\
\hline NK cells in antiviral immunity & 3 \\
\hline Host MHC I tunes NK responsiveness & 7 \\
\hline Licensed NK cells and viral infection & 14 \\
\hline NK-DC interactions & 16 \\
\hline CD8+ T cell immunity & 21 \\
\hline $\mathrm{CD} 8+\mathrm{T}$ cell differentiation & 24 \\
\hline CMV-specific CD8+ T cell responses & 30 \\
\hline NK cell influences on $\mathrm{T}$ cell immunity & 36 \\
\hline Significance \& Summary & 42 \\
\hline
\end{tabular}

Chapter II: Acute virus specific NK cell responses set CD8+ T cell dependence on CD27

Introduction 46

Results 49

Discussion _ 84

Chapter III: Virus specific NK cell responses shape CD8+ memory $\mathrm{T}$ cells and protective immunity

Introduction $\quad 89$

Results $\quad 92$

Discussion 127

\section{Chapter IV: General Discussion and Future Directions}

Introduction 
Licensed NK cell responses to viral infection influence the quality and duration of signals 1-3 for priming CD8+ T cells

Alternative co-stimulatory and inflammatory pathways 136

Extended duration of antigen exposure 142

Specific lymphoid compartment impacts on T cell priming $\quad 147$

Early MPEC skewing in mice with efficient licensed NK cell control of MCMV is IL-10 dependent

Quality versus quantity: The imprint of licensed NK cell-mediated resistance on CD8+ T cell memory

Longevity of licensed NK cell influence on T cell differentiation and maintenance of memory cells

Closing remarks 


\begin{abstract}
Natural killer (NK) cells represent a critical first-line of immune defense against a bevy of viral pathogens, and infection can provoke them to mediate both supportive and suppressive effects on virus-specific adaptive immunity. Previous studies have shown that licensed Ly49G2+ NK cells confer murine (M)CMV resistance in mice expressing MHC I D ${ }^{\mathrm{k}}$, a specific ligand of the inhibitory Ly49G2 receptor, which results in enhanced adaptive immunity due to accelerated accumulation of virus-specific CD8+ T cells. However, relatively little is known about the licensed NK-cell effect on T cell priming or ensuing effector and memory T-cell responses. We found that CD8+ T cell dependence on CD27 co-stimulation for early priming and differentiation is shaped by the efficiency of NK responses to virus infection. Moreover, T cell priming in the presence or absence of licensed NK cell control resulted in a profound skewing of CD8+ T cells into memory precursor effector cells (MPEC) or sustained short-lived effectors (SLEC), respectively. This T cell differentiation effect endured through viral latency such that the frequencies of memory and effector cells reflected differences observed for the strains at day 10. Transfer of virus-experienced CD8 $+\mathrm{T}$ cells from mice with or without licensed G2+ NK cells improved resistance against secondary challenge in naïve recipients. However, we observed distinct profiles and kinetics of resistance that likely reflected host-specific needs for distinct modes of $\mathrm{T}$ cell immunity. Thus specific virus control mediated by NK cells significantly impacts T cell priming, differentiation, and the manner in which $\mathrm{T}$ cells provide long-term protective immunity.
\end{abstract}




\section{Dedication}

This dissertation is dedicated to my family. I thank my parents, Ivan and Maureen Teoh, for their endless love, support, and encouragement in every facet of my life. I am grateful for all that they have taught me, including the values of hard work, determination, and at times, stubborn persistence. They have inspired and cultured my interests in the arts and curiosities in science, and have celebrated every step of my education and training. I am equally grateful to my five siblings - Deanna, Gillian, Bryan, Alison, and Katriana - and their spouses - Emil, Chris, and David. I am blessed to have grown up with such talented, creative, and ambitious siblings, whose shoes I have tried to fill in every day of my life. They have undoubtedly spurred a healthy competitive spirit in me, and perhaps more importantly, taught me the value of maintaining a good sense of humor in life. Finally, this thesis is dedicated to my daily partners in crime, Maggie, Chewie, and Loki. Our core unit has expanded over time, becoming furrier and funnier each and every day, and their unyielding love, encouragement, and inspiration has been essential to getting me through the trenches of graduate school. To Maggie in particular, I could not have done this without you. 


\section{Acknowledgments}

First and foremost, I would like to thank my mentor Dr. Michael G. Brown, whose unending patience, leadership, and enthusiasm in research has progressively shaped my scientific career. Curiosities in science are born from excellent mentors and colleagues; throughout graduate school, Mike's passion for advancing our understanding of the natural world and his meticulous application of the scientific method has been nothing short of inspiring. He has always challenged me to think about and perform the best science possible, and I am ever grateful for his mentorship.

Of course, I am also thankful for the guidance and continuous feedback from my thesis committee members, Drs. Thomas Braciale, Timothy Bullock, Alison Criss, Loren Erickson, and Ulrike Lorenz. They have offered diverse perspectives and insights that were critical to shaping the work described herein. In particular, their expertise in priming and differentiation of $\mathrm{T}$ cells has been invaluable, especially in the early stages of our ventures beyond NK cells. They have challenged me to think critically about my data and presentation of results. I could not have asked for a better team of scientists to evaluate, critique, and offer suggestions to further advance this work.

I would also like to acknowledge past and present members of the Brown laboratory, including Drs. Michael Stadnisky, Hairong Wei, William Nash, Pinar Pezük and Ebony Coats, Awndre Gamache, Alyssa Gillespie, Jessica Prince, Jack Cronk, and the undergraduates who have worked with us. It has been a privilege to stand on the shoulders of giants, and our current research would not be where it is today without everyone's hard work and contributions. Mike Stadnisky has been an outstanding mentor and friend: From day 1 of my summer rotation to the present, Mike S. has been a true role model and has been instrumental in teaching me flow cytometry, providing some of the initial discoveries for my research on T cells, and helping me develop my career. Alyssa Gillespie and Jessica Prince have been the lifeblood that keeps our lab running. Their technical and administrative expertise has been vital to the lab, and more importantly, their friendship and humor has cultivated a fun and inviting workplace. Lastly, words cannot express how much I cherish the brain trust that has emerged amongst William Nash, Awndre Gamache, and myself. Beyond their ready willingness to help with experiments and answer my many questions, Billy and Awndre are two of the most challenging yet insightful critics of all scientific work. Both are true scientists at heart, and I am excited to see where they push the field of immunology next. I cannot wait for our reunions to discuss and debate scientific results, wherever we may find ourselves.

Finally, I am indebted to the training and education that I have received at UVA. The MIC department and the Carter Immunology Center have greatly supported my research ventures and development as a scientist. I am grateful for our first-in class immunology courses, our weekly Research In Progress seminars, and the infectious disease training grant program, all of which have broadened my perspectives and critical thinking skills. I am also thankful for the UVA Flow Core and the training and leadership that Joanne Lannigan has provided to flow cytometry users at UVA. My time here has been a true pleasure. 


\section{List of Figures}

Figure 1.1 Integrated signals from activating and inhibitory NK cell receptors guide NK cell reactivity

Figure 1.2 Host MHC I tunes NK cell reactivity through tonic interactions with inhibitory NK receptors

Figure 1.3 Viral manipulation of the MHC I antigen processing and presentation

Figure 2.1 CD70 prompts accumulation of antiviral CD8+ T cells without impacting licensed NK cells responding to MCMV

Figure 2.2 Blockade of CD70 specifically impacts the proportion of CD8+ T cells

Figure 2.3 CD70 controls effector CD8+ T cell differentiation during acute infection in mice with licensed NK cell control of MCMV

Figure 2.4 CD8+ T cell dependence on CD70 is intact in CD4+ T celldepleted mice with licensed NK cell control of MCMV

Figure 2.5 CD70-dependent CD8 $+\mathrm{T}$ cell immunity proceeds in the absence of CD4+ T cells and licensed G2+ NK cell expression of CD40L 63

Figure 2.6 CD27-deficiency dampens virus-specific CD8+ T cell immunity without impairing licensed NK cell control of MCMV

Figure 2.7 CD27 is dispensable for licensed NK cell control of MCMV 70

Figure 2.8 Licensed NK cell control of MCMV adjust the duration of CD70 expression on DC

Figure 2.9 Inefficient NK-mediated control of MCMV results in delayed and CD27-independent accumulation of KLRG1+ SLECs 78

Figure 2.10 CD27 is critical for maintaining virus-specific CD8+ T cells after acute MCMV infection

Figure 3.1 Early viral control by licensed NK cells promotes memory precursor differentiation in virus-specific CD8+ T cells

Figure 3.2 Median fluorescence intensity of KLRG1 identifies two distinct $\mathrm{CD} 8+\mathrm{T}$ effector cell populations 
Figure 3.3 Licensed G2+ NK cell control of MCMV broadly impacts

differentiation of antigen-experienced cells

Figure 3.4 Scaled infection dose delays T cell accumulation but does not skew differentiation

Figure 3.5 Licensed NK cell enhancement of CD8+ T cell MPEC conversion is dependent on IL-10

Figure 3.6 IL-10R signaling decreases DC maturation and the prevalence of patrolling monocytes

Figure 3.7 Increased presence of circulating memory CD8+ T cells corresponds with efficient viral control during acute infection

Figure 3.8 Mice with licensed NK cell control of MCMV infection maintain greater numbers of central and effector memory CD8+ T cells into viral latency

Figure 3.9 MHC I-licensed G2+ NK cell responses to acute MCMV imprint on long term $\mathrm{CD} 8+\mathrm{T}$ cell differentiation skewing

Figure 3.10 CD8+ T cells transferred from mice without acute licensed NK cell control confer improved viral control to secondary challenge

Figure 3.11 Model of licensed NK cell influence on memory CD8+ T cell differentiation and distinct $\mathrm{T}$ cell responses to secondary challenge

Figure 4.1 Highly specific licensed NK cells influence CD8+ T cell priming through multiple pathways 


\begin{tabular}{|c|c|}
\hline & Abbreviations \\
\hline $\mathrm{Ag}$ & Antigen \\
\hline AIDS & Acquired immune deficiency syndrome \\
\hline APC & Antigen presenting cell \\
\hline$\beta 2 \mathrm{~m}$ & Beta-2 microglobulin \\
\hline B6 & C57B1/6 mouse strain (Black 6) \\
\hline CD62L & L selectin \\
\hline CMV & Cytomegalovirus \\
\hline Cmv1 & CMV resistance locus 1 \\
\hline d $\ldots$ & Days \\
\hline DAMP & Damage-associated molecular pattern \\
\hline DAP12 & K DNAX activation protein of $12 \mathrm{kDa}$ \\
\hline $\mathrm{D}^{\mathrm{k} / \mathrm{D}^{\mathrm{b}}}$ & MHC I D locus of the $\mathrm{k} / \mathrm{b}$ haplotype (mouse) \\
\hline $\mathrm{DC}$ & Dendritic cell \\
\hline Eomes & Eomesodermin \\
\hline FasL & Fas ligand \\
\hline Flt3 & Fms-like tyrosine kinase 3 \\
\hline G2 & Ly49G2 receptor (c571 allele, unless otherwise noted) \\
\hline GM-CSF & Granulocyte macrophage-colony stimulating factor \\
\hline $\mathrm{H}-2$ & Major histocompatibility complex (mouse) \\
\hline $\mathrm{HCMV}$ & Human cytomegalovirus \\
\hline $\mathrm{HCV}$ & Hepatitis $\mathrm{C}$ virus \\
\hline HIV & Human immunodeficiency virus \\
\hline HLA & Human leukocyte antigen (MHC I) \\
\hline HSV-1. & Herpes simplex virus-1 \\
\hline iDC & Immature DC \\
\hline IFN & Interferon \\
\hline ITAM & Immunoreceptor tyrosine-based activating motif \\
\hline ITIM & Immunoreceptor tyrosine-based inhibitory motif \\
\hline $\mathrm{K}^{\mathrm{k}} / \mathrm{K}^{\mathrm{b}}$ & MHC I K locus of the $\mathrm{k} / \mathrm{b}$ haplotype (mouse) \\
\hline KIR .... & Killer immunoglobulin receptor (human) \\
\hline KLRA & Killer cell lectin-like receptor, subfamily A \\
\hline $\mathrm{KO}$ & Knockout \\
\hline$-\mathrm{L} \ldots \ldots$ & -Ligand \\
\hline LCMV & Lymphocytic choriomeningitis virus \\
\hline Ly49 & Killer cell lectin-like receptor (mouse) \\
\hline $\mathrm{mAb}$ & Monoclonal antibody \\
\hline MA/My & Marsh albino Murray mice \\
\hline MCMV & Murine cytomegalovirus \\
\hline MHC I/II & Major histocompatibility complex class I/II \\
\hline MIP- $-\alpha / \beta$ & Macrophage inflammatory protein $\alpha / \beta$ \\
\hline MPEC & Memory precursor effector cell \\
\hline NK & Natural killer \\
\hline $\mathrm{NKC}$ & Natural killer cell gene complex \\
\hline OT-I & $\mathrm{CD} 8+\mathrm{T}$ cell with a transgenic $\mathrm{TCR}$ specific for $\mathrm{OVA}_{2}$ \\
\hline
\end{tabular}


OVA $_{257} \ldots$ Ovalbumin 257-264 peptide; SIINFEKL

PAMP Pathogen-associated molecular pattern

PBMC Peripheral blood mononuclear cell

pDC - Plasmacytoid DC

-R $\quad$-Receptor

SHP-1/2 Src homology region 2 domain containing phosphatase- $1 / 2$

SLAM Signaling lymphocyte activation molecule

SLEC Short-lived effector cell

T-bet $\quad$ T-box transcription factor, encoded by $T b \times 21$

$\mathrm{T}_{\mathrm{CM}} \quad \mathrm{T}$ central memory cell

$\mathrm{T}_{\mathrm{EM} \ldots \ldots \ldots . . . \quad \mathrm{T} \text { effector memory cell }}$

TCR-p:MHC TCR-peptide-MHC I

TLR Toll-like receptor

TCR - T cell receptor

TNF Tumor necrosis factor

TRAIL $\quad$ TNF-related apoptosis-inducing ligand

VV Vaccinia virus

WT _.......... Wild type 


\section{Chapter I}

\section{Introduction*}

* Written excerpts and figures from Nash et al. Frontiers in Immunology (2014) were used within Chapter I. J Teoh authored the text and figures within. 


\section{Preface}

Natural killer (NK) cells and CD8+ T cells are critical antiviral effector cells of the innate and adaptive immune responses to infection, respectively. While both cell types host an arsenal of cytokines and cytotoxic granules to kill infected target cells, unique kinetic profiles manifest as a result of their distinct target cell recognition mechanisms. NK cells are guided by a series of signals through germline encoded activating and inhibitory receptors on their surface, which allows them to survey targets without need for prior sensitization (1). In contrast, CD8 + T cells are clonal in nature, and require more time to traffic through lymphoid organs to be primed by antigen presenting cells in conjunction with co-stimulatory signals to promote $\mathrm{T}$ cell immunity over tolerance (2). In addition to kinetic differences, NK and CD8+ T cells display nonoverlapping functions for restricting certain viral infections (3-5). Moreover, the shear diversity of viral evasion genes specific to NK cells and T cells further highlights nonredundant roles of these cell types for effective immune control $(6,7)$.

Although conventional characterization of innate and adaptive immune responses has focused predominantly on temporal separation of peak cellular activities, it is now well appreciated that innate cell sensing of foreign pathogens informs the quality, type, and magnitude of adaptive responses to infection (8). While a role for PAMP sensors in driving adaptive immunity has been recognized for some time (9), the immunoregulatory functions of NK cells have only been recently described. Certainly, frontline defenses provided by NK cells have been recognized to yield significant influence on the extent of early viral replication and virus-mediated tissue pathology, which can instruct the magnitude and efficiency of $\mathrm{T}$ cell priming $(2,4,10)$. Recent studies have uncovered 
additional influences of NK cells on adaptive immunity, including regulation of DC maturation, inflammatory and anti-inflammatory cytokine production, and direct cytolysis of activated $\mathrm{T}$ cells during certain infections $(11,12)$. This ability for NK cells to modulate $\mathrm{T}$ cell immunity has garnered much attention, particularly as we consider new strategies for immunotherapeutic interventions to increase the efficacy of antiviral or anti-tumor CD8+ T cells.

Despite that the role of NK cells in shaping adaptive CD8+ T cell immunity is now widely accepted, it remains a subject of intense debate as to whether NK cells support or suppress T cell performance. Several studies have identified NK-mediated restriction on adaptive immunity, such that depletion of NK cells can augment the magnitude and performance of virus-specific $\mathrm{CD} 4+\mathrm{T}, \mathrm{CD} 8+\mathrm{T}$, and germinal center $\mathrm{B}$ cells (13-19). Other groups have identified critical functions for NK cells in stimulating DC maturation and CD8 $+\mathrm{T}$ cell accumulation early after infection or disease detection (20-24). This dichotomy of responses is to be expected across genetically distinct hosts and various infections. Developing a stronger understanding for how different NK responses to infection affect the spectrum of influence on $\mathrm{T}$ cell immunity will be invaluable as we seek to improve targeted immunotherapies.

\section{NK cells in antiviral immunity}

NK cells comprise up to $15 \%$ of total circulating lymphocytes $(2-3 \%$ in the spleen) and can be found throughout lymphoid and non-lymphoid tissues (25). Armed with an arsenal of cytotoxic proteins (perforin, granzyme) and death ligands (FasL, TRAIL), NK cells can kill their targets on contact without the need for prior sensitization, 
which aptly defines their namesake. Additionally, NK cells are well known for their robust production of inflammatory cytokines including IFN $\gamma$ and TNF $\alpha$, as well as the maturational cytokine, GM-CSF, and the chemokines MIP- $1 \alpha$, and MIP-1 $\beta$. Unlike their adaptive lymphocyte counterparts, NK cells constantly survey host tissue for indications of stress or damage using a combination of germ-line encoded inhibitory and activating receptors (26). Although NK cells lack somatic re-arrangement of their receptors to account for the range of epitopes for which $\mathrm{T}$ and $\mathrm{B}$ cells recognize, they have developed alternative mechanisms to detect a diverse variety of target cells. NK receptors are derived from an extremely polygenic and polymorphic gene complex $(27,28)$. By encoding several distinct polymorphic receptors, NK cells are equipped with an extensive repertoire for recognizing polymorphic ligands. Moreover, these receptors are expressed by individual NK cells in a stochastic and variegated manner, such that varied constellations of inhibitory and activating receptor expression endow NK cells with multiple strategies to discern healthy cells from cells that are in distress (29).

NK cells were first described in the early 1970's for their ability to lyse syngeneic and allogeneic tumor cells (30). Perhaps one of the most seminal discoveries was the ability for NK cells to detect and respond to the absence of self-major histocompatibility complex class I (MHC I) expression on select tumor cells (31). This discovery gave rise to the 'missing-' or 'altered-self' hypothesis for NK-mediated target cell recognition, and clarified how NK cells could detect virus-infected cells or tumor cells that downregulated MHC I as a means to escape CD8+ T cell detection. Furthermore, it was appreciated that NK cells are not restricted to peptide-MHC complexes in the same 
fashion as CD8+ T cells, and could thus provide unique host-protective immune functions.

NK cell responses have been well characterized beyond their reactivity to select tumor cells, and are perhaps better known for their critical frontline protection against viral infections. Early in vitro experiments with a variety of viral infections (vesicular stomatitis virus, influenza (H3N2 and A/PR/8/35), vaccinia virus (VV; Lister strain), measles, and herpes simplex virus-1 (HSV-1)) and direct administration of type I IFN to lymphocyte co-cultures with adherent cell lines demonstrated enhanced cytotoxic activities of human NK cells $(32,33)$. By ruling out the contribution of $\mathrm{T}$ and $\mathrm{B}$ lymphocytes, the abovementioned studies helped establish an early antiviral role for NK cells. Numerous other groups have also reported important NK cell responses to viral infections (reviewed in (34)). Moreover, in humans, classical or functional deficiencies in NK cells are often associated with increased susceptibility to a bevy of viral pathogens $(35-37)$.

Perhaps the most convincing demonstration for critical NK-mediated resistance to viruses has been with primary herpesvirus infections, including Epstein-Barr virus, HSV1, varicella zoster virus, and most notably, cytomegalovirus (CMV) $(35,37)$. Early investigations of host resistance to murine (M)CMV in experimental inbred mouse strains uncovered several genetic determinants of resistance that could be attributed to both MHC I and non-MHC I genes (38-41). The above studies also observed positive correlations between the degree of NK cell cytotoxicity with the level of MCMV resistance in various inbred strains, which suggested that NK cells were critical mediators of early antiviral immunity (40). In support of their role in controlling CMV infection, 
NK cell deficiencies in humans and mice were found to correspond with increased vulnerabilities to $\mathrm{CMV}$, even when adaptive $\mathrm{T}$ and $\mathrm{B}$ cell immune components were intact $(3,4,35,42)$. Recent studies have highlighted that CMV infection in humans and mice leave stable imprints on the frequencies and phenotypes of specific NK cell subsets, with the most virus-responsive NK subsets accounting for the majority of the persistent expanded populations $(43,44)$.

Additional evidence of NK cell contributions to antiviral immunity stems from the evolutionary selection of NK receptors that can specifically detect virus-infected cells. Through comparisons of the naturally MCMV-resistant C57BL/6 (B6) and susceptible BALB/c inbred mouse strains, it was discovered that a dominant MCMV resistance factor, $\mathrm{Cmvl}$, conferred efficient viral control independent of the MHC-I haplotype (45), and mapped to the NK gene complex (NKC) on chromosome 6 (46). Further genomic study of the $\mathrm{Cmvl}$ locus identified the key resistance gene as Klra8, or $L y 49 h$, which is an NK cell activating receptor that can specifically detect MCMVinfected cells that express the virus-derived m157 ligand (47-49). While this particular resistance gene is not necessarily common amongst inbred and wild mouse populations (50) - likely due to viral escape mutations eliminating or altering expression of the $\mathrm{m} 157$ ligand (51) - this finding has nonetheless demonstrated the degree of virus-specificity to which NK cells can respond. Additional virus-specific activating receptors have since been identified in certain strains of mice for a variety of other infections, including ectromelia virus (52) and HSV-1 (53). Moreover, combinations of specific inhibitory NK receptors with defined MHC I haplotypes have also been shown to improve antiviral NK cell activities (54) [Discussed in detail below]. 
Thus, it is clear that NK cells are critical antiviral defenders in host immunity and that specific mechanisms of NK recognition and response to infected target cells have likely co-evolved with a variety of viral pathogens. Despite their classification as innate immune cells, there is remarkable diversity in NK receptor expression between genetically distinct hosts, which impacts how they interact with their host environment. While this diversity underscores the importance of NK cells in mediating resistance to a variety of viral pathogens, it also highlights the range of impacts that NK cells can have on host immunity and the potential for differences in immunoregulation by NK cells.

\section{Host MHC I tunes NK responsiveness}

As innate defenders against viruses, NK cells are in a constant state of poise to discern healthy from infected or stressed cells. Upon target recognition, the balance between immunity and tolerance is guided by integrated intracellular signals from activating and inhibitory receptors (Fig. 1.1). Amongst these receptors are the killerimmunoglobulin receptors in humans (KIR) and the C-type lectin receptors in mice (Ly49), both of which can either be inhibitory or activating (55). Inhibitory receptors bind MHC I as a ligand, and are characterized by their long intracellular tails, which include immunoreceptor tyrosine-based inhibitory motifs (ITIM). When stimulated they can recruit tyrosine phosphatases such as SHP-1 and SHP-2 to dampen NK activation (56). On the other hand, activating receptors have shorter intracellular tails, and are associated with the adaptor molecule DAP12 that contains an immunoreceptor tyrosinebased activating motif(ITAM). When activating receptors are stimulated, they signal through DAP12 to recruit tyrosine kinases and promote NK activation. Ligands for 
activating KIR or Ly49 include MHC I as well as certain viral proteins (56). Thus at a rudimentary level, the NK responses are decided by the balance between activating and inhibitory signals. Whereas abundant expression of MHC I on a healthy host cell will inhibit NK cell activation, altered or missing expression of MHC I or an overwhelming presence of stress-inducible ligands on infected cells or tumor cells will provoke immunoreactivity (1).

In addition to the instantaneous snapshot of NK receptor interactions with target cells, NK responsiveness is further tuned by tonic interactions with host-MHC I through a process known as 'licensing' $(54,56,57)$. This phenomenon was first recognized by the fact that $\beta 2 \mathrm{~m}$-deficient mice (i.e. MHC I deficient mice) do not exhibit signs of NKmediated autoimmunity despite exhibiting normal numbers of mature NK cells (58). Similarly, NK cells lacking self-specific inhibitory receptors can also achieve selftolerance in the absence of inhibition. Although somewhat paradoxical, it has been demonstrated in both humans and mice that NK cells without prior inhibition can achieve self-tolerance through becoming hyporesponsive, and are regarded as 'unlicensed.' In contrast, NK cells that are habitually inhibited by self-MHC I attain self-tolerance through tonic inhibitory signaling; these cells are 'licensed,' and exhibit functionally competent responses to activating stimuli when inhibition is released or overcome (Fig. 1.2A-C) (59-61).

While certain molecular components of NK licensing, such as SHP-1, have been described previously (62), the precise mechanisms and molecular signatures that guide and define licensed NK cells have yet to be determined (56). Nonetheless, much has been learned about the process and elements of licensing NK cells: (i) Licensing is not a 
binary event that determines the ability of NK cells to respond. Rather it is a tunable process for each inhibitory receptor-MHC I pair, such that the degree of resting inhibition determines NK cell sensitivity to changes in the expression of MHC I $(63,64)$. The extent of inhibition is not only influenced by the total number of unique receptor-ligand interactions, but also by the specific density of expression of both inhibitory receptors and cognate MHC I ligands (65). (ii) The relative licensing status of an NK cell is plastic, and is subject to change depending on the microenvironment. Transferring mature NK cells between MHC-disparate hosts can reset the functional competence of cells - to licensed or unlicensed - within a matter of days (Fig. 1.2D) (66-68). Selectively inducing host expression of MHC I can similarly endow NK cells with improved licensed responses to activating stimuli (69). Similarly, MHC I ${ }^{\text {low }}$ tumor microenvironements can also reduce functional tumor-infiltrating NK performance (70). (iii) Finally, optimal NK cell licensing conditions during development require cognate MHC I expression on both hematopoietic and non-hematopoietic cells $(68,71,72)$. Restriction of MHC I expression to either hematopoietic or non-hematopoietic compartments can reduce NK cell rejection of MHC I-deficient targets as well as NKmediated resistance to viral infection. 
Figure 1.1. Integrated signals from activating and inhibitory NK cell receptors guide NK cell reactivity. NK cells discern healthy from stressed cells through their variegated expression of polymorphic activating and inhibitory NK receptors. Responsiveness to target cells is directed by the integration of proximal receptor signals that are triggered upon receptor stimulation. Activating receptors have a short intracellular tail, and are paired with the adaptor signaling protein DAP12, which features an immunoreceptor tyrosine-based activating motif (ITAM). The ITAM can recruit and phosphorylate activation-linked signaling molecules (Vav, $\left.\mathrm{PIP}_{2}\right)$, which are critical to NK cell activation. In contrast, inhibitory receptors have a long intracellular tail that features an immunoreceptor tyrosine-based inhibitory motif(ITIM). The ITIM can recruit phosphatases such as SHP-1/-2, and SHIP-1 for dephosphorylation of proximal activation signaling molecules such as phosphorylated Vav or $\mathrm{PIP}_{3}\left(\rightarrow \mathrm{PIP}_{2}\right)$. Inhibitory receptors can also induce phosphorylation of the small adaptor molecule, Crk, which causes Crk to associate with c-Abl and sequesters this adaptor protein from the activation-signaling complex. 
Figure 1.1

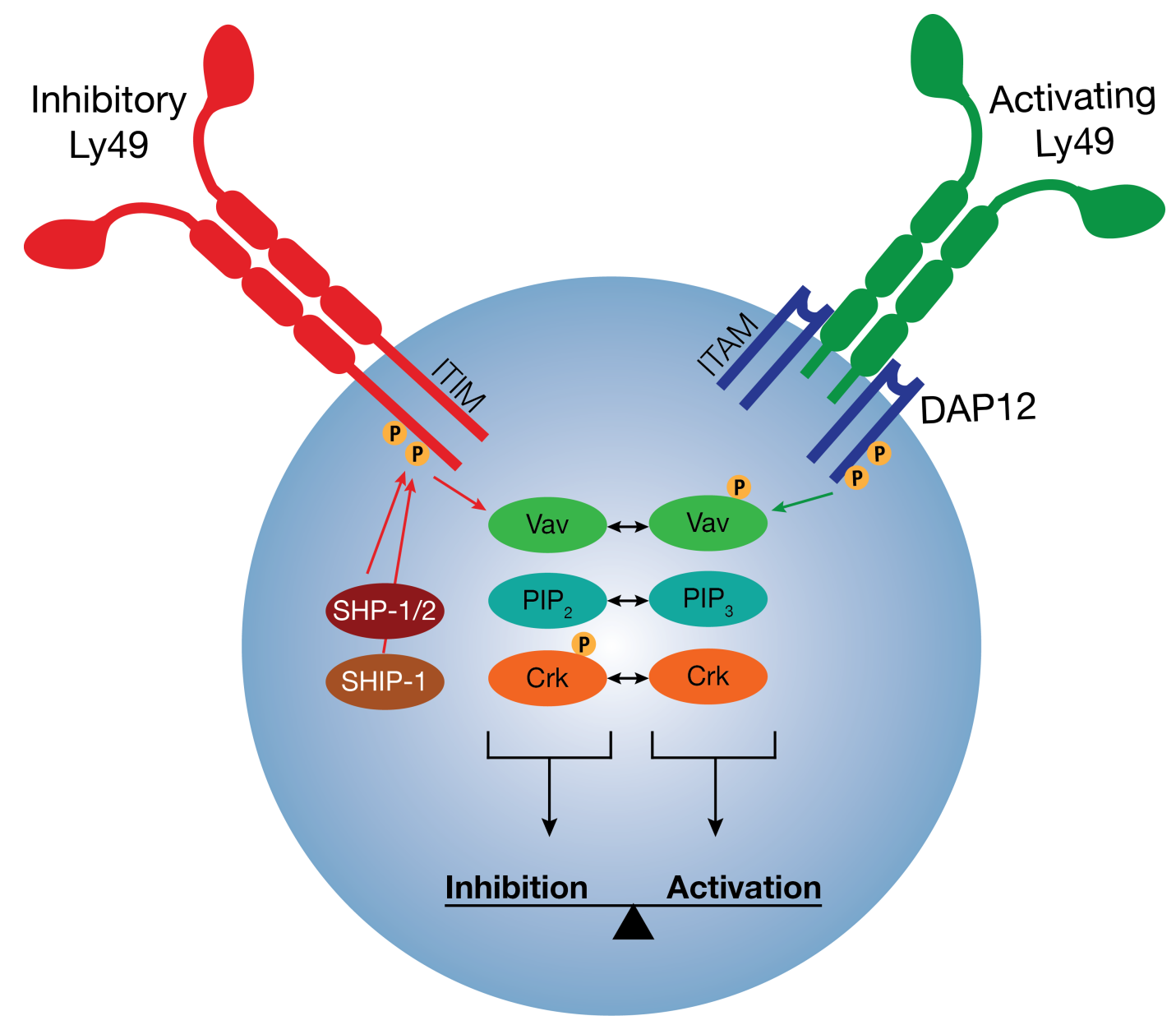


Figure 1.2. Host MHC I licenses NK cells through tonic interactions with inhibitory receptors. Resting MHC I interactions with NK inhibitory receptors confer enhanced NK cell responsiveness to $\mathrm{MHC} \mathrm{I}^{\text {low }}$ target cells through a process known as 'licensing.' NK cells expressing at least one self-specific inhibitory receptor for self-MHC I ligand are tuned to detect changes in MHC I expression rather than the sole absence of MHC I. $(\mathrm{A}, \mathrm{B})$ In the absence of either cognate MHC I ligand or self-specific inhibitory receptors, NK cells are unlicensed and demonstrate reduced cytotoxicity towards MHC I ${ }^{\text {low }}$ target cells. (C) NK cells that express self-specific inhibitory receptors in environments with their cognate MHC I ligand expressed are licensed, and mediate efficient lysis of target cells upon loss of MHC I. (D) Licensing is a dynamic and plastic process. Transfer of previously unlicensed NK cells into an environment where inhibitory receptors can interact with cognate MHC I ligands results in re-licensing of NK cells. 
Figure 1.2

Host MHC I

Environment

A

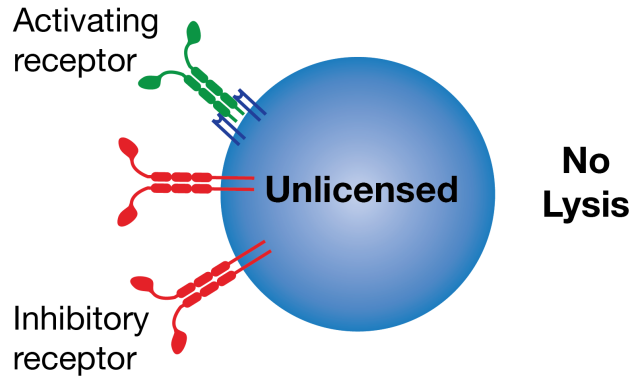

\begin{tabular}{c}
$\begin{array}{c}\text { Target } \\
\text { Cell }\end{array}$ \\
\hline
\end{tabular}

Absence of MHC I

receptor
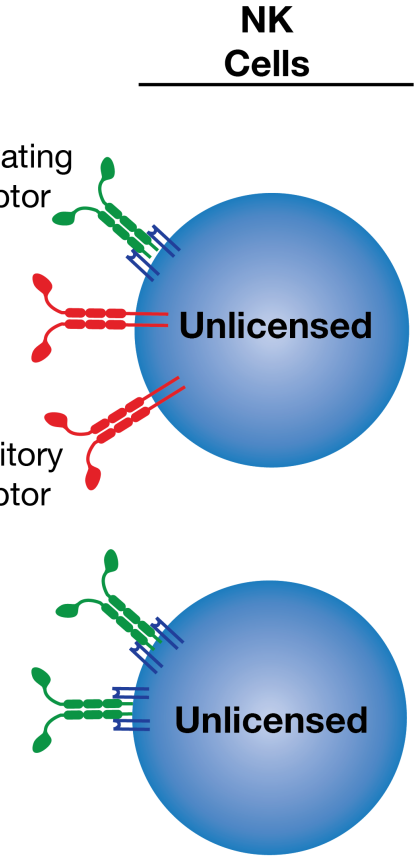

B

MHC I

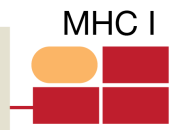

No

Lysis

C

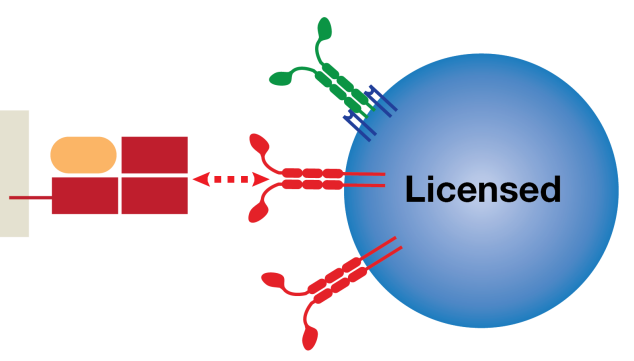

Lysis

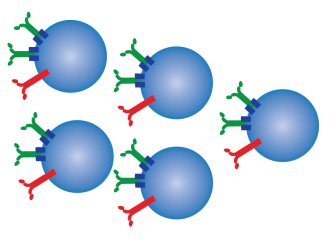

No

\section{Unlicensed}

Lysis MHC I

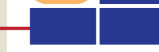

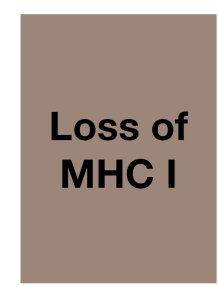

Loss of

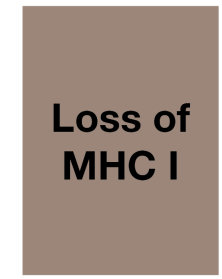

\section{Loss of} MHC I

MHC I

Adoptive transfer of mature NK cells

\section{Cognate} MHC I

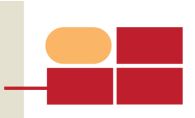

Lysis

Loss of MHC I

Re-licensed 


\section{Licensed NK cells and viral infection}

While licensing through most inhibitory receptors can establish a general state of functional effector cell tolerance to self, certain MHC I ligand pairings with inhibitory receptors have been correlated with improved host resistance to specific viral infections. In humans, this is most notable in HIV infection, where patient expression of inhibitory KIR3DL1 and human leukocyte antigen (HLA)-Bw4 corresponds with delayed disease progression to AIDS (73). Previous studies have further noted specific expansions of NK subsets expressing inhibitory KIR3DL1 in the peripheral blood of HIV+ patients when HLA-Bw4 was also expressed (74). Moreover, in vitro investigations of NK cell cytotoxicity against autologous CD4+ T cells infected with HIV demonstrated that the magnitude of target cell rejection corresponded with the degree of licensing as determined by KIR3DL1 and HLA-Bw4 expression density (65). Likewise, favorable pairings of inhibitory NK receptors with HLA subtypes have also been reported for improved resistance to hepatitis C virus (HCV), influenza, and VV (75).

Inbred mouse strains display variable resistance to experimental infections, and have been useful for exploring the influence of MHC I (H-2) haplotypes on the responsiveness of NK cells with a given repertoire of receptors. These receptor repertoires are genetically encoded in the highly polymorphic $\mathrm{NKC}$, which varies between inbred mouse strains. As mentioned previously, early characterizations of inbred strain resistance to MCMV infection revealed MHC I as well as non-MHC I linked resistance factors (38-41). Although the Ly49H NK activating receptor provides enhanced antiviral protection in B6 mice, $\mathrm{H}-2$ linked resistance is a prominent determinant of viral protection amongst a number of other strains. Notably, $\mathrm{H}-2^{\mathrm{k}}(\mathrm{k}$ 
haplotype of MHC I) has long been associated with improved host resistance to MCMV infection for a number of strains, including $\mathrm{CBA}$ and $\mathrm{C} 3 \mathrm{H} / \mathrm{HeN}$ mice $(38,41)$. Marsh albino Murray (MA/My) mice also express $\mathrm{H}-2^{\mathrm{k}}$ and demonstrate robust NK-dependent antiviral responses to MCMV infection (76). Utilizing classical genetic techniques to compare the extent of MCMV resistance in MA/My mice $\left(\mathrm{H}-2^{\mathrm{k}}\right)$ and more susceptible C57L $\left(\mathrm{H}-2^{\mathrm{b}}\right)$ or BALB/c mouse strains $\left(\mathrm{H}-2^{\mathrm{d}}\right)$, our lab and others have determined that the MHC I $D^{k}\left(D^{k}\right)$ locus, but not MHC I-K ${ }^{k}$, is critical to NK-mediated MCMV resistance (77-79). We also demonstrated that transgenic expression of $\mathrm{D}^{\mathrm{k}}$ in $\mathrm{C} 57 \mathrm{~L}$ mice confers improved viral resistance (71).

NK subset-specific depletion studies in mice with $\mathrm{D}^{\mathrm{k}}$ have identified that NK cells expressing inhibitory receptor Ly49G2 (G2) are critical for mediating enhanced resistance $(71,78)$. Interestingly, $\mathrm{D}^{\mathrm{k}}$ is a cognate ligand for the inhibitory $\mathrm{G} 2$ receptor $\left[\mathrm{Ly} 49 \mathrm{G} 2^{\mathrm{c} 571, \mathrm{ma} / \mathrm{my}}\right.$ alleles; Ly49G2 $2^{\mathrm{b} 6}$ does not bind to $\left.\mathrm{D}^{\mathrm{k}}\right](80)$, and can thus license G2+ NK cells for enhanced cytotoxicity in response to activating receptor stimulation as well as MHC $\mathrm{I}^{\text {low }}$ target cells $(68,71)$. Moreover, we observe specific expansion and accumulation of $\mathrm{G} 2+\mathrm{NK}$ cells in response to MCMV in mice with the $\mathrm{D}^{\mathrm{k}}$ ligand expressed, but not when the ligand is absent or restricted to specific tissue compartments. Whether these licensed G2+ NK cells are responding largely to subtle changes in $\mathrm{D}^{\mathrm{k}}$ expression or whether their reactivity is paired with another activating signal that is augmented by the licensing process remains to be determined.

Genetic screens and in vitro studies have suggested a role for activating receptor Ly49P mediating $\mathrm{D}^{\mathrm{k}}$-dependent resistance to $\operatorname{MCMV}(79,81)$. Recognition and response to infected cells by Ly49P required expression of $\mathrm{D}^{\mathrm{k}}$ in complex with MCMV-derived 
protein $\mathrm{m} 04$, however the importance of Ly49P in vivo has not been verified due to the lack of Ly49P monospecific depleting antibodies (54). Nonetheless, our studies have demonstrated an important role for inhibitory G2 receptor pairing with its cognate $\mathrm{D}^{\mathrm{k}}$ ligand in mouse resistance to MCMV.

Despite the robust level of immunity conferred by $\mathrm{D}^{\mathrm{k}}$-licensed G2+ NK cells during MCMV infection in certain strains, it is still debated whether licensing improves or hinders NK-mediated immunity. Studies of Ly49H+ NK-mediated immunity in B6 mice demonstrate that co-expression of self-specific inhibitory receptors with Ly49H can actually limit proliferative bursts and persistence of MCMV-specific NK cells, relative to NK cells without self-specific inhibitory receptors expressed (82, 83). During influenza infection, NK cells expressing inhibitory receptors can be functionally inhibited by viral clustering of MHC I ligands (84). Moreover, NK cells without self-specific inhibitory receptors (and even NK devoid of all Ly49 receptors) demonstrate improved perforindependent mechanisms of viral control (85). Stable imprints of CMV infection on human NK cells are prominently characterized by the expression of activating KIR and lectinreceptor NKG2C, although self-specific inhibitory KIR are also commonly co-expressed (43). Hence, the perceived benefits of inhibitory receptor licensing to NK cell performance against a viral infections are likely to vary based on the particular combinations of MHC ligands and NK receptors expressed as well as the nature of the viral pathogen.

\section{NK-DC interactions}


Whereas NK cells are patrolling effector cells constantly poised to detect and defend against infected or cancer cells, dendritic cells (DCs) are professional antigen presenting cells that act as sentinels to sample and present antigen to T cells. Most phagocytic cells are known for their abilities to engulf and present antigen via MHC class II (MHC II) molecules. However, DCs additionally have the ability to cross-present exogenous antigen through MHC I, which allows them to efficiently prime CD8+ T cells (86). DC exposure to antigen - either local or systemic - results in antigen uptake, processing, and presentation to T lymphocytes (87). Antigen presentation alone, however, is not sufficient to stimulate $\mathrm{T}$ cell immunity. DCs regularly sample antigen in the periphery as a result of cellular turnover and subsequently traffic to lymphoid organs where they present antigen to CD4+ $\mathrm{T}$ and $\mathrm{CD} 8+\mathrm{T}$ cells. Despite this process, autoimmunity against peripheral tissues is not observed, and peripheral tolerance is maintained via deletion of self-reactive $\mathrm{T}$ cells and induction of regulatory $\mathrm{T}$ cells (88). When DCs acquire antigen under inflammatory conditions, however, phenotypic and functional maturation of DCs can ensue (characterized by increased surface expression of co-stimulatory ligands and improved production of inflammatory cytokines, respectively) and interactions with $\mathrm{T}$ cells can now promote immunity over tolerance (88-90). Thus, in addition to surveying antigen, DCs function as critical sentinels that bridge the gap between innate and adaptive immune responses.

NK cells efficiently respond to infected- or tumor target cells, and thus influence both the amount and type of antigen available for DC uptake and the degree of DC maturation. As a demonstration of this potential, Hoebe and colleagues transferred varied amounts of MHC I-deficient target cells that expressed an immunogenic peptide as a 
minor histocompatibility antigen into MHC I replete mice (91). NK cell rejection of the transferred target cells released immunogen, which was then taken up by DCs and used to prime CD8 $+\mathrm{T}$ cell responses in a dose-dependent manner. Accordingly, NK-mediated apoptosis of the target cells was sufficient to increase inflammatory signaling and lead to the maturation of DCs $(91,92)$. Similarly, in a mouse model of influenza infection (PR8), NK cells were demonstrated to promote efficient DC and T cell recruitment and priming in the mediastinal lymph nodes via two non-redundant mechanisms: (i) NK cell IFN $\gamma$ was demonstrated to be critical for the up-regulation of chemokines CXCL10 and CCL21, both of which are required for efficient recruitment of T cells and DCs. (ii) NK release of perforin was necessary to induce apoptosis of infected cells, which would release antigen in the lung for uptake and presentation by DCs (93). Finally, for infections that occur in secondary lymphoid organs, the efficiency of NK cell responses can impact lymphoid organization, which can have direct impacts on how DCs interact with $\mathrm{T}$ cells $(10,94)$. These studies demonstrate how the kinetics of NK responses to damaged- or diseased tissues can influence the initiation of adaptive immunity.

Whereas the impact of NK cells on available antigen and tissue integrity may be viewed as a byproduct of overlapping arms of immunity, direct NK-DC interactions have previously been observed with bidirectional effects on both NK and DC immunity. The earliest indication of direct NK-DC interactions came from studies that examined the ability of fms-like tyrosine kinase-3 (Flt3) ligand expanded DCs to improve NK cell rejection of solid tumors in athymic nude mice (95). In that study, in vitro co-cultures of DCs with NK cells further confirmed that cell-to-cell contact was required for optimal enhancement of NK cell performance. Numerous studies have identified key signaling 
mediators that are exchanged via direct cell-to-cell interactions or across tight immune synapses $(95,96)$. These molecules include IL-12, IL-15, IL-18, and type I IFN, all of which can influence NK cell activation, survival, proliferation, and cytokine release (97, 98).

NK cells can also provide specific maturation signals to DCs through both soluble and contact-dependent means. Co-cultures of fresh or IL-2 activated NK cells with immature DCs (iDCs) were shown to increase DC production of IL-12 and expression of co-stimulatory ligands (CD80, CD86, HLA-DR) $(99,100)$. Interestingly, the maturation effects of NK cells on immature DCs (iDCs) are dependent on the ratio of NK cells to iDCs, with low ratios promoting positive maturation of DCs and high ratios inhibiting NK-mediated maturation or even killing of iDCs (100). Removal of iDCs by NK cells can promote immunity by selecting for DCs that are most likely to prime rather than tolerize naïve antigen-specific T cells. This process of DC editing has been previously described (101), and may have important implications for understanding how certain chronic viral infections, such as HIV, interfere with NK-DC crosstalk in order to evade adaptive immunity (102).

It is worth noting that during MCMV infection NK cells and DCs can occupy similar spaces in the spleen. Splenic stromal cells lining the marginal zone are amongst the first and most prominent cells to become infected $(103,104)$. Within $48-96$ hours following infection, both DCs and NK cells line the marginal zone $(10,94)$, presumably to sample antigen and to lyse infected cells, respectively. Thus the potential for these two cell subsets to interact with one another is relatively high. NK cell-mediated killing of infected stromal cells could provide abundant antigen for the DCs, and likewise, cytokine 
feedback loops between the two cells (IFN $\gamma$, IL-12, IL-15, type I IFN, etc.) could amplify NK activation and DC maturation. While this has yet to be formally demonstrated in vivo, it has been previously shown that $\mathrm{CD} 8 \alpha+\mathrm{DCs}$ are required to aid the proliferative burst of MCMV-specific Ly49H+ NK cells in an IL-12 and IL-18 dependent manner (105).

Interestingly, the efficiency of NK cell-mediated resistance to acute MCMV infection corresponds with the preservation and recovery of splenic DCs, which consequently impacts the ability to prime antiviral $\mathrm{CD} 8+\mathrm{T}$ cell responses $(23,24,105$, 106). That being said, the ultimate cause for a loss of DCs during MCMV infection has yet to be determined. In a study by Robbins et al. it was shown that efficient viral control by Ly $49 \mathrm{H}+\mathrm{NK}$ cells could restrict the early production of type I IFN $\alpha / \beta$ by plasmacytoid DCs (pDCs), which was posited to be directly toxic to DCs during infection (23). However, recent studies have challenged this notion of type I IFN toxicity to DCs. Type I IFNa receptor (IFNAR) deficient mice demonstrated increased levels of MCMV infection in DCs, which induced a state of functional paralysis characterized by unstable expression of MHC I and a general downregulation of surface co-stimulatory molecules. Moreover, neither IFNAR deficiency nor pDC depletion is sufficient to protect against specific loss of DCs during MCMV infection [Nash et al. Submitted]. Thus, alternative mechanisms of DC loss are likely. DCs can be targeted for infection by MCMV, and it is possible that NK-mediated elimination accounts for the drastic DC loss (107-109). However, depletion of total NK cells during infection has thus far proven insufficient to rescue DC numbers (24) [Nash et al. Submitted]. Several groups have confirmed that while DC infection is possible, the number of MCMV-infected DCs in vivo is relatively 
minimal, which makes NK-mediated elimination unlikely $(103,104,106,110,111)$. Another possibility is that under extreme viral duress, DCs traffic out of the spleen to prime $\mathrm{T}$ cells in alternative lymphoid organs, but there is currently little evidence to support this outcome [Nash et al. Submitted]. Regardless of the cause for DC loss during MCMV infection, what is clear is that NK cells can mediate improved DC maintenance and/or rescue, and this can yield substantial impacts on adaptive immune responses.

Whether NK cell impacts on DCs during MCMV infection are direct via cell-tocell interactions or indirect through viral control remains to be clarified. Infecting susceptible mouse strains with low dose MCMV infections or engineered MCMV strains that can be induced to arrest viral replication has demonstrated improved DC recovery and preservation similar to that of more resistant mouse strains $(23,24)$. Similarly, high does MCMV infection (in excess of two-logs) in more resistant strains was sufficient to recapitulate DC loss seen in susceptible animals infected with moderate doses of MCMV (Nash and Brown, unpublished data). While these studies support a dominant influence of viral control on DC retention, they also highlight robust differences between the mechanisms of NK cell viral control in different mouse strains. Given that cell-to-cell contacts are required for bi-directional NK-DC interactions and that these cells traffic to similar regions of the spleen during MCMV infection, it is plausible that diverse NK responses to infection engage in unique interactions with DCs. Combined with holistic influences on the host immune environment, these NK-DC interactions could promote distinct DC profiles.

\section{CD8+ $T$ cell immunity}


NK cells and CD8 $+\mathrm{T}$ cells are both armed with cytotoxic granules (perforin, granzyme), death ligands (TRAIL, FasL), and inflammatory cytokines (IFN $\gamma, \mathrm{TNF} \alpha$ ), yet they perform distinct functions as innate and adaptive components of the immune system (112). As discussed above, the kinetics of activation and execution of effector activities for NK and CD8+ T cells temporally separates these cell subsets. This is largely attributed to the differences in how these effector lymphocytes generate diverse specificities for target cells. While NK cells rely on variegated expression of multiple polymorphic, germline encoded receptors to rapidly distinguish healthy and stressed cells, CD8+ T cells utilize somatically re-arranged receptors (V(D)J recombination) that are exquisitely specific to peptide-MHC complexes on the surface of cells. This random rearrangement of receptors theoretically comprises up to $\sim 10^{15}$ unique $\mathrm{T}$ cell receptors (TCR) - though most of these clones are likely deleted during thymic selection (113115). However, specificity comes at a cost, such that in order to sustain this degree of clonal diversity, the frequency of naïve antigen-specific $\mathrm{T}$ cells is significantly lower than the frequency of NK cell subsets with a given receptor display. Thus, small clonal populations of $\mathrm{T}$ cells must be screened, selected, and amplified prior to mediating an effective immune response, and this process of $\mathrm{T}$ cell activation requires time $(\sim 5-7 \mathrm{~d})$ (116). Nonetheless, the advantages to clonal specificity are clear, as this amplification of antigen-specific 'foot-soldiers' contributes to the rapid clearance of target cells and is often necessary to effectively control viral pathogens (117).

In addition to the differences in how NK cells and CD8+ T cells survey their targets, the manner in which they form effector-target conjugates may further distinguish their specific cytotoxic capacity. Using intravital two-photon microscopy, NK cells have 
been previously shown to form short, dynamic contacts with target cells ( $<10 \mathrm{~min}$.), whereas CD8+ effector T cells establish more stable conjugates (>20 min.) (118-120). Despite this difference in effector cell mobility, NK cells have not been demonstrated to be any less cytotoxic than T cells (118). Thus it was hypothesized that NK cells form ephemeral contacts with their targets, and through repeated on-off contact, or through multiple hits from different NK cells, targets can be eliminated with a cumulative delivery of cytotoxic granules. In contrast, it is believed that the tight synapse formation of CD8 T cells with their target cells sustains repeated delivery of perforin and granzyme for effective cell killing (121). Prolonged contact between CD8+ T cells and their targets has been demonstrated to polarize lytic granules towards surrounding cells independent of antigen-restriction, which may account for the observed rapidity of CD8+ T cell killing (122). Recent studies have challenged this paradigm for effector conjugate formation and estimate that $\mathrm{T}$ cell killing of infected cells is actually much slower than previously estimated. Tracking primed cytotoxic T lymphocyte killing of MCMV-infected cells in vivo, Halle et al. observed that individual T cells only killed an average of about 2-16 targets per day and often required multiple $\mathrm{T}$ cell contacts to effectively eliminate infected cells (123). In that study, T cell killing capacity resembled that of NK cells, and suggested that higher densities of antigen-specific $\mathrm{T}$ cells may be required to effectively control viral infection. These models of $\mathrm{T}$ cell killing are not mutually exclusive, since differences in tumor targets and viral infections are likely to affect the manner and efficiency through which $\mathrm{T}$ cells eliminate target cells. These distinct effector-target interactions highlight unique functions for both $\mathrm{NK}$ and CD8 $+\mathrm{T}$ cells, and may even suggest cooperative roles in controlling certain infections or tumors. 


\section{CD8+ $T$ cell differentiation}

One of the most prominent features that has distinguished CD8+ T cells from NK cells is the formation of memory $(116,124)$. While this distinction has been challenged recently with descriptions of persistent, adaptive-like NK cells after exposure to certain viral infections and pro-inflammatory environments $(43,44,125,126)$, many features of memory, including functional heterogeneity and multipotency, continue to distinguish $\mathrm{NK}$ and CD8+ T cells. In response to viral infection clonal CD8+ T cells undergo robust proliferation and traffic to peripheral tissues to eliminate pathogen-infected cells. Upon clearance or effective control of virus, $90-95 \%$ of these cells die during $\mathrm{T}$ cell contraction, and $5-10 \%$ of remaining antigen-specific cells establish the stable, long-lived memory $\mathrm{T}$ cell compartment that exceeds the initial frequency of naïve antigen-specific $\mathrm{T}$ cells. CD8+ memory $\mathrm{T}$ cells provide enhanced host-protection against secondary or reactivated infection through rapid recall response. These responses are aided by a combination of (i) improved ability to proliferate and differentiate into effector T cells, and (ii) the greater frequency of starting antigen-specific T cells (127). Memory $\mathrm{T}$ cells also retain the ability to homeostatically proliferate in the absence of cognate antigen (128), and do so in an IL-7 or IL-15-dependent manner $(129,130)$.

It is important to note that the formation of memory CD8+ T cells is not a fixed proportion of all activated antigen-specific T cells (131), nor does it create a homogenous population of memory cells with equal functions (132). CD8+ T cells integrate a variety of signals, including TCR interactions with cognate peptide-MHC (signal 1), costimulatory interactions with DCs (signal 2), and inflammatory or anti-inflammatory 
cytokines in the host-environment (signal 3), all of which inform their differentiation fate (131). These signals are likely to be influenced by early innate immune responses to pathogen challenge $(8,9)$. Several models for how memory $\mathrm{T}$ cell differentiation occurs have been previously proposed, including (i) the uniform potential model, where activated CD8 $+\mathrm{T}$ cells comprise a homogenous pool of effector cells with equal potential to form memory cells; (ii) the fixed lineage model, which postulates that effector and memory lineage commitment occurs early during initial CD8+ T cell responses to stimulation; and (iii) the progressive differentiation model, where memory precursor cells are established after initial $\mathrm{T}$ cell stimulation, but retain plasticity to differentiate into either terminal effector cells or memory cells (reviewed in (131)). Data for each of these models has been compelling, and its likely that the particular set of immunizing or infectious conditions driving $\mathrm{T}$ cell differentiation can manifest properties of each. What's more is that each of these models highlights a vast heterogeneity within the T cell compartment, and this warrants brief review and consideration.

At the peak of CD8 $+\mathrm{T}$ cell responses to acute viral infection, antigen-specific $\mathrm{T}$ cells can often comprise two broad cell types: short-lived effector cells (SLECs) and memory precursor effector cells (MPECs). SLECs are classic CD8+ effector T cells that are functionally characterized by their cytolytic capabilities (killing assays, granzyme B) and robust production of inflammatory cytokines (IFN $\gamma, \mathrm{TNF} \alpha)$ in response to TCR stimulation. Phenotypically, these cells are CD44 ${ }^{\text {hi }}$, KLRG1+, and CD127- (IL-7R $\alpha$ ) $(127,133-135)$. SLECs are regarded as terminally differentiated cells that die off in the absence of antigen or IL-15, although KLRG1 ${ }^{\text {10 }}$ 'intermediate SLECs' capable of producing IL-2 have been described to retain a limited degree of effector plasticity after 
infection (135). MPECs, which are not yet fully matured memory cells, generally survive the effector-to-memory transition better than SLECs during the course of infection. These cells are phenotypically characterized as CD44 ${ }^{\text {hi }}$, KLRG1-, and CD127+ and functionally known to produce IFN $\gamma, \mathrm{TNF} \alpha$, and IL-2, but not granzyme B $(127,133,134,136,137)$. Additional expression or downregulation of specific co-stimulatory receptors (CD27, CD28) can also help to identify SLECs and MPECs $(138,139)$.

Analysis of transcriptional regulators has helped discriminate between these two effector populations. Two of the most prominent transcription factors include the homologous T-box transcription factors T-bet and Eomesodermin (Eomes), which generally correlate with SLEC and MPEC differentiation, respectively $(140,141)$. While these transcription factor signatures correspond with specific differentiated cell populations, in early effector activation, they function cooperatively to engender full cytotoxic activities and stabilize expression of CD122 (IL2/IL-15R $\beta$ ) (142). Hence, these transcription factors are cooperative, and affect differentiation through a balance of signals. Joshi et al. demonstrated that the development of SLECs was skewed by the level of inflammation experienced by CD8+ effector T cells during infection (IL-12 in particular), and IL-12 signaling increased T-bet expression in a dose dependent manner (133). In contrast, Eomes expression is highly correlated with CD8+ memory T cell differentiation, and in its absence, memory CD8+ T cells are less well preserved and fail to efficiently re-expand after secondary viral challenge $(141,143)$. Studies of additional transcriptional regulators (Blimp-1, Id2, Id3, FOXO1, Bcl-6, TCF7) have continued to inform our understanding of underlying mechanisms of T cell differentiation $(140,141)$. As we have revealed the contributions of various transcription factors, we have also been 
able to explore cell extrinsic signals that regulate the regulators, such as co-stimulatory molecules and cytokines.

Although T cells can be programmed to clonally expand and differentiate into functional effectors after a single brief exposure to antigen (144), during viral infections, they will typically be guided by a variety of inputs (signals 1-3) for variable durations depending on the efficiency of host immunity (116). Several investigations have examined the contribution of co-stimulatory interactions and cytokine signaling to priming CD8+ T cell immunity, most of which have utilized targeted knockouts or monospecific antibodies to investigate the contribution of individual pathways (reviewed in (145-147)). However, we are only now beginning to unpack the importance of how different combinations of co-stimulatory ligands/receptors paired with specific cytokines further influence the qualities of $\mathrm{T}$ cell differentiation. In the context of viral infection, the TNF superfamily (CD27-CD70, OX40-OX40L, 4-1BB-4-1BBL, HVEM-LIGHT) is of particular interest, as many of the receptor-ligand pairs are tightly regulated and have integral roles in influencing T cell proliferation, survival, and differentiation (148). Many of these receptors have demonstrated context dependent impacts on $\mathrm{T}$ cell immunity, such that manipulation of specific TNF superfamily pathways can yield opposing outcomes depending on the dose or type of infection and the timing of intervention (149-154). In addition to the effects of co-stimulatory signals on T cell intrinsic functions, they can also manipulate how $\mathrm{T}$ cells receive, integrate, or deploy extrinsic cytokine signals, which can bear heavily on differentiation of effector $\mathrm{T}$ cells, as previously discussed (155-157). Thus many factors align during viral infection to inform 
effector cell differentiation, and minor alterations in virus control or $\mathrm{T}$ cell priming can provoke substantial impacts.

Beyond the first divergence of T cells into either SLECs or MPECs, MPECs can additionally mature into memory cells with varied features and functions. Sallusto and colleagues first described this divergence in human memory $\mathrm{T}$ cell populations upon noticing differences in chemokine expression (CCR7), localization, and function, and established the concept of central and effector memory subtypes (158). In brief, central memory T cells are characterized by their expression of CD45R0, CCR7 and CD62L (Lselectin), which enable them to traffic to secondary lymphoid organs, extravasate through the high endothelial venules, and home to the T cell zones. In contrast, CD45R0+ effector memory T cells are categorized by their lack of CCR7 and CD62L, and while prevented from trafficking to secondary lymphoid organs, these cells can circulate and penetrate into peripheral tissues and immediately restrain viral infection. The function of these populations demonstrate varied reactivity to antigen stimulation with central memory $\mathrm{T}$ cells exhibiting reduced effector-like functions, but robust proliferation potential and generation of effector cytotoxic T lymphocytes. Effector memory cells, on the other hand, have shorter lifespans and less homeostatic proliferation, but are equipped for more immediate responses to target cells $(132,158,159)$.

The ultimate impact of these two memory cell populations is a diversified memory response to secondary infections. With effector memory cells, antigen-specific T cells are poised for response in greater starting frequency and can mediate immediate protection at the site of infection. At the same time, central memory cells, though slower to react to secondary challenge, maintain the capacity to self-replenish the memory pool 
whilst producing a new pool of effector cells (132). Ideally, both memory populations would be generated in order to benefit from antigen-specific immunity that is both immediate and maintained in reserves, and depending on the infectious challenge, one may be more beneficial than the other $(132,160,161)$. The balance of central and effector memory is likely to be determined by the nature of the pathogen and the antigenic burden. Indeed, pathogens and immunizations that limit antigen exposure tend to favor the formation of central over effector memory $(161,162)$. Conversely, persistent viral infections with recrudescent antigen presentation (e.g. CMV) and heterologous prime/boost vaccinations bias $\mathrm{T}$ cells towards effector memory cells via recurrent peripheral exposure to antigen $(159,163,164)$.

Despite these observations, the progression of signals and primacy of differentiated memory populations is still hotly debated. The linear differentiation model posits that effector memory cells comprise an intermediate subpopulation of memory cells, and in the continued absence of antigen, these cells will revert to central memory (161). Alternatively, the progressive differentiation model argues that effector and central memory cell differentiation is determined by the duration and/or chronicity of antigen/signal exposure, such that sustained or repeated stimulation favors effector memory cell differentiation and that limited or delayed exposure primes central memory (165). Interestingly, in both models, the extent of TCR-antigen stimulation during initial acute infection determines the rate of effector-to-central memory conversion or the ratio of effector to central memory differentiated cells, respectively . Further studies to clarify the host-factors that influence antigen abundance and persistence during acute infection will be invaluable to the design of effective vaccines against a bevy of chronic infections. 


\section{CMV-specific CD8+ $\mathbf{T}$ cell responses}

While NK cells have co-evolved with CMV and have acquired unique receptor repertoires to efficiently detect and eliminate CMV-infected cells $(166,167)$, an adaptive T cell response to CMV is still required to ultimately control active viral replication. Cytotoxic T lymphocytes have been known to provide critical resistance against CMV in both humans and mice for some time. Both specific transfer of primed T cells and interventional treatment with IL-2 to expand and activate T cells has been shown to improve antiviral efficacy $(5,168-171)$. Studies of MCMV in severe combined immunodeficient (SCID) mice have demonstrated that while NK cells can restrain early viral infection, without an adaptive immune response, these mice succumb to persistent infection (3). Moreover, NK depleted mice or mice with inefficient NK responses to MCMV can eventually control infection in a $\mathrm{T}$ cell dependent manner $(3,24,106)$. Antibodies have been demonstrated to restrict dissemination, but are not critical for direct antiviral control (172).

The requirement for different arms of immunity at distinct phases and tissues of the immune response likely dictates the functional contributions of $\mathrm{NK}$ and $\mathrm{T}$ cell responses. $\mathrm{CMV}$ is a prototypical $\beta$-herpesvirus, and like all herpesviruses can establish lifelong infection through achieving viral latency $(173,174)$. CMV also exhibits progressive dissemination throughout the host and tissue-specific tropisms, with the greatest reservoir of latent $\mathrm{CMV}$ infection detected in the salivary gland. Whereas NK cells can restrict early viral dissemination and pathogenesis, CD8+ T cells can effectively terminate acute infection and continue to patrol for reactivated virus in peripheral organs 
$(171,175)$. Although both NK cells and antigen-specific CD8+ T cells can be detected in the salivary gland during latent MCMV infection, neither cell subset exhibits potent cytotoxic control against reactivated virus in the gland, and it is the CD4+ T cells that are critical for immune protection $(109,176-178)$. Recent studies, have clarified the importance of memory CD8+ T cells resident in the salivary gland, noting that while they may be inefficient at protecting against reactivated or systemically introduced infection, which undergo several rounds of selection for viral escape mutants, direct infection of the submandibular gland elicits a protective effector memory T cell response $(179,180)$.

In addition to these diverse host mechanisms for immune surveillance and control of CMV, examination of viral evasion tactics underscores unique immune pressures from both NK cells and CD8+ T cells. Common viral strategies to avoid detection by CD8+ T cells involve the regulation of MHC I expression on the surface of infected cells. However, viruses must strike a balance between limiting MHC I presentation of virusderived peptides and maintaining sufficient MHC I levels to prevent "missing-self" detection $(6,181)$. HCMV utilizes several immunoevasin proteins to selectively decrease MHC I expression on the surface of infected cells (Fig. 1.3). Although seemingly redundant, the varied timing of expression, mechanisms of action, and allelic-specificities of these immunoevasins indicate a tightly orchestrated manipulation of MHC I expression contributes to HCMV's successful evasion of the immune system. Both US3 and US6 non-specifically retain MHC I in the endoplasmic reticulum (182-184). US2 and US11, on the other hand, target and bind to specific, non-overlapping alleles of HLAA and $-\mathrm{B}$ molecules for degradation in the cytosol, leaving residual HLA-C and -E expression to bind NK inhibitory receptors and protect against NK-mediated lysis (185- 
187). The non-overlapping specificities of these latter immunoevasins are indicative of the virus's acquired responses to the high degree of MHC I polymorphism (188). MCMV also encodes several glycoproteins that modify surface expression of MHC I molecules. MCMV gp40 sequesters loaded MHC I molecules in endoplasmic reticulumGolgi intermediate compartments (189), whereas gp48 re-routes mature MHC I molecules to endo-lysosomal compartments for degradation (190).

To balance this broad MHC I downregulation, MCMV gp34 binds to and promotes MHC I expression at the cell surface to protect against missing-self detection $(77,191,192)$. These immunoevasins are both cooperative and antagonistic in function, and through a hierarchy of allele-specific binding, MCMV regulates surface MHC I expression to evade detection by NK cells and CD8+ T cells (193-195). As an additional measure to protect against NK mediated-killing in the absence of MHC I, CMV can also use MHC I-related protein mimics that directly interfere with NK cells. The HCMV glycoprotein UL18, for example, binds to the human inhibitory NK receptor ILT2/LIR-1 to interfere with LIR1+ NK-cell mediated cytotoxicity (196). In MCMV, m144 fulfills an orthologous mechanism of NK-cell inhibition, mimicking key structural features of the H-2 molecule (197-199), although the cognate receptor for this mimic is unknown. m157 is another MHC I-related molecule that may have specifically evolved in MCMV to engage NK-cell inhibitory receptors $(48,49,200)$. Despite its dominant activating properties in $\mathrm{B} 6$ mice and strains bearing $\mathrm{Ly} 49 \mathrm{H}$, it was hypothesized that $\mathrm{m} 157$ initially evolved to inhibit NK cells in the wake of broad downregulations of endogenous MHC I molecules, similar to m144. Support for m157's immune evasive role can be gleaned from studies that found that $\mathrm{m} 157$ variants isolated from wild outbred mice bind an array 
of inhibitory Ly49 with a wide range of affinities while very few of them activate Ly49H (200). Thus, CMV has evolved a number of immunoevasive strategies in order to avoid efficient detection by both NK and CD8+ T cells, which highlights the importance of both of these potent effector cell control mechanisms. 
Figure 1.3. Viral manipulation of the MHC I antigen processing and presentation. The presentation of self- and foreign-peptides on MHC I molecules is integral to the regulation of both $\mathrm{NK}$ and $\mathrm{CD} 8+\mathrm{T}$ cell immunity. Viruses have developed multiple strategies to interfere with MHC I antigen presentation, with the ultimate goal of evading both innate and adaptive immune recognition. Manipulation of MHC I molecules can occur at various stages of the MHC I expression pathway: (1) peptide antagonists produced during proteasomal degradation of cytoplasmic proteins dictate the affinity of MHC I interactions with inhibitory NK receptors and CD8+ TCR; (2) immunoevasins interfere with the proper loading and folding of the MHC I molecules, and even retain properly folded molecules in the endoplasmic reticulum; (3) viral proteins promote the export of MHC I molecules into the cytosol for proteasomal degradation or re-direct MHC I trafficking from the endoplasmic reticulum to endo-lysosomal compartments; (4) viral proteins promote the expression of classical and non-classical MHC molecules, as well as MHC I mimics, to specifically inhibit NK-cell activation. For more information on specific viral immunoevasins not addressed here, please refer to (54). 
Figure 1.3

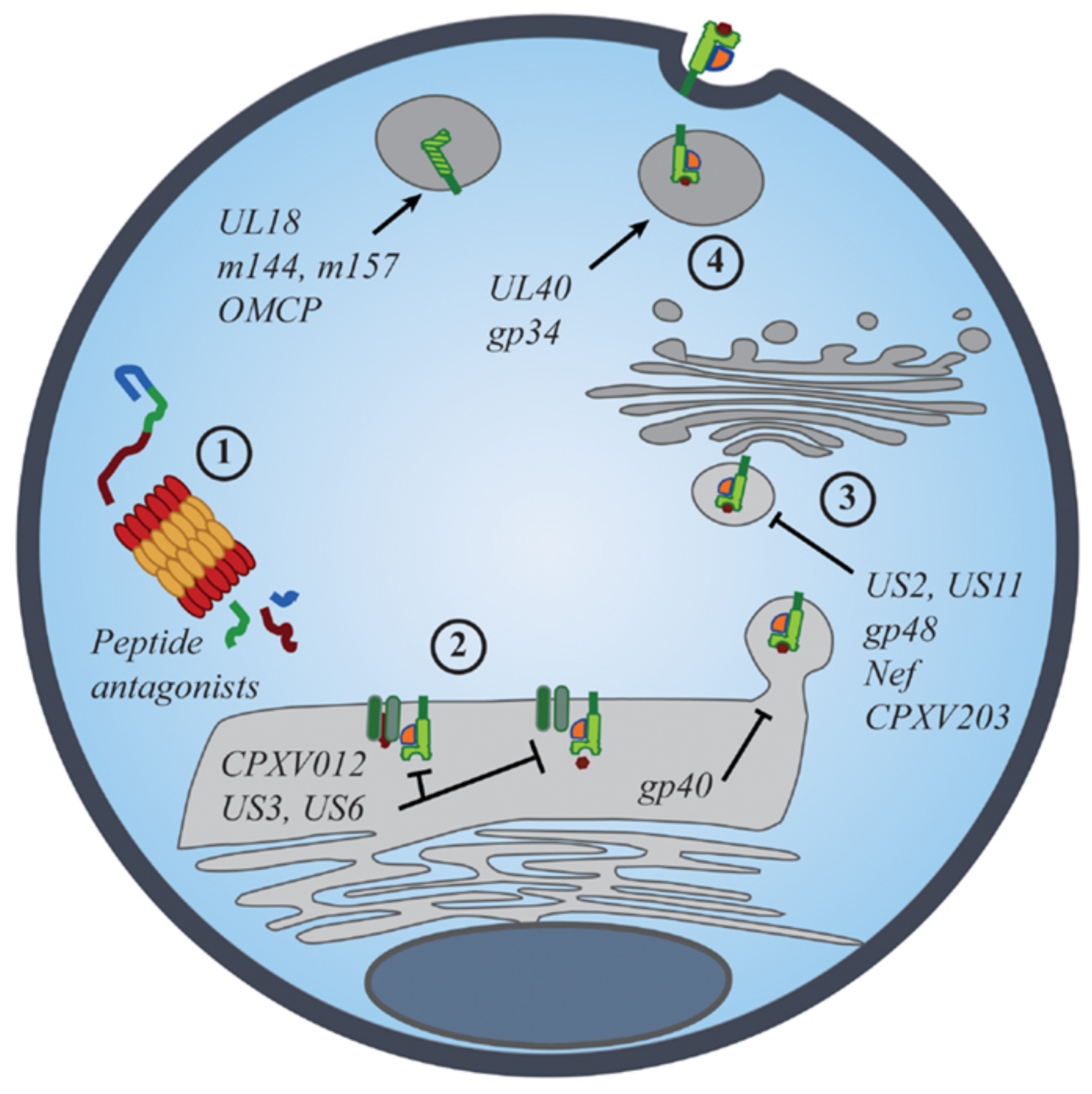


Further support for the importance of $\mathrm{CD} 8+\mathrm{T}$ cell during $\mathrm{CMV}$ infection stems from the hallmark inflation of memory CD8+ T cells seen in CMV infected hosts (164, 201). Memory inflation is characterized by the continuous expansion of CMV-specific memory cells beyond control of acute infection. This is most prevalent in elderly CMV seropositive patients, and up to $15 \%$ of the total $\mathrm{T}$ cell repertoire can be accounted for by CMV-specific CD8+ T cells (164). In humans this was initially thought to occur as a result of persistent or reactivated viral infection (202-204). However, studies in mice have revealed that viral spread and replication is not required to stimulate memory inflation (205-207). Instead sporadic de-silencing of viral genome in latently infected endothelial cells is sufficient to prime CD8+ T cells that circulate throughout the host (208). Interestingly, the clonal repertoire of inflationary memory cells is not restricted to the peptides presented during initial acute infection and can expand to efficiently recognize viral peptides that are exclusively expressed during latent viral infection (209, 210). Thus these cells are not necessarily recently differentiated effector cells from central memory $\mathrm{T}$ cells primed during acute infection, and indeed, are derived from a naïve population of T cells (205). Moreover, the phenotype of these cells resemble that of persistently stimulated cells, and appear to be more terminally differentiated with little homeostatic proliferation $(159,205,211)$. Altogether, the presence of inflationary memory cells helps to boost host immune sensing for reactivated CMV infection, and although energetically demanding, this likely reflects the importance of specifically restraining persistent viral infection.

\section{NK cell influences on $T$ cell immunity}


With NK cells and T cells exhibiting overlapping periods of effector activity during most viral infections, its not surprising that NK cells have recently been described to be important regulators $\mathrm{T}$ cell immunity. Just as the detection of PAMPs and DAMPs can inform the nature of systemic immunity to infection or cancer (8), the manner and quality of early NK cell responses to viral challenge can mold ensuing adaptive immune responses $(11,12)$. Specific activation of NK cells can affect the magnitude and dissemination of early viral replication, which can directly impact the abundance of antigen available for DC cross-presentation to CD8+ T cells $(4,91,93)$. The efficiency of NK cell viral control has also been linked to the preservation of secondary lymphoid organ architecture and maintenance of white pulp for efficient induction of adaptive immune responses $(10,94,212)$. In addition to cytotoxicity, specific cytokine production by NK cells including IFN $\gamma, \mathrm{TNF} \alpha$, GM-CSF, and IL-10 can influence the maturation, trafficking, and co-stimulatory potential of DCs $(20,21,99,100,213,214)$. These cytokine signals can also act on $\mathrm{T}$ cells directly and affect differentiation, particularly IFN $\gamma$ and IL-10 (215-222). Finally, NK cells have been shown to target and eliminate activated $\mathrm{CD} 4+$ and $\mathrm{CD} 8+\mathrm{T}$ cells during certain viral infections, which restricts the development of adaptive immunity and can impact both the persistence of viral infection and the extent of immunopathology mediated by CD8+ T cells $(13-17,223)$. Whether the impacts of NK cells on CD8+ T cells are supportive or suppressive to adaptive immunity likely depends on the host-intrinsic and virus-specific conditions that shape the nature of the NK response.

The coordination of NK and CD8+ T cells responses to MCMV infection has long been recognized as a classic paradigm for the sequential roles of innate and adaptive 
immunity: Early NK cell responses to infection limit the extent of viral spread, and consequently restrict the effects of viral- or cytokine-induced suppression of T cells $(3,4$, 42). Furthermore, the relative efficiency of NK-mediated resistance in genetically distinct mouse strains - with varied expression of NK receptors and MHC I ligands - has been demonstrated to correspond with enhanced $\mathrm{CD} 8+\mathrm{T}$ cell immunity $(23,24)$. In those studies, the predominant influence of efficient NK cell control on T cells was mediated through effective protection or recovery of DCs during MCMV infection. Whether or not NK-DC interactions additionally shaped the maturation and priming potential of these protected populations has yet to be determined, although experimental cessation of viral control via NK-independent means was demonstrated to sufficiently rescue enhanced accumulation of effector T cells. Despite this, coordination between NK and $\mathrm{CD} 8+\mathrm{T}$ cell activities is not always positively correlated. Alternative studies examining the impact of NK depletion and less efficient NK responses to MCMV have reported improved $\mathrm{T}$ cell immunity despite experiencing heightened viral levels in the absence of efficient NK-mediated viral control (106, 109, 224, 225). Interestingly, improved $\mathrm{T}$ cell priming in at least one study was attributed to the protection of infected DCs from NK cell killing, which were shown to efficiently prime T effector cells ex vivo. Thus, even within the context of MCMV, variable impacts of NK cells on DCs and T cells can be seen.

To explain these diverse outcomes mechanistically, Waggoner and colleagues proposed a 'rheostat' model, such that the impact of NK cells on T cell immunity is dependent on the level or dose of viral infection $(13,226)$. In this model, depletion of NK cells during a low dose infection would elevate the infection to moderate titers, but 
not so much that they suppress T cells; in contrast, NK depletion or inefficient NK control under moderate dose conditions could raise the titers significantly and prevent $\mathrm{T}$ cell priming through virus-induced immunosuppression. Therefore, a 'Goldilocks zone' of NK-restricted viral spread must exist in order to prime the T cells 'just right' without invoking suppressive programs. While this inflection point of NK control may be best understood with regard to viral antigen, additional antiviral factors must also be considered, such as the distinct cytokine milieu, lymphoid organization, and the balance with various immune cells like DCs, macrophages, and monocytes after infection. Moreover, intrinsic features of how the NK cell responds to infection will further affect their impact on adaptive immunity, as host-specific NK responses can vary greatly in their functions and phenotypes.

In addition to $\mathrm{T}$ cell support or immunosuppression occurring as a byproduct of NK-mediated viral control, the rheostat model also proposes a potential immunoregulatory role for NK cells to prevent T cell-mediated immunopathology (13). This notion is perhaps best supported by studies of persistent lymphocytic choriomeningitis virus (LCMV; clone 13) infection, which activates NK cells, but does not invoke a virus-specific NK response (3). During LCMV infection, NK cells can regulate adaptive $\mathrm{T}$ cell immunity through direct cytotoxicity of activated APCs, CD4+, and CD8+ T cells $(13,15,18,223)$. A number of mechanisms exist to prevent constitutive NK cytotoxicity of activated immune cells, including type I IFN-mediated upregulation of inhibitory MHC I and downregulation of natural cytotoxicity receptor ligands on T cells, as well as expression of the 2B4 inhibitory signaling lymphocyte activation molecule (SLAM)-receptors on NK cells that can inhibit cytotoxicity 
independent of MHC I $(16,17,223)$. Nevertheless, even with these inhibitory pathways in place, NK cells can still restrict the development of protective $\mathrm{T}$ cell immunity. Interestingly, depending on the dose of infection, this immunoregulation can be hostprotective (13). While NK cells do not control LCMV infection directly, their restrictions on $\mathrm{T}$ cell immunity early after infection can indirectly affect the spread of virus, and consequently the extent of virus-mediated T cell exhaustion. Under high dose LCMV (clone 13) infections, NK restriction of $\mathrm{T}$ cells leads to a persistent, but non-lethal viral infection that overwhelms and exhausts $\mathrm{T}$ cell immunity; this limits immunopathology mediated by cytotoxic CD8+ T cells. On the other hand, with moderate doses of LCMV (clone 13), early NK regulation of $\mathrm{T}$ cells provokes a persistent infection that continuously stimulates $\mathrm{T}$ cells without inducing lymphocyte exhaustion, and prolonged T cell immunopathology can lead to host mortality. Similar observations have also been made for MCMV, whereby the absence of total or virus-specific NK cells during acute infection enhances effector $\mathrm{CD} 8+$ and $\mathrm{CD} 4+\mathrm{T}$ cell activities in the spleen and salivary gland, respectively, but also permits greater $\mathrm{T}$ cell mediated pathology and mortality $(221,227)$. Hence, the measure of $\mathrm{T}$ cell accumulation and effector activity does not alone indicate protective adaptive immunity, and the context in which these responses are elicited can affect the host immensely.

Studies investigating the influence of NK cells on T cell immunity have generally regarded NK cells as a broad class of innate lymphocytes, relying on complete NK depletions to determine their impact under a set of given conditions. However, NK cells comprise an extremely heterogeneous population of lymphocytes, and can be further tuned by specific interactions with host-MHC I $(54,56)$. Therefore, subtle variations in 
the manner that NK cells and specific subsets of NK cells respond to viral infection are likely to have lasting impacts on the quality of CD8+ T cell priming and differentiation outside of the kinetics of viral control. This may include differences in specific trafficking and colocalization with other immune cells; unique maturation profiles that inform cytokine production versus cytotoxicity; and proliferation and frequency of cellto-cell encounters. Distinct receptor repertoires may further dictate not only how NK cells detect infection, but also how they interact with other immune cells. Licensed NK cell responses to viral infection are inherently characterized by expression of inhibitory NK receptors $(54,56,57)$, and inhibitory NK receptors have been demonstrated to limit NK killing to T cells $(16,17,223)$. As similar protective mechanisms have also been described to protect NK cells from NK-mediated 'fratricide' (228), it is likely that these measures of inhibition also help to protect a variety of other cell types. Other models of NK cell responses to viral infection demonstrate that NK cells lacking expression of selfinhibitory receptors can dominate responses to infection and have been shown to be much more proliferative and cytotoxic to infected target cells $(82,83)$. Interestingly, these same highly responsive NK subsets have been shown to restrict $\mathrm{T}$ cell immunity through elimination of infected DCs (109). Whether or not inhibitory receptor interactions can inhibit this immune cell killing remains to be determined. Future studies seeking to clarify the impacts of specific NK subsets in hosts with defined MHC I alleles on adaptive immunity are warranted. 


\section{Significance \& Summary}

The need for coordinated innate and adaptive immune responses to persistent viral infections, such as MCMV, has been well established $(3-5,42)$. However, the mechanisms guiding coordination between these immune cell compartments remain less clear. The efficiency of viral control provided by specific NK cell responses to MCMV can be correlated with improved accumulation of CD8+ effector T cells in response to infection $(23,24,42)$. However, it remains to be clarified whether or not distinct mechanisms of NK cell activation and response additionally influence the quality of $\mathrm{T}$ cell differentiation through manipulation of effects on co-stimulation and cytokine profiles. Moreover, as we seek to modify specific arms of immunity through targeted engineering and specific immune depletions, it is paramount that we understand the span of influence that these cells exert on one another - not only in isolated tissues or in response to specific pathogens, but also in the context of different host environments with varied genetic determinants of immune function.

While several studies have focused on the contribution of Ly49H+ NK cellmediated viral control to adaptive immune development $(23,109,221,224)$, the frequency of this specific receptor in inbred and wild mouse strains is actually quite rare (50). This suggests that alternative mechanisms of NK resistance are likely to exist. Our research group has previously demonstrated that $\mathrm{MHC} \mathrm{I} \mathrm{D}^{\mathrm{k}}$ is a critical resistance molecule for antiviral immunity in mice expressing inhibitory receptor Ly49G2, for which $\mathrm{D}^{\mathrm{k}}$ is a cognate licensing ligand $(71,78)$. Moreover, $\mathrm{D}^{\mathrm{k}}$-licensed $\mathrm{G} 2+\mathrm{NK}$ cells have been shown to accelerate and enhance CD8+ T cell accumulation through specific recovery of DCs (24). Although non- $\mathrm{D}^{\mathrm{k}}$ mice without specific licensed NK responses to 
MCMV can also mount robust CD8+ effector T cell responses, the peak cytotoxic activity of these effectors is delayed. We therefore hypothesized that mice with or without highly specific licensed NK cell responses to MCMV affect T cell priming environments, and that these differences elicit unique CD8 $+\mathrm{T}$ cell differentiation profiles beyond acute infection. The original research described herein addresses two key questions: (i) How do licensed NK cells specifically impact co-stimulatory ligand display by DCs to promote early effector T cell efficient differentiation? (ii) Does early viral control mediated by licensed NK cells influence memory cell differentiation?

In Chapter II, we explore the possibility that $\mathrm{D}^{\mathrm{k}}$-licensed NK cells preferentially induce co-stimulatory ligand expression to efficiently prime CD8+ T cells. We establish that CD8+ T cell responses primed in mice with licensed NK-mediated resistance to MCMV are dependent on CD70-CD27 interactions, and that these CD70 dependent responses can proceed independent of CD4+ $\mathrm{T}$ cell help. However, rather than inducing a preferential up-regulation of CD70 ligand, we observe that licensed NK cell control of MCMV dampens expression of CD70 and CD86. Furthermore, whereas T cells primed in mice with licensed NK cell control of infection are critically dependent on CD70CD27 interactions, primary T cell responses in mice without specific licensed NK cell control can proceed independent of CD70-CD27 co-stimulation. These studies suggest that specific co-stimulatory requirements for priming $\mathrm{CD} 8+\mathrm{T}$ cells during acute infection and the duration of co-stimulatory ligand display can be guided by the efficiency of NKmediated viral control.

In Chapter III, we extend our analyses of effector $\mathrm{T}$ cell priming to examine how licensed NK cells impact early differentiation of CD8+ T cells into SLECs or MPECs. 
We discovered that the presence of specific licensed NK cell responses to MCMV biases early CD8 $+\mathrm{T}$ cell differentiation towards an MPEC fate, whereas mice without specific NK-mediated resistance to MCMV predominantly feature terminally differentiated SLECs. This skewing of virus-specific CD8 $+\mathrm{T}$ cell populations is durable and lasts throughout persistent and latent infection. Both effector and memory $\mathrm{T}$ cells cells from latently infected mice are capable of mediating efficient viral control upon secondary challenge, though to varying degrees depending on the challenge conditions. We believe that the skewed representation of these cell subsets in $\mathrm{D}^{\mathrm{k}}$-disparate mice reflects hostspecific needs for distinct modes of $\mathrm{T}$ cell immunity . 


\section{Chapter II}

Acute virus control mediated by licensed NK cells sets primary CD8+ T cell dependence on CD27 costimulation*

* J Teoh et al. Manuscript accepted. J Immunol (2016). 


\section{Introduction}

NK cells are vital to protect against a variety of viral infections and cancer cells (229). Classical and functional deficiencies in human NK cells are associated with increased susceptibility to recurrent infections, particularly by members of the Herpesviridae family, including cytomegalovirus (CMV) $(36,37)$. While their role as direct antiviral mediators has been well studied $(6,75)$, recent investigations have revealed broader immunoregulatory roles for NK cells, including regulation of adaptive immune responses $(11,12,230)$. As potent cytokine producers and highly cytotoxic effector cells, NK cells can bridge innate and adaptive immunity by aiding dendritic cell (DC) maturation for better $\mathrm{T}$ cell priming $(20,21,97,231)$ and increasing the availability of antigen for cross presentation (93). Efficient NK cell resistance to infection can also limit antigen availability and decrease inflammation, which may decrease the overall magnitude of CD8 $+\mathrm{T}$ cell responses $(106,109)$. Furthermore, NK cell lysis of activated CD4+ and CD8 + T cells, and even other NK cells, which could prevent immune-related pathologies, was also shown to interfere with virus clearance during chronic infections $(13,15-18)$. NK cells therefore serve in a variety of different roles during viral infection, and both the type of viral infection and the host-genetic factors regulating NK cell responses likely guide their involvement in antiviral defenses and host immunity.

MHC I $\mathrm{D}^{\mathrm{k}}$ is a dominant genetic resistance factor that enables NK cells expressing Ly49G2 (G2) inhibitory receptors, for which $\mathrm{D}^{\mathrm{k}}$ is a cognate ligand, to efficiently restrain MCMV infection $(71,78)$. While $\mathrm{D}^{\mathrm{k}}$ expression on healthy cells prevents $\mathrm{G} 2+\mathrm{NK}$ cell responses, altered expression of $\mathrm{D}^{\mathrm{k}}$ during MCMV infection is thought to release G2-mediated inhibition of stimulatory NK receptors, thereby triggering 
efficient target cell lysis (54). Alternately, $\mathrm{D}^{\mathrm{k}}$-dependent licensing could serve to increase G2+ NK cell functionality and specific responsiveness to MCMV targets (54, $59,68,71)$. In either case, licensed G2+ NK cells were found to selectively and specifically expand and limit MCMV spread only in mice with self-MHC $\mathrm{D}^{\mathrm{k}}$ ligands expressed $(68,71,77,78,232)$.

In response to virus infection, NK cells have also been shown to help protect, recover, and license DCs, which resulted in enhancements to cytotoxic T-cell immunity $(23,24,213)$. In particular, we found that $\mathrm{D}^{\mathrm{k}}$-licensed $\mathrm{G} 2+\mathrm{NK}$ cells can accelerate the recovery of $\mathrm{CD} 8 \alpha+$ and $\mathrm{CD} 11 \mathrm{~b}+\mathrm{DCs}$, which then corresponds to enhanced virusspecific CD8+ T cell accumulation (24). Cross-presenting CD8 $\alpha+$ DCs have been shown to be critical for priming non-inflationary MCMV-specific CD8+ T-cell responses (233), and DC interactions with early responding NK cells may indirectly impact the quality of subsequent T-cell priming (97).

Given the differences in CD8+ T-cell kinetics in $\mathrm{D}^{\mathrm{k}}$-disparate mice (24), we predicted that $\mathrm{D}^{\mathrm{k}}$-licensed $\mathrm{G} 2+\mathrm{NK}$ cells regulate co-stimulatory ligands displayed on DC early during MCMV infection. CD28-CD80/86 (234) and TNF superfamily members CD27-CD70 (235), OX40/40L (151), and 4-1BB/4-1BBL (150) were previously shown to provide critical co-stimulatory signals to virus-specific CD8+ T cells, though their spatiotemporal importance varied over the course of MCMV infection (153). Whereas $\mathrm{CD} 28$ is necessary for T-cell expansion during initial priming events $(234,236), \mathrm{OX} 40$ and 4-1BB pathways have been demonstrated to drive latent and inflationary CD8+ Tcell accumulation during persistent MCMV infection $(150,151)$. CD27 plays an interesting role in MCMV infection since it has been shown to be critical for both acute 
non-inflationary and persistent inflationary accumulation of MCMV-specific CD8+ T cells (235). Moreover, immature NK cells express CD27, and several studies have correlated CD27 stimulation with the functional responsiveness of NK cells (237-239). Although these co-stimulatory pathways have been extensively studied, whether NK cells also specifically license DC for co-stimulatory ligand expression is unknown. Moreover, as host- and virus-specific effects influence how CD8+ T cells are primed via specifically licensed DC, understanding the NK cell role in shaping DC co-stimulatory ligand display is highly relevant $(146,149)$.

This study investigated how $\mathrm{D}^{\mathrm{k}}$-licensed G2+ NK cells accelerate and shape virus-specific $\mathrm{CD} 8+\mathrm{T}$ cell accumulation during acute infection. We predicted that licensed NK-mediated virus resistance would enhance DC licensing by regulating costimulatory ligands required in CD8+ T-cell priming. We found that CD70-CD27 costimulatory interactions were required in licensed NK cell enhancement of CD8+ T-cell responses. Although CD70 ligand up-regulation in DCs was found to be a common response to MCMV, irrespective of the extent of virus resistance, we discovered that the efficiency of NK-mediated resistance determined the requirement for specific costimulatory cues to prime CD8 $+\mathrm{T}$ cells. Thus, in mice without licensed NK-mediated MCMV resistance, CD8+ T-cell priming was delayed and occurred independent of CD27 signaling. Our results indicate that specific virus control via licensed NK cells influences the set of cues that prime and shape primary effector CD8+ T-cell responses. 


\section{Results}

\section{CD70 prompts accumulation of antiviral CD8+ $T$ cells without impacting licensed}

\section{NK cell responses to MCMV}

We first examined DC co-stimulatory effects on virus-specific T cells acquired during MCMV infection in MHC I $\mathrm{D}^{\mathrm{k}}$ congenic mice with licensed G2+ NK cells. Heterozygous $\left(\mathrm{D}^{\mathrm{k} / \mathrm{b}}\right)$ mice were used so that MCMV M45-specific (i.e. M45- $\mathrm{D}^{\mathrm{b}}$ restricted) $\mathrm{CD} 8+\mathrm{T}$ cells could be analyzed. As expected, CD3 $+\mathrm{T}$ cells on the whole expanded significantly by d 6 in response to MCMV (Fig. 2.1A). However, CD70 neutralization effectively blocked accumulation of total CD8+ and antigen-specific CD8+ T cell subsets, including M45-specific CD8+ T cells, in comparison to isotype-treated control mice (Fig. 2.1A). It is unlikely this was due to a direct effect on NK cells since virus control in spleen was intact at 3.5 and $6 \mathrm{~d}$ post-infection, and G2+ NK cells accrued and matured normally in CD70-blocked mice (Fig. 2.1B-D, Fig 2.2A). In contrast, sole blockade of either CD86 (Fig. 2.2B) or CD80 (Fig. 2.2C) failed to reduce T-cell accumulation, though in combination they did (Fig. 2.2D). These results are consistent with previous work to indicate that both CD27 and CD28 signals contribute to noninflationary T-cell outcomes $(235,240)$. Our results further demonstrated compensatory mechanisms for CD28 stimulation via CD80 or CD86, whereas CD27 was critically dependent on signals from CD70.

CD70-CD27 and CD80/86-CD28 interactions were previously shown to serve complementary and non-redundant roles during influenza infection (236). Whereas CD28 stimulated CD8+ T cells to begin cycling, CD27 promoted survival and accumulation of activated CD8 $+\mathrm{T}$ cells. Herein we observed that transient CD70- 
blockade had little impact on the frequency of total CD3+ T cells, but significantly reduced the relative proportion of CD8 $+\mathrm{T}$ cells $($ Fig. 2.2E). In contrast, dual blockade of CD80/86 significantly reduced the frequency of total CD3+ T cells, but failed to similarly affect the proportion of CD8+ T cells (Fig. 2.2F). These observations may implicate a similar complimentary co-stimulation cascade, as CD28 stimulation drives total T cell accumulation, and CD27 specifically supports the proportional accumulation of activated CD8+ T cells. Interestingly, TLR agonism alone, such as that induced during viral infection, is sufficient to drive CD80 and CD86 expression on DCs, whereas CD70 typically requires additional co-stimulatory input (e.g. CD40/40L) (241-243). We therefore further investigated CD70-CD27 signaling and its accessibility to licensed NKcell regulation. 
Figure 2.1. CD70 prompts accumulation of antiviral CD8+ $T$ cells without impacting licensed NK cells responding to MCMV. MHC congenic $\mathrm{R} 7\left(\mathrm{D}^{\mathrm{k} / \mathrm{b}}\right)$ mice were either PBS-injected (uninf), or treated with rat IgG (isotype) or mAb FR70 ( $\alpha$-CD70) prior to and concurrent with MCMV infection. MCMV levels (d 6) in spleen tissues were measured and fluorescent mAb-stained splenocytes were analyzed by flow cytometry. (A) Graphs show the means $( \pm \mathrm{SD})$ for the number of viable single cell-gated CD3+ CD19- T cells, and total and M45-specific CD8+ T cells for the indicated groups of mice ( $n=3-4$ mice/group). (B) The graph shows individual spleen virus levels in the indicated mice. (C) The bar graphs show the mean $( \pm \mathrm{SD})$ number of total viable single-cell gated CD3- CD19- NKp46+ NK cells (left) and G2+ NK cells (right). (D) Graphs show the mean $( \pm \mathrm{SD})$ number of immature CD27+ CD11b- (left), maturing CD27+ CD11b+ (center), and mature CD27- CD11b+ (right) NK cells. Results are representative of at least 4 independent studies. Statistical comparisons were calculated using one-way ANOVA when appropriate $(* \mathrm{P}<.05, * * \mathrm{P}<.01, * * * \mathrm{P}<.001, * * * * \mathrm{P}<.0001)$. 
Figure 2.1

A

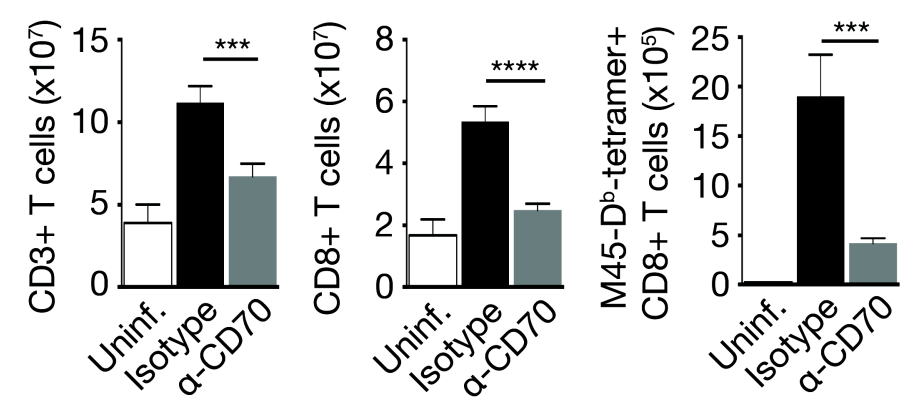

B

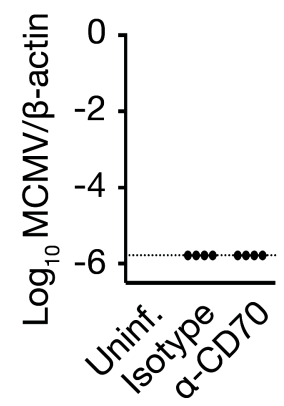

C
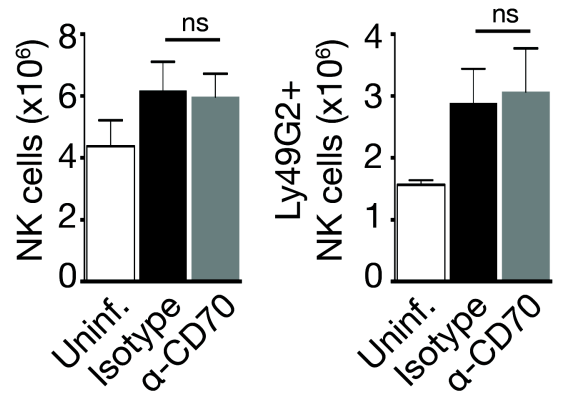

D

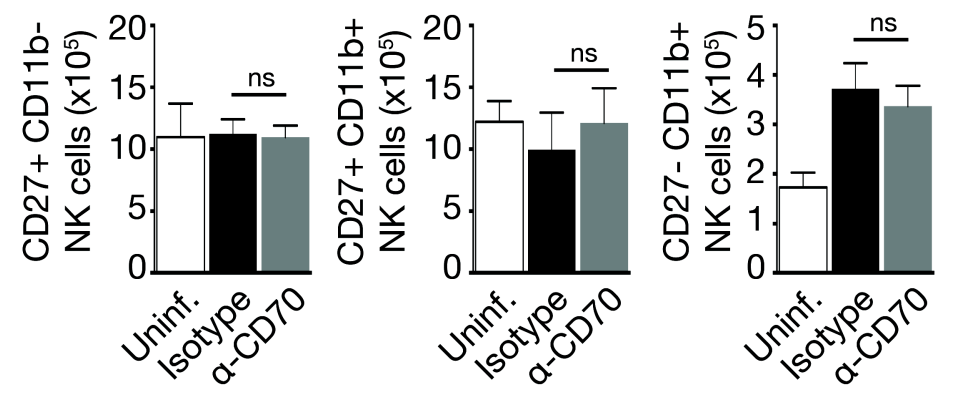


Figure 2.2. Blockade of CD70 specifically impacts the proportion of CD8+ $\mathrm{T}$ cells. MHC congenic R7 $\left(\mathrm{D}^{\mathrm{k} / \mathrm{b}}\right)$ mice were treated with rat IgG (isotype), mAb FR70 ( $\left.\alpha-\mathrm{CD} 70\right)$ (A), mAb GL1 ( $\alpha$-CD86) (B), mAb 16-10A1 ( $\alpha$-CD80) (C), or combination $\alpha-C D 80 / \alpha-$ CD86 (D) during MCMV infection. (A) Individual spleen virus levels are shown for the indicated cohorts (d 3.5). (B-D) Representative bar graphs show the mean $( \pm S D)$ number of total splenic CD3 $+\mathrm{T}$ cells, total CD8+ T cells, and M45-tetramer+ CD8+ T cells per group (d 6). Individual spleen viral levels for each of the cohorts are also shown (far right). (E-F) Graphs show the mean $( \pm \mathrm{SD})$ frequency of CD3+ (left) and CD8+ T cells (right) for $\alpha-C D 70$ (E) and $\alpha-C D 80 / 86$ treatment groups (F). Data are representative of one $(\mathrm{A}, \mathrm{C}, \mathrm{D}, \mathrm{F})$ or two $(\mathrm{B}, \mathrm{E})$ independent experiments $(\mathrm{n}=3-4$ mice/ group). Statistics were calculated using one-way ANOVA where appropriate $(* \mathrm{P}<.05$, $* * \mathrm{P}<.01, * * * \mathrm{P}<.001, * * * * \mathrm{P}<.0001)$ 
Figure 2.2

A

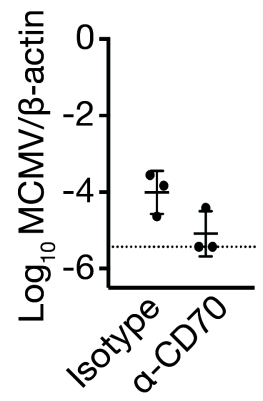

B
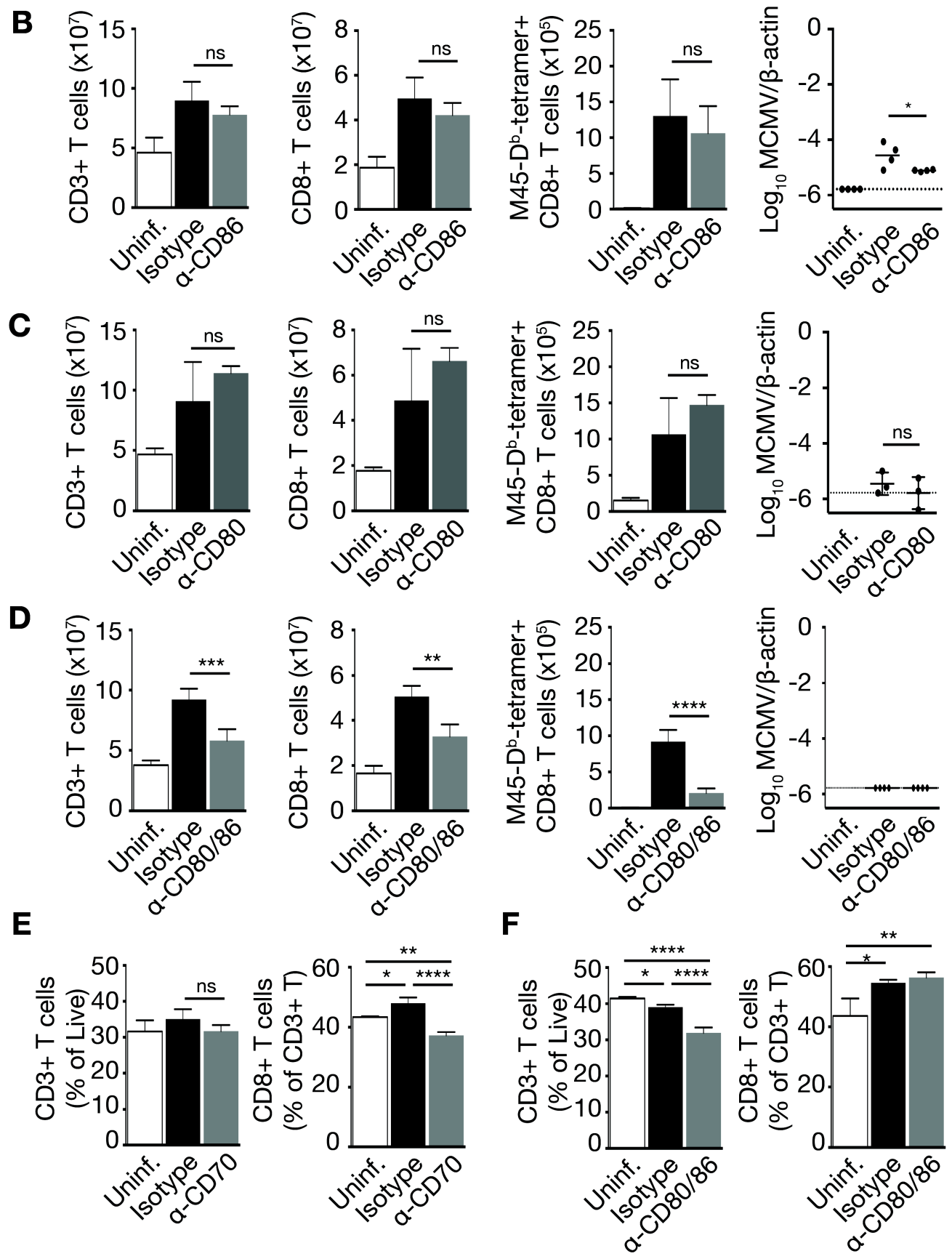


\section{CD70 controls effector CD8+ $T$ cell differentiation during acute infection in mice with licensed NK cell control of MCMV}

During viral infection, CD8+ T cells are exposed to many antigenic, costimulatory and cytokine signals that can affect expression of key transcription factors such as T-box expressed in T cells (Tbet) and eomesodermin (Eomes), and ultimately cell survival and effector functionality $(133,244,245)$. While the duration of antigenic stimulation and exposure to specific cytokine milieus can affect differentiation of CD8+ T effector cells $(133,135,244)$, CD70-CD27 co-stimulation can also impact the quality of effector and memory $\mathrm{T}$ cell differentiation via the regulation of Eomes $(155,156,235$, 246).

To determine its role in T-cell priming, we first examined how CD70 blockade affects CD8+ T-cell differentiation. We assessed CD44 and the $\beta 2$-integrin CD11c to broadly examine total antigen-experienced T cells, rather than solely focusing on M45specific CD8+ T cells $(247,248)$. Importantly, CD44+ CD11c+ T cells were specifically detected after infection, but not in naïve mice (Fig. 2.3A). As with M45-specific T cells, accumulation of CD44+CD11c + CD8 + T cells in response to MCMV was CD70 dependent (Fig. 2.3A-C). We thus further examined CD70's effect on the differentiation of antigen-experienced CD8+ T cells into either short-lived effector cells (SLEC; KLRG1+ CD127-) or memory precursor effector cells (MPEC; CD127+ KLRG1-) as defined by Kaech and colleagues for T cells responding to lymphocytic choriomeningitis virus (LCMV) $(133,134)$. Similar to LCMV infection, antigen-experienced T cells were skewed towards KLRG1+ SLECs in MCMV-infected (d 6) mice, with a near 10-fold accrual of SLECs over naïve controls (Fig. 2.3A, 2.3D). CD70 neutralization decreased 
the accumulation of SLECs to less than half of what was observed in control mice. Even within the population of antigen-experienced CD8+ T cells, CD70 blockade reduced the proportion of CD8 $+\mathrm{T}$ cells that were KLRG1+ (Fig. 2.3D). We also observed significant differences in the numbers of CD127+ antigen-experienced MPECs when we compared control and CD70-blocked mice. However, this could have been due to disparities in the total number of antigen-experienced cells since CD127+ cell frequencies were equivalent (Fig. 2.3E). These results suggested that CD70 stimulation promotes MPEC accumulation during acute MCMV infection, consistent with previous reports (235).

To determine whether the differences in SLEC differentiation also affected functionality, we next assessed CD8+ T-cell cytokines produced in response to ex vivo restimulation with two different MCMV peptides, both of which are expressed during acute infection. Similar to its effect on T-cell differentiation, CD70 blockade dramatically decreased the numbers of M45- and m139-specific IFN $\gamma+$ (Fig. 2.3F) and TNF $\alpha+$ (Fig. 2.3G) CD8 $+\mathrm{T}$ cells in MCMV-infected mice. Even without additional peptide restimulation, $\mathrm{CD} 8+\mathrm{T}$ cells from isotype-treated mice exhibited a trend toward higher cytokine production. Taken together, these results suggested that CD70-CD27 signals prime $\mathrm{CD} 8+\mathrm{T}$ cells to efficiently produce effector cytokines in mice with licensed NK cell control of MCMV. 
Figure 2.3. CD70 controls effector $\mathrm{CD8}+\mathrm{T}$ cell differentiation during acute infection in mice with licensed NK cell control of MCMV. Mice were treated with rat isotype IgG or anti-CD70 and infected with MCMV as in Figure 1. (A) Shown are representative contour flow plots for the frequencies of antigen-experienced (CD44+ CD11c+) T cells (top) and SLEC (KLRG1+ CD127-) and MPEC (CD127+ KLRG1-) differentiated CD8+ T cells (bottom). (B-C) Graphs represent the mean $( \pm \mathrm{SD})$ number of total CD8+ $(B)$ and antigen experienced CD8+ T cells (C). (D-E) Graphs show the mean $( \pm \mathrm{SD})$ numbers and frequencies of antigen experienced SLEC (D) and MPEC (E) CD8+ T cell populations. (F-G) Bar graphs show the intracellular expression of INF $\gamma(F)$ and TNF $\alpha$ (G) by CD8+ T cells for each peptide restimulation. $(n=3-4$ mice/group). Data is representative of at least 4 independent studies. Statistical comparisons were calculated using one-way ANOVA when appropriate $(* \mathrm{P}<.05, * * \mathrm{P}<.01, * * * \mathrm{P}<.001, * * * * \mathrm{P}<$ $.0001)$. 
Figure 2.3

A
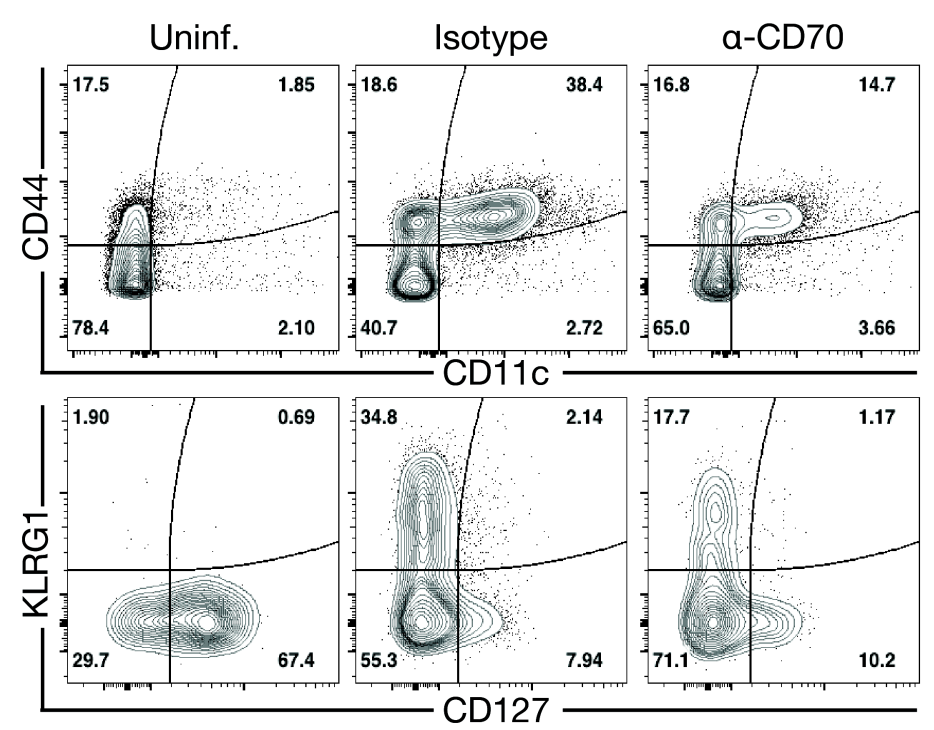

B

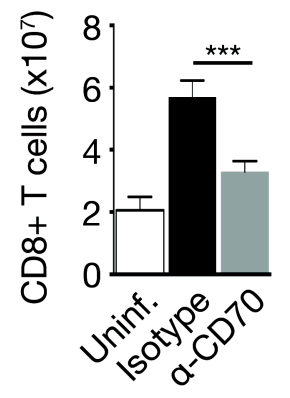

C

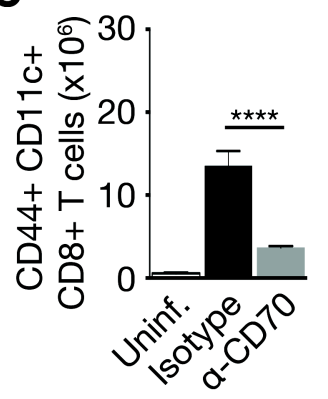

D

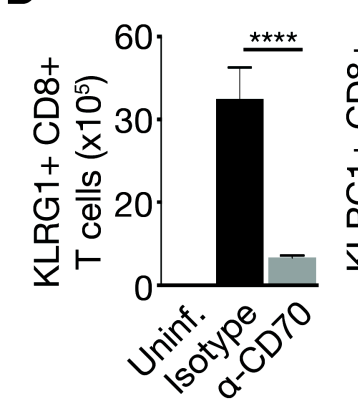

F

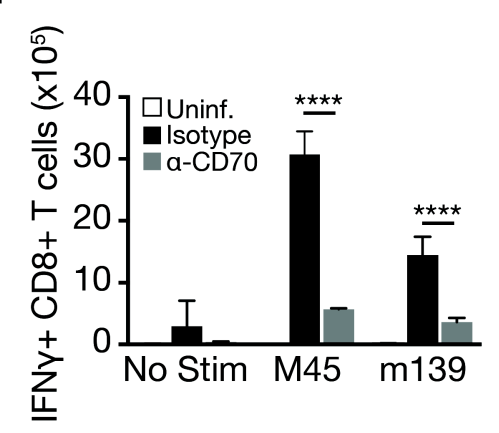

E

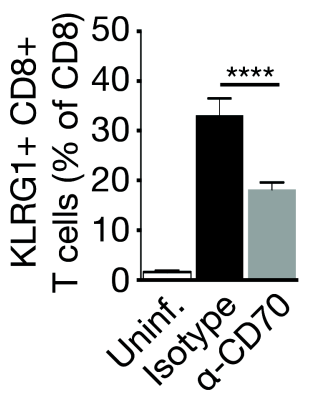

G
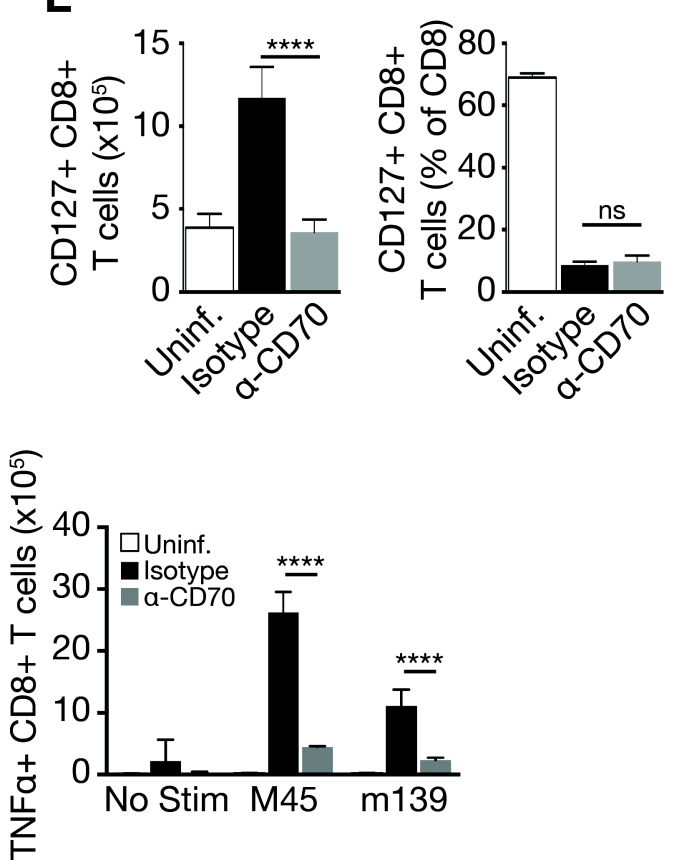


\section{CD8+ $T$ cell dependence on CD70 signaling is intact in CD4+ $T$ cell-depleted mice with licensed NK control of MCMV}

We previously found that licensed NK cells can augment virus-specific CD8+ Tcell immunity without CD4+ T-cell help (24), consistent with prior work that demonstrated CD4+ T cell-independent CD8+ T-cell responses during MCMV infection (176). However, DC licensing for CD70 expression was thought to require co-stimulatory support from CD4+ T cells or helper subsets (e.g. NKT) that support CD40/40L interactions (241-243, 249-252). To pursue this, we analyzed the effect of CD70 neutralization on CD8 + T cells responding to MCMV in CD4+ T cell depleted mice (Fig. 2.4A, 2.4B). The mere absence of CD4+ T cells had little impact on CD $8+T$ cells responding to MCMV, whereas additional blockade of CD70 reduced total CD8+ T-cell numbers somewhat (Fig. 2.4C). We suppose that increased homeostatic T cell expansion after CD4+ T cell depletion may have accounted for the reduced efficacy of CD70 blockade on overall CD8+ T cells (see Figs. 2.1, 2.3). Nonetheless, CD70 blockade significantly muted M45-specific CD8+ T-cells in CD4 T cell-depleted mice (Fig. 2.4D). Still, we observed some variability in the extent of its effect in CD4+ T cell replete and depleted settings, which resulted in 4-fold or 2-fold declines in virus-specific CD8+ T cells, respectively (Fig. 2.1, 2.4D). The results suggested that licensed NK cell control of MCMV may impart CD70-mediated enhancement of CD8+ T cell immunity without CD4+ T cell help.

We next addressed this question by repeating the CD70 neutralization treatment in experimental groups of mice, with or without CD4+ T cells, that could be directly compared (Fig. 2.5). As expected, CD4+ T cell depletion per se had no effect on CD8+ 
T cells responding to MCMV (Fig. 2.5A, 2.5C, 2.5D). Importantly, direct comparisons showed that CD70 signaling was equally important to expand (4-fold) overall and virusspecific CD8+ T cells, irrespective of CD4+ T cells. Similar patterns were also detected for M45-specific SLECs and MPECs (Fig. 2.5E, 2.5F). Thus, these results indicated that enhanced accumulation of MCMV-specific CD8+ T cells in the presence of licensed NK cells is CD70-dependent and proceeds even in the absence of CD4+ T cells.

We next sought to determine whether interactions between CD40L and CD40 were necessary to prime efficient virus-specific CD8+ T-cell responses. While helper CD4+ T cells are perhaps best known for CD40L expression, the ligand can also be displayed by other leukocytes including CD8+ T cells, $\gamma \delta-\mathrm{T}$ cells, NK cells, NKT cells, monocytes, basophils, and mast cells (249). In fact, NK cells were found to drive in vitro maturation of monocytes via CD40/40L interactions, which then primed M. tuberculosis antigen-specific CD8+ T cells (253). Thus, we assessed if licensed NK cells express CD40L in response to viral infection. We found only limited intracellular stores of CD40L, however, and there were no differences observed among licensed and unlicensed NK cells (Fig. 2.5G). To determine if CD40L has a role in helping licensed NK cells to prime CD8+ T cells, we gave repeated doses of anti-CD40L blocking $\mathrm{mAb}$ to $\mathrm{D}^{\mathrm{k}}$ mice during infection. CD40L blockade, however, had little impact on total CD8+ T-cell accumulation in comparison to control mice (Fig. 2.4E). Additionally, CD40L blockade yielded only modest and insignificant reductions in M45-specific CD8+ T cells (Fig. 2.4F). In aggregate, these data demonstrated that CD70 effectively costimulates CD8+ T cell immunity in a CD4+ T cell- and CD40/40L-independent manner in mice with licensed NK cell control of MCMV. 
Figure 2.4. CD8+ $\mathrm{T}$ cell dependence on CD70 signaling is intact in CD4+ $\mathrm{T}$ celldepleted mice with licensed NK control of MCMV Anti-CD4 (GK1.5) depleting antibodies were administered to $\mathrm{MHC}$ congenic $\mathrm{R} 7\left(\mathrm{D}^{\mathrm{k} / \mathrm{b}}\right)$ mice to effectively deplete CD4+ T cells. CD4-depleted mice were additionally treated with rat isotype IgG or antiCD70 blocking antibodies during MCMV infection. (A) The dot plots represent frequencies of splenic CD8+ and CD4 $+\mathrm{T}$ cells from the indicated cohorts (d 6). (B-D) Bar graphs show the mean $( \pm \mathrm{SD})$ of total $\mathrm{CD} 4+(\mathrm{B}), \mathrm{CD} 8+(\mathrm{C})$ and tetramer $+\mathrm{CD} 8+\mathrm{T}$ cells (D). Data are representative of 2 experiments with 3-4 mice per group. (E-F) Congenic R7 ( $\left.\mathrm{D}^{\mathrm{k} / \mathrm{b}}\right)$ mice were treated with neutralizing anti-CD40L (MR1) monoclonal antibody during MCMV infection. Bar graphs show the mean $( \pm \mathrm{SD})$ number of total $(\mathrm{E})$ and M45- $\mathrm{D}^{\mathrm{b}}$-tetramer $+\mathrm{CD} 8+\mathrm{T}$ cells at $\mathrm{d} 6$. Data are representative of 3 experiments (n =3-4 mice/group). Statistical analyses were performed using one-way ANOVA $(* \mathrm{P}<$ $.05)$. 
Figure 2.4

A

CD4 T cell depleted

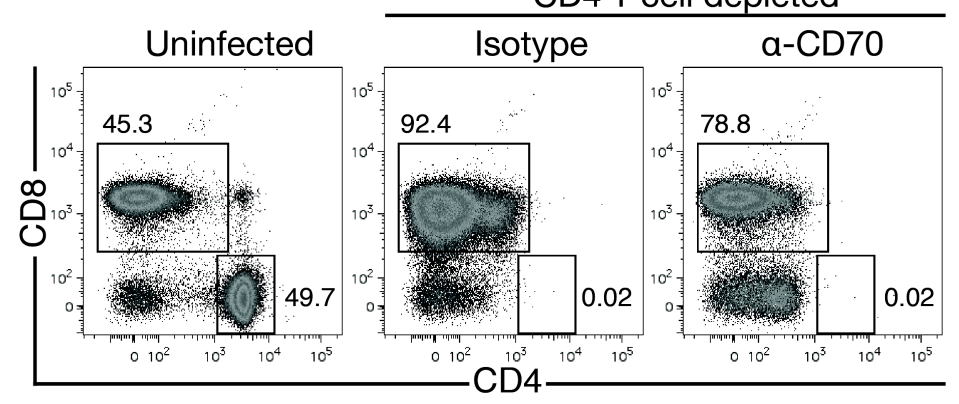

B

C

D
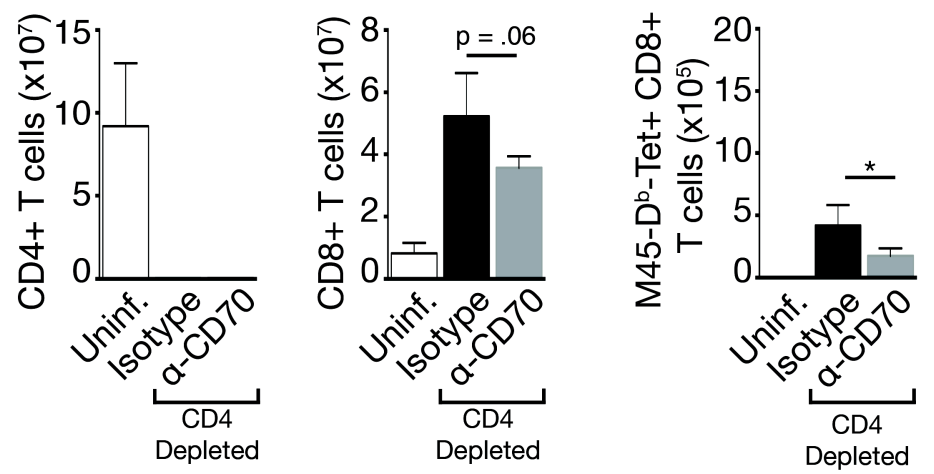

E

F
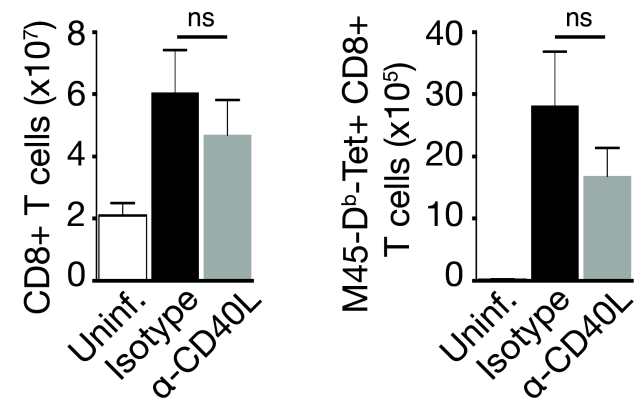
Figure 2.5. CD70-dependent CD8+ $\mathrm{T}$ cell immunity proceeds in the absence of CD4 T cells and licensed G2+ NK cell expression of CD40L. CD4+ T cell replete and depleted $D^{k}$ mice were infected with MCMV and treated with $\alpha-C D 70$ or rat isotype during infection. (A-F) Bar graphs show the mean $( \pm \mathrm{SD})$ number of total splenic $C D 3+$ (A), CD4+ (B), CD8+ (C), and M45-D ${ }^{\mathrm{b}}$-tetramer+ CD8+ T cells (D). (E-F) Mean ( \pm SD) numbers of SLECs (KLRG1+ CD127-) and MPECs (KLRG1- CD127+) were identified within the M45-specific CD8+ T cell population, and are displayed for each of the treatment cohorts. Data are representative of a single independent experiment $(n=4$ mice/group). (G) Mice with $\mathrm{D}^{\mathrm{k}}$ were infected with MCMV, and splenic NK cells were observed for intracellular CD40L expression at $0,36,60$, and $72 \mathrm{~h}$ post-infection. Shown are representative flow plots (left) for total NK-cell expression of CD40L. The histogram (right) shows the mean $( \pm \mathrm{SD})$ number of splenic $\mathrm{CD} 40 \mathrm{~L}+\mathrm{NK}$ cells for $\mathrm{G} 2+$ and $\mathrm{G} 2-\mathrm{NK}$ cells ( $\mathrm{D}^{\mathrm{k}}$-licensed and unlicensed, respectively). Data are representative of 2 independent experiments ( $\mathrm{n}=4$ mice/group). Statistics were performed using one-way ANOVA (*P $<.05, * * \mathrm{P}<.01, * * * \mathrm{P}<.001)$ 
FIGURE 2.5

A

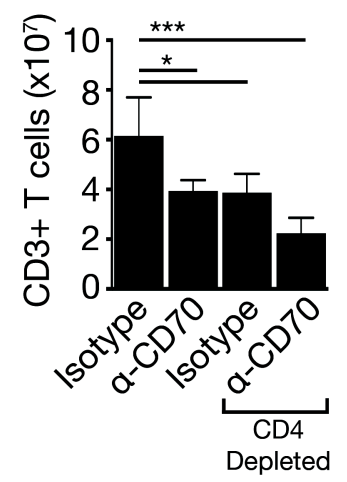

B

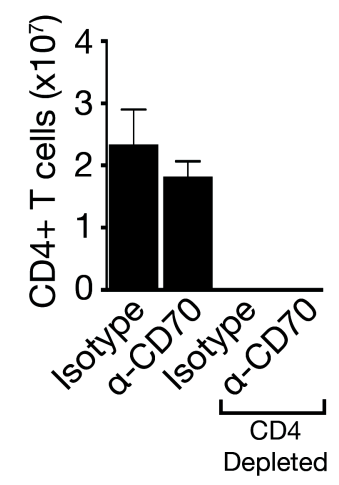

D

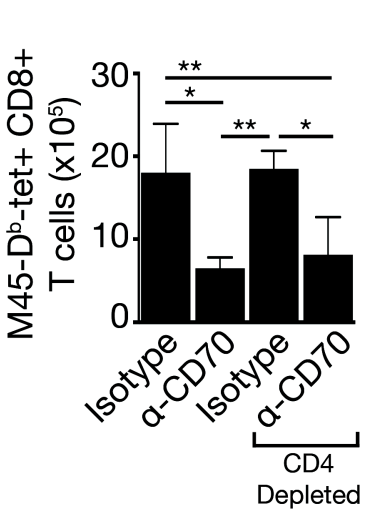

E

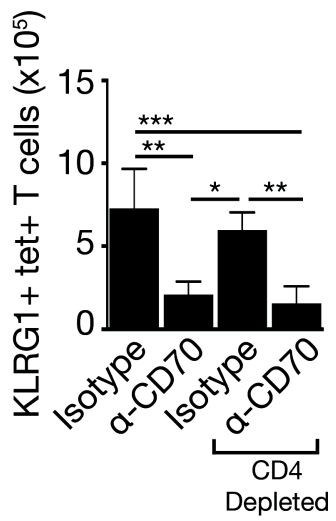

C

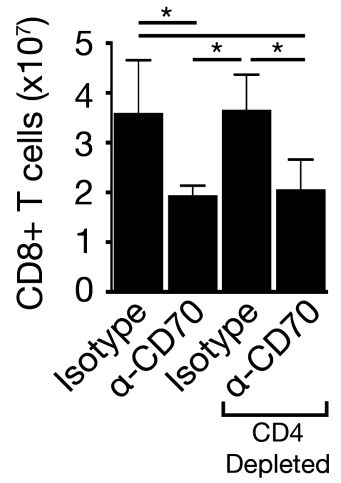

F
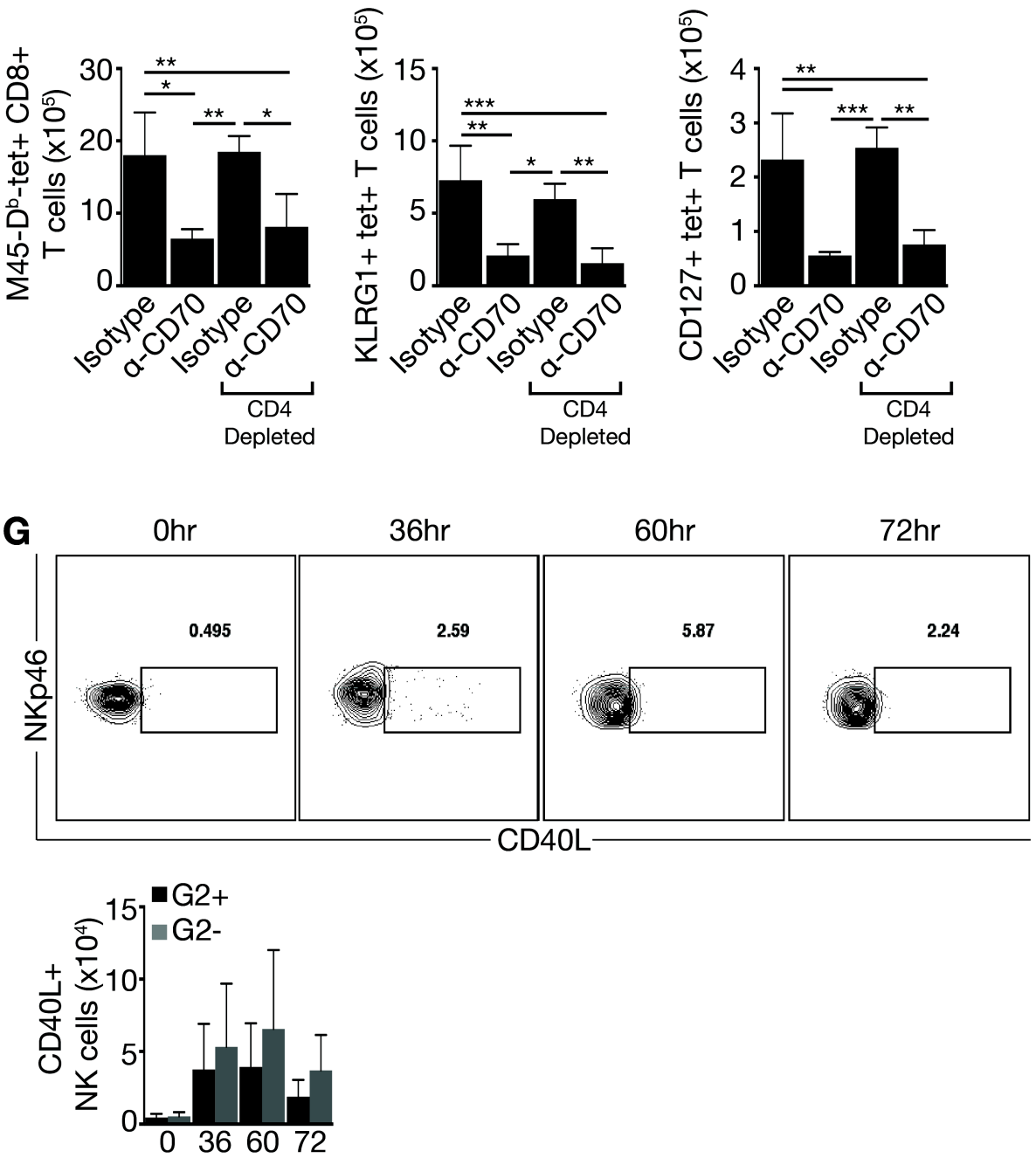

Hours post-infection 


\section{CD27-deficiency dampens virus-specific CD8+ T cell immunity without impairing licensed NK cell control of MCMV}

Next we examined the role of CD70-CD27 co-stimulation in distinct CD8+ T cell immune responses in mice with or without licensed NK cell control of MCMV by assessing the effect of CD27 deficiency in our $\mathrm{D}^{\mathrm{k}}$-disparate mouse strains (see Materials and Methods). To validate the model of licensed G2+ NK-cell virus control in CD27 KO mice, we examined spleen tissues on d 6 after infection. As expected, MCMV was below detection in mice with $\mathrm{D}^{\mathrm{k}}$-licensed NK cells, irrespective of CD27 expression (Fig. 2.6A). In fact, virus spread was fully restrained by 3.5 days p.i., which further indicated that licensed NK-mediated virus control in $\mathrm{D}^{\mathrm{k}}$ mice is not delayed by CD27 deficiency (Fig. 2.7A, 2.7B). In contrast, virus levels were generally 3-logs higher in both strains without $\mathrm{D}^{\mathrm{k}}$. Although larger numbers of total and $\mathrm{G} 2+\mathrm{NK}$ cells responding to MCMV were generally observed in $\mathrm{CD} 27 \mathrm{KO}-\mathrm{D}^{\mathrm{k}}$ mice, they did not differ significantly from CD27 KO NK cells in non- $\mathrm{D}^{\mathrm{k}}$ mice (Fig. 2.7C, 2.7D). Significant elevations in activation $(\mathrm{CD} 69+)$ and maturation $(\mathrm{CD} 11 \mathrm{~b}+)$ markers were also observed in the CD27 KO NK cells, which suggested that these cells were indeed responding and specifically controlling viral infection (Fig. 2.7E, 2.7F). Non- $\mathrm{D}^{\mathrm{k}}$ mice exhibited a 2-fold greater number of total CD69+ NK cells (d 3.5), which likely reflected the sustained elevation of splenic viral levels and the continued non-specific activation of NK cells, as these parameters have been previously correlated (78). Nonetheless, these results indicated that licensed NK cell-mediated resistance to MCMV is CD27-independent.

While the ability of $\mathrm{D}^{\mathrm{k}}$-licensed NK cells to efficiently respond to and restrain acute MCMV infection was unhindered by CD27 deficiency, previous work established 
that $\mathrm{CD} 8+\mathrm{T}$ cell expression of CD27 is necessary to mediate CD70-dependent virusspecific $\mathrm{T}$ cell immunity (235). Thus, we further examined the role of CD70-CD27 signaling in driving distinct $\mathrm{CD} 8+\mathrm{T}$ cell responses to MCMV in our $\mathrm{D}^{\mathrm{k}}$-disparate congenic strains. As expected (254), CD4+ and CD8 $+\mathrm{T}$ cell numbers in naïve mice were unaffected, indicating that $\mathrm{CD} 27$ had little impact on the homeostatic maintenance of naïve T cells (Fig. 2.6B-D). However, MCMV induced accumulation of total and M45specific CD8 $+\mathrm{T}$ cells in mice with both $\mathrm{D}^{\mathrm{k}}$ and $\mathrm{CD} 27$ (Fig. 2.6B, 2.6D, 2.6E). Total numbers of CD4 $+\mathrm{T}$ cells were unaffected by the presence of $\mathrm{D}^{\mathrm{k}}$ or $\mathrm{CD} 27$ (Fig. 2.6C), however previous studies have identified deficiencies in antigen-specific CD4+ $\mathrm{T}$ cell responses to MHC II restricted MCMV peptides in the absence of CD27 (235). As with CD70 neutralization, CD27-deficiency severely limited M45-specific CD8+ T cell accumulation in mice with $\mathrm{D}^{\mathrm{k}}$-licensed $\mathrm{NK}$ cell control of MCMV. In fact, CD8+ T cells expanded similarly sans CD27 signaling regardless of whether $\mathrm{D}^{\mathrm{k}}$ was expressed.

In addition to CD27-dependent accrual of virus-specific CD8+ T-cells, we found that CD27 was also critical for priming SLECs and IFN $\gamma$-competent CD8+ T cells in $\mathrm{D}^{\mathrm{k}}$ mice. In comparison, the absence of either $\mathrm{CD} 27$ or $\mathrm{D}^{\mathrm{k}}$ significantly interfered with CD8+ T SLEC differentiation and cytokine production at d 6 (Fig. 2.6F, 2.6H). Once again, these effects were not limited to a single T-cell epitope, as peptide re-stimulation with $\mathrm{m} 139$ recapitulated the difference in numbers of cytokine producing CD8+ T cells amongst different groups. In the M45-specific population, there was little evidence of CD8+ T MPEC differentiation at d 6 (Fig. 2.6G). In aggregate, these results indicated that although licensed NK cell activation, accumulation and control of MCMV proceeded 
independent of CD27, CD8+ T cell priming in the wake of efficient MCMV control still required CD70-CD27 signaling for early accumulation of KLRG1+ SLECs. 
Figure 2.6. CD27-deficiency dampens virus-specific CD8+ T cell immunity without impairing licensed NK cell control of MCMV CD27 KO, CD27 KO-D ${ }^{\mathrm{k}}, \mathrm{CD} 27 \mathrm{WT}$ and CD27 WT-D ${ }^{k}$ mice were infected with MCMV for $6 \mathrm{~d}$. (A) The graph shows individual spleen virus levels in the indicated mice. (B-E) Bar graphs show the mean $( \pm \mathrm{SD})$ number of total CD3+ (B), CD4+ (C), CD8+ (D), and M45-D ${ }^{\mathrm{b}}$-tetramer+ T cells $(\mathrm{E}) .(\mathrm{F}-$ G) Bar graphs show the mean $( \pm \mathrm{SD})$ number of tetramer+ SLECs $(F)$ and MPECs $(\mathrm{G})$ per spleen at d 6. (H) Graphs represent the mean $( \pm \mathrm{SD})$ number of total IFN $\gamma+\mathrm{CD} 8+\mathrm{T}$ cells detected for each peptide stimulation (No stimulation, M45, and m139). Data are representative of 3 independent experiments $(n=3-4$ mice/group). Statistical comparisons were calculated using one-way ANOVA $(* \mathrm{P}<.05, * * \mathrm{P}<.01, * * * \mathrm{P}<.001$, $* * * * \mathrm{P}<.0001)$. 
Figure 2.6
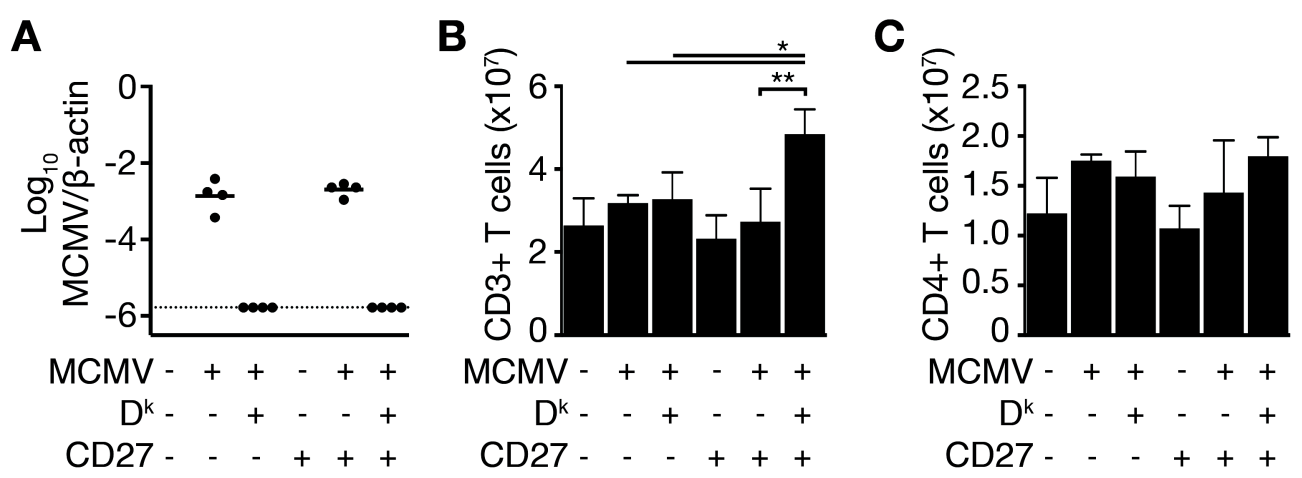

D

E
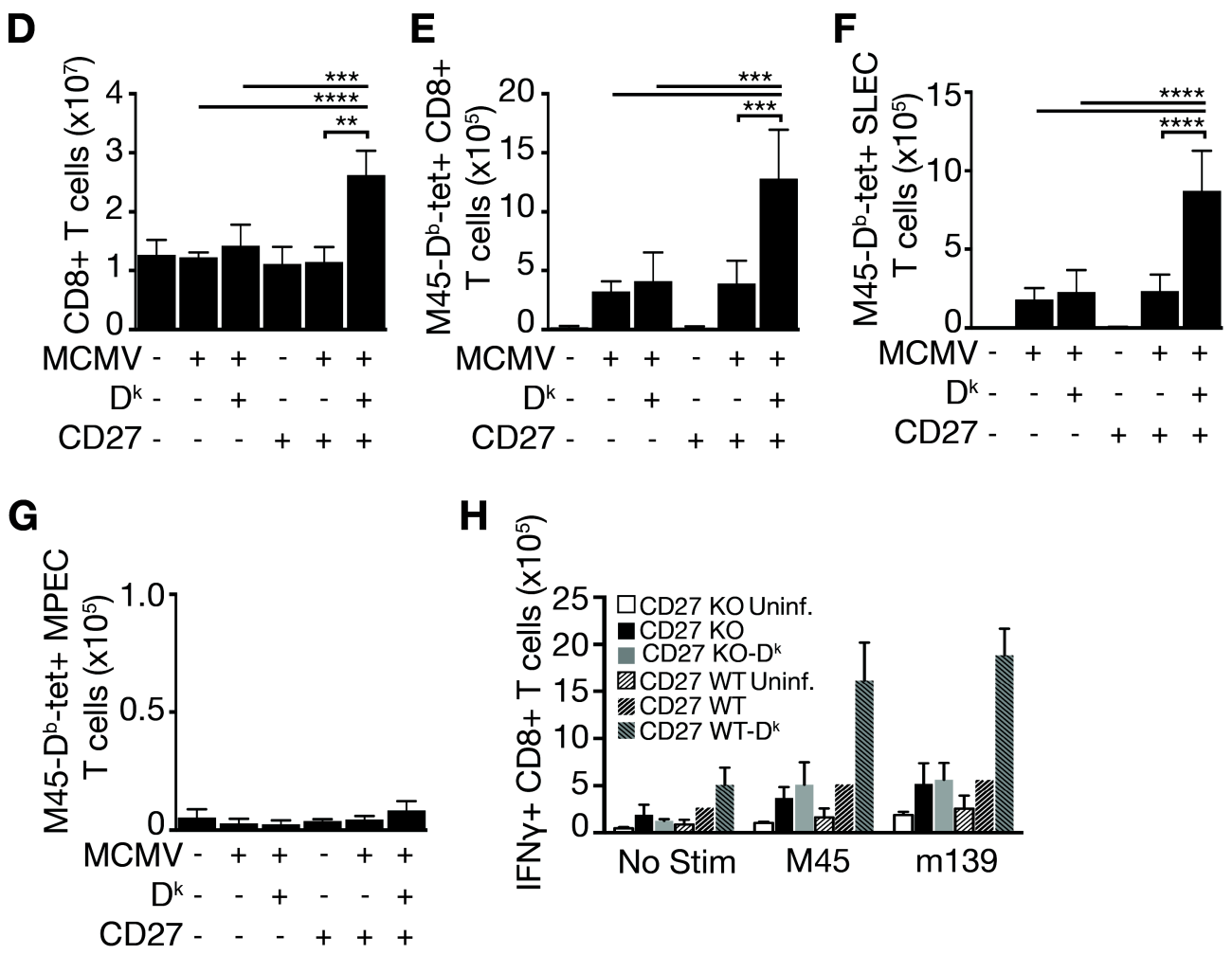
Figure 2.7. CD27 is dispensable for licensed NK cell control of MCMV. D ${ }^{\mathrm{k}}$-disparate CD27 WT and CD27 KO mice were infected with MCMV. (A-B) Scatter plots show individual spleen MCMV levels for CD27 WT (A) and CD27 KO mouse strains (B) at d 3.5. (C-D) Bar graphs show the mean $( \pm S D)$ number of CD3- CD19- DX5+ NK cells $(C)$ and G2+ DX5+ NK cells per spleen (D). (E-F) Bar graphs display the mean $( \pm \mathrm{SD})$ number of activated CD69+ NK cells (E) and mature CD11b+ NK cells (F). Statistical analyses were performed using one-way ANOVA $(* \mathrm{P}<.05, * * \mathrm{P}<.01)$ or unpaired Student $\mathrm{t}$-tests where appropriate $(* * * \mathrm{P}<.001, * * * * \mathrm{P}<.0001)$. 
Figure 2.7
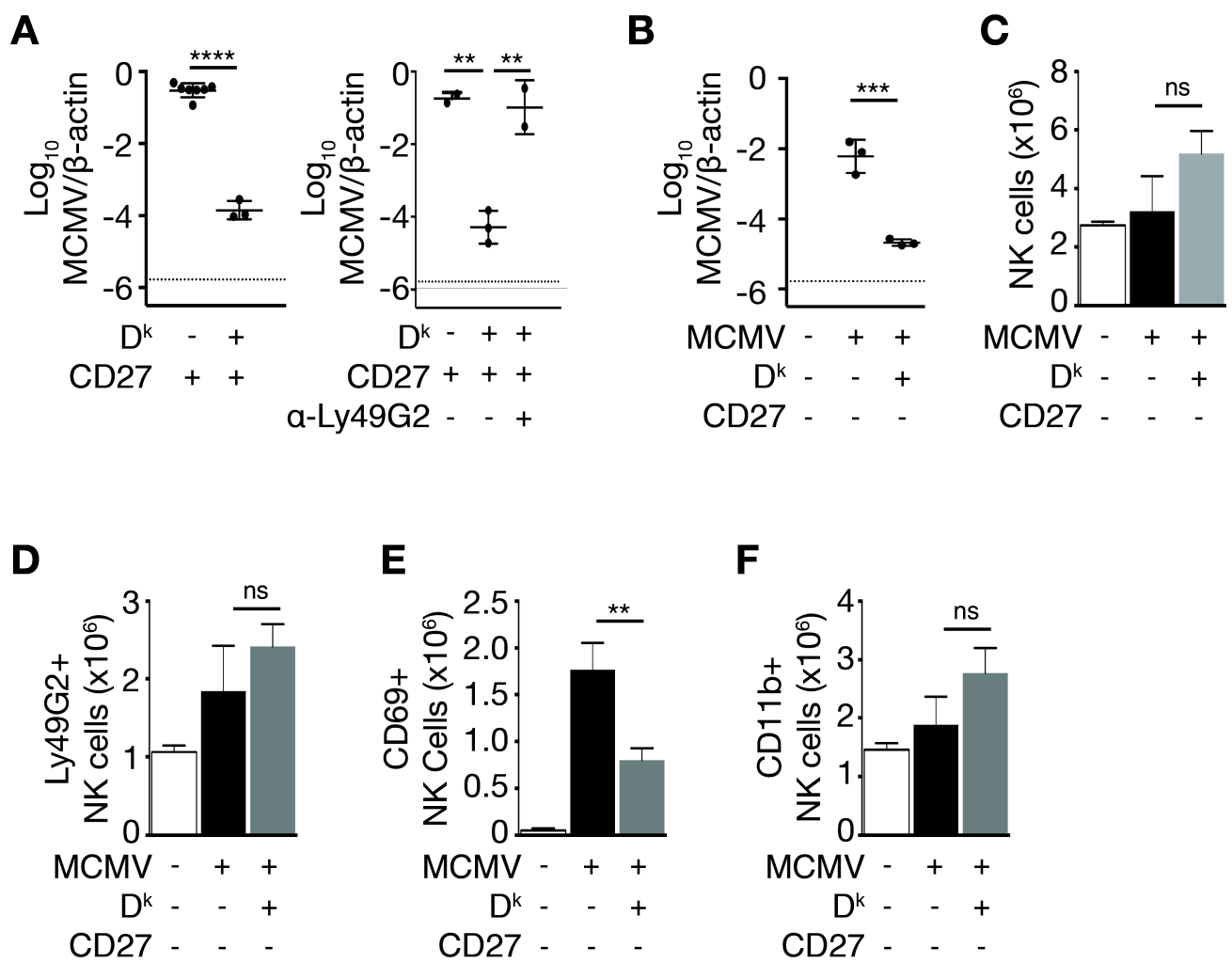


\section{Licensed NK cell control of MCMV adjusts the duration of CD70 expression on DC}

Intriguingly, CD27-deficiency did not further impede CD8+ T-cell accumulation in mice without $\mathrm{D}^{\mathrm{k}}$ (Fig. 2.6D, 2.6E). This suggested one of two possibilities: (i) CD70 could be selectively regulated by efficient NK cell control of MCMV. If so, CD27 expression on CD8+ T cells should have little impact on virus-specific T-cell accumulation in non- $\mathrm{D}^{\mathrm{k}}$ mice. (ii) Alternatively, poor virus control due to the absence of licensed NK cells might interfere with early CD8+ T-cell priming, regardless of access to co-stimulatory mediators. If so, analysis of d $6 \mathrm{~T}$-cell features to test the role of CD70CD27 signaling in non- $\mathrm{D}^{\mathrm{k}}$ mice may be challenging since previous work showed that poor virus control corresponded with a delay in the acquisition of functionally competent effector T cells in vivo (24). Both of these possibilities were therefore pursued to investigate if licensed NK cells regulate CD27-dependent T-cell accumulation.

To investigate whether licensed NK cells influence CD70 expression on DCs, we examined splenic DCs in $\mathrm{D}^{\mathrm{k}}$-disparate mice after infection. MCMV levels were similar at $36 \mathrm{~h}$, but began diverging as early as $48 \mathrm{~h}$ for the two mouse strains (Fig. 2.8A). Surprisingly, $C d 70$ gene expression increased with similar kinetics and magnitude in $\mathrm{D}^{\mathrm{k}}$ and non- $\mathrm{D}^{\mathrm{k}}$ mice early after infection (Fig. 2.8B). While $C d 70$ expression had increased slightly in DC relative to naïve controls by $36 \mathrm{~h}$, it increased substantially in both mouse groups by $48 \mathrm{~h}$ postinfection. By $72 \mathrm{~h}, C d 70$ expression in $\mathrm{D}^{\mathrm{k}}$ mice had returned to baseline, whereas it remained elevated in non- $\mathrm{D}^{\mathrm{k}}$ mice $72-96 \mathrm{~h}$. These data indicated that licensed NK-cell resistance coincided with restrained $C d 70$ expression in DCs. We next examined MCMV-induced DC cell surface expression of costimulatory ligands and MHC II (I-A/I-E) (Fig. 2.8C). Consistent with transcriptional kinetics, both $\mathrm{D}^{\mathrm{k}}$ and non- $\mathrm{D}^{\mathrm{k}}$ 
mice had increased expression of CD70 and CD86 at $48 \mathrm{~h}$ postinfection. For $\mathrm{D}^{\mathrm{k}}$ mice, maximal CD70 and CD86 expression occurred at 48 and $72 \mathrm{~h}$, respectively, and then decreased afterward. In contrast, non- $\mathrm{D}^{\mathrm{k}}$ mice had increased expression (MFI) for both CD86 and CD70 through $96 \mathrm{~h}$ postinfection. These results suggested that co-stimulatory ligands in DCs were negatively regulated in $\mathrm{D}^{\mathrm{k}}$ mice in comparison to prolonged CD70 and CD86 expression in non- $\mathrm{D}^{\mathrm{k}}$ mice. In addition to higher expression of these maturational markers, DC frequencies and total numbers of DCs expressing CD70 and CD86 were also significantly elevated in non- $\mathrm{D}^{\mathrm{k}}$ mice at $96 \mathrm{~h}($ Fig. 2.8D, 2.8E$)$. Whether this was due to downregulation of co-stimulatory ligands in mature DCs, or expansion of immature DCs from pre-DCs in $\mathrm{D}^{\mathrm{k}}$ mice remains to be determined. Thus, counter to expectations, MCMV infection resulted in increased costimulatory ligand gene and protein expression, with slightly greater and extended up-regulation in non- $\mathrm{D}^{\mathrm{k}}$ mice lacking highly efficient licensed NK cell virus control. 
Figure 2.8. Licensed NK cell control of MCMV adjusts the duration of CD70

expression on DC (A) MCMV genome levels in individual $\mathrm{D}^{\mathrm{k}}$-disparate mice at the indicated times postinfection are shown. (B) Spleen CD11c+ DCs were positively selected at the indicated times postinfection. The graph displays the mean $( \pm \mathrm{SD})$ foldchange in normalized $C d 70$ expression relative to naïve DCs. (C) The histogram overlays of viable (single-cell) CD3- CD19- MHC II ${ }^{\text {hi }}$ gated DCs demonstrate representative expression of co-stimulatory ligands CD70 (top) and CD86 (middle), as well as MHC II (I-A/I-E) (bottom) for the indicated time points (black dashed lines $=\mathrm{D}^{\mathrm{k}}$; red solid lines $=$ non- $\mathrm{D}^{\mathrm{k}}$; shaded graphs $=\mathrm{CD} 70 \mathrm{FMO}$ or $\mathrm{d} 0 \mathrm{CD} 86$ and MHCII). Median fluorescent intensities for each of the ligands from $\mathrm{D}^{\mathrm{k}}$ (top black) and non- $\mathrm{D}^{\mathrm{k}}$ (bottom red) mice are also displayed in each histogram. (D,E) Graphs represent the total number and frequency $( \pm \mathrm{SD})$ of DCs expressing CD70 (D) or CD86 (E). Data are representative of 2-independent experiments ( $\mathrm{n}=3-4$ mice/group) $\left({ }^{*} \mathrm{P}<.05,{ }^{* *} \mathrm{P}<.01,{ }^{* * * *} \mathrm{P}<.0001\right.$ by one-way ANOVA). 
Figure 2.8
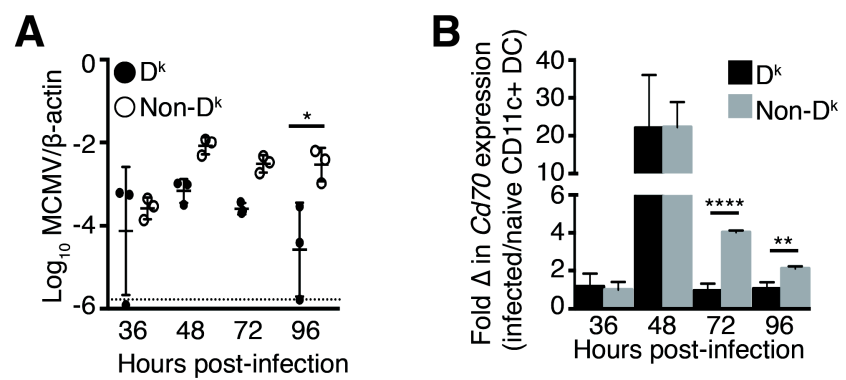

C $\quad-\mathrm{D}^{\mathrm{N}} \mathrm{D}^{\mathrm{N}}-\mathrm{D}^{\mathrm{k}}$
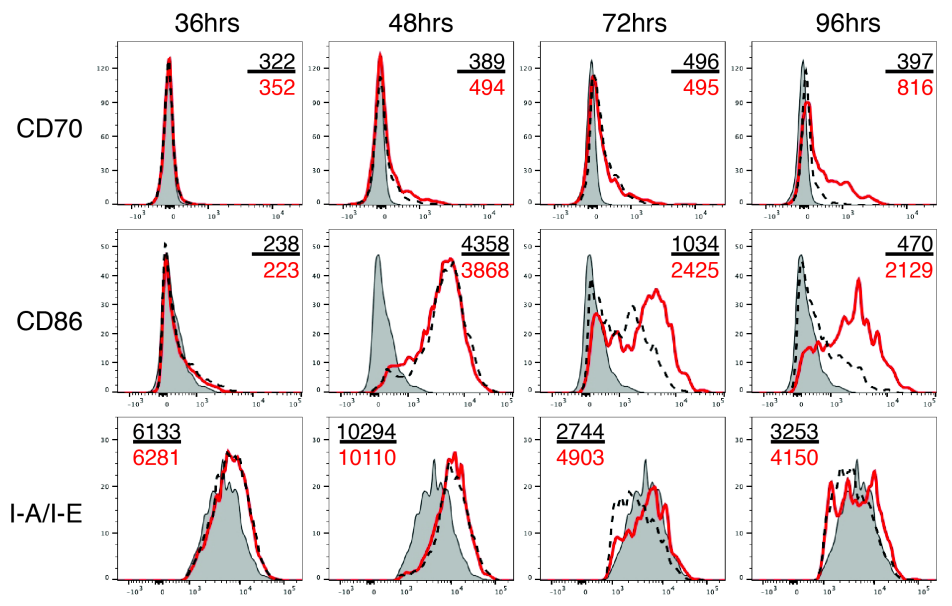

D

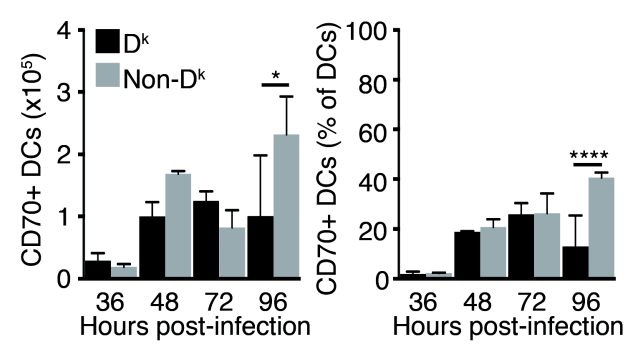

E

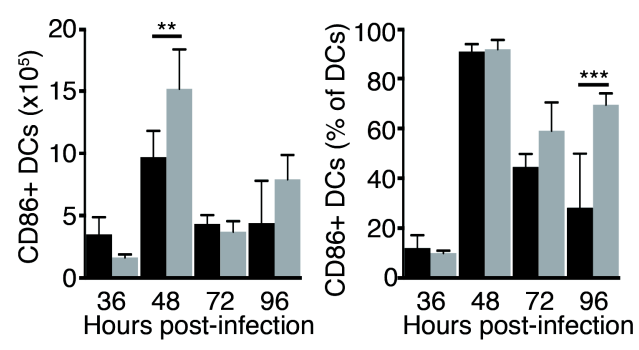




\section{Inefficient NK-mediated control of MCMV results in delayed and CD27- independent accumulation of KLRG1+ SLECs}

Given CD70's protracted expression in DC due to inefficient virus control, we pursued its effect on CD8+ T cells later during infection. As in Figure 2.6, total and antigen-experienced CD8+ T cells were again less abundant in infected $(\mathrm{d} 6)$ non- $\mathrm{D}^{\mathrm{k}}$ mice (Fig. 2.9A-D). As a result, deficits in SLECS and MPECs were also observed, regardless of CD27 expression (Fig. 2.9E, 2.9F). However, by d 8 CD3+ and CD8+ T cells in both WT and $\mathrm{KO}$ non- $\mathrm{D}^{\mathrm{k}}$ mice were on par with $\mathrm{T}$ cells in $\mathrm{D}^{\mathrm{k}}$ mice (Fig. 2.9B, 2.9C). Moreover, as the antigen-experienced CD8+T cells in $D^{k}$ mice declined at $d 8$, in non- $\mathrm{D}^{\mathrm{k}}$ mice they significantly increased (Fig. 2.9A, 2.9D). Even more striking, KLRG1+ SLEC differentiation increased more than 2-fold in both WT and KO non-D ${ }^{\mathrm{k}}$ mice (Fig. 2.9A, 2.9E). These data suggested that a lack of efficient MCMV control, rather than CD70-CD27 signaling, led to significantly altered CD8+ T-cell differentiation. On the other hand, CD127+ KLRG1-CD8+ T cells decreased as the population of KLRG1+CD127- cells increased, but this had little effect on the total number of CD127+CD8+ T cells (Fig. 2.9A, 2.9F). Significant contractions of the SLEC population in $\mathrm{D}^{\mathrm{k}}$ mice and compensatory growth in the number of differentiated CD127+ MPECs at d 8 were also observed. Moreover, this bias toward SLEC contraction and MPEC accrual was reversed in CD27 WT-D ${ }^{\mathrm{k}}$ mice through specific depletion of G2+ NK cells prior to MCMV infection (Fig. 2.9G), which confirms a specific role of $\mathrm{D}^{\mathrm{k}}$ licensed G2+ NK cells in shaping CD8+ T-cell immunity. Together these data therefore suggested that the balance of licensed NK cell-mediated virus resistance distinguished the patterns of CD8 $+\mathrm{T}$ cell differentiation in response to MCMV infection. Whereas more 
efficient NK-mediated virus control coincided with earlier CD8+ T-cell priming and SLEC formation, higher viral burden corresponded to later CD27-independent accumulation of KLRG1+ SLECs and fewer MPECs. 
Figure 2.9. Inefficient NK-mediated control of MCMV results in delayed and CD27independent accumulation of KLRG1+ SLECs. Representative contour plots show the frequency accumulation of antigen experienced CD8+ T cells (top) and the differentiation of antigen experienced CD8+ T cells into SLECs and MPECs (bottom) for CD27 WT-D ${ }^{\mathrm{k}}$, CD27 WT, and CD27 KO mice at 6 and $8 \mathrm{~d}$. (B-F) Bar graphs display the mean ( \pm SD) number of total CD3 $+\mathrm{T}$ cells $(\mathrm{B})$, total $\mathrm{CD} 8+\mathrm{T}$ cells $(\mathrm{C})$, antigen experienced CD8 $+\mathrm{T}$ cells (D), SLECs (E), and MPECs (F). (G) CD27 WT-D ${ }^{\mathrm{k}}$ mice were treated with isotype or depleting mAb 4D11 ( $\alpha$-Ly49G2) prior to MCMV infection. Bar graphs show the mean $( \pm \mathrm{SD})$ frequencies of total CD8 + T cells, CD44+ CD11c + CD8 + T cells, SLECs, and MPECs. Data are representative of 2 independent experiments ( $n=3-4$ mice/group). Statistical comparisons were calculated using two-way ANOVA $(* \mathrm{P}<.05, * * \mathrm{P}<.01$, $* * * \mathrm{P}<.001, * * * * \mathrm{P}<.0001)$. 
Figure 2.9
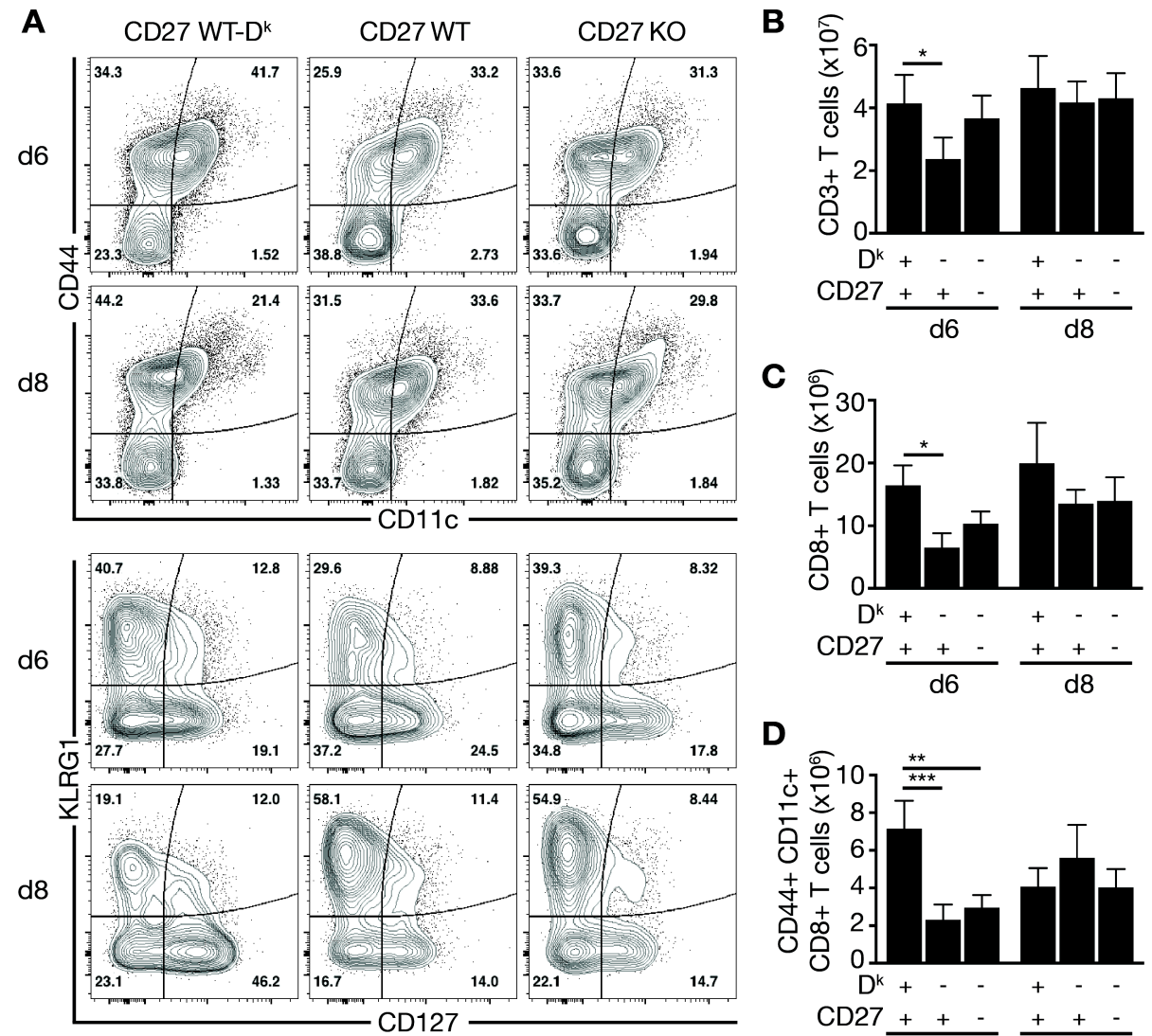

C

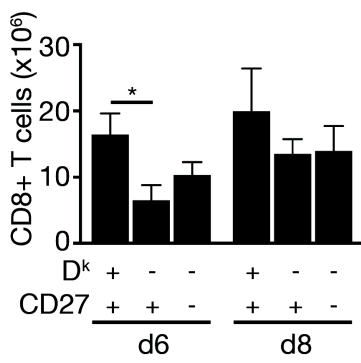

D

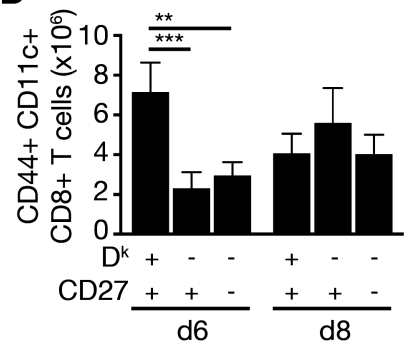

E

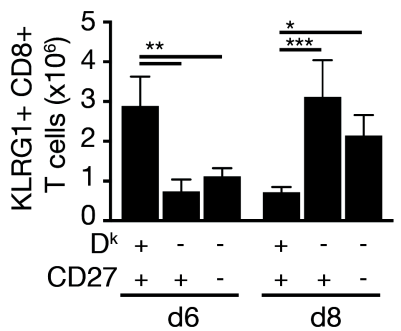

F

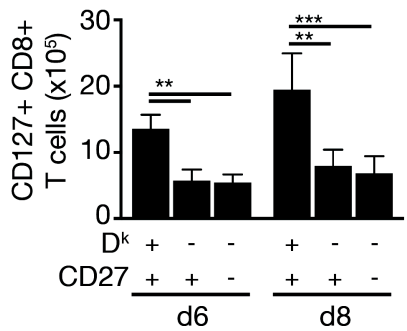

G

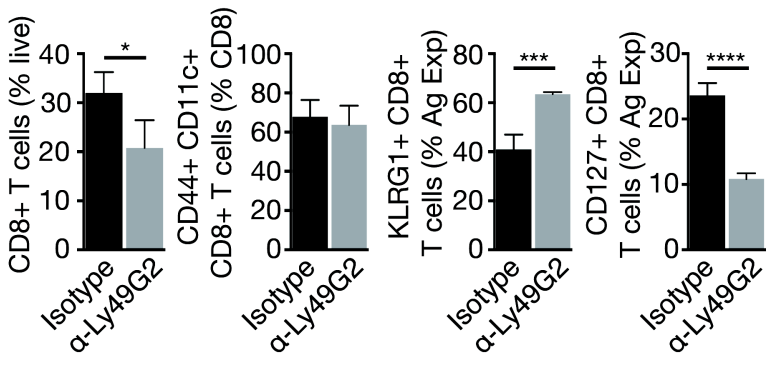


Although CD27 stimulation has been implicated as a primary mediator of memory conversion in CD8+ T cells $(155,235,236,254), \mathrm{CD} 70-\mathrm{CD} 27$ interactions have also been proposed to have opposing effects on CD8+ T cells, depending on the chronicity of infection $(152,255)$. Penaloza-MacMaster et al. (152) demonstrated that CD70-CD27 interactions are vital for priming CD8+ T cells during acute LCMV Armstrong infection, but that persistent CD70 signaling during chronic LCMV clone-13 infection actually decreases CD8 $+\mathrm{T}$ cell accumulation by $\mathrm{d} 21$. Similarly, prolonged CD27 stimulation during chronic LCMV has also been demonstrated to increase CD4+ T cell production of IFN- $\gamma$ and TNF- $\alpha$, both of which disrupt splenic architecture and interfere with viral clearance by neutralizing antibodies (255). Given the prolonged exposure to active viral replication, it is possible that persistent CD27 signaling due to ineffective NK-mediated virus control is detrimental to CD8+ T cell survival and memory conversion.

To investigate if $\mathrm{CD} 27$ hinders the generation of memory $\mathrm{CD} 8+\mathrm{T}$ cells in response to MCMV in non- $\mathrm{D}^{\mathrm{k}}$ mice, we infected $\mathrm{WT}$ and $\mathrm{CD} 27 \mathrm{KO} \mathrm{D}^{\mathrm{k}}$-disparate mice with MCMV and followed the infections to d 21. Surprisingly, two major phenotypes were noted: First, CD27 expression had little impact on frequencies of CD3+ or CD8+ T cells in peripheral blood from $\mathrm{D}^{\mathrm{k}}$ or non- $\mathrm{D}^{\mathrm{k}}$ mice (Fig. 2.10A-C). However, the frequency of circulating M45-specific CD8+ T cells was decreased in CD27 KO mice relative to WT mice (Fig. 2.10D). Hence, CD27 was required to maintain antigenspecific CD8+ T cells after virus clearance from spleen, which was independent of the extent of NK-mediated MCMV resistance or a robust CD8+ $\mathrm{T}$ cell response during acute infection. Second, the frequencies of SLEC and MPEC differentiation within the 
respective populations of virus-specific CD8 $+\mathrm{T}$ cells revealed that effector $\mathrm{T}$ cell populations expressing KLRG1 or CD127 trended within $\mathrm{D}^{\mathrm{k}}$ and non- $\mathrm{D}^{\mathrm{k}}$ mice (Fig. 2.10A, 2.10E, 2.10F). Regardless of CD27 expression, mice with licensed NK-cell control tended to skew towards CD127+ memory CD $8+\mathrm{T}$ cell responses, whereas mice without licensed NK cell control biased CD8+ T cells towards a KLRG1+ effector CD8+ $\mathrm{T}$ cell fate. Thus, these results indicated that while CD70-CD27 interactions improve the survival and accumulation of antigen-specific CD8 $+\mathrm{T}$ cells, differentiation skewing is substantially influenced by early viral control mediated by NK cells. 
Figure 2.10. CD27 is critical for maintaining virus-specific of CD8+ $T$ cells after acute MCMV infection. CD27 WT-D ${ }^{\mathrm{k}}, \mathrm{CD} 27 \mathrm{WT}$, and CD27 KO mice were infected with MCMV, and peripheral blood was sampled at $21 \mathrm{~d}$. (A) Representative contour plots display the frequencies of SLECs and MPECs for $\mathrm{D}^{\mathrm{k}}$-disparate CD27 WT and CD27 KO mice. (B-F) The bar graphs display mean $( \pm S D)$ frequencies of total $C D 3+(B)$, $\mathrm{CD} 8+(\mathrm{C})$ and tetramer+ CD8+ T cells. Graphs for SLECs (E) and MPECs (F) are shown as proportions of M45-tetramer+ populations $(n=4-5$ mice per/group). Statistical comparisons were calculated using one-way ANOVA $(* \mathrm{P}<.05, * * \mathrm{P}<.01, * * * \mathrm{P}<.001$, $* * * * \mathrm{P}<.0001)$ 
Figure 2.10

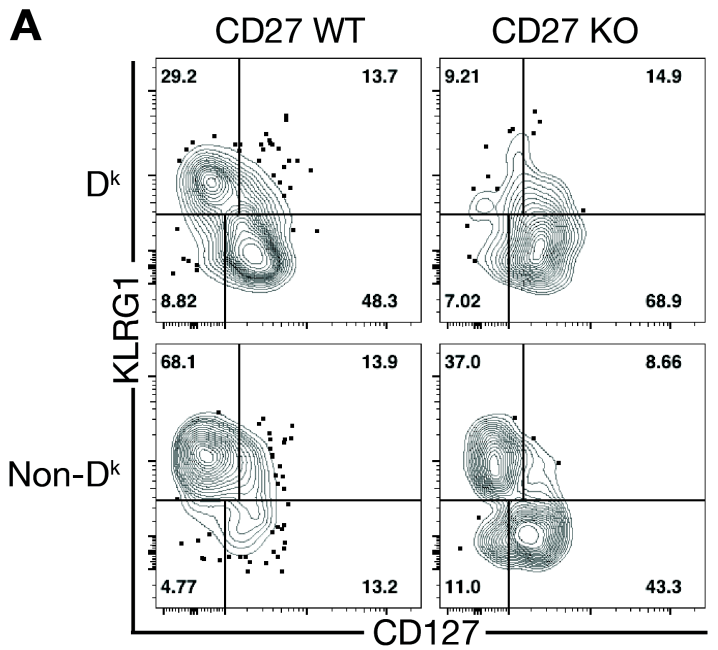

B

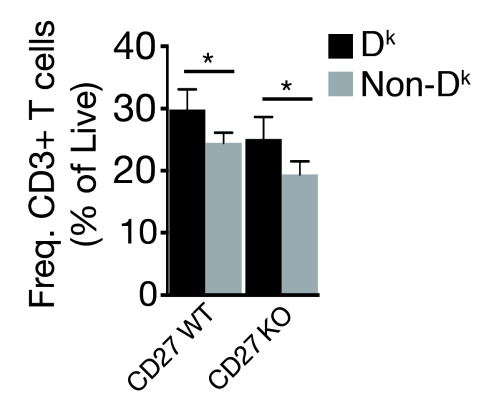

E

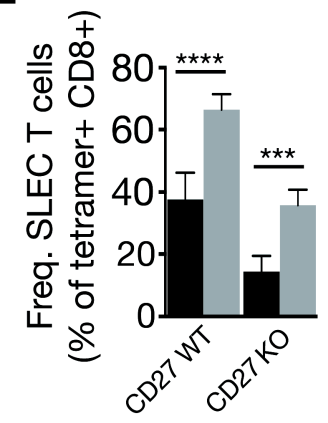

C

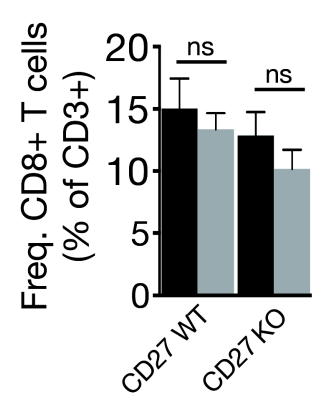

F

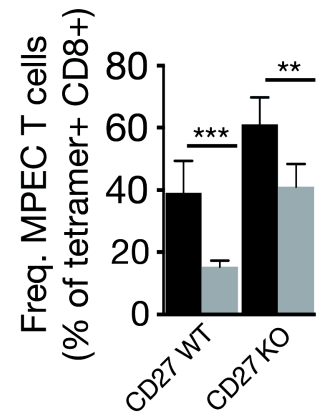

D

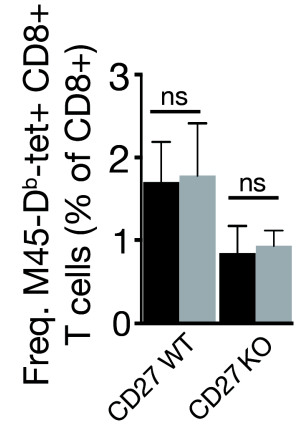




\section{Discussion}

The ability to mount highly functional and specific NK cell responses to viral infection is integral to protecting hosts against repeated virus challenges $(37,229)$. In addition to the direct antiviral properties of NK cells, these innate lymphocytes also play critical roles in preventing excessive immunopathology $(13,18,227)$, preserving lymphoid organ architecture (212), and licensing DCs for improved priming of T cells (20-22). Whether the impacts of NK cells on CD8+ T-cell immunity are ultimately beneficial or detrimental to protective host immunity is still debated, and likely depends on host- and virus-specific contexts $(11,12,256)$. Particularly for NK cells, host genetic factors yield considerable influence over the cues to which the NK cells can respond $(27,54,75)$, and likewise, the manner in which the virus manipulates infected target cells informs the type and quality of NK reactivity (6).

Here we investigated pathways through which licensed NK cells responding to MCMV infection improve adaptive CD8+ $\mathrm{T}$ cell immunity. We found that similar to other mouse models of efficient NK-mediated MCMV resistance (234, 235), CD27 and CD28 co-stimulatory interactions were necessary to promote accumulation of CD8+ T cells by $\mathrm{d} 6$. Moreover, CD70 specifically impacted the proportional accumulation of CD8 $+\mathrm{T}$ cells and shaped effector cell differentiation. Given the critical role for CD27 in

driving CD8+ T cell responses in mice with $\mathrm{D}^{\mathrm{k}}$-licensed NK cells and the minimal effect of CD27 deficiency in mice with much less efficient virus resistance, we expected licensed NK cells responding to MCMV to specifically induce of CD70 on DCs. However, we found that CD70 was equally upregulated on splenic DCs early after infection, even in the absence of licensed G2+ NK cells. Thus, rather than influencing 
the expression of the CD70 on splenic DCs, licensed G2+ NK cells determined the set of cues necessary for priming and differentiating acute CD8+ effector T cells.

$\mathrm{CD} 8+\mathrm{T}$ cells are exposed to a variety of signals during viral infection that influence their priming and differentiation. These signals include (i) the strength and duration of antigen recognition by the TCR (signal 1), (ii) co-stimulatory interactions at the immunological synapse (signal 2), and (iii) cytokines in the inflammatory milieu (signal 3) [reviewed in (116)]. Tight regulation and coordination of signal 2 is key to driving a controlled cytotoxic T-cell response without inducing anergy or immunopathology $(153,257)$. While many of these co-stimulatory pathways are shared across diverse viral infections, several reports have acknowledged that distinct families of viruses as well as the relative persistence of viral infections can significantly impact Tcell reliance and host-benefits from certain co-stimulatory cues $(146,149,152,255,258)$. Croft and colleagues (258) previously correlated the virulence of recombinant vaccinia strains with increased utilization of CD27 and OX40 co-stimulatory pathways. In that study, deficiencies in CD27 or OX40 receptors were only detrimental when the host was challenged with highly virulent vaccinia virus. In related work, CD70-CD27 interactions were shown to be either critical or dispensable for $\mathrm{CD} 8+\mathrm{T}$-cell immunity depending on whether mice were infected with acute LCMV Armstrong or chronic LCMV clone-13, respectively (152). It was hypothesized that while CD70-CD27 co-stimulation was vital for immune responses to acute LCMV with low antigenic exposure, chronic LCMV infection likely induced alternative co-stimulatory pathways that precluded dependence on CD70. Moreover, over-stimulation of CD27 has actually been shown to impede 
adaptive immune responses during chronic viral infection and prolong the time to viral clearance $(152,255)$.

Here, rather than comparing the impacts of different virus strains (e.g. Armstrong vs. clone-13) on the outcomes of co-stimulatory signaling pathways, we varied the host models and their ability to efficiently control infection. We predicted that prolonged exposure to MCMV in mice without efficient licensed NK cell control would increase the number and diversity of co-stimulatory signals utilized by antigen-specific CD8+ T cells. Similar to the studies with LCMV Armstrong and clone-13, the efficiency of viral control was a chief determinant of acute $\mathrm{CD} 8+\mathrm{T}$ cell dependence on $\mathrm{CD} 27$. Whereas $\mathrm{D}^{\mathrm{k}}$ mice were critically dependent on $\mathrm{CD} 27$ for enhancement of CD8 $+\mathrm{T}$ cell accumulation, mice without $\mathrm{D}^{\mathrm{k}}$ demonstrated a delayed but $\mathrm{CD} 27$-independent accrual of $\mathrm{T}$ cells. While conditions of prolonged viral antigen exposure likely induced additional co-stimulatory or inflammatory pathways that could drive acute CD8+ T cell accumulation and differentiation, CD27 was still integral for establishing CD8+ T memory cells in both $\mathrm{D}^{\mathrm{k}}$ and non- $\mathrm{D}^{\mathrm{k}}$ mice, and did not provoke the negative effects previously seen with LCMV clone-13 (152). Thus, regardless of the efficiency of viral control, CD27 plays a critical role in shaping memory differentiation and survival, consistent with previous studies $(155,156,235,246)$. However, when MCMV is efficiently dampened via licensed NK cells and putatively less antigen and inflammation is present, the potency of CD70-CD27 interactions is necessary to promote immunity over tolerance. Future studies are warranted to further explore the long-term impacts of licensed NK-mediated MCMV control on memory $\mathrm{T}$ cells. Given that additional stimuli may be guiding delayed CD8+ T cell priming and that differentiation skewing is informed by the presence of licensed G2+ 
NK cells, we speculate that the early influence of licensed NK cells during MCMV infection may have lasting impacts on host immunity.

As we begin to dissolve the complexities of NK regulation of adaptive immunity, it is clear that the 'one size fits all' approach is insufficient to explain the diversity of NK cell influences that we observe across disease models. Particularly as we develop and implement immunotherapeutic strategies against infectious diseases and varieties of cancer, it will be important to evaluate the impact of NK cells in host-specific contexts. In genetically diverse inbred mouse strains, we observe a plethora of NK cell influences ranging from vital support for $\mathrm{T}$ cell immunity $(23,24,93,253)$ to cytolytic elimination of activated lymphocytes $(13,16-18,227)$. From studies of human NK cell responses to viral infections - including HCMV, HIV, and HCV - it is understood that specific pairings of NK cell receptors and HLA alleles could influence the extent of viral resistance $(54,75)$. Moreover, such receptor-ligand interactions may directly influence selective DC survival or maturation early during infection (231). In the same vein, while the diversity of $\mathrm{T}$ cell co-stimulatory cues for various models of disease have been well studied (259), it is clear that different viral infections will influence the pathways utilized to promote protective immunity $(149,152,258)$. Still, even with all of these cells and signals in place, host-genetic factors that instruct NK cell responses may consequently shape the impact and necessity of those signals, as demonstrated herein. Thus, in addition to studying the cell types and signals that can foster cell-mediated immunity, we will need to develop a more thorough understanding of early innate immunity and how it affects the immunogenic milieu. 


\section{Chapter III}

Licensed NK cells shape CD8+ T cell memory formation and protective immunity 


\section{Introduction}

NK cells and CD8+ T cells are critical mediators of cell-mediated immunity against acute, persistent, and chronic viral infections. Although historically these effector lymphocyte subsets have been temporally divided into separate phases of the immune response, over the last decade, several studies have identified overlapping features and interactions of $\mathrm{NK}$ and $\mathrm{CD} 8+\mathrm{T}$ cells $(1,11,12,260)$. NK cells are amongst the earliest of effector cells to respond to malignant or virus-infected host cells, utilizing integrated signals from germline encoded activating and inhibitory receptors to survey and discern healthy self from diseased target cells $(36,75)$. In addition to cellular cytotoxicity, NK cells mediate immunoregulatory functions, including (i) release of cell subset and maturation skewing cytokines $(20,22,97)$, (ii) regulation of available antigen for lymphocyte priming $(106,109)$, and (iii) direct lysis of iDCs and activated CD4+ and CD8+ T cells $(12-15,261)$. Previous work with genetically diverse mouse strains and different infectious pathogens has shown that NK cells can confer supportive or suppressive influences on adaptive immune mediators, such as $\mathrm{CD} 8+\mathrm{T}$ cells. However, the precise role of host genetics and NK cell responsiveness to particular pathogens on CD8+ T cell immunity remains incompletely understood.

Cytomegalovirus (CMV) is a prototypical beta-herpesvirus that establishes lifelong, persistent infections in species-specific hosts. Having co-evolved with its host for eons, CMV is remarkably resilient to detection and sterilizing immunity (262). Murine (M)CMV in particular has been instrumental for characterizing the requisite roles of NK and CD8+ T cells in mediating viral resistance. Studies in both immunodeficient and immunodepleted mice have demonstrated specific and non-redundant roles for these 
cell-mediated immune responses to MCMV infection $(3,42)$. Likewise, analyses of MCMV pathogenesis and specific immunoevasins have highlighted key immune pressure points provided by both NK and CD8+ T cells (reviewed in (6) and (262)). Genetically distinct strains of mice demonstrate varying degrees of natural resistance to MCMV and unique mechanisms of NK cell-mediated virus control (38). Specific pairings of MHC haplotypes and NK cell receptors in particular can significantly influence mechanisms of NK cell activation and viral resistance $(39,41,263,264)$. Thus, the manner and degree of viral control conferred by NK cells is likely to yield significant impacts on the magnitude, differentiation, and protective capacity of adaptive immune responses.

Correlations between the efficiency of acute MCMV control by NK cells and the kinetics of $\mathrm{CD} 8+\mathrm{T}$ cell accumulation after infection have already been well established $(23,24)$. In C57Bl/6 mice, $\mathrm{NK}$ cells with the activating receptor Ly49H expressed, provide critical MCMV resistance (47) via rapid recognition and lysis of infected target cells expressing viral peptide m157 $(48,49)$. Early viral control by Ly49H+ NK cells additionally dampens excessive type I IFN production, which improves the recovery of DCs and subsequently accelerates CD8+ T-cell accrual (23). However, accelerated T cell immunity comes at a cost, as $\mathrm{Ly} 49 \mathrm{H}+\mathrm{NK}$ cells may limit antigen-presentation via lysis of MCMV-infected DCs, and hasten T-cell contraction (109). As a result, CD8+ T cells displayed reduced cytotoxicity and cytokine production by $\mathrm{CD} 8+\mathrm{T}$ cells in the weeks following infection $(106,109)$.

MHC I $\mathrm{D}^{\mathrm{k}}$ is a different major determinant of MCMV resistance. It is also a licensing ligand for NK cells expressing the inhibitory receptor, Ly49G2 (G2). Consequently, $\mathrm{D}^{\mathrm{k}}$-licensed $\mathrm{G} 2+\mathrm{NK}$ cells are critical to virus control, and either depletion 
of or interference with normal licensing on self-MHC I $\mathrm{D}^{\mathrm{k}}$ is sufficient to impair their capacity to mediate viral control in otherwise resistant mouse strains $(24,68,71,78)$. Interestingly, $\mathrm{D}^{\mathrm{k}}$-licensed G2+ NK cells were also found to protect and regulate DCs, which could promote CD8+ T-cell priming without altering $\mathrm{T}$ cell contraction and further influence early T cell differentiation (24)[Teoh et al. Manuscript accepted. J Immunol (2016)]. However, the effect of licensed NK-mediated virus control on CD8+ T cell memory formation and maintenance has not been previously studied. Thus, here we examined the effect of licensed NK cells on the differentiation, long-term maintenance, and functional performance of virus-specific CD8+ T cells during MCMV infection. 


\section{Results}

\section{Licensed NK cell control of MCMV specifically regulates CD8+ T cells memory differentiation}

To assess the effects of $\mathrm{D}^{\mathrm{k}}$-licensed $\mathrm{NK}$ cell control on $\mathrm{CD} 8+\mathrm{T}$ cell differentiation and memory formation, we infected $\mathrm{B} 6 . \mathrm{NKC}^{1}$ congenic mice with MCMV and assessed CD8+ T-cell accrual and differentiation. As expected $(24,71), \mathrm{D}^{\mathrm{k}}$ mice readily controlled MCMV (d 6-10), whereas non- $\mathrm{D}^{\mathrm{k}}$ mice exhibited at least 100 -fold higher virus levels at d 6, and were still detectable at d 10 (Fig. 3.1A). Correspondingly, $\mathrm{D}^{\mathrm{k}}$ mice had higher numbers of total and M45-specific CD8+ T-cells at d 6 (Fig. 3.1B, C). Nonetheless, non- $\mathrm{D}^{\mathrm{k}} \mathrm{CD} 8+\mathrm{T}$ cells caught up by $\mathrm{d} 8$, and both strains contracted $\mathrm{T}$ cells equally by d 10 post-infection. In contrast, we did not observe significant fluctuations in CD4+ $\mathrm{T}$ cells during acute infection in either mouse strain (Fig. 3.1D). The results suggest that licensed NK cell control of MCMV specifically affects CD8+ T cell immunity.

When we further examined CD8 $+\mathrm{T}$ differentiation, we found that $\mathrm{D}^{\mathrm{k}}$ mice had higher numbers and a greater proportion of both KLRG1+ CD127- short-lived effector cells (SLECs) and CD127+ KLRG1- memory precursor effector cells (MPECs) at d 6 (Fig. 3.1E-H). A higher frequency of non-D ${ }^{\mathrm{k}}$ MPECs at d 6 could be due to delayed CD127 down-regulation, consistent with delayed CD8+ T cell accumulation and eventual decrease in CD127 expression (Fig. 3.1G,H). The number and frequency of M45-specific SLECs rapidly declined after $\mathrm{d} 6$ in $\mathrm{D}^{\mathrm{k}}$ mice, and paralleled increasing MPEC numbers and frequency (Fig. 3.1E-G). In contrast, at $\mathrm{d} 8$ when non- $\mathrm{D}^{\mathrm{k}}$ mice still had detectable MCMV, SLECs were significantly amplified, reaching a peak accumulation at least 3- 
fold greater than that of $\mathrm{D}^{\mathrm{k}}$ mice at the same time point during acute infection. Non- $\mathrm{D}^{\mathrm{k}}$ SLECs ultimately declined by d 10, however this decrease was not accompanied by a compensatory increase in MPECs as observed in the $\mathrm{D}^{\mathrm{k}}$ strains. Accordingly, KLRG1 and CD127 median fluorescence intensity (MFI) levels on CD8+ T cells were also shifted (Fig. 3.1H). As the MFI shifts mirrored changes in the tempo and differentiation trends, these data suggested that cell autonomous features typify KLRG1 and CD127 expression during MCMV infection in the $\mathrm{D}^{\mathrm{k}}$-disparate strains. Thus, in addition to accelerating virus-specific CD8 + T cell accrual, efficient licensed NK cell-mediated MCMV control biases T cell differentiation towards MPECs over SLECs.

Interestingly, we observed that SLECs could be further distinguished by their expression of KLRG1 (KLRG1 $1^{\text {hi }}$ and KLRG1 $1^{\text {lo }}$, respectively) (Fig. 3.1E, Fig 3.2). Although we observed that KLRG1 corresponded with SLEC differentiation during MCMV infection, it has been shown that variable KLRG1 expression corresponds with distinct cell fate potentials. In particular, $\mathrm{KLRG} 1^{10}$ cells were found to expand and differentiate into both SLECs and MPECs upon transfer into infection-matched recipients, whereas KLRG $1^{\text {hi }}$ cells remained as terminally differentiated SLECs (135). Hence, we analyzed CD8+ T cells responding to MCMV for KLRG1 expression features. At $\mathrm{d} 6$, both $\mathrm{D}^{\mathrm{k}}$ and non- $\mathrm{D}^{\mathrm{k}}$ mice had 2-fold higher numbers of KLRG $1{ }^{\mathrm{hi}}$ SLECs compared to KLRG $1^{\text {lo }}$ (Fig. 3.1I). The KLRG $1^{\text {hi }}$ to KLRG $1^{\text {lo }}$ index decreased after $d 8$ in $\mathrm{D}^{\mathrm{k}}$ mice, and eventually reached a 1:1 ratio. In striking contrast, the index increased to a ratio of $4: 1$ at $\mathrm{d} 8$ in non- $\mathrm{D}^{\mathrm{k}}$ mice, and remained much higher $(2: 1)$ than in $\mathrm{D}^{\mathrm{k}}$ mice through $\mathrm{d} 10$. Taken together, these data demonstrate unique differentiation pathways for 
CD8+ T effector cells responding to MCMV, which are highly sensitive to the extent of licensed NK cell control.

MCMV M45 is a non-inflationary early protein that has been noted for its antiapoptotic function in infected cells (265). Moreover, M45 elicits a robust, immunodominant $\mathrm{CD} 8+\mathrm{T}$ cell response during acute infection, which is ideal for tracking virus-specific T cells $(209,266,267)$. However, despite its classification as an immunodominant protein, M45 peptide presentation by MHC I $\mathrm{D}^{\mathrm{b}}$ is generally suppressed by MCMV-derived immunoevasins gp40 $(189,268)$ and gp48 $(190)$, so that TCR interactions with M45- $\mathrm{D}^{\mathrm{b}}$ occurs via DC cross-presentation rather than through effector-target interactions (266). Hence, it was unclear whether differences in CD8+ T cell differentiation in our $\mathrm{D}^{\mathrm{k}}$ disparate mice represented a generalized influence of licensed NK cells on all MCMV-experienced effector cells, or whether this effect was limited to M45- ${ }^{\mathrm{b}}$ cross-primed $\mathrm{CD} 8+\mathrm{T}$ cells.

We assessed virus-responsive CD8 $+\mathrm{T}$ cells co-expressing the homing receptor, CD44, and the beta-2 integrin, CD11c $(247,248)$ [Teoh et al. Manuscript accepted. $J$ Immunol (2016)]. The kinetics for total (Fig. 3.3A) and virus-responsive CD44+ CD11c + CD8+ T cells (Fig. 3.3B) paralleled M45-specific CD8+ T-cells, with notable differences in total d 6 accumulation, but similar numbers and contractions 8-10 d postinfection. Likewise, CD44+ CD11c+ SLEC and MPEC differentiation trends mirrored those observed for M45-specific CD8+ T cells in $\mathrm{D}^{\mathrm{k}}$ disparate mice (Fig. 3.3C, D). Moreover, $\mathrm{T}$ cell $\mathrm{CD} 107 \mathrm{a}+$ degranulation and cytokine production upon re-stimulation with M45 and m139 MCMV peptides paralleled the abundance of SLECs (Fig. 3.3E). 
Thus, in addition to M45- $\mathrm{D}^{\mathrm{b}}$ cross-primed T cells, licensed NK cell control of MCMV broadly affected polyclonal CD8+ T cell immunity.

Still, it remained unclear whether licensed G2+ NK cells were specifically required to skew T cell immunity. To test this, we depleted $\mathrm{G} 2+\mathrm{NK}$ cells from $\mathrm{D}^{\mathrm{k}}$ mice before MCMV infection and compared the ensuing T cell responses. As expected, G2+ NK cells were efficiently depleted through d 8 (Fig. 3.3F). Consequently, antigen experienced CD8+ T cells in G2+ NK-depleted mice were skewed as in non- $\mathrm{D}^{\mathrm{k}}$ mice, with an increased frequency of SLECs and a diminished presence of MPECs relative to controls (Fig. 3.3G-J). In aggregate, these results indicated that the licensed G2+ NK response to MCMV has a profound effect on CD8+ T cell differentiation. 
Figure 3.1. Early viral control by licensed NK cells promotes memory precursor differentiation in virus-specific CD8+ T cells. $D^{\mathrm{k}}$-disparate mice were infected with $5 \times 10^{4}$ pfu MCMV (A) Individual spleen viral levels were examined on d 6-10 postinfection. (B-I) T lymphocytes were gated on live, singlet, CD3+ CD19- cells. M45-D ${ }^{\mathrm{b}}$ tetramer + cells were gated on CD8 + T cells. Graphs display the mean number $( \pm \mathrm{SD})$ of total (B) and M45-D -tetramer + CD8 + T cells (C), and total CD4+ T cells per spleen (D). (E) Representative flow plots show tetramer+ gated SLECs (KLRG1+ CD127-) and MPECs (KLRG1- CD127+). (F-G) Graphs show the mean numbers and frequencies ( \pm SD) of splenic SLECs (F) and MPECs (G). (H) Histograms display the median fluorescence intensities for KLRG1+ and CD127+ tetramer+ cells. (I) Graph represents the mean ratios of KLRG $1^{\text {hi }}:$ KLRG $1^{\text {lo }}$ SLECs. Data are representative of 3 independent experiments ( $\mathrm{n}=3-4$ mice/group). Statistical analyses were performed using two-way ANOVA $(* \mathrm{P}<.05, * * \mathrm{P}<.01, * * * \mathrm{P}<.001, * * * * \mathrm{P}<.0001)$. 
Figure 3.1
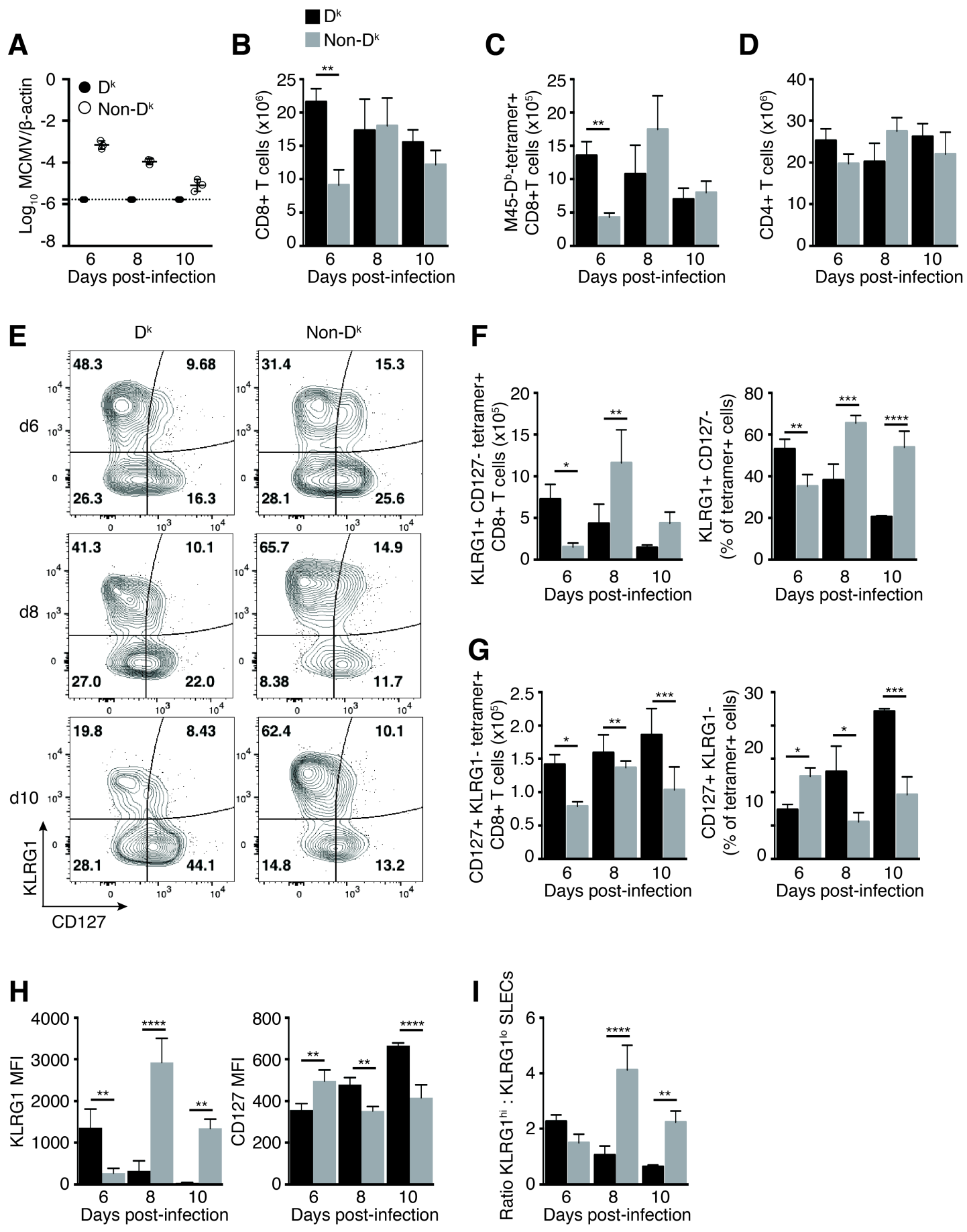
Figure 3.2. Median fluorescence intensity of KLRG1 identifies two distinct CD8+ T effector cell populations. (A) Representative flow plots were gated on live, singlet, CD3+ CD19-, CD8+, M45-D ${ }^{\text {b}}$-tetramer+, KLRG1+ CD127- T cells. KLRG1+ CD8+ T cells were further gated on KLRG $1^{\text {hi }}$ and $\mathrm{KLRG} 1^{\text {lo }} \mathrm{CD} 8+\mathrm{T}$ cells. (B) Graphs represent the mean $( \pm \mathrm{SD})$ frequency of KLRG1 $1^{\text {hi }}$ (top) and KLRG1 ${ }^{\text {lo }}$ (bottom) cells for the respective mouse strains. Data are representative of at least 3-independent experiments ( $\mathrm{n}=3-4 \mathrm{mice} /$ group). Statistics were calculated using one-way ANOVA $(* * * * \mathrm{P}<.0001)$. 
Figure 3.2

A
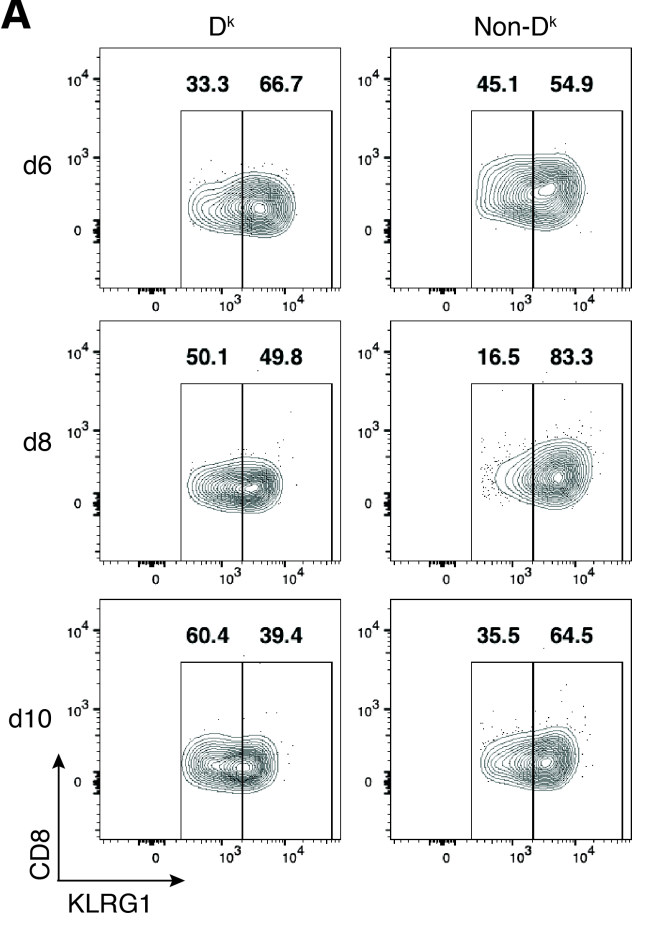

B
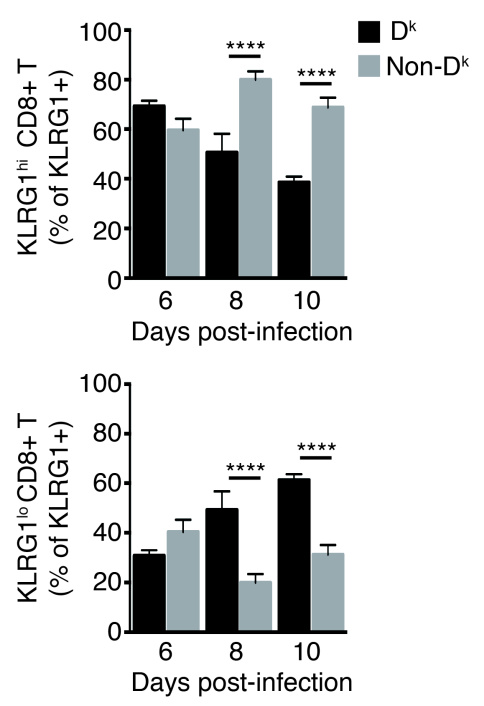
Figure 3.3. Licensed NK cells regulate differentiation of virus-experienced cells. $D^{k}$ disparate mice were infected as described in Fig. 3.1. (A-D) Graphs represent the mean number $( \pm \mathrm{SD})$ of total $(\mathrm{A})$ and virus-experienced $\mathrm{CD} 8+\mathrm{T}$ cells $(\mathrm{B})$, as well as the mean number ( \pm SD) of antigen experienced SLECs (C) and MPECs (D). (E) Graphs show the mean number $( \pm \mathrm{SD})$ of CD8 $+\mathrm{T}$ cells expressing CD107a, IFN $\gamma$ and TNF $\alpha$ upon peptide re-stimulation with M45 and m139. (F-I) $\mathrm{D}^{\mathrm{k}}$ mice were treated with G2+ NK celldepleting $\mathrm{mAb}$ or isotype controls and subsequently infected with MCMV. Peripheral blood mononuclear cells (PBMCs) were isolated and examined on d 8. Graphs represent the mean $( \pm$ SD) frequency of total (left) and G2 + NK cells (right) $(F)$, CD8 + T cells $(G)$, CD44+ CD11c + T cells (H), SLECs (I), and MPECs (J). (A-J) Data are representative of 3 independent experiments ( $n=3-4$ mice per group). Statistical analyses were performed using two-way ANOVA or unpaired Student's t-tests $(* \mathrm{P}<.05, * * \mathrm{P}<.01, * * * \mathrm{P}<.001)$. 
Figure 3.3

A

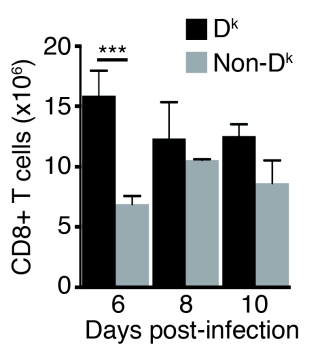

E

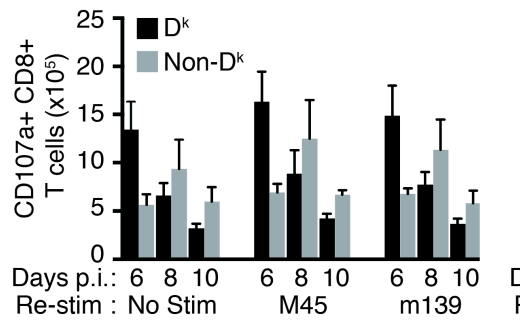

C

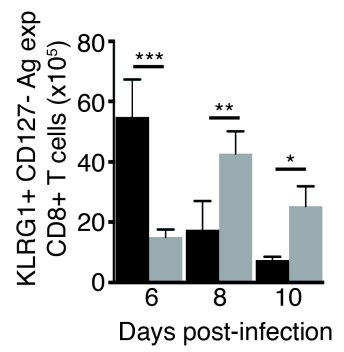

D

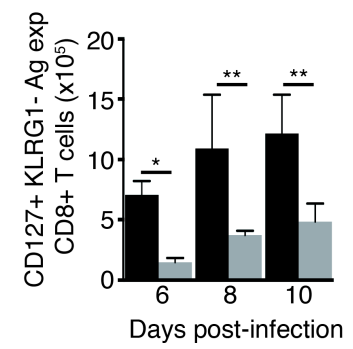

F
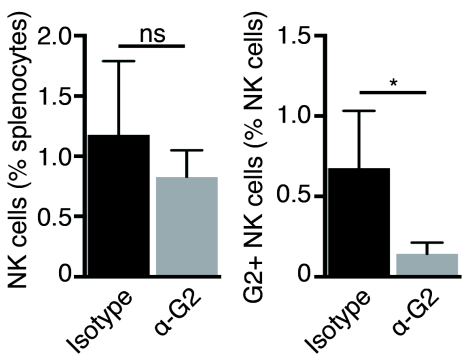

G

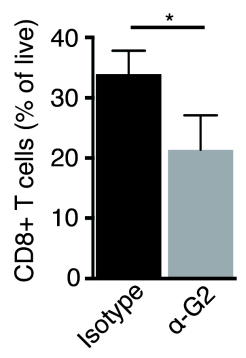

H

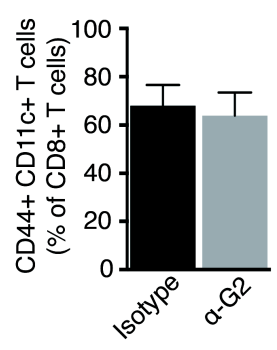

I

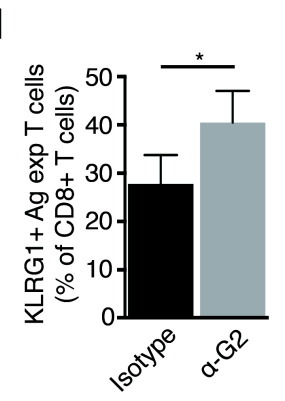

J

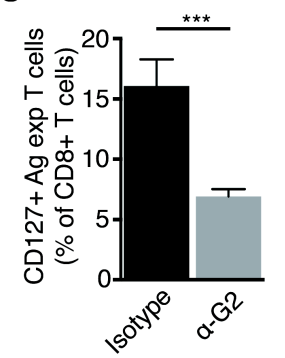




\section{MCMV-specific CD8+ memory $\mathrm{T}$ cell differentiation is IL-10 dependent}

We next examined pathways through which licensed G2+ NK cells might influence CD8+ T cell differentiation. We reasoned that the dose of acute MCMV infection and the efficiency of NK cell-mediated viral control may be key determinants of T cell differentiation fate. We have observed that high dose MCMV infection in $\mathrm{D}^{\mathrm{k}}$ mice delays splenic DC recovery similar to non- $\mathrm{D}^{\mathrm{k}}$ mice (Nash, WT and Brown, MG, unpublished data), which suggested that DC recovery, in addition to virus-induced inflammation, may influence $\mathrm{T}$ cell priming. To test this, we performed MCMV dose response experiments in $\mathrm{D}^{\mathrm{k}}$-disparate mice. We found that 100 -fold more MCMV $\left(10^{4} \rightarrow 10^{6} \mathrm{pfu}\right)$ was required to yield spleen viral levels in $\mathrm{D}^{\mathrm{k}}$ mice that either matched or surpassed those observed in non- $\mathrm{D}^{\mathrm{k}}$ mice (d 8) (Fig. 3.4A). Correspondingly, infection of $\mathrm{D}^{\mathrm{k}}$ mice with an augmented dose of MCMV caused a reduced accumulation of total and virus-responsive $\mathrm{CD} 8+\mathrm{T}$ cells similar to that seen in non- $\mathrm{D}^{\mathrm{k}}$ mice (Fig. 3.4B-F). Despite this, the extent of terminal effector cell differentiation as measured by KLRG1 expression remained consistent with $\mathrm{D}^{\mathrm{k}}$ groups infected with lower doses of MCMV, whereas non- $\mathrm{D}^{\mathrm{k}}$ mice exhibited a 3.5-fold increase for this index (Fig. 3G). Thus, $\mathrm{D}^{\mathrm{k}}$ licensed NK cells impact T cell priming and differentiation on two levels: (i) Efficient NK-mediated viral control hastens recovery of DCs, which can accelerate accumulation of CD8+ T cells during infection; and (ii) additional factors of licensed NK-mediated resistance - independent of viral control - shape effector cell differentiation.

Previously, we discovered that licensed NK cell control of MCMV lessened the

duration of DC display of CD86 and CD70, whereas non- $\mathrm{D}^{\mathrm{k}}$ mice sustained expression of these co-stimulatory ligands [Teoh et al. Manuscript accepted. J Immunol. (2016)]. In 
addition to this, key roles for anti-inflammatory cytokines, such as IL-10, and controlled co-stimulation in guiding CD8+ memory $\mathrm{T}$ cell differentiation have been demonstrated $(133,269,270)$. Although co-stimulation is generally required to initiate $\mathrm{T}$ cell responses, restraining the duration of co-stimulatory and inflammatory signaling has been proposed to foster MPEC differentiation over terminal SLEC formation. Given the decreased display of co-stimulatory ligands and enhanced conversion to memory precursors in $\mathrm{D}^{\mathrm{k}}$ mice, the results suggested that IL-10 may contribute to licensed NK cell regulation of memory $\mathrm{T}$ cell differentiation during MCMV infection.

To investigate this, we neutralized IL-10 receptor signaling in $\mathrm{D}^{\mathrm{k}}$ mice during MCMV infection. Despite that spleen weights and splenocyte numbers were significantly increased in IL-10R-blocked mice compared to $\mathrm{D}^{\mathrm{k}}$ and non- $\mathrm{D}^{\mathrm{k}}$ controls, IL10R blockade did not impede licensed NK cell-mediated viral control at d 8 (data not shown). Likewise, total T cell accumulation, including M45-specific CD8+ T cells, was heightened in the absence of IL-10R signaling, but the frequencies of tetramer+ cells did not vary amongst groups (Fig. 3.5A-D). Interestingly, IL-10R blockade in $\mathrm{D}^{\mathrm{k}}$ mice phenocopied virus-specific $\mathrm{CD} 8+\mathrm{T}$ cell differentiation patterns in non- $\mathrm{D}^{\mathrm{k}}$ mice, with increased frequencies of SLECs and decreased proportions of MPECs (Fig. 3.5E-G). Although the absolute numbers of MPECs were similar for both $D^{k}$ groups, we attributed these similarities to the increased accumulation of total T cells in $\alpha$-IL-10R-treated mice, since frequencies of MPECs were significantly lower than in $\mathrm{D}^{\mathrm{k}}$ mice. These data indicate that $\mathrm{D}^{\mathrm{k}}$-licensed NK cell enhancement of MPEC differentiation during MCMV infection is dependent on early IL-10 signaling. 
Although we have not determined whether IL-10 acts directly on CD8+ T cells or if it has a dominant role in dampening DC maturation as seen previously (270), we have preliminarily investigated the impact of IL-10R on the inflammatory environment (d 8). Similar to the impact on T cells, IL-10R blockade elevated the frequency and number of total DCs during MCMV infection (Fig.3.6B, data not shown). In addition, both CD70 and CD86 were also expressed at substantially higher levels on DCs in IL-10R blocked and non- $\mathrm{D}^{\mathrm{k}}$ mice compared to $\mathrm{D}^{\mathrm{k}}$ mice, even though we did not observe differences in MHC II expression (Fig. 3.6A). IL-10R-treated mice also had higher frequencies of DCs expressing either $\mathrm{CD} 70$ or $\mathrm{CD} 86$ relative to either of the $\mathrm{D}^{\mathrm{k}}$ and non- $\mathrm{D}^{\mathrm{k}}$ control groups (Fig. 3.6C,D). Thus IL-10 signaling may be part of a critical anti-inflammatory axis needed to restrain DC maturation and guide MPEC differentiation in the wake of viral control.

We next explored the impact of IL-10 on monocytes during MCMV infection. In line with sustained maturation of DCs, we observed a 1.5 to 2 -fold increase in total $\mathrm{CD} 115+\mathrm{CD} 11 \mathrm{~b}+$ monocytes in IL-10R-blocked $\mathrm{D}^{\mathrm{k}}$ mice, and non- $\mathrm{D}^{\mathrm{k}}$ mice relative to $\mathrm{D}^{\mathrm{k}}$ control mice (Fig. 3.6E,F). Furthermore, less than $2 \%$ of the splenic monocytes in $\mathrm{D}^{\mathrm{k}}$ mice expressed molecular pDC antigen-1 (mPDCA-1), a specific marker of type I IFNproducing $\mathrm{pDCs}$ that is broadly up-regulated on most cell types following stimulation with IFN (271)[Nash et al. Manuscript submitted]. In comparison, more than $99 \%$ of total monocytes in $\alpha$-IL-10R and non- $\mathrm{D}^{\mathrm{k}}$ groups expressed mPDCA-1 (Fig. 3.6G), which suggested that sustained inflammatory signaling was present in these groups.

Prior studies have identified specific impacts of distinct monocyte subsets on host-immunity to MCMV, including inflammatory monocyte restriction of CD8+ effector 
T cell development (272) and patrolling monocyte dissemination of virus to peripheral organs (273). Hence, we examined whether specific monocyte subsets varied amongst $\mathrm{D}^{\mathrm{k}}$-disparate and $\alpha$-IL-10R-treated groups. Significant distinctions in proportional monocyte populations were observed between $\mathrm{D}^{\mathrm{k}}$ mice, IL-10R-blocked, and isotypetreated non- $\mathrm{D}^{\mathrm{k}}$ mice. Remarkably, $\alpha-$ IL-10R and non- $\mathrm{D}^{\mathrm{k}}$ groups exhibited a 1.5-2-fold greater frequency of patrolling monocytes (GR $\left.1^{10} \mathrm{CD} 11 \mathrm{c}-\right)$ for IL-10R-blocked and non$D^{k}$ mice compared to $D^{k}$ mice (Fig. 3.6H). In contrast, $D^{k}$ mice maintained a significantly greater proportion of inflammatory monocytes (GR $\left.1^{\text {inthi }} \mathrm{CD} 11 \mathrm{c}-\right)$ than either of the other groups (Fig. 3.6I). This bias towards inflammatory or patrolling monocytes suggests that IL-10R signaling not only influences the duration of splenic inflammation during MCMV infection, but also the quality and type of monocyte subsets present, which could shape different host immune responses. Moreover, the similarities amongst the cellular profiles examined in IL-10R-blocked and non- ${ }^{\mathrm{k}}$ control groups $(\mathrm{CD} 8+\mathrm{T}$ effector cells, DCs, patrolling monocytes) further supports a critical role for IL-10 in guiding CD8+ T memory cell differentiation in mice with $\mathrm{D}^{\mathrm{k}}$-licensed NK cell-mediated viral control. 
Figure 3.4. Scaled infection dose delays $T$ cell accumulation but does not skew differentiation. $\mathrm{D}^{\mathrm{k}}$ mice were infected with increased doses of $\operatorname{MCMV}\left(10^{4}, 10^{5}\right.$, and $10^{6} \mathrm{pfu}$ ) and compared to non- $\mathrm{D}^{\mathrm{k}}$ mice infected with $10^{4} \mathrm{pfu}$ MCMV. (A) Shown are the splenic viral levels for the indicated mouse groups. (B) Representative contour plots display the d 8 post-infection frequencies of virus-experienced CD11c+CD44+ CD8+ T cells (left) and SLECs and MPECs (right). (C-F) Bar graphs display the mean number ( \pm SD) of total CD8+ T cells (C), antigen-experienced CD8+ T cells (D), SLECs (E), and MPECs (F). (G) Graph displays the mean ratio of KLRG1 ${ }^{\text {hi }}: \mathrm{KLRG}^{\text {lo }}$ SLECs. Data are representative of a single independent experiment ( $\mathrm{n}=2$ mice/group). 
Figure 3.4

A

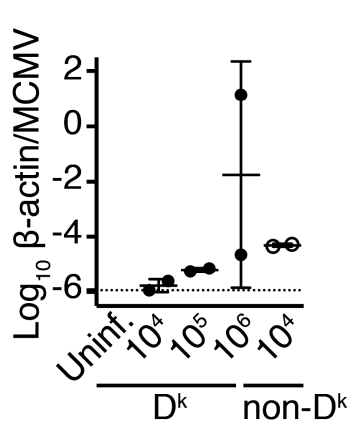

C

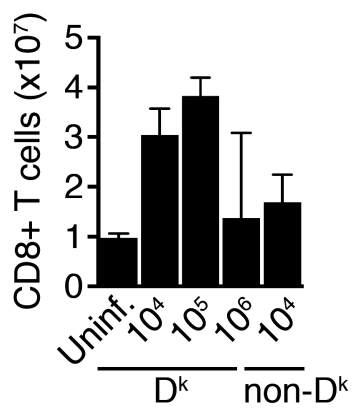

D

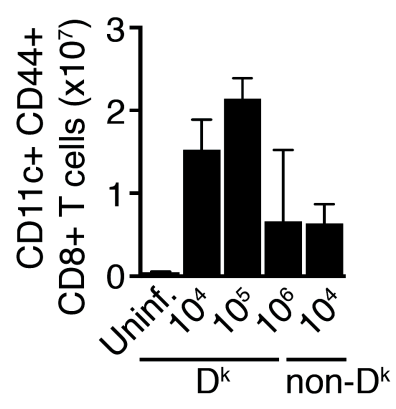

B

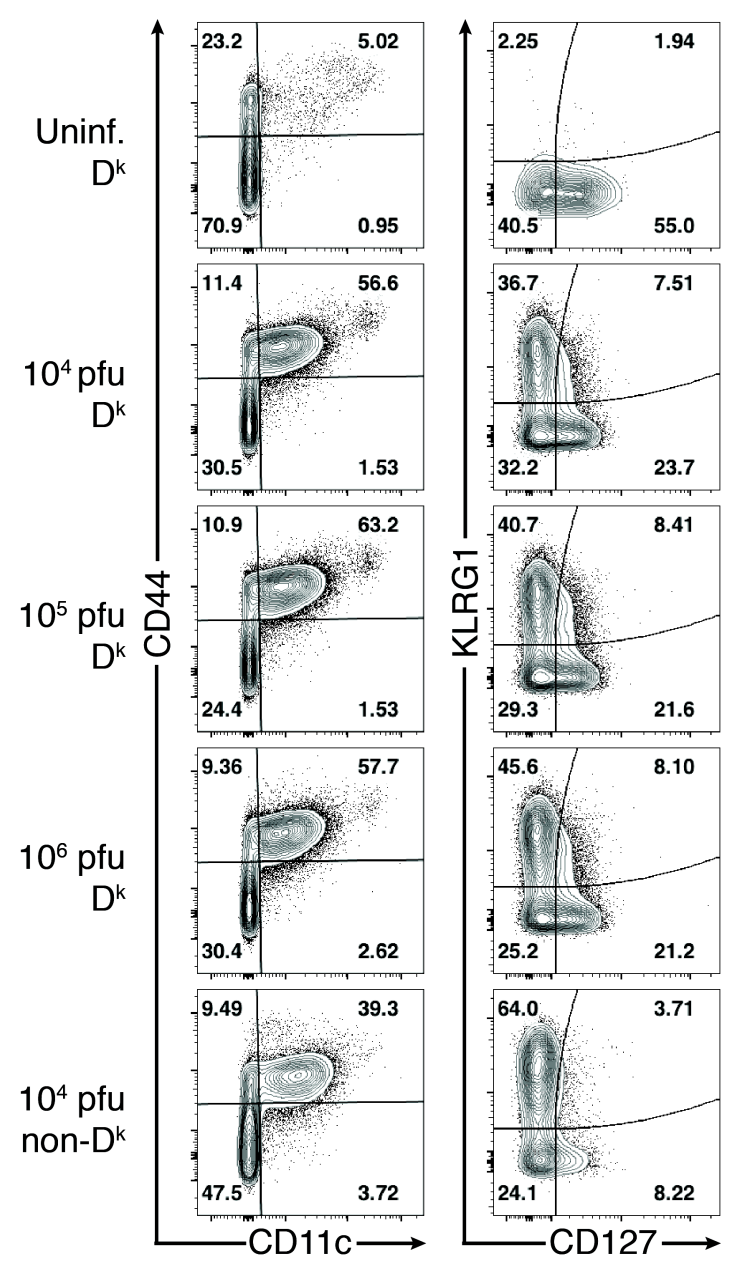

E

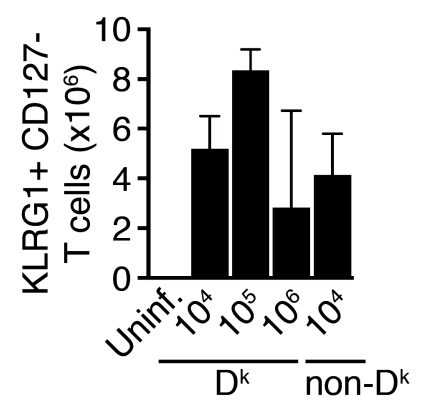

F

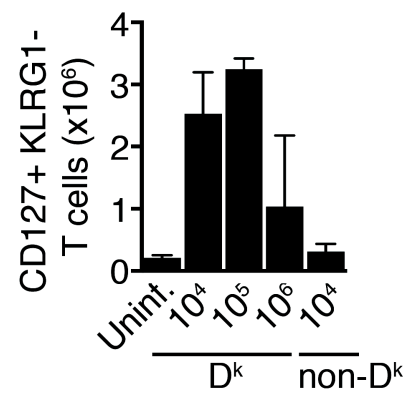

G

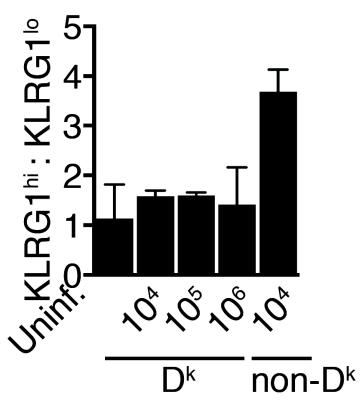


Figure 3.5. Licensed NK cell enhancement of CD8+ $T$ cell MPEC conversion is dependent on IL-10. Mice were treated with IL-10R blocking mAb (1B1.3A) or isotype control every other day during MCMV infection. Graphs display total numbers and/or frequencies $( \pm \mathrm{SD})$ of total $\mathrm{CD} 3+(\mathrm{A}), \mathrm{CD} 4+(\mathrm{B}), \mathrm{CD} 8+(\mathrm{C}), \mathrm{M} 45-\mathrm{D}^{\mathrm{b}}$-tetramer $+\mathrm{T}$ cells (D), M45-specific SLECs (F), and M45-specific MPECs (G). (E) Contour plots show the relative frequencies of M45-D ${ }^{\mathrm{b}}$-tetramer-gated SLECs (KLRG1+ CD127-) and MPECS (CD127+ KLRG1-). Data are representative of a single independent experiment ( $\mathrm{n}=4$ mice/group). Statistics were performed using one-way ANOVA $\left(* \mathrm{p}<.05,{ }^{*} \mathrm{p}<.01\right.$, $* * * \mathrm{p}<.001, * * * * \mathrm{p}<.0001)$. 
Figure 3.5
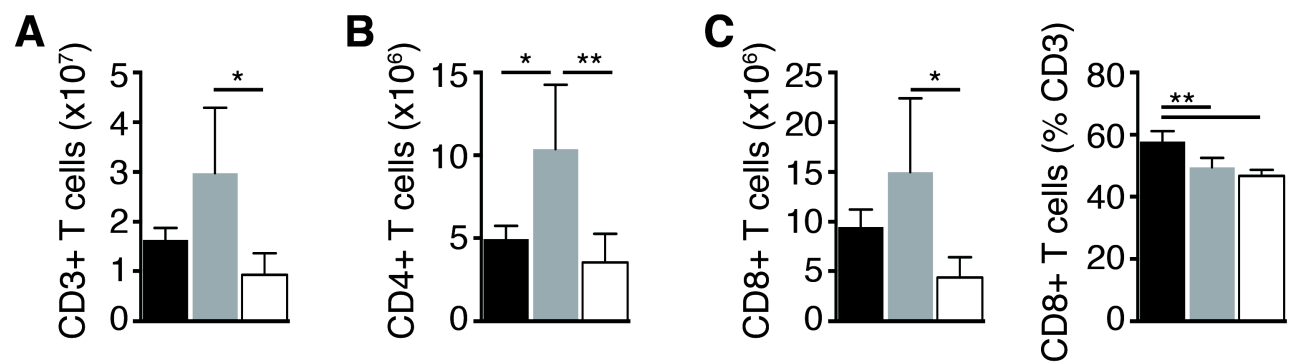

D

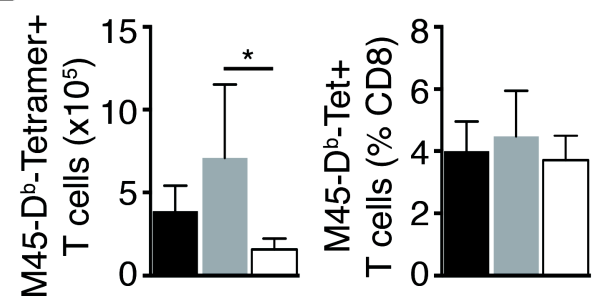

$$
\begin{aligned}
& -D^{k}+\text { Isotype } \\
& D^{k}+a-I L-10 R \\
& \text { Non- } D^{k}+\text { Isotype }
\end{aligned}
$$

E

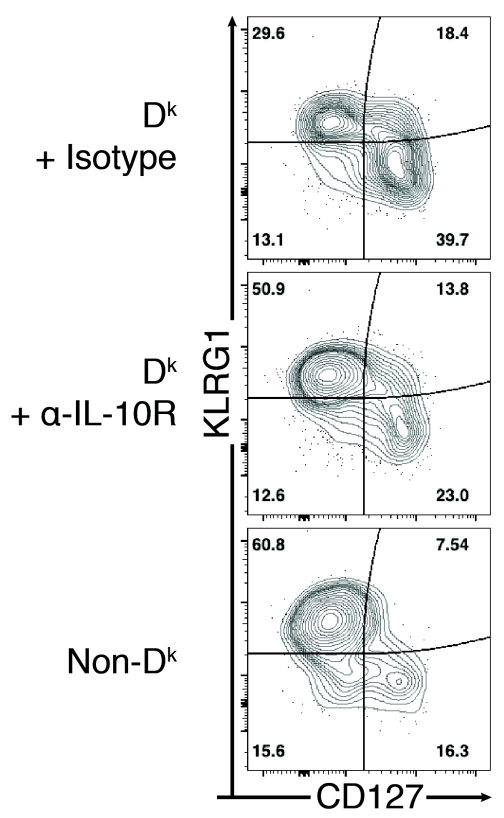

F

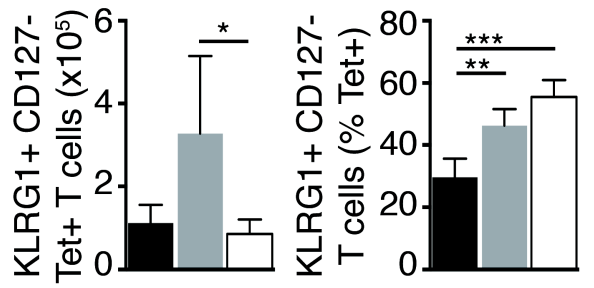

G

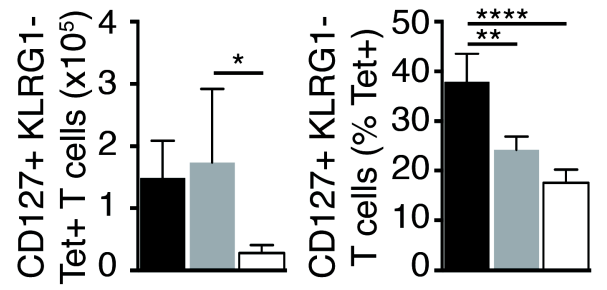


Figure 3.6. IL-10R signaling decreases DC maturation and the prevalence of patrolling monocytes. Mice were treated with IL-10R blocking mAb or isotype control every other day during MCMV infection for $8 \mathrm{~d}$. (A) Histogram plots display the surface expression of CD70 (left), CD86 (middle), and MHC II (right) for isotype-treated and IL10R-blocked mouse groups. Median fluorescent intensities for the indicated surface markers are reported. (B-D) Bar graphs display the mean frequencies $( \pm \mathrm{SD})$ of total $(B)$, CD70+ (C), and CD86+ DCs (D). (E) Contour plots show the relative frequencies of CD3- CD19- gated CD115+ CD11b+ monocytes (left) and monocyte-gated subsets (right). (F-I) Graphs show the mean frequencies $( \pm \mathrm{SD})$ of total monocytes $(\mathrm{F}), \mathrm{mPDCA}-$ $1+$ monocytes $(\mathrm{G}), \mathrm{CD} 11 \mathrm{c}-\mathrm{GR} 1^{\mathrm{lo}}$ patrolling monocytes $(\mathrm{H})$, and CD11c- GR $1^{\mathrm{int} / \mathrm{hi}}$ inflammatory monocytes (I). Data are representative of a single independent experiment ( $\mathrm{n}=4$ mice/group). Statistics were performed using one-way ANOVA $\left(* \mathrm{p}<.05,{ }^{*} \mathrm{p}<\right.$ $.01, * * * \mathrm{p}<.001, * * * * \mathrm{p}<.0001)$. 
Figure 3.6

A

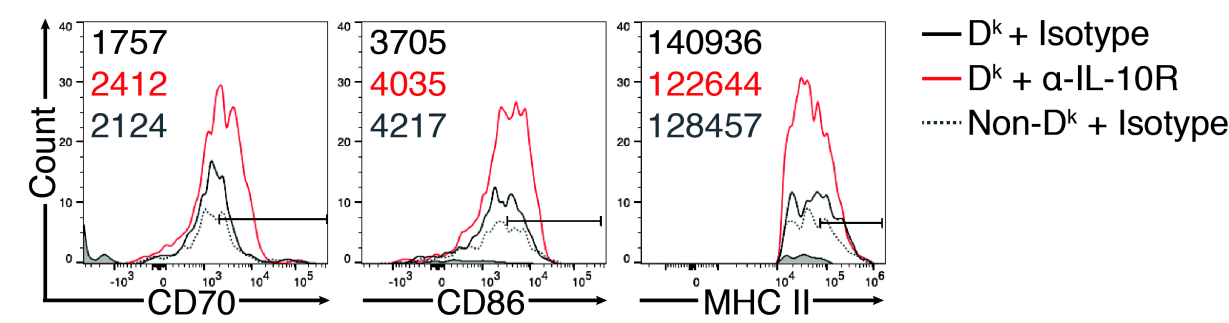

B

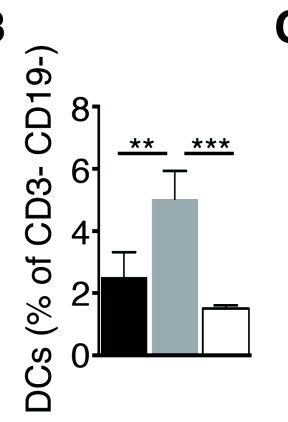

C

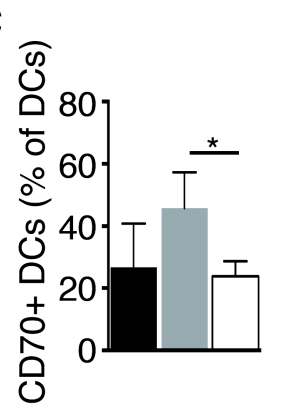

D

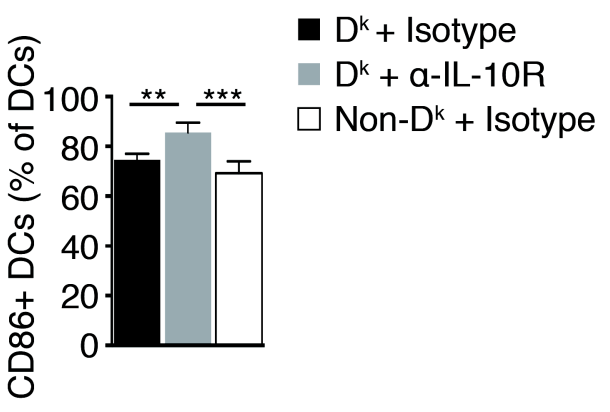

E
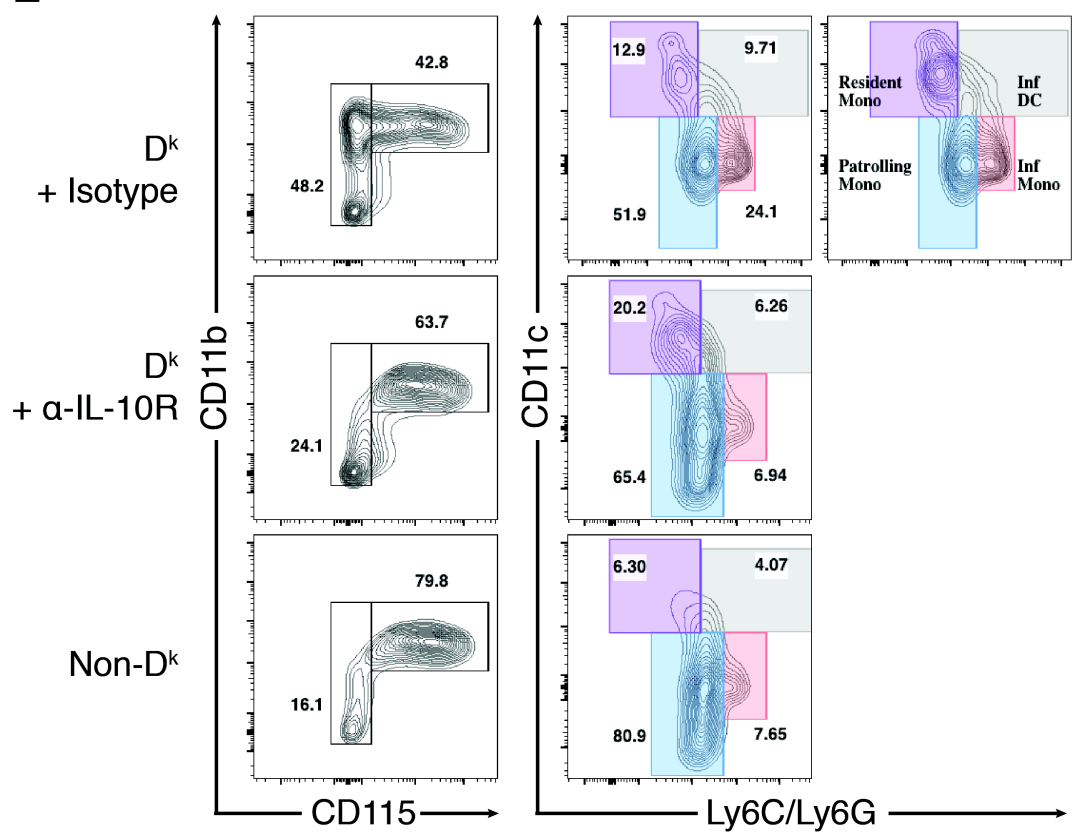

F

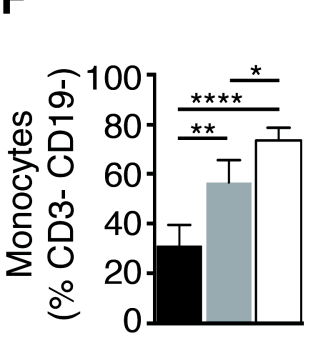

G

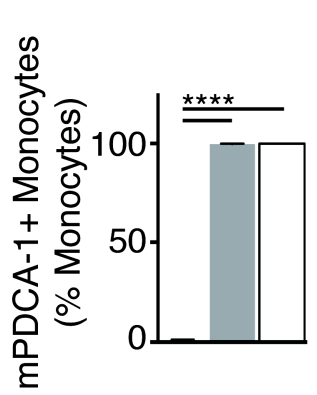

H

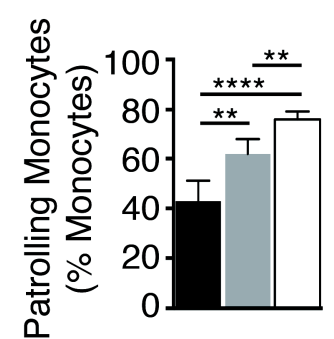

I

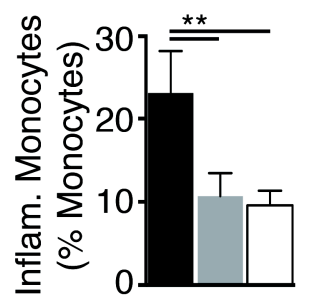




\section{Licensed $N K$ cell regulation of $C D 8+T$ cell differentiation is durable}

Although differentiated T cells were skewed towards MPECs in $\mathrm{D}^{\mathrm{k}}$ mice after acute MCMV infection, they were not fully mature memory cells (Figure 3.1, 3.3). Additionally, it was not clear if non- $\mathrm{D}^{\mathrm{k}}$ mice could establish a sizeable memory pool due to ongoing virus replication in the spleen through $\mathrm{d} 10$. Thus, we further investigated how NK cell-mediated skewing of T cell differentiation corresponds to CD8+ T cell phenotypes after viral control in the spleen. We first analyzed M45-specific CD8+ T cells in peripheral blood on $\mathrm{d} 20 . \mathrm{CD} 3+$ and $\mathrm{CD} 8+\mathrm{T}$ cell frequencies were comparable in naïve and infected cohorts regardless of the presence of $D^{\mathrm{k}}($ Fig. 3.7A,B). Moreover, the frequencies of M45-specific CD8+ T cells were similar for infected $D^{k}$ and non-D ${ }^{k}$ groups, and were significantly enriched relative to uninfected mice (Fig. 3.7C,D). However, closer examination revealed skewed $\mathrm{T}$ cell differentiation patterns similar to those already observed 8-10 d post-infection. While the frequency of M45-specific $\mathrm{CD} 127+\mathrm{CD} 8+\mathrm{T}$ cells in $\mathrm{D}^{\mathrm{k}}$ mice was nearly twice that of non- $\mathrm{D}^{\mathrm{k}}$ mice, a lack of $\mathrm{CD} 127+\mathrm{T}$ cells in non- $\mathrm{D}^{\mathrm{k}}$ mice was counterbalanced with an increased proportion of effector cells (Fig. 3.7C, E-G). Here, we distinguished central and effector memory CD8+ T cells by CD62L expression. In both groups, the frequency of effector memory ( $\mathrm{T}_{\mathrm{EM}}$; CD127+ CD62L-) in circulation was 2-4-fold greater than the frequency of circulating central memory cells $\left(\mathrm{T}_{\mathrm{CM}} ; \mathrm{CD} 127+\mathrm{CD} 62 \mathrm{~L}+\right)$. However, $\mathrm{D}^{\mathrm{k}}$ mice exhibited much greater frequencies of both $\mathrm{T}_{\mathrm{EM}}$ and $\mathrm{T}_{\mathrm{CM}}$ in comparison to non- $\mathrm{D}^{\mathrm{k}}$ mice, which followed from the heightened accumulation of MPECs during acute infection (Fig 3.7E,F). Therefore, the influence of $\mathrm{D}^{\mathrm{k}}$-licensed NK cells on early CD8+ T cell 
differentiation persisted beyond the acute infection when NK cells are known to be the most active. 
Figure 3.7. Licensed NK cell influence on $\mathbf{T}$ cell differentiation persists beyond acute infection. $\mathrm{D}^{\mathrm{k}}$-disparate mice were infected with $5 \times 10^{4}$ pfu MCMV i.p. On d 20, PBMCs were isolated and CD8+ T cells were examined. (A,B) Graphs represent the mean frequency $( \pm \mathrm{SD})$ of $\mathrm{CD} 3+(\mathrm{A})$ and $\mathrm{CD} 8+\mathrm{T}$ cells $(\mathrm{B})$ shown as proportions of live PBMCs. (C) Representative flow plots display live, CD3+ CD19-, CD8+ gated M45-D ${ }^{\text {- }}$ tetramer $+\mathrm{CD} 8+\mathrm{T}$ cells (top) and tetramer + gated CD127+CD62L $+\mathrm{T}$ central memory cells $\left(\mathrm{T}_{\mathrm{CM}}\right), \mathrm{CD} 127+\mathrm{CD} 62 \mathrm{~L}-\mathrm{T}$ effector memory cells $\left(\mathrm{T}_{\mathrm{EM}}\right)$, and CD127-CD62L- T effector cells $\left(\mathrm{T}_{\mathrm{Eff}}\right)$ (bottom). (D-G) Graphs represent the mean frequency $( \pm \mathrm{SD})$ of CD8+ gated M45- $\mathrm{D}^{\mathrm{b}}$-tetramer+ $\mathrm{T}$ cells $(\mathrm{D}), \mathrm{T}_{\mathrm{CM}}(\mathrm{E}), \mathrm{T}_{\mathrm{EM}}(\mathrm{F})$, and $\mathrm{T}_{\mathrm{Eff}}$ cells $(\mathrm{G})$. Data are representative of 3 independent experiments ( $n=4-8$ mice per group). Statistical analysis was performed using one-way ANOVA $(* \mathrm{P}<.05, * * \mathrm{P}<.01)$. 
Figure 3.7

A

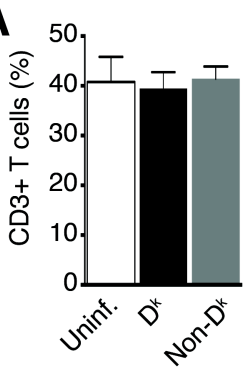

B

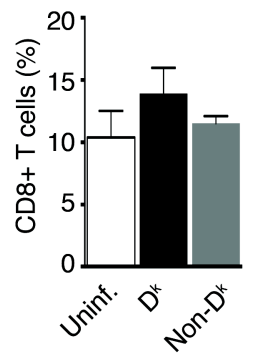

D

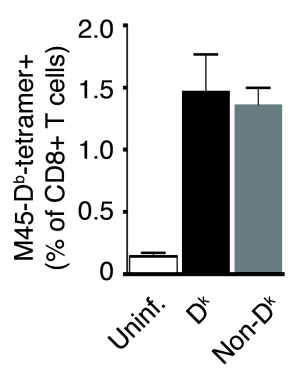

C

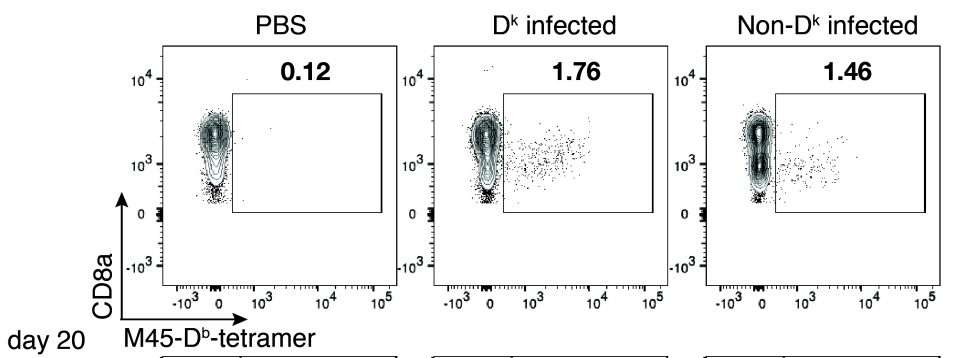

PBL

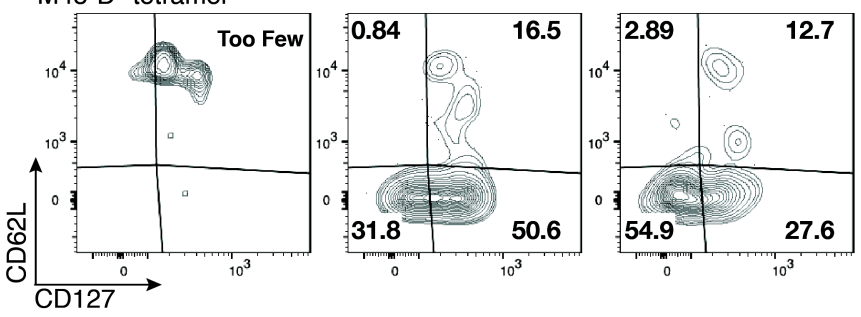

E

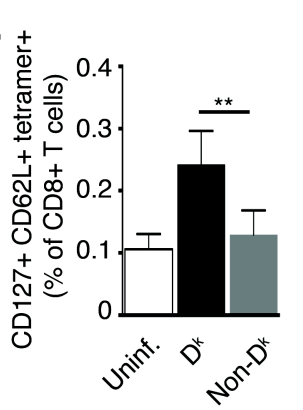

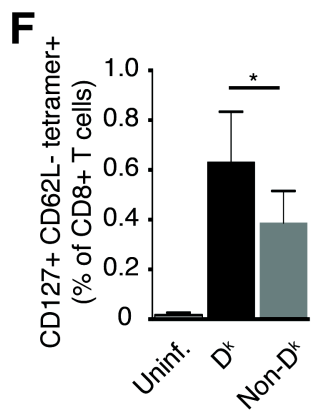

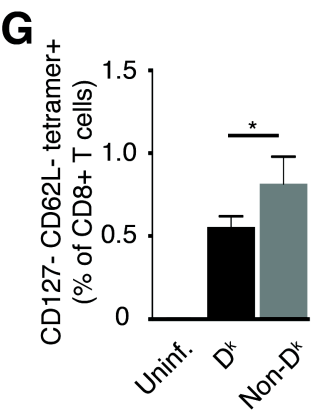




\section{Memory CD8+ $T$ cells primed in the presence of $D^{k}$-licensed NK cells remain elevated during viral latency}

Although CD8 $+\mathrm{T}$ cell phenotypes at d 20 corresponded with early differentiation profiles at 6-10 d post-infection (Figs. 3.1, 3.3, 3.7), it is possible that this reflected an active skewing process via persistent MCMV infection. Hence, increased viral dissemination or higher viral loads in non- $\mathrm{D}^{\mathrm{k}}$ mice might contribute to persistent induction of effector cells, which could mask memory T-cell detection. If so, one might expect skewed memory $\mathrm{T}$ cell phenotypes in the different strains to equilibrate once viral latency is established. To test this, we next analyzed T cells in $\mathrm{D}^{\mathrm{k}}$ and non- $\mathrm{D}^{\mathrm{k}}$ mice at 8 weeks post-infection, when MCMV latency is established in the salivary glands regardless of the efficiency of NK cell-mediated viral control (274). As expected, at d 56 MCMV was undetectable in spleen and liver tissues, while salivary gland virus was either below or very near the limit of detection, consistent with viral latency (Fig. 3.8A).

Although total splenic CD4+ and CD8+ T cell numbers were comparable in the different mice (Fig. 3.8B,C), we observed 2 to 3-fold higher numbers and percentages of M45-specific CD8+ T cells in $\mathrm{D}^{\mathrm{k}}$ mice compared to the non- $\mathrm{D}^{\mathrm{k}}$ group (Fig. 3.8D). At least half of the M45-specific CD8+ T cells in $\mathrm{D}^{\mathrm{k}}$ mice expressed CD127, and were 3times more abundant than memory $\mathrm{T}$ cells in non- $\mathrm{D}^{\mathrm{k}}$ mice $($ Fig. 3.8F). On the other hand, KLRG1+ effector CD8+ T cell numbers were comparable for both strains, despite that non- $\mathrm{D}^{\mathrm{k}}$ mice maintained proportionally more SLECs (Fig. 3.8E). Similar differences distinguished virus-specific $\mathrm{T}$ cell subset profiles obtained for $\mathrm{D}^{\mathrm{k}}$ mice with $\mathrm{G} 2+\mathrm{NK}$ cells intact or depleted during acute MCMV infection (Fig. 3.9A-C). Thus the impact(s) of licensed NK cells on T cell differentiation during acute MCMV infection is not only 
durable throughout persistent infection, but also regulates memory $\mathrm{T}$ cell formation during viral latency.

Although total and KLRG1+ antigen-experienced T cell numbers were similar in the different strains, we observed higher numbers of $\mathrm{CD} 127+$ antigen-experienced $\mathrm{T}$ cells in $\mathrm{D}^{\mathrm{k}}$ mice, consistent with skewed profiles observed for M45-specific T cells (Fig. 3.8G). Similar accrual of inflationary CD $8+T$ cells amongst both groups of mice at $\mathrm{d} 56$ might have accounted for the reduced distinction in total virus-responsive $\mathrm{T}$ cell numbers, as inflationary memory cells expand after acute infection has been resolved $(203,209)$. Consequently, the effect(s) of licensed NK cell-mediated virus resistance may have less of a pronounced impact on inflationary $\mathrm{T}$ cell accumulation. In support of this possibility, both $\mathrm{D}^{\mathrm{k}}$ and non- $\mathrm{D}^{\mathrm{k}}$ mice exhibited equal numbers of cytokine-responsive CD8+ T cells upon re-stimulation with either non-inflationary (M45, m139) or inflationary (m139, M38, IE3) MCMV peptides (Fig. 3.8 H,I).

Together, these data demonstrate that highly specific licensed NK cell control of MCMV accelerates accumulation of $\mathrm{CD} 8+\mathrm{T}$ cells and biases $\mathrm{T}$ cell differentiation towards MPECs over terminal SLEC fates, which endures throughout persistent and latent viral infection. Moreover, the ability to limit viral persistence in the salivary glands is not compromised in mice with efficient licensed NK cell control. Likewise, the accrual of both inflationary and non-inflationary effector CD8+ T cells remains intact in both $D^{k}$ and non- $D^{k}$ mice (Fig. 3.8). 
Figure 3.8. Efficient licensed NK cell-mediated MCMV resistance corresponds with improved central and effector memory $\mathrm{CD8}+\mathrm{T}$ cell maintenance during viral latency. $\mathrm{D}^{\mathrm{k}}$-disparate mice were infected with $5 \times 10^{4} \mathrm{pfu}$ MCMV. (A) Viral levels were measured in spleen, liver, and salivary gland tissues (d 56). (B,C) Graphs display the mean number $( \pm \mathrm{SD})$ of splenic CD4+ $(\mathrm{B})$ and CD8+ T cells $(C)$. (D-F) Shown are the total numbers and frequencies $( \pm \mathrm{SD})$ of M45-specific CD8+ T cells $(D)$, SLECs $(E)$ and CD127+ memory cells (F). (G) Bar graphs depict total numbers ( \pm SD) of CD44+ CD11c+ virus-experienced CD8+ T cells, SLECs (middle), and memory cells (right). $(\mathrm{H}, \mathrm{I})$ Graphs display the mean number $( \pm \mathrm{SD})$ of $\mathrm{IFN} \gamma+(\mathrm{H}, \mathrm{left}), \mathrm{TNF} \alpha+(\mathrm{H}$, right $)$, and CD107a + CD8 + T cells (I) upon in vitro peptide re-stimulation with non-inflationary (M45, m139) and inflationary MCMV peptides (m139, M38, IE3). Data are representative of $5(A-G)$ or $2(H-I)$ independent experiments ( $n=3-4$ mice/ group). Statistics were calculated using one-way ANOVA or unpaired Student's t-tests $(* \mathrm{P}<.05$, $* * \mathrm{P}<.01)$. 
Figure 3.8

A
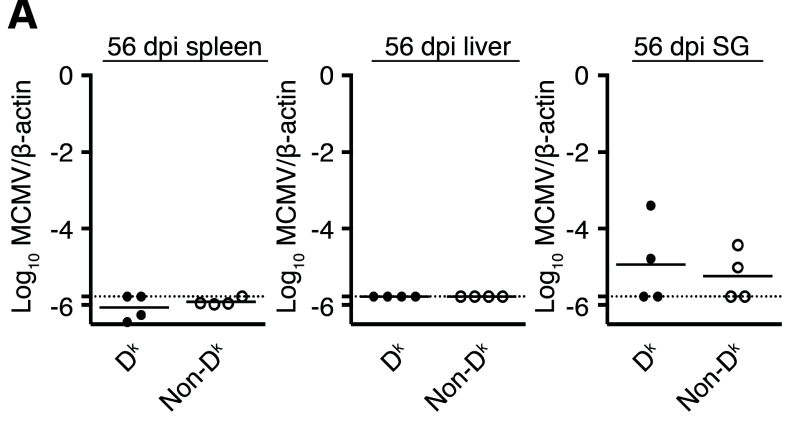

B

C
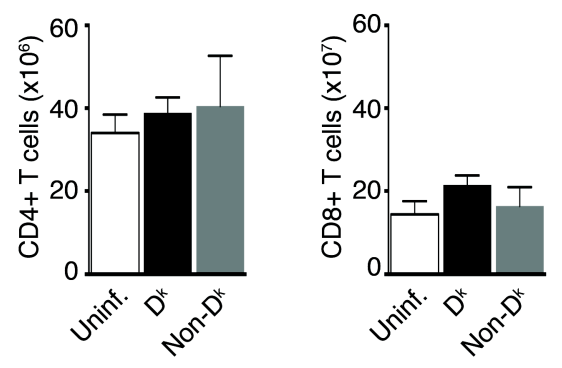

D
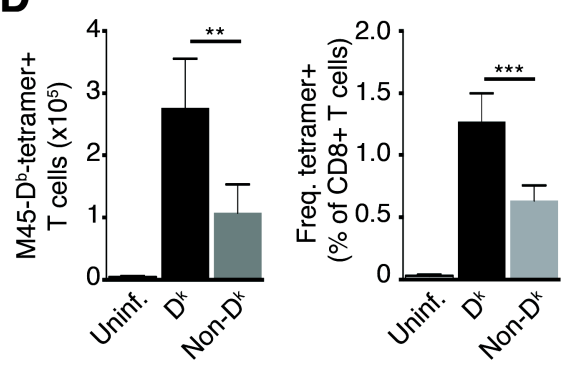

F
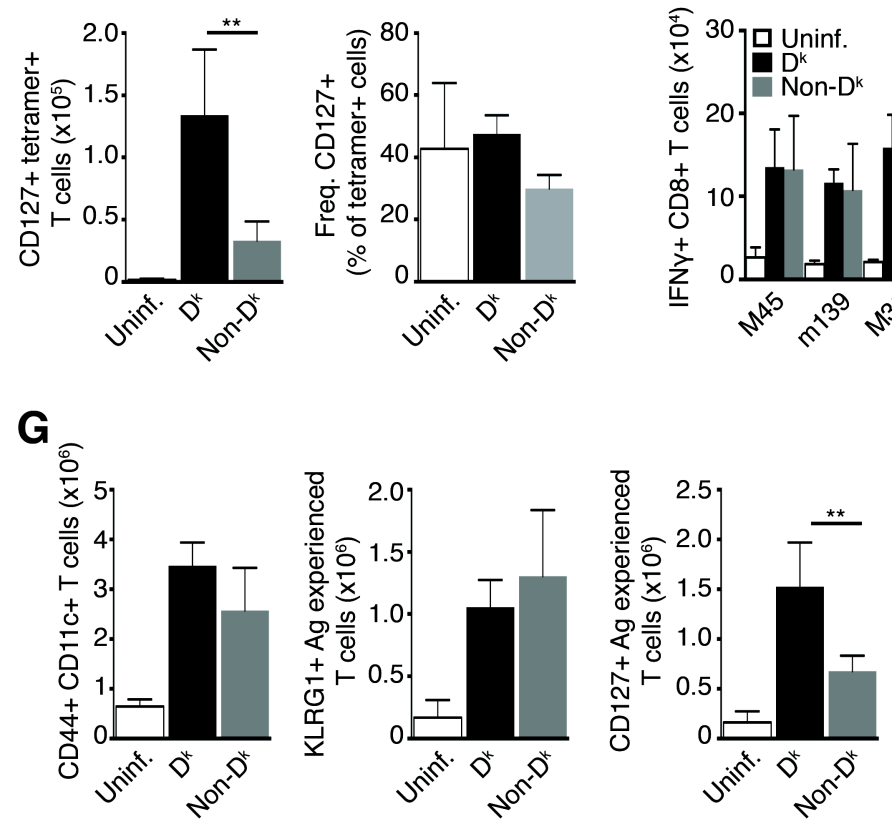

E

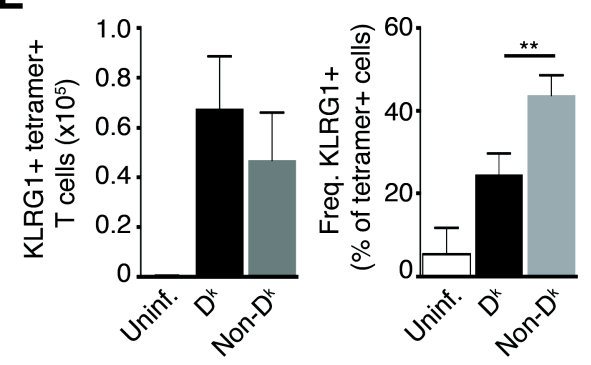

H
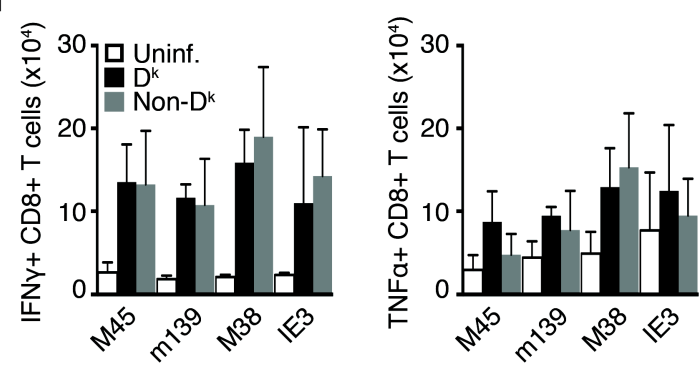

I

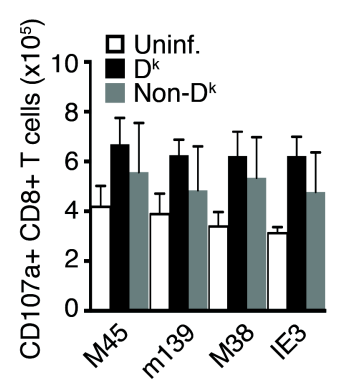


Figure 3.9. MHC I $D^{\mathrm{k}}$-licensed G2+ NK cell responses to acute MCMV imprint on long term CD8+ $\mathbf{T}$ cell differentiation skewing. $\mathrm{D}^{\mathrm{k}}$ mice were treated with $\mathrm{G} 2+\mathrm{NK}$ cell-depleting mAb or isotype control, and were subsequently infected with $5 \times 10^{4} \mathrm{pfu}$ MCMV for 56 d. (A-C) Graphs represent the mean ( \pm SD) frequency of splenic CD8+ T cells (A), antigen experienced CD8+ T cells (B), and SLECs (left), and CD127+ CD27+ memory cells (right) (C). Data are representative of at least 3 independent experiments (n $=3-4$ mice/group $(* \mathrm{P}<.05, * * \mathrm{P}<.01$ by unpaired Student's t-test). 
Figure 3.9
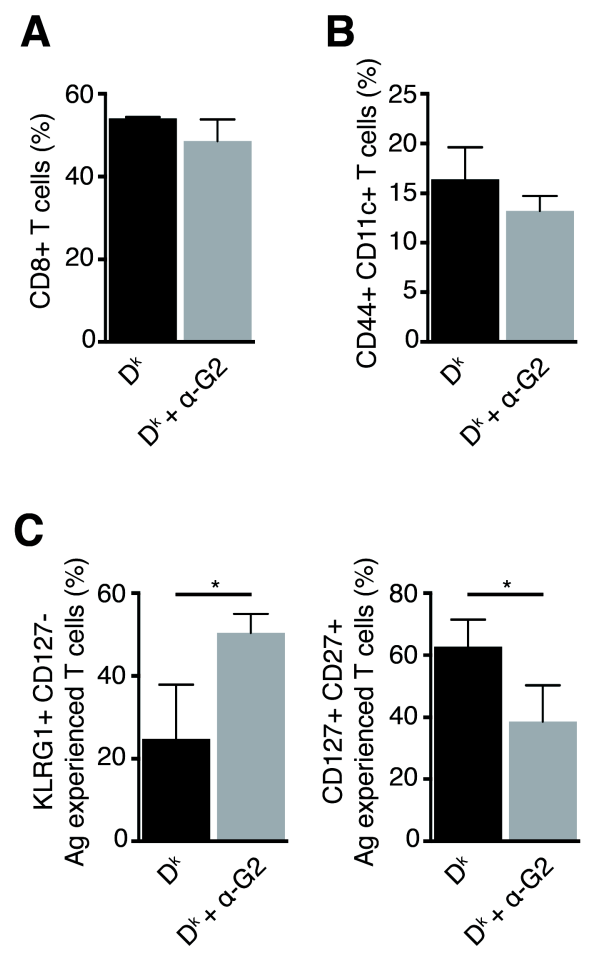


\section{Licensed NK cells control CD8+ memory $T$ cell immunity to secondary viral challenge}

Increased virus-specific memory CD8 $+\mathrm{T}$ cells in $\mathrm{D}^{\mathrm{k}}$ mice suggested that these $\mathrm{T}$ cells primed in $\mathrm{D}^{\mathrm{k}}$ mice might also confer improved immunity to secondary viral challenge. However, due to the presence of neutralizing antibodies (275), systemic antigen stimulation of SLECs (208), and the potential for latent virus reactivation (276), re-infecting hosts would not allow us to specifically interrogate the contributions of memory CD8+ T cells . Hence, we adoptively transferred CD8+ T cells from latently infected (d 56) $\mathrm{D}^{\mathrm{k}}, \mathrm{G} 2+\mathrm{NK}$ cell depleted $\mathrm{D}^{\mathrm{k}}$, and non- $\mathrm{D}^{\mathrm{k}}$ mouse groups into naïve, $\mathrm{G} 2+$ NK cell-depleted and MHC-matched hosts. At the time of transfer, donor CD8+ T cells were phenotypically skewed as seen previously (Fig. 3.8, Fig. 3.9). Recipient mice were immediately challenged with high dose MCMV after cell transfer (Fig. 3.10A). Significant body weight-loss was observed amongst all groups, though recipients of virus-experienced $\mathrm{T}$ cells began recovering body weight by $\mathrm{d} 3$, whereas non-transfer recipients lost weight throughout the infection (Fig. 3.10B, left). Interestingly, recipients of $\mathrm{CD} 8+\mathrm{T}$ cells primed in either non- $\mathrm{D}^{\mathrm{k}}$ or $\mathrm{G} 2+\mathrm{NK}$ cell depleted mice controlled virus more efficiently than recipients of $\mathrm{T}$ cells primed in the presence of $\mathrm{D}^{\mathrm{k}}$-licensed $\mathrm{G} 2+\mathrm{NK}$ cells (Fig. 3.10B, right). Protective immunity and viral control in this condition therefore corresponded to higher frequencies of effector cells present in both non- $\mathrm{D}^{\mathrm{k}}$ and G2-depleted $D^{k}$ mice at the time of cell transfer. Hence, these results suggested that effector cells might remain poised to mediate efficient viral control after adoptive transfer and followed immediately by secondary viral challenge. 
To pursue this and determine whether CD8+ memory $\mathrm{T}$ cells continue to persist sans antigen, we again transferred donor $\mathrm{T}$ cells primed in the presence or absence of licensed G2+ NK cells during acute MCMV infection into MHC matched recipients. However, secondary MCMV challenge was delayed by one week (Fig. 3.10C). Interestingly, despite that $\mathrm{T}$ cells primed without $\mathrm{G} 2+\mathrm{NK}$ cells displayed greater frequencies of effector cells upon donor cell transfer, KLRG1+ CD127- CD8+ T cells comprised less than $4 \%$ of the antigen-experienced population in both recipient groups by d 5 post-transfer (data not shown). Upon secondary virus challenge, body weight recovery was improved in recipients of experienced donor CD8+ T cells, regardless of the priming condition (Fig. 3.10D, left). Moreover, CD8+ T cells primed in G2-replete donors controlled MCMV in spleen and liver tissues to nearly undetectable levels at d 5 post-infection (Fig. 3.10D, right). Although $\mathrm{T}$ cells primed in G2+ NK cell-depleted donors also mediated significant viral control relative to the non-transfer recipients, virus levels were nonetheless higher. These data demonstrate that CD8+ T cells primed together with highly efficient licensed NK cell control of MCMV infection were competent to provide efficient immune protection against secondary viral infection. As varied degrees of viral protection were observed depending on the nature of the challenge, this might reflect distinct roles for the skewed subsets of virus-specific CD8 $+\mathrm{T}$ cells primed in disparate hosts. Of course, despite the skewed proportions of memory cells and SLECs in the transferred CD8+ T cells, hosts replete with G2+ NK cells during acute infection sustained similar numbers of cytokine producing SLECs as non- $\mathrm{D}^{\mathrm{k}}$ and G2+ NK cell depleted groups (Fig. 3.8). Therefore, mice with highly specific licensed-NK cell control of MCMV maintain multiple mechanisms for immediate virus- 
specific responses (NK cells, KLRG1+ SLECs), and can maintain a quiescent reserve of central memory cells. In contrast, mice with less efficient NK cell control of infection skew T cell differentiation to elicit a sizeable pool of virus-specific SLECs that are poised for immediate response. 
Figure 3.10. CD8+ T cells transferred from mice without acute licensed NK cell control confer improved viral control to secondary challenge. (A,C) Schematics for the transfer of $3 \times 10^{6}(\mathrm{~A})$ or $5 \times 10^{6}(\mathrm{C})$ splenic CD8+ T cells from primary MCMVinfected mouse strains into naïve MHC-I matched and G2+ NK cell-depleted recipients. Transfer recipients were infected with $1 \times 10^{5}$ pfu MCMV within $2 \mathrm{~h}$ post-transfer (A) or $2.5 \times 10^{5}$ pfu MCMV 1 week post-transfer $(C)$. (B,D) Shown are the mean $( \pm$ SD) changes in body weight for the indicated recipient groups (left) and d 5 virus levels in spleen and liver tissues (right). Data are representative of 2-3 independent experiments ( $n=4-6$ mice/group). Statistics were calculated using one-way or two-way ANOVA $(* \mathrm{P}<.05$, $* * \mathrm{P}<.01, * * * \mathrm{P}<.001, * * * * \mathrm{P}<.0001)$ 
Figure 3.10

A

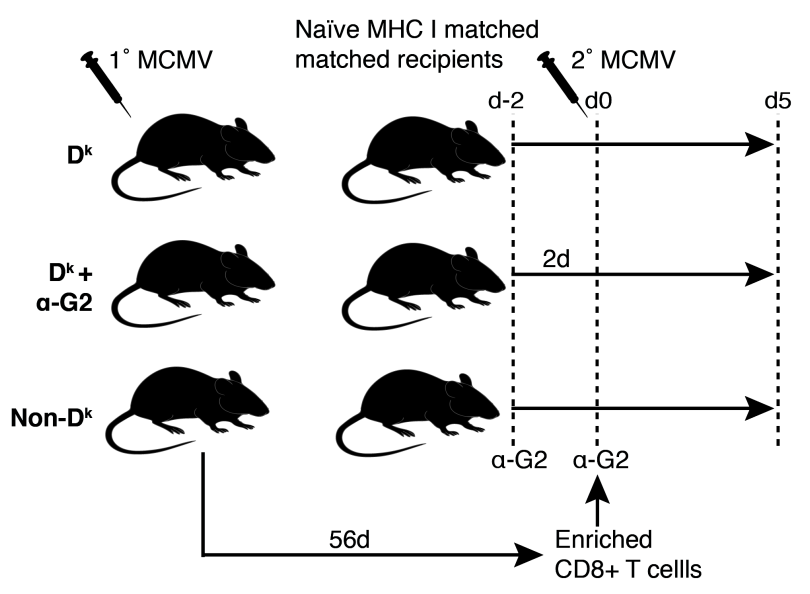

B

$3 \times 10^{6} \mathrm{CD} 8+\mathrm{T}$ cells transferred

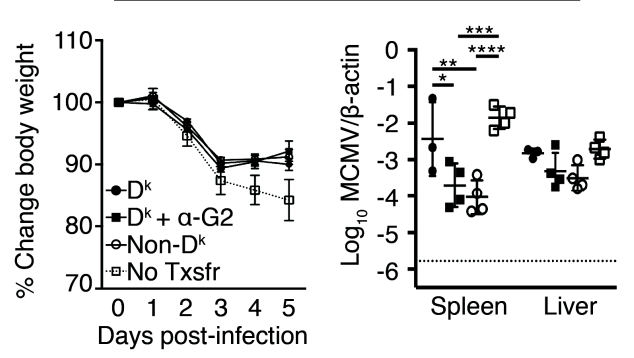

C

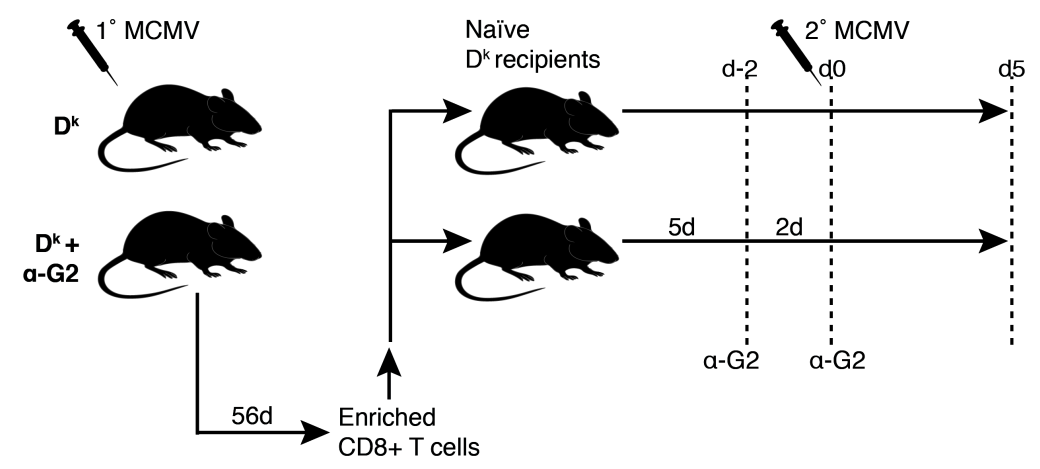

D $5 \times 10^{6} \mathrm{CD} 8+\mathrm{T}$ cells transferred

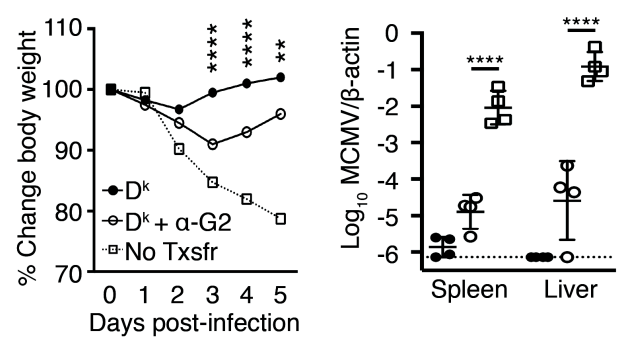




\section{Discussion}

It is well established that innate immune cell sensing and recognition of pathogen and disease-state signatures (e.g. PAMPS, stress-inducible ligands, modification of MHC-I) not only invokes natural immunity, but also instructs the magnitude, quality, and type of adaptive immune responses generated (8). NK cells are potent innate lymphoid cells that provide early resistance to viral infection and several varieties of cancer (277). The last two decades have revealed key discoveries about how genetically encoded NK receptors and MHC-I haplotypes influence the mechanisms through which NK cells discern healthy self from target cells (56). Moreover, these mechanisms of NK-mediated resistance can have far-reaching impacts on the host adaptive immune response (11). While several studies have investigated the impacts of cytokine activated or viral ligandactivated NK cells on adaptive immunity, the influence of specific antiviral responses by MHC I licensed-NK cells has remained largely underexplored.

Here we examined the impacts of licensed-NK cell responses to MCMV infection on $\mathrm{CD} 8+\mathrm{T}$ cell differentiation, maintenance, and memory recall. Comparing MHC Idisparate mice with or without $\mathrm{D}^{\mathrm{k}}$-licensed NK cells, we discovered that efficient licensed NK cell-mediated viral control biases CD8+ T cell differentiation towards MPECs over SLECs within the first 10 days of infection in an IL-10 dependent manner. This early impact on differentiation skewing was resilient, and indeed contributed to enhanced CD8+ T cell memory maintenance throughout viral latency. Moreover, this skewing impacted the quality and type of CD8+ T cell immunity provided upon secondary viral challenge (Fig. 3.11). 
Figure 3.11. Model of licensed NK cell influence on memory CD8+ T cell differentiation and distinct $T$ cell responses to secondary challenge. (A) Efficient $D^{k}$ licensed G2+ NK cells reduce early pro-inflammatory cytokine responses, and limit the spread and duration of viral infection in the spleen. This corresponds with an earlier accumulation of virus-specific CD8+ T cells. T cells are predominantly SLECs at the peak of T cell accrual (d 6), but the proportion of MPECs increases upon T cell contraction (d 8-10). Non- $\mathrm{D}^{\mathrm{k}}$ mice and G2+ NK cell-depleted mice do not control MCMV efficiently, which results in increased pro-inflammatory cytokines, prolonged DC maturation, and sustained viral infection in the spleen. This corresponds with delayed CD8+ T cell accumulation (d 8), and is chiefly composed of SLECs. Upon T cell contraction, minimal MPEC accumulation is observed. In both groups, T cell differentiation at d 10 is durable through 20 and $56 \mathrm{~d}$ post-infection. (B) Absolute numbers of $\mathrm{T}_{\mathrm{Eff}}$ are equivalent for both groups in the presence of antigen (left). In contrast, greater numbers and proportions of $\mathrm{T}_{\mathrm{CM}}$ cells are observed in mice with licensed G2+ NK cells (right). Whereas both groups are protected against secondary viral challenge, the increased presence of $\mathrm{T}_{\mathrm{CM}}$ confers improved resistance in the absence of persistent antigen. 
Figure 3.11

A
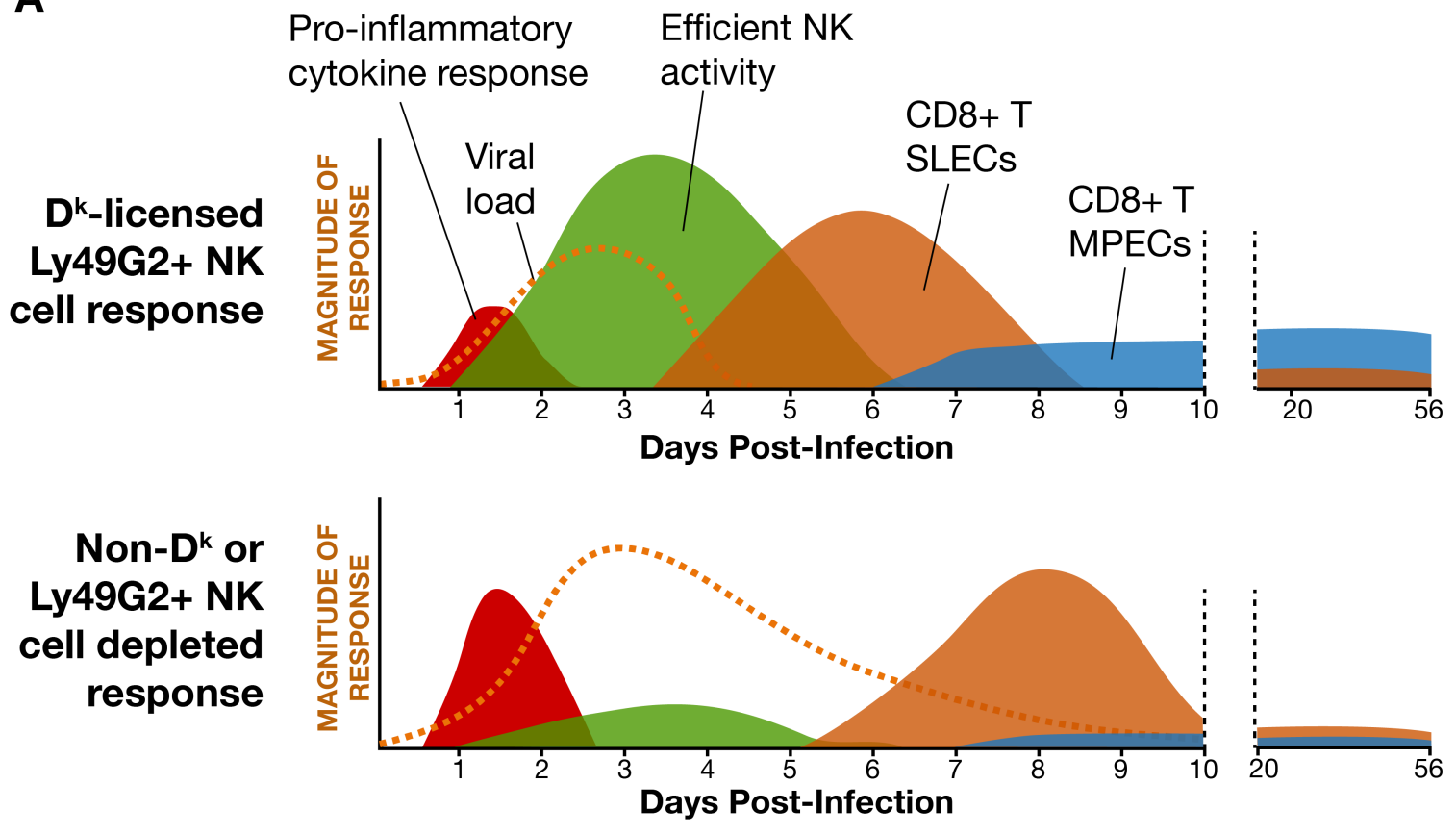

B

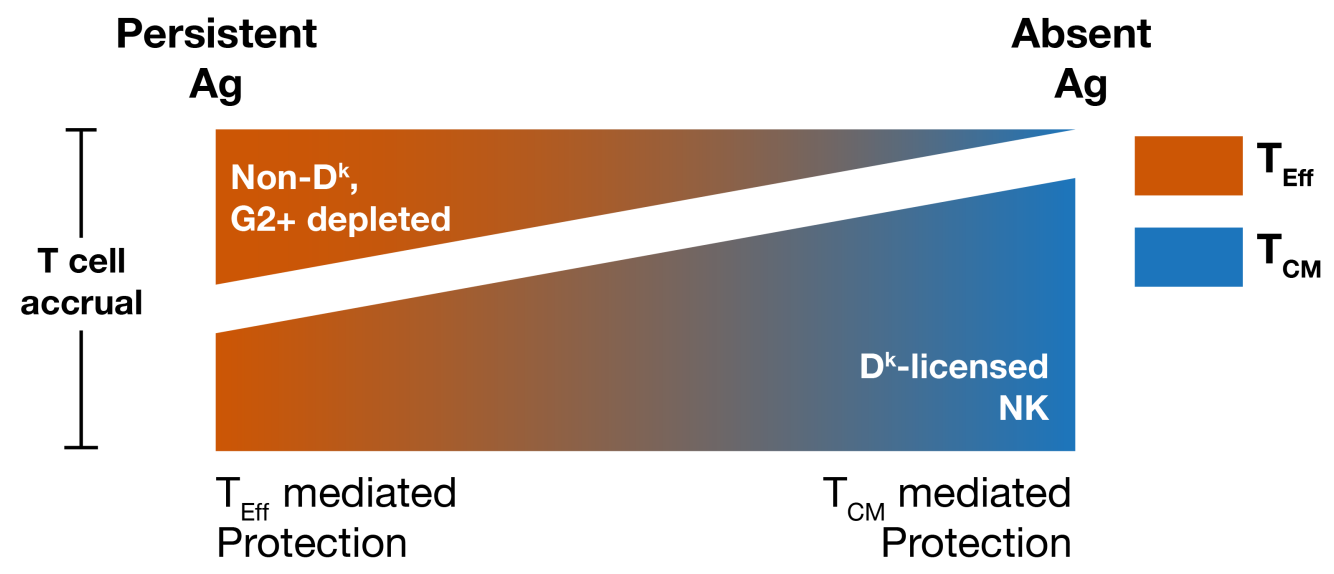


Notably, our results indicate that the influence of $\mathrm{D}^{\mathrm{k}}$-licensed NK cells on CD8+ T cell immunity is distinct from that of Ly49H+ NK cells. Both mechanisms of NK cell recognition and response to MCMV confer viral control within $3.5 \mathrm{~d}(47,71,78)$. Likewise, efficient viral resistance in through either mechanism corresponds with enhanced recovery of dendritic cells and improved CD8 $+\mathrm{T}$ cell accumulation during acute MCMV infection $(23,24)$. However, despite this early support for CD8+ T cell immunity, mice with Ly49H+ NK cell-mediated control exhibit a reduced accumulation of virus-specific $\mathrm{CD} 4+$ and $\mathrm{CD} 8+\mathrm{T}$ cells after acute infection compared to more susceptible mouse strains $(106,109)$. In contrast to this, the present study revealed that mice with highly specific $\mathrm{D}^{\mathrm{k}}$-licensed NK cell responses to MCMV elicit a 3-fold greater number of memory CD8+ T cells in, relative to mice without $\mathrm{D}^{\mathrm{k}}$-licensed $\mathrm{G} 2+\mathrm{NK}$ cells. This disconnect between $\mathrm{Ly} 49 \mathrm{H}+$ and licensed-G2+ NK responses to infection and their impacts on CD8 $+\mathrm{T}$ cell immunity suggests that efficient viral control in and of itself is not a chief determinant of CD8 $+\mathrm{T}$ cell memory formation. Rather, these differences indicate that distinct mechanisms of NK cell-mediated viral resistance may govern how NK cells interact with adaptive immune cells.

Ly49H NK subsets confer critical resistance via efficient recognition of the m157ligand (47-49) and are more efficient at mediating viral resistance when unimpeded by licensing/inhibitory receptors that can interact with their cognate class-I $(82,83)$. However, rapid elimination of infected host cells, including infected antigen-presenting cells such as DCs $(107,109)$, may limit the abundance of antigen for $\mathrm{T}$ cell priming. Indeed, mature infected DCs are capable of priming CD8 $+\mathrm{T}$ cells (278), and may be critical to initiating adaptive immune responses early after infection. In contrast, 
homeostatic interactions between the inhibitory G2 receptor and self-MHC I ligands enhances the ability of G2+ NK cells to mediate critical viral resistance $(68,71,78)$. Although the precise activating stimuli underlying $\mathrm{D}^{\mathrm{k}}$-licensed NK recognition of MCMV infected targets in vivo have yet to be determined, the dominant responding NK cell subset during infection is nonetheless strongly inhibited by cells with normal MHC-I expression.

During viral infection and in an inflammatory environment, NK killing of adaptive immune cells and DCs can be moderated by increased MHC-I expression. Recent studies have highlighted that NK cells can lyse activated CD4+ and CD8+ T cells during certain viral infections $(13-17,223)$. Moreover, leukocyte resistance to NKmediated lysis is regulated by type I IFN induced expression of MHC-I and downregulation of NCR1- and NKG2D-ligands $(16,17,228)$. Since $\mathrm{D}^{\mathrm{k}}$-licensed NK cells mediate efficient viral control without restricting adaptive immunity, it is possible that clearance of infected DCs and activated CD8 $+\mathrm{T}$ cells is inhibited by MHC I interactions with G2. Still another possibility for the enhanced maintenance of CD8+ T cells and skewed memory differentiation is the licensed NK cell impact on co-stimulatory ligand display. Previously, we discovered that splenic DC expression of CD70 and CD86 is efficiently down regulated during MCMV infection in $\mathrm{D}^{\mathrm{k}}$ mice, whereas non- $\mathrm{D}^{\mathrm{k}}$ mice sustained expression for an extended period [Teoh et al. Manuscript accepted. J Immunol (2016)]. Moreover, CD8 $+\mathrm{T}$ cell responses in mice without licensed NK cell control of MCMV proceeded independent of CD70-CD27 interactions, suggesting that additional inflammatory mediators and prolonged antigen stimulation were capable of driving robust effector cell responses. In the present study, we demonstrated that enhanced 
memory differentiation in $\mathrm{D}^{\mathrm{k}}$ mice was dependent on IL-10 signaling. IL-10 signaling was required to reduce terminal $\mathrm{CD} 8+\mathrm{T}$ effector cell differentiation and interference with IL-10 signaling recapitulated inflammatory phenotypes observed in mice without specific licensed NK control. Kaech and colleagues (270) demonstrated similar requirements for IL-10 to shape memory precursor effector cells during LCMV infection. In that study, regulatory T cells produced IL-10 to reduce the maturation of splenic DCs and lessen inflammatory cytokines upon T cell contraction. We infer similar mechanisms of IL-10 guided memory precursor formation during MCMV infection, though further studies are warranted to determine the source and kinetics of IL-10 production.

Despite these significant differences in memory $\mathrm{T}$ cell priming and differentiation, $C D 8+T$ cells primed with or without $\mathrm{D}^{\mathrm{k}}$-licensed $\mathrm{NK}$ cells present were capable of conferring vital resistance to secondary MCMV infection. CD8+ T cells primed in the absence of $\mathrm{D}^{\mathrm{k}}$-licensed G2+ NK cells bestowed enhanced immunity to naïve recipients when challenged immediately after adoptive cell transfer. We infer that the SLEC-skewed donor T cells in this setting were poised for effector activity, and that the immediate viral stimulus sustained their primed effector activities. On the other hand, CD8 $+\mathrm{T}$ cells primed in the presence of licensed G2+ NK cells also conferred substantial viral control in naive mouse recipients, but only when the secondary virus challenge was delayed after cell transfer. This requisite delay may suggest that trafficking to lymph nodes and/or secondary lymphoid organs is necessary for these memory-skewed cells to mediate optimal protective immune functions (158). Thus distinct roles for CD8+ T cells were shaped by the quality of early NK cell responses to acute infection, and these roles 
likely reflect host specific needs for immediate immune responses that track inversely with the efficiency of NK responses.

The present study offers unique insights into host specific NK cell immunity, and how early distinctions in NK responses to infection impact the early differentiation fate of $\mathrm{CD} 8+\mathrm{T}$ cells. Moreover, these data provide the groundwork to further investigate whether NK cells inform CD8 $+\mathrm{T}$ cell differentiation at a single, critical juncture or whether distinct differentiation pathways are continuously curated by host-specific NK cell responses. 


\section{Chapter IV}

General Discussion and Future Directions 


\section{Introduction}

Traditional hallmarks of innate and adaptive immunity such as immune cell response kinetics and sensitization requirements continue to separate these major arms of cellular immunity. However, recent studies have uncovered significant overlapping functions and characteristics for these two compartments, particularly for NK and CD8+ T cells. For example, NK cells have been demonstrated to form trained memory-like responses (279), and in some cases even exhibit hapten- or antigen-specific recall responses $(126,280)$. In the same vein, memory CD8+ T cells have been shown to harbor specific molecular signatures similar to innate cells, altering homeostatic levels of signaling molecules levels such as STAT4 in order to provide immediate effector responses independent of TCR-restricted antigen (281). Defining new and diverse functional roles for $\mathrm{NK}$ and $\mathrm{CD} 8+\mathrm{T}$ cell subsets has also uncovered novel cellular interactions and regulatory pathways between both cell types. Because of this interplay, NK cells are an attractive target for therapeutic intervention, particularly for chronic infections such as HIV and HCV, which are typified by exhausted CD8+ T effector cell activity (282). However, studies in mice and humans have demonstrated varied impacts of NK cells on CD8 $+\mathrm{T}$ cells across a range of genetically diverse animal models and infections (11). This suggests that host- and disease-specific elements influence the basis of these cellular interactions.

MHC I molecules are critical determinants of NK cell surveillance specificities and responsiveness to infection. Inhibitory receptor interactions with host-MHC at rest serve to both inhibit NK effector activity against healthy cells and to tune NK cells to respond efficiently to damaged or diseased tissues (54). Although inhibitory NK 
receptors can dampen NK cell responses to certain viral infections in specific hosts, particularly when direct NK receptor recognition of a viral ligand is involved $(82,83)$, it is also appreciated that strongly licensed NK cells are essential for mediating improved graft versus leukemia responses after HSCT as well as antiviral protection against a variety of viral pathogens (54). Moreover, it is now demonstrated that the early activities of NK cells during viral infection shape inflammatory responses and ultimately direct CD8 $+\mathrm{T}$ cell differentiation. However despite these new findings and identification of key players, much remains to be clarified with regard to the specific mechanisms and determinants that guide NK cell influences on CD8+ T cells. Furthermore, it will be of great interest to better understand how these influences on CD8+ T-cell differentiation will ultimately shape long-term host immunity, not only in the context of a secondary or reactivated viral challenge, but also in the context of bystander protection against a bevy of viral pathogens.

\section{Licensed NK cell responses to viral infection influence the quality and duration of signals 1-3 for priming $\mathrm{CD8}+\mathrm{T}$ cell responses}

\section{Induction of alternative co-stimulatory and inflammatory pathways}

Several research groups, including our own, have correlated the efficiency of NK cell-mediated immune responses with the kinetics and magnitude of CD8+ T-cell accumulation and effector function $(22-24,106,109,261,283)$. Notably, this effect of $\mathrm{NK}$ cells is often relayed to the CD8+ T cells indirectly via manipulation of selective DC survival and maturation; however direct regulation of CD8+ T cell subsets has also been 
demonstrated $(13,15-18,223)$. In a prior study of licensed NK cell-enhanced CD8+ T cell immunity, we suggested that licensed NK cells qualitatively influenced the priming potential of DCs during MCMV infection- versus a purely quantitative recovery of antigen presenting cells (24).

We hypothesized that DCs in mice with licensed NK cells can preferentially exhibit co-stimulatory ligands needed to enhance CD8+ T cell immunity. However, the present work demonstrated that the kinetics of splenic DC maturation - as defined by expression of CD86, CD70, and MHC II - were similar in the presence and absence of $\mathrm{D}^{\mathrm{k}}$-licensed G2+ NK cells. Moreover, CD70 and CD86 were downregulated earlier in mice with efficient control of infection (Fig. 2.8). Despite having a prolonged expression of $\mathrm{CD} 70$ and $\mathrm{CD} 86$, we found that mice without $\mathrm{D}^{\mathrm{k}}$ or $\mathrm{G} 2+\mathrm{NK}$ cells were capable of priming primary CD8 $+\mathrm{T}$ cell responses independent of CD70 (Figure 2.9). The precise mechanism(s) by which CD8 $+\mathrm{T}$ cells are primed in mice without licensed NK cell control remains a major area of interest, particularly as this may lead to distinctions in the cues that guide memory differentiation (Chapter III). It is possible that alternative costimulatory pathways and/or cytokine profiles contributed to these CD70-independent CD8 $+\mathrm{T}$ cell responses, particularly since matched splenic viral levels were not sufficient to recapitulate dominant SLEC skewing in $\mathrm{D}^{\mathrm{k}}$ mice at $\mathrm{d} 8$ (Fig. 3.4).

The necessity of multiple co-stimulatory pathways to accommodate contextspecific immune responses has been previously demonstrated for a variety of viral infections, infectious doses, and even across spatio-temporal kinetics of a single viral infection $(146,153,259)$. Additional unbiased screens of splenic DC surface molecules and molecular signatures are needed in order to identify inflammatory and co-stimulatory 
pathways that may aid CD70-independent MCMV-specific CD8+ T cell responses in mice without efficient licensed NK cell control. Comparative microarray or RNAsequencing for sorted splenic DC populations from $\mathrm{D}^{\mathrm{k}}$ mice treated with and without $\mathrm{G} 2$ depleting antibodies will help to detect prominent differences in the transcription of costimulatory genes. Isolation of splenic DCs from both cohorts at various times after infection may be necessary to define critical kinetic differences in co-stimulatory ligand display. Moreover, refined sorting of predefined maturation subgroups (CD86+ CD70+ $\mathrm{MHCII}^{\mathrm{hi}}$ ) may help to clarify whether distinctions in gene expression are due to differences in individual maturation profiles amongst cohorts or due to the varied ratios of mature to immature DCs in each cohort.

While we expect DC transcriptional signatures to distinct $\mathrm{D}^{\mathrm{k}}$ mice from $\mathrm{G} 2+\mathrm{NK}$ depleted mice as a result of varied resistance to MCMV, of particular interest would be the gene transcripts ontologically related to immune responses and co-stimulation. Croft and colleagues have previously demonstrated roles for OX40 and 4-1BB in shaping CD8+ T cell responses to acute and persistent MCMV infection $(150,151)$. OX40 stimulation boosted primary antiviral CD8 $+\mathrm{T}$ cell responses to MCMV independent of CD4+ T cells, though CD4+ T cells were necessary to promote OX40-dependent enhancement of persistent CD8+ T cell responses (151). Similarly, 4-1BBL-independent stimulation of $4-1 \mathrm{BB}$ at the time of initial priming - perhaps via extracellular matrix proteins, such as laminin-1 (284) - dampened primary CD8+ T cell accumulation; however, 4-1BB/4-1BBL interactions during acute infection were critical for the maintenance of memory CD8+ T cells during persistent and latent phases of MCMV (150). Differences in 4-1BBL expression and/or other non-classical 4-1BB ligands would 
be of considerable interest given the bi-phasic impact of 4-1BB on CD8+ T cell priming and persistence and the partial resemblance to phenotypes observed for mice with or without specific licensed NK cell control. Still, additional co-stimulatory family members may also contribute to the differential shaping of CD8+ T cell immunity (e.g. LIGHT, GITR) (Fig. 4.1), any of which could then be explored through appropriate knockout models or transient blocking studies, similar to the present investigation of CD70/CD27 interactions (Chapter II).

In addition to classical co-stimulatory pathways, we would also investigate differences in the relative copy number of cytokine transcripts. Once again, while we anticipate variable cytokine transcript profiles from both DC cohorts, we would take special interest in cytokines previously implicated in CD8+ T cell differentiation. Comparisons of transcript levels for Il12(p35), Il15, Il10, Ifna, and Ifnbl would be particularly informative, as these signal 3 cytokines have previously been identified as key determinants of CD8+ T cell fate $(133,156,269,270)$. Although the abundance of cytokine transcripts does not necessarily correlate with cytokine concentrations, these data would nonetheless inform our interpretations of potential pathways that modify DC stimulation of CD8 + T cells in mice with and without G2+ NK cells, especially for comparisons performed over a time course. Verification of specific cytokine production and analysis of cytokine concentrations could then be performed through serum sampling and quantification via multiplex immunoassays or conventional ELISA.

Of course, in addition to targeted interests, unbiased molecular screens would likely reveal new and unexpected differences in DC subsets from the described cohorts, including, but not limited to cell adhesion molecules, cell metabolism, pathogen sensing, 
and survival. Such discoveries may provide new and important avenues for exploring how the efficiency of licensed NK cell responses to MCMV shape DC priming potential, and consequently, the $\mathrm{CD} 8+\mathrm{T}$ cell repertoire. 
Figure 4.1. Highly specific licensed NK cell responses to MCMV influence CD8+ T cell priming through multiple pathways. The quality and efficiency of NK cell responses to viral infection guide primary $\mathrm{CD} 8+\mathrm{T}$ cell reliance on specific costimulatory and inflammatory cues. Distinct NK cell responses to infection could potentially impact $\mathrm{T}$ cell priming through three non-exclusive pathways. (i) Direct NKDC interactions might induce specific co-stimulatory ligands/receptors on DCs depending on the manner in which NK cells respond to infection. (ii) Licensed G2+ NK cells could stimulate unique cytokine signatures, or modify the abundance of inflammatory cytokines produced. Likewise, IL-10 production by NK cells or alternative cell sources in the wake of efficient NK cell control might dampen DC maturation upon resolution of infection. (iii) The efficiency of NK responses to viral infection can also impact the duration of viral replication and the corresponding abundance of antigen for TCR-p:MHC I interactions. The dose of antigen might override certain T cell requirements for co-stimulatory signaling, and could also influence $\mathrm{T}$ cell differentiation. 
Figure 4.1

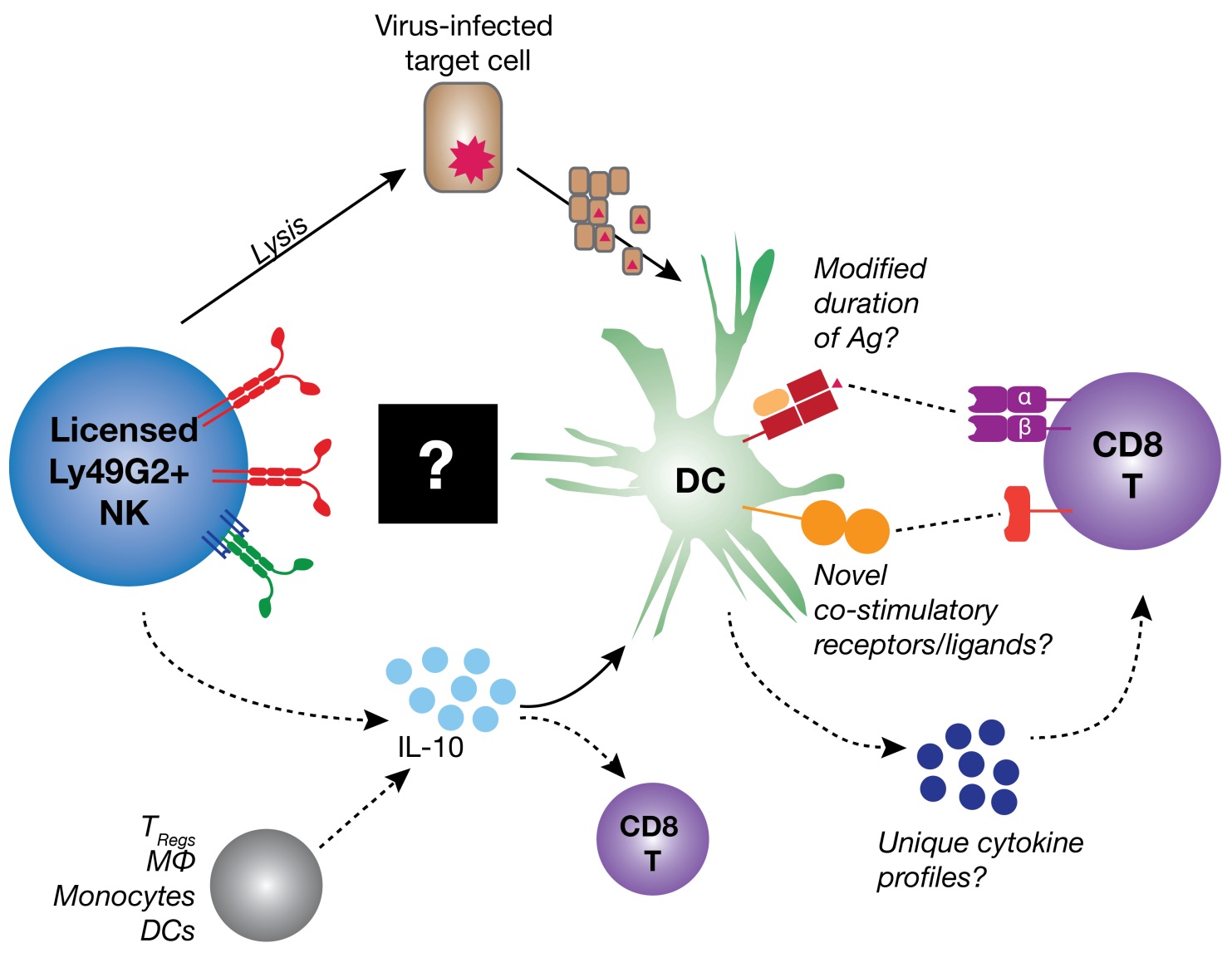

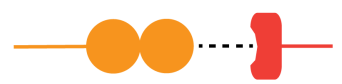

Costimulatory ligand/ receptor candidates

OX40L / OX40

4-1BBL / 4-1BB

GITRL / GITR

LIGHT / HVEM

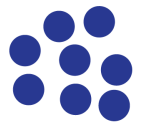

Cytokines of interest

IL-12

IL-15

IL-10

IFNa/ $\beta$ 


\section{Extended duration of antigen exposure}

In addition to influencing the expression of specific co-stimulatory pathways and cytokine profiles, NK cells can also directly inform the extent of active viral replication and the availability of antigen for priming $\mathrm{T}$ cells (Fig. 4.1). As demonstrated in

Chapters II and III, the NK cell responses to certain viral infection can determine the magnitude and duration of acute viral levels, and this undoubtedly impacts the extent and chronicity of $\mathrm{T}$ cell exposure to specific viral antigen. In a model of mouse adapted influenza (PR8) NK cells were demonstrated to be critical for targeting infected cells in the lung and indirectly providing cell debris for DC uptake and antigen presentation to T cells in the draining lymph nodes (93). However, it has also been proposed that highly specific NK responses that clear viral infection too hastily may diminish total antigen availability and reduce the magnitude of $\mathrm{CD} 8+\mathrm{T}$ cell responses to infection, as observed in specific mouse strains during MCMV infection $(106,109)$. Thus the context and manner in which NK cells respond to certain viral infections can wield significant influence over the quality and duration of antigen presentation.

Although it is generally agreed that signals 2 and 3 (costimulation and cytokines, respectively) are required to support optimal effector and memory functions of $\mathrm{T}$ cells, TCR-peptide-MHC (TCR-p:MHC) interactions (signal 1) that meet a critical response threshold have been demonstrated to elicit effector responses in the absence of additional co-stimulation (285-287). Early studies of this phenomenon focused solely on the contribution of CD28 through use of CD28-deficient mice, CTLA-4-Ig transgenic mice, or neutralizing antibodies to CD80/86 ligands - none of which precluded the potential contributions of alternative co-stimulatory pathways (286-289). Frehlinger and 
colleagues have since recapitulated these findings using cell-free, peptide-tetramer priming assays to rule out the contribution of alternative or unidentified co-stimulatory interactions (285). The response threshold for signal 1 is set by both the affinity of TCRp:MHC interactions as well as the overall density of peptide presented, which is a product of both antigen concentration as well as chronicity of antigen display $(290,291)$. These parameters are at least partially redundant, such that increasing either the density or affinity of specific antigens can effectively promote $\mathrm{CD} 4+$ and $\mathrm{CD} 8+\mathrm{T}$ cell responses (291, 292). With that said, CD8+ T cells do discriminate between the potency and density of the antigen: Whereas a high density of peptides can compensate for relatively low affinity TCR interactions and prompt $\mathrm{T}$ cell proliferation, the affinity of TCRp:MHC interactions, regardless of density, specifically informs certain functional properties, such as IL-2 production $(292,293)$.

While many of the abovementioned studies were performed in vitro or carried out using synthetic peptides sans infection, studies examining priming of anti-viral CD8+ T cell responses in the absence of specific co-stimulatory networks have also been performed. Early investigations of LCMV infection in either CD28 deficient or CTLA-4Ig transgenic mice revealed that despite yielding lower neutralizing antibody titers, these mice mounted robust cytotoxic $\mathrm{CD} 8+\mathrm{T}$ cell responses to infected target cells and delayed-type hypersensitivity reactions to LCMV footpad injections $(286,289)$. Interestingly, whereas highly replicative viruses such as LCMV or VV prompted robust $\mathrm{T}$ cell responses in the absence of CD28 co-stimulation, less virulent or low replicating viral infections mounted poor or absent cytotoxic lymphocyte responses without CD28 (289). Additional studies examining requirements for CD27 or OX40 with viruses of 
modified virulence or persistence have demonstrated similar findings, that less replicative and less chronic pathogens require greater amounts of co-stimulation $(152,258)$. While redundancies in co-stimulatory pathways or cytokine signaling can compensate for specific deficiencies in the co-stimulatory molecules examined (149), the impact of antigen abundance and persistence is nonetheless an important factor to consider.

In the present work, we demonstrated that mice with efficient $\mathrm{D}^{\mathrm{k}}$-licensed NK cell control of MCMV primed early CD70/CD27-dependent CD8+ T cell responses, whereas mice without specific licensed NK cell control exhibited a delayed but CD27independent primary $\mathrm{CD} 8+\mathrm{T}$ cell response (Fig. 2.4, Fig. 2.9). Moreover, we observed a preferential skewing of CD8 $+\mathrm{T}$ cells towards MPECs or SLECs in mice with and without $\mathrm{D}^{\mathrm{k}}$, respectively (Fig. 2.10, Fig. 3.1). Differences in the duration or class of costimulatory ligand expression likely contributed to this skewing, as prolonged expression of CD86 and CD70 corresponded with enhanced SLEC skewing (Fig. 2.6). Remarkably, however, these kinetic phenotypes also recapitulated in vitro $\mathrm{T}$ cell proliferation in response to low and high doses of antigen (288). In that study, low doses of antigen promoted early CD8 $+\mathrm{T}$ cell responses that were entirely dependent on CD28 signaling, whereas high doses of antigen demonstrated robust but delayed proliferation in the absence of CD80 and CD86. Thus, it is possible that the increased presence of virus, and presumably viral antigen, in non- $\mathrm{D}^{\mathrm{k}}$ mice was sufficient to prime $\mathrm{CD} 27$-independent $\mathrm{CD} 8+\mathrm{T}$ cells in response to MCMV. However, since differences in splenic viral levels do not directly indicate differences in antigen abundance, this hypothesis warrants further investigation. 
To interrogate the impact of antigen availability independent of active viral replication, exogenous non-virus associated antigen could be introduced to mice with and without $\mathrm{D}^{\mathrm{k}}$ during MCMV infection, essentially exploiting the viral infection as an adjuvant. By dissociating the antigen bolus from replicative virus, the impact of antigen quality and quantity could be examined while maintaining the environmental priming differences in mice with and without specific licensed NK cell control of MCMV. OVA

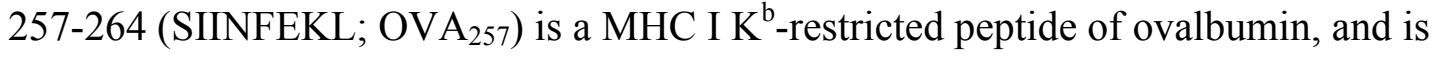
commonly used as a strong immunogen to elicit $\mathrm{OVA}_{257}$-specific CD8+ T cell responses (294). Utilizing the defined $\mathrm{OVA}_{257}$ peptide rather than the whole ovalbumin protein would allow us to control for potential differences in host protein processing. Likewise, TCR-transgenic $\mathrm{T}$ cells that specifically recognize $\mathrm{OVA}_{257}(\mathrm{OT}-\mathrm{I})$ have been used to track clonal $\mathrm{T}$ cell responses to antigen stimulation (295). Thus, we could transfer congenically marked OT-I T cells into recipient mice prior to MCMV infection to normalize starting frequencies of antigen-specific T cells. Antigen could then be introduced to experimental groups in one of two ways: (i) Soluble OVA 257 peptide could be directly introduced to the mice intravenously in predefined doses and regimens. In this setting, the foreign peptides would function similar to viral antigen released from infected cells, and could be engulfed by DCs for presentation to CD8+ T cells, as described previously (296). One caveat to this approach, however, could result from differences in antigen uptake by DCs in MHC-disparate mice during MCMV infection. To investigate this possibility, we could conjugate fluorescent molecules to $\mathrm{OVA}_{257}$ prior to administration and interrogate total and subset-specific DC uptake of the fluorophorelabeled antigen (296). While differential uptake by total or specific subsets of DCs 
would invalidate use of this assay, these results would nonetheless inform our understanding of the quality and kinetics of antigen uptake by DCs in MHC-disparate hosts.

(ii) An alternative approach to examining the impact of specific antigen doses on T cell priming would involve the transfer of defined numbers of $\mathrm{OVA}_{257}$-pulsed DCs into the mice, as described previously (297). This approach would approximate equal DC presentation of the peptides, at least upon initial administration. However, while levels of antigen presentation may be better controlled, the maturation of DCs could potentially be more artificial, as generation of DCs for immunization typically requires in vivo expansion with Flt3-ligand expressing tumor cells (B16-FLT3L) or ex vivo treatment with GM-CSF to derive bone-marrow derived DCs. Moreover, DCs are typically matured with LPS prior to peptide pulsing, which could mask the influence of NK cells on DC-T cell interactions. Despite this, artificial maturation would be relatively equal for DCs delivered to both experimental groups. By comparing OT-I T cell responses relative to endogenous MCMV-specific T cells we could assess the general influence of antigen dose and persistence in $\mathrm{D}^{\mathrm{k}}$-disparate strains.

\section{Specific lymphoid compartment impacts on T cell priming}

With regard to how $\mathrm{T}$ cells interact with $\mathrm{p}: \mathrm{MHC}$ and co-stimulatory molecules during MCMV infection, we must acknowledge that to this point, we have predominantly focused on $\mathrm{T}$ cell interactions with DCs in the spleen. Combined with the liver, the spleen is one of the primary sites of acute MCMV infection, and where early NK cell responses are most active $(298,103)$. Investigating the spleen offers many advantages 
for examining the impact of licensed NK cells on T cell priming. (i) As a primary site of viral amplification, a surplus of viral antigen is readily accessible in the spleen and can prompt rapid recruitment, proliferation and response of primed T cells. (ii) Stromal cells lining the splenic marginal zones are early cell targets of infection $(10,103)$. Moreover, DCs and NK cells both localize in close proximity to infected stromal cells at the marginal zone between 48-96 hours post-infection, which could provide a platform for direct NK-DC-T cell interactions $(10,94,103)$. (iii) Since NK cells can influence the efficiency of viral control in the spleen, distinct inflammatory environments can manifest and impact the manner in which $\mathrm{T}$ cells are initially primed.

Early priming of non-inflationary CD $8+\mathrm{T}$ cells in peripheral lymph nodes has been relatively underexplored for MCMV infection [Non-hematopoietic cells in the inguinal lymph nodes have previously been demonstrated to present MCMV antigen and drive inflationary, but not non-inflationary, memory CD8+ T cell responses after infection (299)]. While tissue-specific environments could foster differential T cell priming conditions, this is not always the case. Indeed, splenic-priming of CD8+ T cells during a mouse-adapted influenza infection (PR8; i.n.) was found to stimulate effector and memory CD8 $+\mathrm{T}$ cells that were equally as functional and protective as $\mathrm{T}$ cells primed in the lung draining lymph nodes, despite a lack of active viral replication in the spleen (300). Interestingly, when MCMV is introduced to the host systemically (i.p.), the virus first traffics from the peritoneal cavity to the mediastinal lymph nodes to gain access to the bloodstream where it can disseminate to peripheral tissues as free virus (103). Moreover, infection of CD169+ macrophages in the subcapsular sinus of the mediastinal lymph nodes demonstrates that virus could be retained in the lymph node for 
antigen processing and presentation. Temporal components of $\mathrm{T}$ cell priming in distinct organs, such as the duration or frequency of TCR-p:MHC/co-stimulatory interactions can substantially impact the quality of antiviral CD8 $+\mathrm{T}$ cell responses, as evidenced by comparisons of in vivo and in vitro priming systems (290). Thus, it would be of great interest to examine whether $\mathrm{T}$ cell accumulation and differentiation in the lymph node differs between MHC-disparate mouse strains as observed in the spleen.

To investigate organ specific priming effects on CD8 $+\mathrm{T}$ cells we could employ immunomodulatory reagents that block entry and egress of CD8+ T cells from the lymph nodes. FTY720 is a sphingosine-1-phosphate receptor-1 (S1P(1)) agonist that downregulates $\mathrm{S} 1 \mathrm{P}(1)$ on the surface of $\mathrm{CD} 8+\mathrm{T}$ cells and sequesters $\mathrm{CD} 8+\mathrm{T}$ cells in the lymph nodes (301). FTY720 would allow us to effectively examine the profile of T cells primed in the lymph node by sequestering $\mathrm{CD} 8+\mathrm{T}$ cells and preventing their egress to peripheral sites of infection. Similarly, treatment with MEL-14 ( $\alpha$-L-selectin) mAb prevents lymphocyte entry into lymph nodes via high endothelial venules (302). This Lselectin neutralizing antibody would enable us to prevent entry of CD8+ T cells that were primed in the spleen into the lymph node. As described previously, congenically marked OT-I T cells could be transferred to MHC-disparate host recipients prior to systemic infection in order to control for the starting frequencies of naïve clonal cells. We could then utilize MCMV that has been modified to express $\mathrm{OVA}_{257}$ (MCMV-OVA) in order to stimulate OT-I cells with virus-derived antigen. Following the transfer - after the requisite amount of time for cells to circulate and populate lymphatic organs has passed and the infection with MCMV-OVA, treatment with FTY720 and MEL-14 would halt inter-lymphatic trafficking. We could then survey lymph node-primed CD8+ T cells at 
various periods post-infection and compare OT-I expansion and differentiation to splenic populations by standard flow cytometric analysis. We would be particularly interested to monitor T cell differentiation, as local inflammatory environments in the lymph node should be similar between strains early after MCMV infection. Furthermore, since T cells would be sequestered in the lymph nodes, we could also pre-label transferred OT-I cells with a fluorescent dye and monitor intra-lymph node motility and proliferation of $\mathrm{T}$ cells using intravital two-photon microscopy. This would enhance the resolution of our analysis and allow us to not only compare surface phenotypes between MHC disparate mouse cohorts, but also evaluate kinetics of $\mathrm{T}$ cell mobility and proliferation as they traffic within the lymph node.

An alternative and complementary approach to comparing lymph node and spleen T cell priming capacities between mice with and without $\mathrm{D}^{\mathrm{k}}$-licensed NK cell responses would be to isolate CD11c+ DCs from both organs 2 and $4 \mathrm{~d}$ post-MCMV-OVA infection and co-culture DCs with naïve OT-I T cells. T cell proliferation would be measured via carboxyfluorescein diacetate succinimidyl ester (CFSE) dilution to gauge organ-specific priming efficiencies of DCs. This experimental approach would manipulate natural T cell contact and interactions with DCs, which may mask distinctions in how T cells traffic in respective lymphoid organs. Nonetheless, we expect comparisons of spleen and lymph node DC priming efficiencies may reveal distinct or shared characteristics that inform our understanding of tissue-specific T cell priming.

In light of these proposed areas for future investigation, there is much that remains to be understood with regard to host-pathogen interactions that influence $\mathrm{T}$ cell 
immunity. We must delve deeper into the molecular distinctions that impact DC maturation and function, resolve the impact of antigen abundance and persistence, and examine the impacts of organ-specific priming environments on $\mathrm{T}$ cell immunity. All of these components are undoubtedly interconnected factors of the gestalt profile of CD8+ T cells. As we develop a more complete awareness of these various immune modifiers and how they influence one another, we will advance understanding of the mechanistic underpinnings connecting specific NK cell responses to viral infection with guiding CD8+ T cell immunity.

\section{Early MPEC skewing in mice with efficient licensed NK cell control of MCMV is}

\section{IL-10 dependent}

In addition to the multitude of co-stimulatory signals needed for guiding CD8+ $\mathrm{T}$ cell memory differentiation during primary infection, recent studies have highlighted an important role for the anti-inflammatory cytokine IL-10 in mediating MPEC formation $(269,270,303)$. Classical anti-inflammatory programs such as IL-10 are critical for preventing excessive immunopathology and are typically associated with the resolution phase of viral infection (304). Although early induction of IL-10 can delay or impair efficient control of latent MCMV infection, several groups have noted increased weight loss and immune-related tissue pathology in the absence of total or regulatory $\mathrm{T}$ cellderived IL-10, demonstrating an overall host-protective role for this cytokine $(213,305$, 306). Using LCMV-Armstrong infection, Kaech and colleagues further discovered an indirect effect of IL-10 on CD8+ T cell memory differentiation, whereby IL-10 decreased the maturation of DCs (CD80/86, PDL1, PDL2) and reduced DC production of pro- 
inflammatory cytokines, thus shielding activated CD8 $+\mathrm{T}$ cells from prolonged effector differentiating cues (270).

In the present work, we demonstrated similar requirements for IL-10 signaling in order to mediate MPEC differentiation in $\mathrm{D}^{\mathrm{k}}$ mice (Figure 3.5). In the presence of $\alpha$-IL10R neutralizing antibodies, we observed increased numbers of antigen-specific CD8+ T cells, and a greater proportion of these cells were skewed towards KLRG1+ CD127SLECs (Figure 3.5E-G) as seen in non- $\mathrm{D}^{\mathrm{k}}$ mice. Interestingly, despite this increased SLEC skewing for virus-specific CD8+ T cells in mice with IL-10R blocking antibody, the degree of terminal effector cell differentiation (ratio KLRG $1^{\text {hi }}$ : KLRG $1^{10}$ ) remained consistent with isotype-treated $\mathrm{D}^{\mathrm{k}}$ mice (data not shown), indicating an incomplete conversion to the non- $\mathrm{D}^{\mathrm{k}}$ phenotype. Nonetheless, we observed sustained maturation of DCs in IL-10R blocked mice even out to $\mathrm{d} 8$ post-infection (Figure 3.6A-D) as well as sustained expression of mPDCA-1 on all monocytes (Figure 3.6F). We also identified a higher representation of total monocytes in non- $\mathrm{D}^{\mathrm{k}}$ and IL-10R blocked mice, of which $\sim 60-70 \%$ were GR $1^{10} \mathrm{CD} 11 \mathrm{c}$ - patrolling monocytes (Figure 3.6E-I). In comparison, $\mathrm{D}^{\mathrm{k}}$ mice with IL-10 signaling intact exhibited at least half as many total monocytes, with a predominant skew towards GR $1^{\text {inthi }} \mathrm{CD} 11 \mathrm{c}$ - inflammatory monocytes. Together, these data suggest that licensed NK cell regulation of T cell priming is dependent on the antiinflammatory activities of IL-10, and through limiting the duration of co-stimulatory ligand display on DCs and perhaps inflammatory cytokine signaling, the combined activities of licensed NK cells and IL-10 can shape memory T cell differentiation.

Many questions remain to be addressed. First and foremost, it would be informative to determine whether differential production of IL-10 is evident for $\mathrm{D}^{\mathrm{k}}$ and 
non- $\mathrm{D}^{\mathrm{k}}$ mice. With varied kinetics of early viral control and resolution of acute MCMV in the spleen, distinct concentrations of IL-10 in both strains may at least partially explain the early divergence in $\mathrm{CD} 8+\mathrm{T}$ cell differentiation phenotypes and the increased maintenance of KLRG1+ SLECs in non- $\mathrm{D}^{\mathrm{k}}$ mice. Kinetic profiling of cytokine signatures may be necessary to distinguish significant differences in serum IL-10 concentration, and at the very least would inform our understanding of the dynamics of IL-10 production during MCMV infection. While it is possible that differences in IL-10 will not be detected amongst different strains, functional characterizations of the impacts of IL-10 deficiency or receptor blockade in both strains would further clarify potential context-dependent effects of the anti-inflammatory cytokine. Moreover, despite that total serum cytokine levels can be similar between two hosts, the dominant cellular source of IL-10 and the local versus systemic effects of IL-10 may additionally influence the role of this anti-inflammatory cytokine in guiding CD8+ T cell differentiation.

IL-10 can be produced by a variety of immune cells, including CD4+ T cells, B cells, macrophages, dendritic cells, and even NK cells (304). Although this redundancy certainly reflects the importance of maintaining anti-inflammatory programs, distinct periods of peak activity for these different cell subsets likely highlights additional local or cell-specific effects of IL-10. Thus, defining the prominent cellular sources of IL-10 at various times during acute MCMV infection in mice with efficient licensed NK cell control of infection is expected to inform our understanding of the cellular and molecular underpinnings of enhanced, IL-10-dependent MPEC differentiation. FoxP3 $+\mathrm{CD} 4+\mathrm{T}$ regulatory cells are perhaps the most notorious IL-10 producers in response to a variety of infections, however their peak activity is generally detected during adaptive immune 
phases, usually after d $5(270,307)$. With that said, Allam et al. have demonstrated a critical role for regulatory $\mathrm{T}$ cells in dampening innate inflammation during MCMV infection prior to the onset of CD8+ T cell immunity at $\mathrm{d} 7$ (308). Thus, early regulatory T cell recruitment may lessen an otherwise prolonged exposure to co-stimulatory ligands and inflammatory cytokines.

In addition to $\mathrm{T}$ cells, $\mathrm{NK}$ cells can also make abundant IL-10 in response to systemic infections, including MCMV $(220,307)$. Biron and colleagues demonstrated that MCMV-induced IL-10 from NK cells increased during infection coincident with NK proliferation and decreased IFN $\gamma$ production (220). In a related study, NK-derived IL-10 was shown to be host protective via reduction in both viral titers and immunopathologic CD8+ T cell cytokines (221). Given the specificity of $\mathrm{D}^{\mathrm{k}}$-licensed G2+ NK cells for MCMV and the rapid proliferation in response to viral infection, NK cells are prime candidates as the chief producers of IL-10 during early acute MCMV infection. Still, additional immune cells such as macrophages, monocytes, and DCs can provide robust quantities of IL-10 during infection resolution and tissue repair (304).

To determine the source of IL-10 in the present mouse model of licensed NK cell viral control $\left(\mathrm{D}^{\mathrm{k}}-\mathrm{NK} \mathrm{C}^{\mathrm{c} 571}\right)$ a combination of phenotypic and functional assays would be required. Proof of concept studies examining $I l 10$ transcript over a kinetic time course should be undertaken for the cell subsets indicated above as this is a rapid and robust method for determining gene expression. For the populations of interest (i.e. increased Il10 expression), functional assays such as ex vivo incubation of splenocytes from infected mice in the presence of monensin (Golgi plug) could be further pursued to confirm protein translation of IL-10 at the defined times of interest. Cell-specific 
depletion studies could also be utilized to confirm the dominant source(s) of IL-10 during MCMV infection, however, this approach has several caveats depending on the population of interest. Bulk CD4+ T cell depletions (GK1.5 mAb) or CD25+ regulatory T cell-specific depletions (PC61 mAb) should not drastically affect the early kinetics or phenotypes of CD8 $+\mathrm{T}$ cell priming, as seen previously (Fig. 2.6, Wei \& Brown unpublished data). However, Colonna and colleagues have reported protective suppression by regulatory T cells early after MCMV infection (308). Moreover, depletion of NK cells would preclude examination of enhanced CD8+ T cell phenotypes. In the same vein, depletion of myeloid cells through administration of clodronate liposomes may also affect viral levels, as a recent study has defined important roles for inflammatory monocytes in controlling MCMV infection (309). Thus profiling IL-10 producing cells directly, opposed to functional depletion studies, would be preferable. IL-10 reporter mice are commercially available (Jackson, B6.129S6-Il1 ${ }^{\text {tmlFlv }} \mathrm{J}$; tiger mice (310)), and have been instrumental to finding new IL-10 producing cell subsets in response to infection, such as NK cells $(220,307)$. In order to utilize these reporter strains, we could cross the tiger mice to our B6.NKC(l) and B6.NKC(1)- $\mathrm{D}^{\mathrm{k}}$ mice, though this would require careful selection and breeding for the $I l 10$ promoter-GFP cassette, homozygosity for $\mathrm{NKC}^{\mathrm{c} 571}$, and expression of the $\mathrm{D}^{\mathrm{k}}$ transgene. With these reporter mice crossed to our mice with and without licensed NK cell control of MCMV, we could accurately detect prominent and perhaps previously unappreciated cellular producers of IL-10 over a kinetic time course. Moreover, by altering the dose of infection in mice with and without licensed NK cell control as in Figure 3.4, we could assess whether IL- 
10 production by specific cell subsets is qualitatively different between MHC-disparate strains or whether it is a byproduct of the efficient viral control.

Lastly, as a proof of concept for the role of IL-10 in shaping memory CD8+ T cell differentiation during MCMV infection, we can supplement non- $\mathrm{D}^{\mathrm{k}}$ mice with exogenous mouse recombinant IL-10 (rIL-10) to see if we could prevent skewing of CD8+ effector T cells to terminally differentiated SLECs. Prior studies have demonstrated that IL-10 deficient mice mount more robust adaptive immune responses to MCMV and reduce viral levels more efficiently than IL-10 competent mice, though this often comes at the expense of increased immunopathology $(213,305,306)$. Moreover, the influence of IL10 deficiency on memory cell formation has not been previously examined for MCMV. By treating more susceptible mouse strains with exogenous rIL-10, we may promote MPEC formation early during acute infection. It is likely that by boosting the antiinflammatory response we would prolong the duration of acute MCMV infection in the spleens of non- $\mathrm{D}^{\mathrm{k}}$ mice. While extended acute infection could conceivably drive the host requirement for continued SLEC differentiation - similar to how inefficient viral control by NK cells promotes a greater magnitude of CD8+ T cells (106) - early rIL-10 intervention could help to establish a pool of MPECs during a critical period of T cell differentiation, such that this memory pool may be maintained regardless of sustained effector cell proliferation. In support, we did observe a greater pool of memory cells in MCMV-infected $\mathrm{D}^{\mathrm{k}}$ mice compared to non- $\mathrm{D}^{\mathrm{k}}$ mice, despite the fact that both types maintain similar total numbers of KLRG1+ effector cells during latency (Fig. 3.8). This corresponded with an early accumulation of MPECs during MCMV infection (Fig. 3.1). Furthermore, Harty and colleagues have demonstrated that by default, antigen-primed 
$\mathrm{CD} 8+\mathrm{T}$ cells differentiate into long-lived memory $\mathrm{T}$ cells, and that inflammatory cytokines guide the proportion of cells that differentiate into SLECs (297). Moreover, the timing and duration of these inflammatory cytokine cues and consequential costimulatory interactions with DCs influence the total number of memory CD8+ T cells that are formed in response to foreign antigen (311). Thus, the timing of exposure to IL10 during MCMV infection could have long-lasting impacts on CD8+ T cell immunity.

Clarifying the role of IL-10 as it relates to CD8+ T cell differentiation during CMV infection will be important as we seek to understand the molecular basis for how licensed NK cells influence T cell immunity. This could provide an important target for therapeutic intervention as we seek to alter protective $\mathrm{CD} 8+\mathrm{T}$ cell immunity, particularly if licensed responses to infection alter IL-10 kinetics. It should be noted that human (H)CMV encodes an IL-10 homologue that can dampen immune responses to infection, and is thought to promote viral persistence $(312,313)$. HCMV-derived IL-10 has been shown to upregulate human monocyte production of IL-10 as well, which can further suppress immune control of CMV (314). Whether or not HCMV-derived IL-10 impacts on $\mathrm{T}$ cell differentiation during early acute infection remains to be determined, and will need to be taken into consideration as we consider interventional treatments that modify anti-inflammatory immune signals.

\section{Quality versus quantity: The imprint of licensed NK cell-mediated resistance on CD8+ T cell memory}

In this work, we have demonstrated that specific licensed NK cell responses to MCMV infection support early accumulation and memory differentiation of antiviral 
CD8+ T cells (Chapter III). Moreover, we found that these early impacts on T cells were durable throughout viral latency, such that the majority of virus-specific CD8+ T cells were $\mathrm{CD} 127+$ memory cells. In contrast, mice that were either lacking $\mathrm{D}^{\mathrm{k}}$ or were specifically depleted of G2+ NK cells during acute infection maintained less than half the number of virus-specific CD8+ T cells during viral latency, and a majority of cells retained a KLRG1+ effector phenotype. Despite these distinctions in antiviral cell numbers and phenotype, however, transfer of bulk CD8+ T cells from either experimental cohort was protective against secondary viral challenge, though to varying degrees depending on the conditions of viral infection (Fig. 3.10). To our surprise, T cells primed in the absence of licensed NK cells were quicker to control secondary viral infection when challenged immediately after adoptive transfer. We attributed this observation to the higher proportion of KLRG1+ effector cells, and reasoned that with viral infection immediately following the transfer, these effector cells remained in a poised state to respond. In contrast, $\mathrm{T}$ cells primed in donors with $\mathrm{D}^{\mathrm{k}}$-licensed $\mathrm{NK}$ cells intact were more effective at controlling secondary viral challenge, but only when viral infection was delayed after transfer. Under these circumstances, we inferred that in the absence of previous viral infection and prior to secondary challenge, transferred effector cells contracted due to a lack of antigen stimulation and only CD127+ virus-specific CD8+ T cells remained. Thus, given the higher proportion of memory cells from $\mathrm{D}^{\mathrm{k}}$ donors with G2+ NK cells intact, these donor cells now conferred improved viral control.

Thus far we have investigated the protective capacity of bulk transferred CD8+ T cells from donors with disparate NK cell responses to acute MCMV infection. While these transferred populations comprised skewed proportions of effector and memory $\mathrm{T}$ 
cells, in regard to individual host-immunity, similar numbers of total effector T cells were actually maintained in both experimental groups. The proportions were offset by the increased presence of virus-specific memory cells in mice with $\mathrm{D}^{\mathrm{k}}$-licensed G2+ NK cells (Fig. 3.8). In a previous study investigating the protective efficacy of CD $27+$ memory $T$ cells versus KLRG1+ effector cells, it was demonstrated that single transfers of either population into naïve $\mathrm{Rag}^{-/-}$mice was sufficient to protect against a lethal dose of MCMV (315). Moreover, studies addressing the protective capacities of primary and secondary memory CD $8+\mathrm{T}$ cells have revealed that secondary memory and short-boosted memory cells retain poised cytolytic capabilities, and are better equipped to protect against acute viral infection $(159,163,316)$. The tradeoff for these secondary memory cells, however, is reduced longevity of memory maintenance (163) and increased susceptibility to exhaustion during chronic infection (316). Therefore, it is possible that while both mouse strains could mount similar immediate effector responses to secondary infection, only mice with $\mathrm{T}$ cells primed in the presence of licensed NK cells may retain long-term memory cells with improved stem-like qualities.

To investigate this possibility, we could repeat transfers of congenically marked (CD45.2) bulk CD8+ T cells from previously infected, MHC-disparate donor mice into MHC-matched recipients (CD45.1), and immediately re-challenge with high dose MCMV, as described in Figure 3.10A. However, rather than suspending the experiment at $\mathrm{d} 5$, we could follow the secondary infection to viral latency (d 56) and assess differentiation profiles of the donor-derived (CD45.2) T cells. Importantly, we would want to examine the frequency of donor-derived secondary memory $\mathrm{T}$ cells present during viral latency in the recipient strains. We could also extend our $\mathrm{T}$ cell profiling to 
include intracellular analysis of fate skewing transcription factors, such as Tbet, Eomes, and Blimp-1, as described previously (317). Furthermore, we could expand the investigation to inspect tertiary memory via repeated transfer of the bulk donor-derived $\mathrm{T}$ cells into matched recipients and challenge with a third dose of MCMV. If the higher proportion of memory cells from $\mathrm{D}^{\mathrm{k}}$ mice demonstrates improved maintenance and replenishment of the memory compartment, we would expect to see a consistently higher frequency and number of memory cells after successive viral challenges. To increase our cell recovery and transfer efficiency, we could seed congenically marked OT-I T cells (CD45.1) into $\mathrm{D}^{\mathrm{k}}$ mice (CD45.2) with and without $\mathrm{G} 2+\mathrm{NK}$ cell depletion prior to infection with MCMV-OVA; secondary and tertiary bulk OT-I transfers could then be performed as described above and consistent with previous studies (315). Utilizing the OT-I and MCMV-OVA systems would also control for potential differences in immunodominant peptides resulting from initial NK cell responses to infection, which could also influence $\mathrm{T}$ cell immunity beyond the general effector and memory differentiation spectrum.

In addition to the quantitative differences in memory $\mathrm{T}$ cell formation that the current work highlights, we are also very curious about potential qualitative differences precipitated by disparate NK responses to acute MCMV. Typically, the first property assessed for $\mathrm{CD} 8+\mathrm{T}$ cell immunity is 'magnitude' (i.e. how many cells are available to mediate the effect: total CD8+ T cells, virus-specific CD8+ T cells, IFN $\gamma+/ T N F \alpha+C D 8+$ T cells, effector vs. memory, etc). However, the quality within a defined population can vary greatly depending on the phenotypic or functional parameters investigated, and as our technological capabilities to better resolve those sub-populations increases - namely 
through flow cytometry and single cell sequencing - we will undoubtedly develop more refined profiles to assess the quality of $\mathrm{CD} 8+\mathrm{T}$ cell responses (318).

To further explore potential qualitative differences between CD8+ T cells primed in mice with and without licensed NK cell MCMV control, we could equalize the numbers of defined CD8+ T cell subsets prior to transfer to exclude the effect of quantitative differences between donors. That is, rather than transferring equal numbers of bulk CD8 $+\mathrm{T}$ cells, we could specifically sort CD127+ antigen experienced CD11c+ $\mathrm{CD} 44+$ cells from infected mice and transfer them in equal numbers into MHC-matched recipient mice. At a rudimentary level, this would allow us to assess whether the quality of CD8+ memory T cell priming - versus effector cells - is similar between strains. Similar levels of body weight recovery and viral control might suggest that the impact of licensed NK cells on $\mathrm{T}$ cell differentiation is predominantly quantitative rather than qualitative. On the other hand, sustained differences in viral control could suggest that memory T cells primed in different host settings at the level of the NK cell are qualitatively unique. One caveat to this approach stems from the limiting definition of the defined population, such that CD127 may be just as broad as CD8, and additional markers or indications may need to be screened to narrow down the precise population of interest. Nonetheless, this approach is likely to inform our understanding of whether or not broadly defined memory cell populations are qualitatively distinct. We expect these studies to open new avenues for research and discovery. As discussed above, an OTI/MCMV-OVA system would also help to rule out potential differences in distinct clonal repertoires primed in the disparate settings. 
If differences in memory $\mathrm{CD} 8+\mathrm{T}$ cell quality are observed even when the quantity transferred of cells is equal, it would be of significant interest and importance to further characterize $\mathrm{T}$ cell proliferation kinetics, differentiation, and function after rechallenge. We could label cells with CFSE prior to transfer in order to examine the kinetics and magnitude of memory cell proliferation. Similarly, we could interrogate functional cytolytic capacities of the transferred memory cells through in vivo cytotoxicity assays or ex vivo ${ }^{51} \mathrm{Cr}$-release assays using peptide-pulsed target cells at various times post-infection. These assays, in addition to the phenotypic profiling described in Chapters II and III, would help develop our understanding of intrinsic differences associated with memory CD8 $+\mathrm{T}$ cells primed in the presence or absence of licensed NK cells control of viral infection, and the quality of effector cells derived from them.

Additionally, it would be of special interest to investigate localization of transferred memory CD8 $+\mathrm{T}$ cells prior to and during secondary MCMV challenge, and to determine if distinct priming of memory cells results in unique trafficking patterns. In the present work, we modified our $\mathrm{T}$ cell challenge assays to diminish the presence of KLRG1+ effector cells by delaying infection by one week after transfer (Fig. 3.10C). Remarkably, while we demonstrated improved protection by memory cells primed in the presence of licensed G2+ NK cells, we also observed that the kinetics of memory cell control were more efficient than seen previously with viral challenge immediately after cell transfer (Fig. 3.10B,D). This may indicate that improved trafficking and localization of memory cells to defined lymphoid regions is necessary to mediate efficient antiviral protection. von Andrian and colleagues have previously demonstrated that CXCR3+ 
central memory $\mathrm{T}$ cells preferentially localize to the periphery of lymph nodes via CXCL9 and CXCL10 gradients, where they can encounter and respond to viral antigens more efficiently than CXCR3- naïve T cells (319). Examining histological sections of the spleen and lymph nodes to identify the location of transferred $\mathrm{T}$ cell populations through immunofluorescence for the congenic marker of choice (e.g. CD45.1) we could readily compare local deposits of $\mathrm{T}$ cells primed in mice with disparate $\mathrm{NK}$ responses to MCMV. These histological studies could be further advanced in combination with MCMV-GFP infections to identify if memory $\mathrm{T}$ cell populations are co-localizing with specific sites of infection, as previously described (103).

Similar to the qualitative investigations of memory $\mathrm{T}$ cells, it would be interesting to explore the potential differences in poised cytolytic capabilities of MCMV-specific effector cells. Although mice with and without efficient NK-mediated viral control exhibited similar numbers of total antiviral CD8+ effector T cells, differences in acute viral control may have impacted the systemic abundance of viral antigen. Previous studies have established that non-replicative and spread-deficient MCMV infection can drive memory $\mathrm{T}$ cell inflation and terminal SLEC differentiation through repeated antigen stimulation $(207,208)$. Thus, a greater antigenic burden established early during acute infection could potentially create a repetitive boosting environment, which may impact effector cell poise for lytic activities. Certainly we could repeat the abovementioned experiments transferring effector $\mathrm{T}$ cells instead of memory $\mathrm{T}$ cells, and we could directly assess the efficiency of host protection. Alternatively, to directly test cytolytic performance, we could utilize in vivo cytotoxicity assays with CFSE labeled peptidepulsed target cells. If no differences in killing efficiency are observed, we can likely 
attribute prior anti-viral distinctions to quantitative differences. However, if we detect substantial discrepancies in the cytolytic capacity or kinetics of differently primed effector cells, further study is warranted to test the effects of antigen burden on effector cell performance. Such experiments may include modifying MCMV doses to manipulate antigen set points or transferring MCMV-OVA primed OT-I cells into matched infection recipients with or without $\mathrm{G} 2+\mathrm{NK}$ cells depletion to assess the impact of differential antigen load on the long-term stimulation of effector cells.

\section{Longevity of licensed NK cell influence on $\mathbf{T}$ cell differentiation and maintenance of memory cells}

We have presented evidence in this work that licensed NK cell responses to acute viral infection yield significant influence over the kinetics of T cell priming, accumulation, and differentiation through the manipulation of inflammatory and costimulatory pathways (Chapters II and III). This has been demonstrated in mice with or

without the critical $\mathrm{D}^{\mathrm{k}}$-licensing ligand expressed. Moreover, specific depletion of $\mathrm{G} 2+$ NK cells solely during acute infection recapitulated the dominant phenotypes described herein (Fig. 3.3, Fig. 3.9). Importantly, with only two doses of G2+ NK cell-depleting antibody during infection ( $\mathrm{d}-2 \& \mathrm{~d}$; 4 DD11 mAb) we observe a recovery of G2+ NK cells between 6-10 d post-infection (data not shown). While the focus of this work has examined the impact of NK cells on CD8+ T cell immunity during the first $6 \mathrm{~d}$ of acute infection, it remains to be determined whether or not licensed G2+ NK cells have an enduring role in shaping the virus-specific CD8 $+\mathrm{T}$ cells beyond acute infection. 
Other groups have examined the suppressive influence of NK cells on CD8+ T cell immunity during certain viral infections (e.g. LCMV, Pichinde virus) and have reported early impacts of NK cells on T cell immunity, demonstrating that NK depletion after 2-3 $\mathrm{d}$ of infection is insufficient to reverse their effects $(13,14,18)$. Although the emphasis of those studies was on NK suppression of APCs, CD4+ T, and CD8+ T cells, their studies nonetheless identified a restricted window for when NK cells exert their greatest effect on adaptive immunity. Waggoner et al. demonstrated a 'rheostat' regulation role for NK cells, such that the dose and magnitude of viral infection influenced whether NK impairment of $\mathrm{CD} 8+\mathrm{T}$ cells was detrimental (moderate dose of infection) or immune-protective to the host through limitation of CD8+ T-mediated immunopathology (high dose) (13). However, these studies were typically terminated between 7-14 d post-infection, and did not examine a long-term role for NK cells in shaping $\mathrm{T}$ cell activities. The duration of $\mathrm{NK}$ impacts on $\mathrm{T}$ cells is particularly pertinent for persistent and chronic viral infections, such as HIV. Evidence of prolonged NK cell activation during chronic HIV infection has been reported (320), though dysfunctional NK performance in highly viremic patients has been shown to negatively affect APC stimulatory capacity and subsequent CD8+ T cell immunity $(261,321)$. Although MCMV establishes latency and is not considered a chronic viral infection, NK cells do exhibit memory-like features after MCMV infection(11,279), and they may continue to shape $\mathrm{T}$ cell immunity

To investigate a potential role for $\mathrm{G} 2+\mathrm{NK}$ cells in shaping $\mathrm{T}$ cell responses beyond acute infection, we could repeat our MCMV infections in mice with $\mathrm{D}^{\mathrm{k}}$ expressed and treat them with isotype or $\alpha-\mathrm{G} 2 \mathrm{mAb}$ for a prolonged period of time. This approach 
would allow us to compare CD8 $+\mathrm{T}$ cell phenotypes without depletion, with a single depleting dose, and with sustained depletion of G2+ NK cells. In the event that serum sickness complicates the analysis, we could also transfer primed virus-specific CD8+ T cells from the described experimental groups into infection matched Ly49G2-deficient mice with $\mathrm{D}^{\mathrm{k}}$ expressed (Cronk \& Brown, unpublished data). In either case, we would be particularly interested to investigate the effector cell phenotypes in mice with sustained G2+ NK depletion. Previously, we discussed the possibility that virus-specific CD8+ T cells were maintained at higher frequencies in $\mathrm{D}^{\mathrm{k}}$ mice due to MHC-I inhibition of G2+ NK cells (Chapter III). In the absence of this inhibition, however, and perhaps more importantly in the absence of type I IFN-mediated upregulation of MHC-I beyond acute infection, it is possible that these effector cells would be targeted for NK-mediated lysis. Using these strategies we could determine whether G2+ NK cells have an ongoing role in molding $\mathrm{T}$ cell immunity, or whether their impact on $\mathrm{T}$ cell immunity is truly determined within the first 3 days of an infection.

\section{Closing remarks}

In conclusion, the data and perspectives presented herein provide significant insights and advances to our understanding of self-licensed NK cell responses to viral infection, and how the quality of those responses informs adaptive immune cell priming, differentiation, and long-term protective immunity. We have demonstrated that even with efficient restriction of acute MCMV infection, licensed NK cells can engender a robust and lasting virus-specific $\mathrm{T}$ cell response to MCMV that is protective upon secondary challenge. Moreover, we discovered that licensed NK cells exert considerable influence 
on the environment in which CD8+ T cells are primed, such that they can influence the duration of co-stimulatory ligand expression and even the requirements for specific costimulatory signals to prime $\mathrm{T}$ cells during acute viral infection. Despite demonstrating similar efficiencies of viral resistance these investigations are distinct from previous studies in both the model of specific licensed NK cell-mediated viral control - opposed to dominant activating receptor recognition of infected targets - as well as the general restrictive effect of efficient viral control of MCMV on T cell immunity $(23,106,109$, 224). Thus, viral control in and of itself is not predictive of providing benefit to $\mathrm{T}$ cell immunity. Rather, we believe that the manner in which licensed NK cells respond to MCMV infection offers unique benefits to $\mathrm{T}$ cell priming and differentiation, such that they permit enough viral antigen and inflammation to prime $\mathrm{T}$ cell immunity without exhausting or terminally differentiating these cells as short-lived effector cells.

Interestingly, preliminary studies have suggested two parallel impacts of licensed NK cell-mediated viral control: (i) Efficient viral control preserves or supports recovery of DCs such that the abundance of DCs impacts virus-specific T cell kinetics and magnitude. Whether this phenomenon is directly related to viable DC numbers, or whether this is a manifestation of the preservation of secondary lymphoid organ architecture remains to be determined; it is likely that both factors are involved. (ii) Licensed NK cell responses to MCMV direct the differentiation of effector cells into SLECs or MPECs. This latter impact is less well understood, as sustained elevation of viral levels in mice challenged with high dose MCMV would be expected to amplify inflammatory signals and cytokines that drive terminal differentiation of $\mathrm{T}$ cells as SLECs. An intriguing hypothesis is that licensed NK cells can prune and select for 
specific profiles of DCs or T cells (256). NK cells dominantly guided by activating receptors may readily kill target cells or activated lymphocytes due to the lower expression of self-specific inhibitory receptors $(82,83)$. In contrast, licensed NK cells are inherently marked by their expression of self-specific inhibitory receptors, which may allow them to discriminate more selectively amongst mature DCs and activated T cells that are like to promote beneficial, but not immunopathogenic, immune responses. Further studies are warranted to explore this latter possibility.

Finally, as we look to improve vaccination strategies and develop novel immunotherapeutic treatments in humans to elevate protective CD8 $+\mathrm{T}$ cell immunity, it has become increasingly evident that NK cells can determine the fate of T cells. Furthermore, the compounded diversity of human NK responses guided by HLA and NKC polymorphism adds to spectrum of NK cell responses observed in various virus infections and tumor settings. While experimental knockout and depletion studies in inbred mouse strains are critical to study of the impact that these innate lymphocytes can have on $\mathrm{T}$ cell immunity, it should be understood that human NK responses are extremely diverse and exquisitely tuned to unique host environments. Thus, interventional therapies aimed to modify NK function as a means to boost $\mathrm{T}$ cell immunity, while helpful in certain contexts, may be damaging in others, and the factors guiding NK responses should be thoroughly considered before ever manipulating this potent innate effector cell. 


\section{Chapter V}

Materials and Methods 
Ethics Statement. All mouse experiments conducted in this investigation were carried out in accordance with the Animal Welfare Act and the recommendations in the Guide for Care and Use of Laboratory Animals of the National Institutes of Health.

Experimental procedures and in vivo treatments were approved by the University of Virginia Animal Care and Use Committee (Protocol Number: \#3050).

Mice. All mice used in this study were bred and maintained under specific pathogen-free conditions at the University of Virginia. C57L-derived MHC I D ${ }^{\mathrm{k}}$ congenic $(\mathrm{R} 7)$ and $\mathrm{D}^{\mathrm{k}}$ transgenic (L.Tg1 and L.Tg3) mouse strains were described previously $(71,78)$. C57B1/6 (B6). $N k c^{c 57 l}\left(\mathrm{NKC}^{l}\right)$ and B6.Cg-Nkc $c^{c 57 l}-D^{k}\left(\mathrm{NKC}-\mathrm{D}^{\mathrm{k}}\right)$ congenic mice with wild-type CD27 were generated by introgressing a C57L natural killer gene complex (NKC) into the B6 background using genome-wide marker-assisted selection as described previously $(78,322)$. Where indicated, these mice were compared to $\mathrm{D}^{\mathrm{k}}$-disparate B6.Cd27\%$N k c^{129 / P 2 O l a}(\mathrm{CD} 27 \mathrm{KO})$ and B6.Cg-Cd27-/ $-N k c^{129 / \text { P2Ola }_{-}}-D^{k}\left(\mathrm{CD} 27 \mathrm{KO}-\mathrm{D}^{\mathrm{k}}\right)$ mice. CD27 KO mice, which had been previously backcrossed to B6 from 129/P2Ola-founders, retain a CD27-linked $\mathrm{NKC}^{129}$ on chromosome $6(235,239)$ and were kindly provided by Jannie Borst (The Netherlands Cancer Institute, Amsterdam, Netherlands) via Ross Kedl (University of Colorado-Denver, CO, USA) (254). Importantly, $N K C$-Ly49 haplotypes in 129 and C57L are highly related (323), Ly49g alleles in 129 and C57L mice are identical $(78,324)$, and both $\mathrm{G} 2$ receptors specifically bind $\mathrm{D}^{\mathrm{k}}(80)$. CD27 KO mice were thus crossed to $\mathrm{B} 6 . \mathrm{D}^{\mathrm{k}}$ mice (a by-product of $\mathrm{NKC}^{l}-\mathrm{D}^{\mathrm{k}}$ production) to generate $\mathrm{B} 6 . \mathrm{Cg}-\mathrm{Cd} 2 \mathrm{~T}^{-/}$$N k c^{129 / P_{2 O l a}}-D^{k}\left(\mathrm{CD} 27 \mathrm{KO}-\mathrm{D}^{\mathrm{k}}\right)$ mice. Of note, both 129- and C57L-derived NKC haplotypes lack a Ly49h gene and, consequently, Ly49H+ NK-mediated MCMV 
resistance. All mice in this study were managed using a Colony Management System (Jackson Labs, JCMS Access, Version 6.1.9). All protocols were approved by the IACUC.

Virus infection and antibody treatments. Smith strain MCMV salivary gland stock virus (SGV) was prepared and titered on NIH-3T3 cell monolayers as described (77). SGV was administered via i.p. injection of $2 \times 10^{4}$ PFU. Where indicated, neutralizing mAbs specific for CD70 (mAb FR70; $250 \mu \mathrm{g}$ /dose i.p. injected on 0, 2 and $4 \mathrm{~d}$ after infection), CD80 (mAb 16-10A1, BioXCell; $200 \mu \mathrm{g} /$ dose i.p. injected on 0 and $3 \mathrm{~d}$ after infection), CD86 (mAb GL1, BioXCell; $200 \mu \mathrm{g} /$ dose i.p. injected on 0 and $3 \mathrm{~d}$ after infection), CD40L (mAb MR1, BioXCell; $250 \mu \mathrm{g} /$ dose on 0,2 and $4 \mathrm{~d}$ after infection), and IL-10 receptor (mAb 1B1.3A, BioXCell; $250 \mu \mathrm{g}$ were administered on 0, 2, 4, and 6 $\mathrm{d}$ after infection). For in vivo $\mathrm{G} 2+\mathrm{NK}$ cell depletions, $200 \mu \mathrm{g}$ mAb AT8 or mAb 4D11 were i.p. injected $2 \mathrm{~d}$ prior and on the day of infection. For in vivo CD4+ T-cell depletions, $200 \mu \mathrm{g}$ of mAb GK1.5 (UVA Antibody Engineering and Technology Core) were i.p. injected on $\mathrm{d} 5,4$, and 0 before infection. Control IgG from rat serum (Sigma Life Sciences) or Syrian Hamster serum (Jackson ImmunoResearch Laboratories, Inc.) was administered in equivalent dose regimens, accordingly. Lymphocyte depletions exceeded 95-99\% efficiency.

Flow cytometry and antibodies. Spleens were harvested from mice at the indicated time points postinfection and homogenized into single cell suspension through nylon cell strainers (Falcon Corning Brand; Life Sciences). Analyses of dendritic cells required 
additional processing with Collagenase $\mathrm{D}(0.5 \mathrm{mg} / \mathrm{mL}$; Roche $)$, as previously described (241). Single cell suspensions were pre-blocked with Fc receptor blocking antibody (2G9; UVA Lymphocyte Culture Center, Charlottesville, VA). All antibody incubations were performed on ice, and cells were washed with PBS or sorting buffer after each stain. Labeled cells were analyzed using the BD FACS Canto II (BD BioSciences) and the CytoFLEX (Beckman Coulter, Inc.). Data were collected using FACSDiva software (v8.0; BD BioSciences) or CytExpert software (v1.2.8.0; Beckman Coulter, Inc.) and analyzed with FlowJo (Versions 9.7.2 and 10.1; FlowJo LLC).

Fluorescently labeled and biotin-conjugated antibodies were purchased from BioLegend (San Diego, CA), BD Biosciences (San Diego, CA), and eBiosciences (San Diego, CA). Antibodies included anti-CD3 (145-2C11), CD19 (6D5), CD8 (53-6.7), CD4 (GK1.5; RM4-4), NKp46 (29A1.4), CD11b (M1/70), CD27 (LG.7F9), Ly49G2 (4D11), CD44 (IM7), CD11c (N418), KLRG1 (2F1), CD127 (A7R34), IFN $\gamma$ (XMG1.2), TNF $\alpha$ (MP6-XT22), CD40L (MR1), CD49b (DX5), CD69 (H1.2F3), CD70 (FR70), CD86 (GL-1), MHC II I-A/I-E (2G9), CD317 (129c1), GR-1: Ly6C/G (RB6-8C5), and

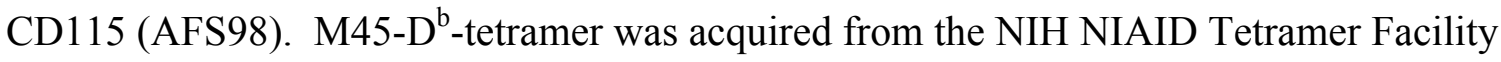
(Bethesda, MD). LIVE/DEAD Fixable Violet and Aqua Dead Cell staining kits were purchased from ThermoFisher Scientific (Waltham, MA).

Peptide restimulation assays. Single cell suspensions of mouse splenocytes from d 6 infected mice were incubated with either immunodominant M45 peptide (HGIRNASFI) or less immunodominant m139 (TVYGFCLL), M38 (SSPPMFRV), and IE3 (RALEYKNL) peptides as described previously (24). Briefly, splenocytes were 
resuspended in modified IMDM (ThermoFisher Scientific), Brefeldin A (5 $\mu \mathrm{g} / \mathrm{mL}$; BD Biosciences), and the indicated MCMV-derived peptide and subsequently incubated at $37^{\circ} \mathrm{C}, 5 \% \mathrm{CO}_{2}$ for 5 hours. Cytokine production was analyzed via intracellular cytokine staining. Surface proteins and fixable live/dead stains were performed first, as described above. Cells were then fixed and permeabilized (BD CytoFix/Cytoperm Kit) and subsequently stained on ice for IFN $\gamma$ and TNF $\alpha$.

Adoptive cell transfers. CD8 $+\mathrm{T}$ cells were isolated from the pooled spleens of 3-5 mice within a cohort. Splenocytes were prepared into single cell suspension as described above. CD8+ T cells were negatively enriched on MACS LS columns (Miltenyi Biotec, CD8 $+\mathrm{T}$ Cell Isolation Kit). Cell purity was confirmed $>95 \%$ by flow cytometric analysis for all cohorts. $5 \times 10^{6} \mathrm{CD} 8+\mathrm{T}$ cells from each cohort were transferred intravenously (i.v.) to naïve MHC-I matched recipients. Recipients were then rested as indicated, prior to i.p. MCMV challenge.

Nucleic acid isolation and Quantitative PCR. Spleen DNA was prepared and analyzed for MCMV genomes using quantitative (q)PCR as previously described (325). For analysis of $C d 70$ expression, splenic $\mathrm{CD} 11 \mathrm{c}+\mathrm{DCs}$ were positively selected using CD11c+ MACS microbeads (Miltenyi Biotec, San Diego, CA). RNA was isolated using TRIzol (ThermoFisher Scientific) according to manufacturer guidelines, and converted to cDNA using Advantage RT for PCR Kit (Clontech, Mountain View, CA). Cd70 cDNA was amplified using gene-specific primers: $C d 70$-Forward, 5'-TGC TGT TGG TTT CAT TGT AGC G-3'; Cd70-Reverse, 5'-ATC CTG GAG TTG TGG TCA AGG G-3', as 
reported (326). Hprt was also amplified using gene-specific primers (71) and used to normalize and compare $C d 70$ expression in infected and naïve DCs.

Statistical methods. All statistical measures were produced in Prism (GraphPad software, v6.0d). Graphs depict mean ( $\pm \mathrm{SD})$ for all data shown. Experimental phenotypes were tested for statistical significance using one-way ANOVA in conjunction with Tukey's post-hoc test, unless otherwise noted. Unpaired student t-tests were used to assess differences between viral titers where appropriate. 


\section{References}

1. Vivier, E., D. H. Raulet, A. Moretta, M. A. Caligiuri, L. Zitvogel, L. L. Lanier, W. M. Yokoyama, and S. Ugolini. 2011. Innate or adaptive immunity? The example of natural killer cells. Science 331: 44-49.

2. Tscharke, D. C., N. P. Croft, P. C. Doherty, and N. L. La Gruta. 2015. Sizing up the key determinants of the CD8(+) T cell response. Nat Rev Immunol 15: 705-716.

3. Welsh, R. M., J. O. Brubaker, M. Vargas-Cortes, and C. L. O'Donnell. 1991. Natural killer (NK) cell response to virus infections in mice with severe combined immunodeficiency. The stimulation of NK cells and the NK cell-dependent control of virus infections occur independently of T and B cell function. $J$ Exp Med 173: 10531063.

4. Bukowski, J. F., B. A. Woda, S. Habu, K. Okumura, and R. M. Welsh. 1983. Natural killer cell depletion enhances virus synthesis and virus-induced hepatitis in vivo. $J$ Immunol 131: 1531-1538.

5. Reddehase, M. J., W. Mutter, K. Münch, H. J. Bühring, and U. H. Koszinowski. 1987. CD8-positive T lymphocytes specific for murine cytomegalovirus immediate-early antigens mediate protective immunity. J Virol 61: 3102-3108.

6. Orange, J. S., M. S. Fassett, L. A. Koopman, J. E. Boyson, and J. L. Strominger. 2002. Viral evasion of natural killer cells. Nat Immunol 3: 1006-1012.

7. Horst, D., M. C. Verweij, A. J. Davison, M. E. Ressing, and E. J. Wiertz. 2011. Viral evasion of T cell immunity: ancient mechanisms offering new applications. Curr Opin Immunol 23: 96-103.

8. Iwasaki, A., and R. Medzhitov. 2015. Control of adaptive immunity by the innate immune system. Nat Immunol 16: 343-353.

9. Medzhitov, R., and C. A. Janeway. 1997. Innate immunity: the virtues of a nonclonal system of recognition. Cell 91: 295-298.

10. Bekiaris, V., O. Timoshenko, T. Z. Hou, K. Toellner, S. Shakib, F. Gaspal, F. M. McConnell, S. M. Parnell, D. Withers, C. D. Buckley, C. Sweet, W. M. Yokoyama, G. Anderson, and P. J. Lane. 2008. Ly49H+ NK cells migrate to and protect splenic white pulp stroma from murine cytomegalovirus infection. J Immunol 180: 6768-6776.

11. Cook, K. D., S. N. Waggoner, and J. K. Whitmire. 2014. NK cells and their ability to modulate $\mathrm{T}$ cells during virus infections. .

12. Crouse, J., H. C. Xu, P. A. Lang, and A. Oxenius. 2015. NK cells regulating T cell responses: mechanisms and outcome. .

13. Waggoner, S. N., M. Cornberg, L. K. Selin, and R. M. Welsh. 2012. Natural killer cells act as rheostats modulating antiviral $\mathrm{T}$ cells. .

14. Rydyznski, C., K. A. Daniels, E. P. Karmele, T. R. Brooks, S. E. Mahl, M. T. Moran, C. Li, R. Sutiwisesak, R. M. Welsh, and S. N. Waggoner. 2015. Generation of cellular immune memory and B-cell immunity is impaired by natural killer cells. . 
15. Lang, P. A., K. S. Lang, H. C. Xu, M. Grusdat, I. A. Parish, M. Recher, A. R. Elford, S. Dhanji, N. Shaabani, C. W. Tran, D. Dissanayake, R. Rahbar, M. Ghazarian, A. Brüstle, J. Fine, P. Chen, C. T. Weaver, C. Klose, A. Diefenbach, D. Häussinger, J. R. Carlyle, S. M. Kaech, T. W. Mak, and P. S. Ohashi. 2012. Natural killer cell activation enhances immune pathology and promotes chronic infection by limiting CD8+ T-cell immunity. .

16. Xu, H. C., M. Grusdat, A. A. Pandyra, R. Polz, J. Huang, P. Sharma, R. Deenen, K. Köhrer, R. Rahbar, A. Diefenbach, K. Gibbert, M. Löhning, L. Höcker, Z. Waibler, D. Häussinger, T. W. Mak, P. S. Ohashi, K. S. Lang, and P. A. Lang. 2014. Type I interferon protects antiviral CD8+ T cells from NK cell cytotoxicity. .

17. Crouse, J., G. Bedenikovic, M. Wiesel, M. Ibberson, I. Xenarios, D. Von Laer, U. Kalinke, E. Vivier, S. Jonjic, and A. Oxenius. 2014. Type I interferons protect T cells against NK cell attack mediated by the activating receptor NCR1. .

18. Cook, K. D., and J. K. Whitmire. 2013. The depletion of NK cells prevents T cell exhaustion to efficiently control disseminating virus infection. .

19. Soderquest, K., T. Walzer, B. Zafirova, L. S. Klavinskis, B. Polić, E. Vivier, G. M. Lord, and A. Martín-Fontecha. 2011. Cutting edge: CD8+ T cell priming in the absence of NK cells leads to enhanced memory responses. .

20. Mocikat, R., H. Braumüller, A. Gumy, O. Egeter, H. Ziegler, U. Reusch, A. Bubeck, J. Louis, R. Mailhammer, G. Riethmüller, U. Koszinowski, and M. Röcken. 2003.

Natural killer cells activated by MHC class I(low) targets prime dendritic cells to induce protective CD8 T cell responses. Immunity 19: 561-569.

21. Adam, C., S. King, T. Allgeier, H. Braumüller, C. Lüking, J. Mysliwietz, A.

Kriegeskorte, D. H. Busch, M. Röcken, and R. Mocikat. 2005. DC-NK cell cross talk as a novel CD4+ T-cell-independent pathway for antitumor CTL induction. Blood 106: 338344.

22. Morandi, B., L. Mortara, P. Carrega, C. Cantoni, G. Costa, R. S. Accolla, M. C. Mingari, S. Ferrini, L. Moretta, and G. Ferlazzo. 2009. NK cells provide helper signal for CD8+ T cells by inducing the expression of membrane-bound IL-15 on DCs. Int Immunol 21: 599-606.

23. Robbins, S. H., G. Bessou, A. Cornillon, N. Zucchini, B. Rupp, Z. Ruzsics, T. Sacher, E. Tomasello, E. Vivier, U. H. Koszinowski, and M. Dalod. 2007. Natural killer cells promote early CD8 T cell responses against cytomegalovirus. PLoS Pathog 3: e123.

24. Stadnisky, M. D., X. Xie, E. R. Coats, T. N. Bullock, and M. G. Brown. 2011. Self MHC class I-licensed NK cells enhance adaptive CD8 T-cell viral immunity. Blood 117: 5133-5141.

25. Trinchieri, G. 1989. Biology of natural killer cells. Adv Immunol 47: 187-376.

26. Lanier, L. L. 2008. Up on the tightrope: natural killer cell activation and inhibition. Nat Immunol 9: 495-502.

27. Lanier, L. L. 2005. NK cell recognition. Annu Rev Immunol 23: 225-274.

28. Parham, P. 2008. The genetic and evolutionary balances in human NK cell receptor 
diversity. Semin Immunol 20: 311-316.

29. Bryceson, Y. T., and E. O. Long. 2008. Line of attack: NK cell specificity and integration of signals. Curr Opin Immunol 20: 344-352.

30. Herberman, R. B., M. E. Nunn, and D. H. Lavrin. 1975. Natural cytotoxic reactivity of mouse lymphoid cells against syngeneic acid allogeneic tumors. I. Distribution of reactivity and specificity. Int J Cancer 16: 216-229.

31. Kärre, K., H. G. Ljunggren, G. Piontek, and R. Kiessling. 1986. Selective rejection of H-2-deficient lymphoma variants suggests alternative immune defence strategy. Nature 319: 675-678.

32. Trinchieri, G., and D. Santoli. 1978. Anti-viral activity induced by culturing lymphocytes with tumor-derived or virus-transformed cells. Enhancement of human natural killer cell activity by interferon and antagonistic inhibition of susceptibility of target cells to lysis. J Exp Med 147: 1314-1333.

33. Santoli, D., G. Trinchieri, and F. S. Lief. 1978. Cell-mediated cytotoxicity against virus-infected target cells in humans. I. Characterization of the effector lymphocyte. $J$ Immunol 121: 526-531.

34. Biron, C. A., K. B. Nguyen, G. C. Pien, L. P. Cousens, and T. P. Salazar-Mather. 1999. Natural killer cells in antiviral defense: function and regulation by innate cytokines. Annu Rev Immunol 17: 189-220.

35. Biron, C. A., K. S. Byron, and J. L. Sullivan. 1989. Severe herpesvirus infections in an adolescent without natural killer cells. N Engl J Med 320: 1731-1735.

36. Orange, J. S. 2006. Human natural killer cell deficiencies. Curr Opin Allergy Clin Immunol 6: 399-409.

37. Orange, J. S. 2002. Human natural killer cell deficiencies and susceptibility to infection. Microbes Infect 4: 1545-1558.

38. Chalmer, J. E., J. S. Mackenzie, and N. F. Stanley. 1977. Resistance to murine cytomegalovirus linked to the major histocompatibility complex of the mouse. J Gen Virol 37: 107-114.

39. Grundy, J. E., J. S. Mackenzie, and N. F. Stanley. 1981. Influence of H-2 and non-H2 genes on resistance to murine cytomegalovirus infection. Infect Immun 32: 277-286.

40. Bancroft, G. J., G. R. Shellam, and J. E. Chalmer. 1981. Genetic influences on the augmentation of natural killer (NK) cells during murine cytomegalovirus infection: correlation with patterns of resistance. J Immunol 126: 988-994.

41. Quinnan, G. V., and J. F. Manischewitz. 1987. Genetically determined resistance to lethal murine cytomegalovirus infection is mediated by interferon-dependent and independent restriction of virus replication. J Virol 61: 1875-1881.

42. Bukowski, J. F., B. A. Woda, and R. M. Welsh. 1984. Pathogenesis of murine cytomegalovirus infection in natural killer cell-depleted mice. J Virol 52: 119-128.

43. Béziat, V., L. L. Liu, J. A. Malmberg, M. A. Ivarsson, E. Sohlberg, A. T. Björklund, C. Retière, E. Sverremark-Ekström, J. Traherne, P. Ljungman, M. Schaffer, D. A. Price, 
J. Trowsdale, J. Michaëlsson, H. G. Ljunggren, and K. J. Malmberg. 2013. NK cell responses to cytomegalovirus infection lead to stable imprints in the human KIR repertoire and involve activating KIRs. Blood 121: 2678-2688.

44. Sun, J. C., J. N. Beilke, and L. L. Lanier. 2009. Adaptive immune features of natural killer cells. Nature 457: 557-561.

45. Scalzo, A. A., N. A. Fitzgerald, A. Simmons, A. B. La Vista, and G. R. Shellam. 1990. Cmv-1, a genetic locus that controls murine cytomegalovirus replication in the spleen. $J$ Exp Med 171: 1469-1483.

46. Scalzo, A. A., N. A. Fitzgerald, C. R. Wallace, A. E. Gibbons, Y. C. Smart, R. C. Burton, and G. R. Shellam. 1992. The effect of the Cmv-1 resistance gene, which is linked to the natural killer cell gene complex, is mediated by natural killer cells. $J$ Immunol 149: 581-589.

47. Brown, M. G., A. O. Dokun, J. W. Heusel, H. R. Smith, D. L. Beckman, E. A. Blattenberger, C. E. Dubbelde, L. R. Stone, A. A. Scalzo, and W. M. Yokoyama. 2001. Vital involvement of a natural killer cell activation receptor in resistance to viral infection. Science 292: 934-937.

48. Smith, H. R., J. W. Heusel, I. K. Mehta, S. Kim, B. G. Dorner, O. V. Naidenko, K. Iizuka, H. Furukawa, D. L. Beckman, J. T. Pingel, A. A. Scalzo, D. H. Fremont, and W. M. Yokoyama. 2002. Recognition of a virus-encoded ligand by a natural killer cell activation receptor. Proc Natl Acad Sci US A 99: 8826-8831.

49. Arase, H., E. S. Mocarski, A. E. Campbell, A. B. Hill, and L. L. Lanier. 2002. Direct recognition of cytomegalovirus by activating and inhibitory NK cell receptors. Science 296: $1323-1326$.

50. Scalzo, A. A., M. Manzur, C. A. Forbes, M. G. Brown, and G. R. Shellam. 2005. NK gene complex haplotype variability and host resistance alleles to murine cytomegalovirus in wild mouse populations. Immunol Cell Biol 83: 144-149.

51. Voigt, V., C. A. Forbes, J. N. Tonkin, M. A. Degli-Esposti, H. R. Smith, W. M. Yokoyama, and A. A. Scalzo. 2003. Murine cytomegalovirus $\mathrm{m} 157$ mutation and variation leads to immune evasion of natural killer cells. Proc Natl Acad Sci U S A 100: 13483-13488.

52. Delano, M. L., and D. G. Brownstein. 1995. Innate resistance to lethal mousepox is genetically linked to the NK gene complex on chromosome 6 and correlates with early restriction of virus replication by cells with an NK phenotype. J Virol 69: 5875-5877.

53. Pereira, R. A., A. Scalzo, and A. Simmons. 2001. Cutting edge: a NK complex-linked locus governs acute versus latent herpes simplex virus infection of neurons. J Immunol 166: 5869-5873.

54. Nash, W. T., J. Teoh, H. Wei, A. Gamache, and M. G. Brown. 2014. Know Thyself: NK-Cell Inhibitory Receptors Prompt Self-Tolerance, Education, and Viral Control. Front Immunol 5: 175.

55. Lanier, L. L. 1998. NK cell receptors. Annu Rev Immunol 16: 359-393.

56. Long, E. O., H. S. Kim, D. Liu, M. E. Peterson, and S. Rajagopalan. 2013. 
Controlling natural killer cell responses: integration of signals for activation and inhibition. Аппи Rev Immunol 31: 227-258.

57. Yokoyama, W. M., and S. Kim. 2006. Licensing of natural killer cells by self-major histocompatibility complex class I. Immunol Rev 214: 143-154.

58. Fernandez, N. C., E. Treiner, R. E. Vance, A. M. Jamieson, S. Lemieux, and D. H. Raulet. 2005. A subset of natural killer cells achieves self-tolerance without expressing inhibitory receptors specific for self-MHC molecules. Blood 105: 4416-4423.

59. Kim, S., J. Poursine-Laurent, S. M. Truscott, L. Lybarger, Y. J. Song, L. Yang, A. R. French, J. B. Sunwoo, S. Lemieux, T. H. Hansen, and W. M. Yokoyama. 2005. Licensing of natural killer cells by host major histocompatibility complex class I molecules. Nature 436: 709-713.

60. Anfossi, N., P. André, S. Guia, C. S. Falk, S. Roetynck, C. A. Stewart, V. Breso, C. Frassati, D. Reviron, D. Middleton, F. Romagné, S. Ugolini, and E. Vivier. 2006. Human NK cell education by inhibitory receptors for MHC class I. Immunity 25: 331-342.

61. Yokoyama, W. M., and S. Kim. 2006. How do natural killer cells find self to achieve tolerance? Immunity 24: 249-257.

62. Viant, C., A. Fenis, G. Chicanne, B. Payrastre, S. Ugolini, and E. Vivier. 2014. SHP1-mediated inhibitory signals promote responsiveness and anti-tumour functions of natural killer cells. Nat Commun 5: 5108.

63. Brodin, P., T. Lakshmikanth, S. Johansson, K. Kärre, and P. Höglund. 2009. The strength of inhibitory input during education quantitatively tunes the functional responsiveness of individual natural killer cells. Blood 113: 2434-2441.

64. Joncker, N. T., N. C. Fernandez, E. Treiner, E. Vivier, and D. H. Raulet. 2009. NK cell responsiveness is tuned commensurate with the number of inhibitory receptors for self-MHC class I: the rheostat model. J Immunol 182: 4572-4580.

65. Boudreau, J. E., T. J. Mulrooney, J. B. Le Luduec, E. Barker, and K. C. Hsu. 2016. KIR3DL1 and HLA-B Density and Binding Calibrate NK Education and Response to HIV. J Immunol 196: 3398-3410.

66. Joncker, N. T., N. Shifrin, F. Delebecque, and D. H. Raulet. 2010. Mature natural killer cells reset their responsiveness when exposed to an altered MHC environment. $J$ Exp Med 207: 2065-2072.

67. Elliott, J. M., J. A. Wahle, and W. M. Yokoyama. 2010. MHC class I-deficient natural killer cells acquire a licensed phenotype after transfer into an MHC class Isufficient environment. $J$ Exp Med 207: 2073-2079.

68. Wei, H., W. T. Nash, A. P. Makrigiannis, and M. G. Brown. 2014. Impaired NK-cell education diminishes resistance to murine CMV infection. Eur J Immunol 44: 32733282.

69. Ebihara, T., A. H. Jonsson, and W. M. Yokoyama. 2013. Natural killer cell licensing in mice with inducible expression of MHC class I. Proc Natl Acad Sci U S A 110: E4232-7. 
70. Ardolino, M., C. S. Azimi, A. Iannello, T. N. Trevino, L. Horan, L. Zhang, W. Deng, A. M. Ring, S. Fischer, K. C. Garcia, and D. H. Raulet. 2014. Cytokine therapy reverses NK cell anergy in MHC-deficient tumors. J Clin Invest 124: 4781-4794.

71. Xie, X., M. D. Stadnisky, E. R. Coats, M. M. Ahmed Rahim, A. Lundgren, W. Xu, A. P. Makrigiannis, and M. G. Brown. 2010. MHC class I D(k) expression in hematopoietic and nonhematopoietic cells confers natural killer cell resistance to murine cytomegalovirus. Proc Natl Acad Sci U S A 107: 8754-8759.

72. Boudreau, J. E., X. R. Liu, Z. Zhao, A. Zhang, L. D. Shultz, D. L. Greiner, B. Dupont, and K. C. Hsu. 2016. Cell-Extrinsic MHC Class I Molecule Engagement Augments Human NK Cell Education Programmed by Cell-Intrinsic MHC Class I. Immunity 45: 280-291.

73. Martin, M. P., Y. Qi, X. Gao, E. Yamada, J. N. Martin, F. Pereyra, S. Colombo, E. E. Brown, W. L. Shupert, J. Phair, J. J. Goedert, S. Buchbinder, G. D. Kirk, A. Telenti, M. Connors, S. J. O'Brien, B. D. Walker, P. Parham, S. G. Deeks, D. W. McVicar, and M. Carrington. 2007. Innate partnership of HLA-B and KIR3DL1 subtypes against HIV-1. .

74. Alter, G., S. Rihn, K. Walter, A. Nolting, M. Martin, E. S. Rosenberg, J. S. Miller, M. Carrington, and M. Altfeld. 2009. HLA class I subtype-dependent expansion of KIR3DS1+ and KIR3DL1+ NK cells during acute human immunodeficiency virus type 1 infection. J Virol 83: 6798-6805.

75. Jost, S., and M. Altfeld. 2013. Control of human viral infections by natural killer cells. Аппи Rev Immunol 31: 163-194.

76. Scalzo, A. A., P. A. Lyons, N. A. Fitzgerald, C. A. Forbes, W. M. Yokoyama, and G. R. Shellam. 1995. Genetic mapping of Cmv1 in the region of mouse chromosome 6 encoding the NK gene complex-associated loci Ly49 and musNKR-P1. Genomics 27: $435-441$.

77. Xie, X., A. Dighe, P. Clark, P. Sabastian, S. Buss, and M. G. Brown. 2007. Deficient major histocompatibility complex-linked innate murine cytomegalovirus immunity in MA/My.L-H2b mice and viral downregulation of H-2k class I proteins. $J$ Virol 81: 229236.

78. Xie, X., M. D. Stadnisky, and M. G. Brown. 2009. MHC class I Dk locus and Ly49G2+ NK cells confer H-2k resistance to murine cytomegalovirus. J Immunol 182: $7163-7171$.

79. Desrosiers, M. P., A. Kielczewska, J. C. Loredo-Osti, S. G. Adam, A. P. Makrigiannis, S. Lemieux, T. Pham, M. B. Lodoen, K. Morgan, L. L. Lanier, and S. M. Vidal. 2005. Epistasis between mouse Klra and major histocompatibility complex class I loci is associated with a new mechanism of natural killer cell-mediated innate resistance to cytomegalovirus infection. Nat Genet 37: 593-599.

80. Makrigiannis, A. P., A. T. Pau, A. Saleh, R. Winkler-Pickett, J. R. Ortaldo, and S. K. Anderson. 2001. Class I MHC-binding characteristics of the 129/J Ly49 repertoire. $J$ Immunol 166: 5034-5043.

81. Kielczewska, A., M. Pyzik, T. Sun, A. Krmpotic, M. B. Lodoen, M. W. Munks, M. Babic, A. B. Hill, U. H. Koszinowski, S. Jonjic, L. L. Lanier, and S. M. Vidal. 2009. 
Ly49P recognition of cytomegalovirus-infected cells expressing H2-Dk and CMVencoded m04 correlates with the NK cell antiviral response. J Exp Med 206: 515-523.

82. Orr, M. T., W. J. Murphy, and L. L. Lanier. 2010. "Unlicensed" natural killer cells dominate the response to cytomegalovirus infection. Nat Immunol 11:321-327.

83. Forbes, C. A., A. A. Scalzo, M. A. Degli-Esposti, and J. D. Coudert. 2016. Ly49C Impairs NK Cell Memory in Mouse Cytomegalovirus Infection. J Immunol 197: 128140.

84. Achdout, H., I. Manaster, and O. Mandelboim. 2008. Influenza virus infection augments NK cell inhibition through reorganization of major histocompatibility complex class I proteins. $J$ Virol 82: 8030-8037.

85. Mahmoud, A. B., M. M. Tu, A. Wight, H. S. Zein, M. M. Rahim, S. H. Lee, H. S. Sekhon, E. G. Brown, and A. P. Makrigiannis. 2016. Influenza Virus Targets Class I MHC-Educated NK Cells for Immunoevasion. .

86. Joffre, O. P., E. Segura, A. Savina, and S. Amigorena. 2012. Cross-presentation by dendritic cells. Nat Rev Immunol 12: 557-569.

87. Crowley, M., K. Inaba, and R. M. Steinman. 1990. Dendritic cells are the principal cells in mouse spleen bearing immunogenic fragments of foreign proteins. $J$ Exp Med 172: 383-386.

88. Steinman, R. M., and M. C. Nussenzweig. 2002. Avoiding horror autotoxicus: the importance of dendritic cells in peripheral T cell tolerance. Proc Natl Acad Sci U S A 99: 351-358.

89. Dudek, A. M., S. Martin, A. D. Garg, and P. Agostinis. 2013. Immature, SemiMature, and Fully Mature Dendritic Cells: Toward a DC-Cancer Cells Interface That Augments Anticancer Immunity. Front Immunol 4: 438.

90. Dalod, M., R. Chelbi, B. Malissen, and T. Lawrence. 2014. Dendritic cell maturation: functional specialization through signaling specificity and transcriptional programming. EMBO J 33: 1104-1116.

91. Krebs, P., M. J. Barnes, K. Lampe, K. Whitley, K. S. Bahjat, B. Beutler, E. Janssen, and K. Hoebe. 2009. NK-cell-mediated killing of target cells triggers robust antigenspecific T-cell-mediated and humoral responses. Blood 113: 6593-6602.

92. Janssen, E., K. Tabeta, M. J. Barnes, S. Rutschmann, S. McBride, K. S. Bahjat, S. P. Schoenberger, A. N. Theofilopoulos, B. Beutler, and K. Hoebe. 2006. Efficient T cell activation via a Toll-Interleukin 1 Receptor-independent pathway. Immunity 24: 787799.

93. Ge, M. Q., A. W. Ho, Y. Tang, K. H. Wong, B. Y. Chua, S. Gasser, and D. M. Kemeny. 2012. NK cells regulate CD8+ T cell priming and dendritic cell migration during influenza A infection by IFN- $\gamma$ and perforin-dependent mechanisms. J Immunol 189: 2099-2109.

94. Gillespie, A. L., J. Teoh, H. Lee, J. Prince, M. D. Stadnisky, M. Anderson, W. Nash, C. Rival, H. Wei, A. Gamache, C. R. Farber, K. Tung, and M. G. Brown. 2016. Genomic Modifiers of Natural Killer Cells, Immune Responsiveness and Lymphoid Tissue 
Remodeling Together Increase Host Resistance to Viral Infection. PLoS Pathog 12: e1005419.

95. Fernandez, N. C., A. Lozier, C. Flament, P. Ricciardi-Castagnoli, D. Bellet, M. Suter, M. Perricaudet, T. Tursz, E. Maraskovsky, and L. Zitvogel. 1999. Dendritic cells directly trigger NK cell functions: cross-talk relevant in innate anti-tumor immune responses in vivo. Nat Med 5: 405-411.

96. Borg, C., A. Jalil, D. Laderach, K. Maruyama, H. Wakasugi, S. Charrier, B. Ryffel, A. Cambi, C. Figdor, W. Vainchenker, A. Galy, A. Caignard, and L. Zitvogel. 2004. NK cell activation by dendritic cells (DCs) requires the formation of a synapse leading to IL12 polarization in DCs. Blood 104: 3267-3275.

97. Walzer, T., M. Dalod, S. H. Robbins, L. Zitvogel, and E. Vivier. 2005. Natural-killer cells and dendritic cells: "l'union fait la force". Blood 106: 2252-2258.

98. Zitvogel, L., M. Terme, C. Borg, and G. Trinchieri. 2006. Dendritic cell-NK cell cross-talk: regulation and physiopathology. Curr Top Microbiol Immunol 298: 157-174.

99. Gerosa, F., B. Baldani-Guerra, C. Nisii, V. Marchesini, G. Carra, and G. Trinchieri. 2002. Reciprocal activating interaction between natural killer cells and dendritic cells. $J$ Exp Med 195: 327-333.

100. Piccioli, D., S. Sbrana, E. Melandri, and N. M. Valiante. 2002. Contact-dependent stimulation and inhibition of dendritic cells by natural killer cells. J Exp Med 195: 335341.

101. Ferlazzo, G., M. L. Tsang, L. Moretta, G. Melioli, R. M. Steinman, and C. Münz. 2002. Human dendritic cells activate resting natural killer (NK) cells and are recognized via the NKp30 receptor by activated NK cells. J Exp Med 195: 343-351.

102. Altfeld, M., L. Fadda, D. Frleta, and N. Bhardwaj. 2011. DCs and NK cells: critical effectors in the immune response to HIV-1. Nat Rev Immunol 11: 176-186.

103. Hsu, K. M., J. R. Pratt, W. J. Akers, S. I. Achilefu, and W. M. Yokoyama. 2009. Murine cytomegalovirus displays selective infection of cells within hours after systemic administration. J Gen Virol 90: 33-43.

104. Benedict, C. A., C. De Trez, K. Schneider, S. Ha, G. Patterson, and C. F. Ware. 2006. Specific remodeling of splenic architecture by cytomegalovirus. PLoS Pathog 2: e16.

105. Andrews, D. M., A. A. Scalzo, W. M. Yokoyama, M. J. Smyth, and M. A. DegliEsposti. 2003. Functional interactions between dendritic cells and NK cells during viral infection. Nat Immunol 4: 175-181.

106. Mitrović, M., J. Arapović, S. Jordan, N. Fodil-Cornu, S. Ebert, S. M. Vidal, A. Krmpotić, M. J. Reddehase, and S. Jonjić. 2012. The NK cell response to mouse cytomegalovirus infection affects the level and kinetics of the early CD8(+) T-cell response. J Virol 86: 2165-2175.

107. Andrews, D. M., C. E. Andoniou, F. Granucci, P. Ricciardi-Castagnoli, and M. A. Degli-Esposti. 2001. Infection of dendritic cells by murine cytomegalovirus induces functional paralysis. Nat Immunol 2: 1077-1084. 
108. Andoniou, C. E., S. L. van Dommelen, V. Voigt, D. M. Andrews, G. Brizard, C. Asselin-Paturel, T. Delale, K. J. Stacey, G. Trinchieri, and M. A. Degli-Esposti. 2005. Interaction between conventional dendritic cells and natural killer cells is integral to the activation of effective antiviral immunity. Nat Immunol 6: 1011-1019.

109. Andrews, D. M., M. J. Estcourt, C. E. Andoniou, M. E. Wikstrom, A. Khong, V. Voigt, P. Fleming, H. Tabarias, G. R. Hill, R. G. van der Most, A. A. Scalzo, M. J. Smyth, and M. A. Degli-Esposti. 2010. Innate immunity defines the capacity of antiviral T cells to limit persistent infection. $J$ Exp Med 207: 1333-1343.

110. Dalod, M., T. Hamilton, R. Salomon, T. P. Salazar-Mather, S. C. Henry, J. D. Hamilton, and C. A. Biron. 2003. Dendritic cell responses to early murine cytomegalovirus infection: subset functional specialization and differential regulation by interferon alpha/beta. $J$ Exp Med 197: 885-898.

111. Banks, T. A., S. Rickert, C. A. Benedict, L. Ma, M. Ko, J. Meier, W. Ha, K. Schneider, S. W. Granger, O. Turovskaya, D. Elewaut, D. Otero, A. R. French, S. C. Henry, J. D. Hamilton, S. Scheu, K. Pfeffer, and C. F. Ware. 2005. A lymphotoxin-IFNbeta axis essential for lymphocyte survival revealed during cytomegalovirus infection. $J$ Immunol 174: 7217-7225.

112. Russell, J. H., and T. J. Ley. 2002. Lymphocyte-mediated cytotoxicity. Annu Rev Immunol 20: 323-370.

113. Borghesi, L., and C. Milcarek. 2007. Innate versus adaptive immunity: a paradigm past its prime? Cancer Res 67: 3989-3993.

114. Blattman, J. N., R. Antia, D. J. Sourdive, X. Wang, S. M. Kaech, K. Murali-Krishna, J. D. Altman, and R. Ahmed. 2002. Estimating the precursor frequency of naive antigenspecific CD8 T cells. J Exp Med 195: 657-664.

115. Davis, M. M., and P. J. Bjorkman. 1988. T-cell antigen receptor genes and T-cell recognition. Nature 334: 395-402.

116. Williams, M. A., and M. J. Bevan. 2007. Effector and memory CTL differentiation. Annu Rev Immunol 25: 171-192.

117. Zhang, N., and M. J. Bevan. 2011. CD8(+) T cells: foot soldiers of the immune system. Immunity 35: 161-168.

118. Deguine, J., B. Breart, F. Lemaître, J. P. Di Santo, and P. Bousso. 2010. Intravital imaging reveals distinct dynamics for natural killer and CD8(+) T cells during tumor regression. Immunity 33: 632-644.

119. Breart, B., F. Lemaitre, S. Celli, and P. Bousso. 2008. Two-photon imaging of intratumoral $\mathrm{CD} 8+\mathrm{T}$ cell cytotoxic activity during adoptive $\mathrm{T}$ cell therapy in mice. $J$ Clin Invest 118: 1390-1397.

120. Mrass, P., H. Takano, L. G. Ng, S. Daxini, M. O. Lasaro, A. Iparraguirre, L. L. Cavanagh, U. H. von Andrian, H. C. Ertl, P. G. Haydon, and W. Weninger. 2006. Random migration precedes stable target cell interactions of tumor-infiltrating T cells. $J$ Exp Med 203: 2749-2761.

121. Stinchcombe, J. C., G. Bossi, S. Booth, and G. M. Griffiths. 2001. The 
immunological synapse of CTL contains a secretory domain and membrane bridges. Immunity 15: 751-761.

122. Wiedemann, A., D. Depoil, M. Faroudi, and S. Valitutti. 2006. Cytotoxic T lymphocytes kill multiple targets simultaneously via spatiotemporal uncoupling of lytic and stimulatory synapses. Proc Natl Acad Sci U S A 103: 10985-10990.

123. Halle, S., K. A. Keyser, F. R. Stahl, A. Busche, A. Marquardt, X. Zheng, M. Galla, V. Heissmeyer, K. Heller, J. Boelter, K. Wagner, Y. Bischoff, R. Martens, A. Braun, K. Werth, A. Uvarovskii, H. Kempf, M. Meyer-Hermann, R. Arens, M. Kremer, G. Sutter, M. Messerle, and R. Förster. 2016. In Vivo Killing Capacity of Cytotoxic T Cells Is Limited and Involves Dynamic Interactions and T Cell Cooperativity. Immunity 44: 233245.

124. Harty, J. T., and V. P. Badovinac. 2008. Shaping and reshaping CD8+ T-cell memory. Nat Rev Immunol 8: 107-119.

125. Cooper, M. A., J. M. Elliott, P. A. Keyel, L. Yang, J. A. Carrero, and W. M. Yokoyama. 2009. Cytokine-induced memory-like natural killer cells. Proc Natl Acad Sci US A 106: 1915-1919.

126. Paust, S., H. S. Gill, B. Z. Wang, M. P. Flynn, E. A. Moseman, B. Senman, M. Szczepanik, A. Telenti, P. W. Askenase, R. W. Compans, and U. H. von Andrian. 2010. Critical role for the chemokine receptor CXCR6 in NK cell-mediated antigen-specific memory of haptens and viruses. Nat Immunol 11: 1127-1135.

127. Cui, W., and S. M. Kaech. 2010. Generation of effector CD8+ T cells and their conversion to memory T cells. Immunol Rev 236: 151-166.

128. Lau, L. L., B. D. Jamieson, T. Somasundaram, and R. Ahmed. 1994. Cytotoxic Tcell memory without antigen. Nature 369: 648-652.

129. Tan, J. T., B. Ernst, W. C. Kieper, E. LeRoy, J. Sprent, and C. D. Surh. 2002. Interleukin (IL)-15 and IL-7 jointly regulate homeostatic proliferation of memory phenotype CD8+ cells but are not required for memory phenotype CD4+ cells. J Exp Med 195: 1523-1532.

130. Bradley, L. M., L. Haynes, and S. L. Swain. 2005. IL-7: maintaining T-cell memory and achieving homeostasis. Trends Immunol 26: 172-176.

131. Kaech, S. M., and E. J. Wherry. 2007. Heterogeneity and cell-fate decisions in effector and memory CD8+ T cell differentiation during viral infection. Immunity 27: $393-405$.

132. Jameson, S. C., and D. Masopust. 2009. Diversity in T cell memory: an embarrassment of riches. Immunity 31: 859-871.

133. Joshi, N. S., W. Cui, A. Chandele, H. K. Lee, D. R. Urso, J. Hagman, L. Gapin, and S. M. Kaech. 2007. Inflammation directs memory precursor and short-lived effector $\mathrm{CD} 8(+) \mathrm{T}$ cell fates via the graded expression of T-bet transcription factor. Immunity 27: 281-295.

134. Kaech, S. M., J. T. Tan, E. J. Wherry, B. T. Konieczny, C. D. Surh, and R. Ahmed. 2003. Selective expression of the interleukin 7 receptor identifies effector CD8 T cells 
that give rise to long-lived memory cells. Nat Immunol 4: 1191-1198.

135. Sarkar, S., V. Kalia, W. N. Haining, B. T. Konieczny, S. Subramaniam, and R. Ahmed. 2008. Functional and genomic profiling of effector CD8 T cell subsets with distinct memory fates. $J$ Exp Med 205: 625-640.

136. Huster, K. M., V. Busch, M. Schiemann, K. Linkemann, K. M. Kerksiek, H. Wagner, and D. H. Busch. 2004. Selective expression of IL-7 receptor on memory T cells identifies early CD40L-dependent generation of distinct CD8+ memory T cell subsets. Proc Natl Acad Sci U S A 101: 5610-5615.

137. Zhang, M., S. Byrne, N. Liu, Y. Wang, A. Oxenius, and P. G. Ashton-Rickardt. 2007. Differential survival of cytotoxic T cells and memory cell precursors. J Immunol 178: 3483-3491.

138. Hamann, D., P. A. Baars, M. H. Rep, B. Hooibrink, S. R. Kerkhof-Garde, M. R. Klein, and R. A. van Lier. 1997. Phenotypic and functional separation of memory and effector human CD8+ T cells. J Exp Med 186: 1407-1418.

139. Appay, V., P. R. Dunbar, M. Callan, P. Klenerman, G. M. Gillespie, L. Papagno, G. S. Ogg, A. King, F. Lechner, C. A. Spina, S. Little, D. V. Havlir, D. D. Richman, N. Gruener, G. Pape, A. Waters, P. Easterbrook, M. Salio, V. Cerundolo, A. J. McMichael, and S. L. Rowland-Jones. 2002. Memory CD8+ T cells vary in differentiation phenotype in different persistent virus infections. Nat Med 8: 379-385.

140. Rutishauser, R. L., and S. M. Kaech. 2010. Generating diversity: transcriptional regulation of effector and memory CD8 T-cell differentiation. Immunol Rev 235: 219233.

141. Kaech, S. M., and W. Cui. 2012. Transcriptional control of effector and memory CD8+ T cell differentiation. Nat Rev Immunol 12: 749-761.

142. Intlekofer, A. M., N. Takemoto, E. J. Wherry, S. A. Longworth, J. T. Northrup, V. R. Palanivel, A. C. Mullen, C. R. Gasink, S. M. Kaech, J. D. Miller, L. Gapin, K. Ryan, A. P. Russ, T. Lindsten, J. S. Orange, A. W. Goldrath, R. Ahmed, and S. L. Reiner. 2005. Effector and memory CD8+ T cell fate coupled by T-bet and eomesodermin. Nat Immunol 6: 1236-1244.

143. Banerjee, A., S. M. Gordon, A. M. Intlekofer, M. A. Paley, E. C. Mooney, T. Lindsten, E. J. Wherry, and S. L. Reiner. 2010. Cutting edge: The transcription factor eomesodermin enables CD8+ T cells to compete for the memory cell niche. .

144. Van Stipdonk, M. J., E. E. Lemmens, and S. P. Schoenberger. 2001. Naïve CTLs require a single brief period of antigenic stimulation for clonal expansion and differentiation. Nat Immunol 2: 423-429.

145. Lenschow, D. J., T. L. Walunas, and J. A. Bluestone. 1996. CD28/B7 system of T cell costimulation. Annu Rev Immunol 14: 233-258.

146. Watts, T. H. 2005. TNF/TNFR family members in costimulation of T cell responses. Annu Rev Immunol 23: 23-68.

147. Cox, M. A., S. M. Kahan, and A. J. Zajac. 2013. Anti-viral CD8 T cells and the cytokines that they love. Virology 435: 157-169. 
148. Croft, M. 2014. The TNF family in T cell differentiation and function--unanswered questions and future directions. Semin Immunol 26: 183-190.

149. Welten, S. P., A. Redeker, K. L. Franken, J. D. Oduro, F. Ossendorp, L. Čičin-Šain, C. J. Melief, P. Aichele, and R. Arens. 2015. The viral context instructs the redundancy of costimulatory pathways in driving $\mathrm{CD} 8(+) \mathrm{T}$ cell expansion. elife 4.

150. Humphreys, I. R., S. W. Lee, M. Jones, A. Loewendorf, E. Gostick, D. A. Price, C. A. Benedict, C. F. Ware, and M. Croft. 2010. Biphasic role of 4-1BB in the regulation of mouse cytomegalovirus-specific CD8(+) T cells. Eur J Immunol 40: 2762-2768.

151. Humphreys, I. R., A. Loewendorf, C. de Trez, K. Schneider, C. A. Benedict, M. W. Munks, C. F. Ware, and M. Croft. 2007. OX40 costimulation promotes persistence of cytomegalovirus-specific CD8 T Cells: A CD4-dependent mechanism. J Immunol 179: 2195-2202.

152. Penaloza-MacMaster, P., A. Ur Rasheed, S. S. Iyer, H. Yagita, B. R. Blazar, and R. Ahmed. 2011. Opposing effects of CD70 costimulation during acute and chronic lymphocytic choriomeningitis virus infection of mice. J Virol 85: 6168-6174.

153. Nolte, M. A., R. W. van Olffen, K. P. van Gisbergen, and R. A. van Lier. 2009. Timing and tuning of CD27-CD70 interactions: the impact of signal strength in setting the balance between adaptive responses and immunopathology. Immunol Rev 229: 216231.

154. Nolte, M. A., and R. A. van Lier. 2006. The price of the CD27-CD70 costimulatory axis: you can't have it all. $J$ Exp Med 203: 2405-2408.

155. Dong, H., N. A. Franklin, D. J. Roberts, H. Yagita, M. J. Glennie, and T. N. Bullock. 2012. CD27 stimulation promotes the frequency of IL-7 receptor-expressing memory precursors and prevents IL-12-mediated loss of CD8(+) T cell memory in the absence of CD4(+) T cell help. J Immunol 188: 3829-3838.

156. Dong, H., N. A. Franklin, S. B. Ritchea, H. Yagita, M. J. Glennie, and T. N. Bullock. 2015. CD70 and IFN-1 selectively induce eomesodermin or T-bet and synergize to promote CD8+ T-cell responses. Eur J Immunol 45: 3289-3301.

157. Salek-Ardakani, S., and M. Croft. 2010. Tumor necrosis factor receptor/tumor necrosis factor family members in antiviral CD8 T-cell immunity. J Interferon Cytokine Res 30: 205-218.

158. Sallusto, F., D. Lenig, R. Förster, M. Lipp, and A. Lanzavecchia. 1999. Two subsets of memory $\mathrm{T}$ lymphocytes with distinct homing potentials and effector functions. Nature 401: 708-712.

159. Jabbari, A., and J. T. Harty. 2006. Secondary memory CD8+ T cells are more protective but slower to acquire a central-memory phenotype. J Exp Med 203: 919-932.

160. Huster, K. M., M. Koffler, C. Stemberger, M. Schiemann, H. Wagner, and D. H. Busch. 2006. Unidirectional development of CD8+ central memory T cells into protective Listeria-specific effector memory T cells. Eur J Immunol 36: 1453-1464.

161. Wherry, E. J., V. Teichgräber, T. C. Becker, D. Masopust, S. M. Kaech, R. Antia, U. H. von Andrian, and R. Ahmed. 2003. Lineage relationship and protective immunity of 
memory CD8 T cell subsets. Nat Immunol 4: 225-234.

162. Van Faassen, H., M. Saldanha, D. Gilbertson, R. Dudani, L. Krishnan, and S. Sad. 2005. Reducing the stimulation of CD8+ T cells during infection with intracellular bacteria promotes differentiation primarily into a central (CD62LhighCD44high) subset. J Immunol 174: 5341-5350.

163. Thompson, E. A., L. K. Beura, C. E. Nelson, K. G. Anderson, and V. Vezys. 2016. Shortened Intervals during Heterologous Boosting Preserve Memory CD8 T Cell Function but Compromise Longevity. J Immunol .

164. Klenerman, P., and A. Oxenius. 2016. T cell responses to cytomegalovirus. Nat Rev Immunol 16: 367-377.

165. Lanzavecchia, A., and F. Sallusto. 2000. Dynamics of T lymphocyte responses: intermediates, effectors, and memory cells. Science 290: 92-97.

166. Lisnić, V. J., A. Krmpotić, and S. Jonjić. 2010. Modulation of natural killer cell activity by viruses. Curr Opin Microbiol 13: 530-539.

167. Abi-Rached, L., and P. Parham. 2005. Natural selection drives recurrent formation of activating killer cell immunoglobulin-like receptor and Ly49 from inhibitory homologues. J Exp Med 201: 1319-1332.

168. Quinnan, G. V., N. Kirmani, A. H. Rook, J. F. Manischewitz, L. Jackson, G. Moreschi, G. W. Santos, R. Saral, and W. H. Burns. 1982. Cytotoxic t cells in cytomegalovirus infection: HLA-restricted T-lymphocyte and non-T-lymphocyte cytotoxic responses correlate with recovery from cytomegalovirus infection in bonemarrow-transplant recipients. $N$ Engl J Med 307: 7-13.

169. Reddehase, M. J., and U. H. Koszinowski. 1984. Significance of herpesvirus immediate early gene expression in cellular immunity to cytomegalovirus infection. Nature 312: 369-371.

170. Reddehase, M. J., F. Weiland, K. Münch, S. Jonjic, A. Lüske, and U. H. Koszinowski. 1985. Interstitial murine cytomegalovirus pneumonia after irradiation: characterization of cells that limit viral replication during established infection of the lungs. J Virol 55: 264-273.

171. Polić, B., H. Hengel, A. Krmpotić, J. Trgovcich, I. Pavić, P. Luccaronin, S. Jonjić, and U. H. Koszinowski. 1998. Hierarchical and redundant lymphocyte subset control precludes cytomegalovirus replication during latent infection. J Exp Med 188: 10471054.

172. Jonjić, S., I. Pavić, B. Polić, I. Crnković, P. Lucin, and U. H. Koszinowski. 1994. Antibodies are not essential for the resolution of primary cytomegalovirus infection but limit dissemination of recurrent virus. J Exp Med 179: 1713-1717.

173. Reddehase, M. J. 2000. The immunogenicity of human and murine cytomegaloviruses. Current opinion in immunology 12: 390-396.

174. Krmpotic, A., I. Bubic, B. Polic, P. Lucin, and S. Jonjic. 2003. Pathogenesis of murine cytomegalovirus infection. Microbes Infect 5: 1263-1277. 
175. Steffens, H. P., S. Kurz, R. Holtappels, and M. J. Reddehase. 1998. Preemptive CD8 T-cell immunotherapy of acute cytomegalovirus infection prevents lethal disease, limits the burden of latent viral genomes, and reduces the risk of virus recurrence. $J$ Virol 72: $1797-1804$.

176. Jonjić, S., W. Mutter, F. Weiland, M. J. Reddehase, and U. H. Koszinowski. 1989. Site-restricted persistent cytomegalovirus infection after selective long-term depletion of CD4+ T lymphocytes. J Exp Med 169: 1199-1212.

177. Lucin, P., I. Pavić, B. Polić, S. Jonjić, and U. H. Koszinowski. 1992. Gamma interferon-dependent clearance of cytomegalovirus infection in salivary glands. $J$ Virol 66: 1977-1984.

178. Tessmer, M. S., E. C. Reilly, and L. Brossay. 2011. Salivary gland NK cells are phenotypically and functionally unique. PLoS Pathog 7: e1001254.

179. Thom, J. T., T. C. Weber, S. M. Walton, N. Torti, and A. Oxenius. 2015. The Salivary Gland Acts as a Sink for Tissue-Resident Memory CD8(+) T Cells, Facilitating Protection from Local Cytomegalovirus Infection. Cell Rep 13: 1125-1136.

180. Smith, C. J., S. Caldeira-Dantas, H. Turula, and C. M. Snyder. 2015. Murine CMV Infection Induces the Continuous Production of Mucosal Resident T Cells. Cell Rep 13: $1137-1148$.

181. Jonjić, S., M. Babić, B. Polić, and A. Krmpotić. 2008. Immune evasion of natural killer cells by viruses. Curr Opin Immunol 20: 30-38.

182. Jones, T. R., E. J. Wiertz, L. Sun, K. N. Fish, J. A. Nelson, and H. L. Ploegh. 1996. Human cytomegalovirus US3 impairs transport and maturation of major histocompatibility complex class I heavy chains. Proc Natl Acad Sci U S A 93: 1132711333.

183. Park, B., Y. Kim, J. Shin, S. Lee, K. Cho, K. Früh, S. Lee, and K. Ahn. 2004. Human cytomegalovirus inhibits tapasin-dependent peptide loading and optimization of the MHC class I peptide cargo for immune evasion. Immunity 20: 71-85.

184. Noriega, V. M., J. Hesse, T. J. Gardner, K. Besold, B. Plachter, and D. Tortorella. 2012. Human cytomegalovirus US3 modulates destruction of MHC class I molecules. Mol Immunol 51: 245-253.

185. Lehner, P. J., J. T. Karttunen, G. W. Wilkinson, and P. Cresswell. 1997. The human cytomegalovirus US6 glycoprotein inhibits transporter associated with antigen processing-dependent peptide translocation. Proc Natl Acad Sci U S A 94: 6904-6909.

186. Llano, M., M. Gumá, M. Ortega, A. Angulo, and M. López-Botet. 2003. Differential effects of US2, US6 and US11 human cytomegalovirus proteins on HLA class Ia and HLA-E expression: impact on target susceptibility to NK cell subsets. Eur J Immunol 33: 2744-2754.

187. Gewurz, B. E., E. W. Wang, D. Tortorella, D. J. Schust, and H. L. Ploegh. 2001. Human cytomegalovirus US2 endoplasmic reticulum-lumenal domain dictates association with major histocompatibility complex class I in a locus-specific manner. $J$ Virol 75: 5197-5204. 
188. Machold, R. P., E. J. Wiertz, T. R. Jones, and H. L. Ploegh. 1997. The HCMV gene products US11 and US2 differ in their ability to attack allelic forms of murine major histocompatibility complex (MHC) class I heavy chains. J Exp Med 185: 363-366.

189. Ziegler, H., R. Thale, P. Lucin, W. Muranyi, T. Flohr, H. Hengel, H. Farrell, W. Rawlinson, and U. H. Koszinowski. 1997. A mouse cytomegalovirus glycoprotein retains MHC class I complexes in the ERGIC/cis-Golgi compartments. Immunity 6: 57-66.

190. Reusch, U., W. Muranyi, P. Lucin, H. G. Burgert, H. Hengel, and U. H. Koszinowski. 1999. A cytomegalovirus glycoprotein re-routes MHC class I complexes to lysosomes for degradation. EMBO J 18: 1081-1091.

191. Kleijnen, M. F., J. B. Huppa, P. Lucin, S. Mukherjee, H. Farrell, A. E. Campbell, U. H. Koszinowski, A. B. Hill, and H. L. Ploegh. 1997. A mouse cytomegalovirus glycoprotein, gp34, forms a complex with folded class I MHC molecules in the ER which is not retained but is transported to the cell surface. EMBO J 16: 685-694.

192. Kavanagh, D. G., M. C. Gold, M. Wagner, U. H. Koszinowski, and A. B. Hill. 2001. The multiple immune-evasion genes of murine cytomegalovirus are not redundant: $\mathrm{m} 4$ and m152 inhibit antigen presentation in a complementary and cooperative fashion. $J$ Exp Med 194: 967-978.

193. Wagner, M., A. Gutermann, J. Podlech, M. J. Reddehase, and U. H. Koszinowski. 2002. Major histocompatibility complex class I allele-specific cooperative and competitive interactions between immune evasion proteins of cytomegalovirus. $J$ Exp Med 196: 805-816.

194. Pinto, A. K., M. W. Munks, U. H. Koszinowski, and A. B. Hill. 2006. Coordinated function of murine cytomegalovirus genes completely inhibits CTL lysis. J Immunol 177: $3225-3234$.

195. Babić, M., M. Pyzik, B. Zafirova, M. Mitrović, V. Butorac, L. L. Lanier, A. Krmpotić, S. M. Vidal, and S. Jonjić. 2010. Cytomegalovirus immunoevasin reveals the physiological role of "missing self" recognition in natural killer cell dependent virus control in vivo. $J$ Exp Med 207: 2663-2673.

196. Prod'homme, V., C. Griffin, R. J. Aicheler, E. C. Wang, B. P. McSharry, C. R. Rickards, R. J. Stanton, L. K. Borysiewicz, M. López-Botet, G. W. Wilkinson, and P. Tomasec. 2007. The human cytomegalovirus MHC class I homolog UL18 inhibits LIR1+ but activates LIR-1- NK cells. J Immunol 178: 4473-4481.

197. Cretney, E., M. A. Degli-Esposti, E. H. Densley, H. E. Farrell, N. J. Davis-Poynter, and M. J. Smyth. 1999. m144, a murine cytomegalovirus (MCMV)-encoded major histocompatibility complex class I homologue, confers tumor resistance to natural killer cell-mediated rejection. $J$ Exp Med 190: 435-444.

198. Kubota, A., S. Kubota, H. E. Farrell, N. Davis-Poynter, and F. Takei. 1999. Inhibition of NK cells by murine CMV-encoded class I MHC homologue m144. Cell Immunol 191: 145-151.

199. Natarajan, K., A. Hicks, J. Mans, H. Robinson, R. Guan, R. A. Mariuzza, and D. H. Margulies. 2006. Crystal structure of the murine cytomegalovirus MHC-I homolog m144. J Mol Biol 358: 157-171. 
200. Corbett, A. J., J. D. Coudert, C. A. Forbes, and A. A. Scalzo. 2011. Functional consequences of natural sequence variation of murine cytomegalovirus m157 for Ly49 receptor specificity and NK cell activation. J Immunol 186: 1713-1722.

201. O'Hara, G. A., S. P. Welten, P. Klenerman, and R. Arens. 2012. Memory T cell inflation: understanding cause and effect. Trends Immunol 33: 84-90.

202. Gillespie, G. M., M. R. Wills, V. Appay, C. O'Callaghan, M. Murphy, N. Smith, P. Sissons, S. Rowland-Jones, J. I. Bell, and P. A. Moss. 2000. Functional heterogeneity and high frequencies of cytomegalovirus-specific CD8(+) T lymphocytes in healthy seropositive donors. J Virol 74: 8140-8150.

203. Karrer, U., S. Sierro, M. Wagner, A. Oxenius, H. Hengel, U. H. Koszinowski, R. E. Phillips, and P. Klenerman. 2003. Memory inflation: continuous accumulation of antiviral CD8+ T cells over time. J Immunol 170: 2022-2029.

204. Seckert, C. K., M. Griessl, J. K. Büttner, S. Scheller, C. O. Simon, K. A. Kropp, A. Renzaho, B. Kühnapfel, N. K. Grzimek, and M. J. Reddehase. 2012. Viral latency drives "memory inflation": a unifying hypothesis linking two hallmarks of cytomegalovirus infection. Med Microbiol Immunol 201: 551-566.

205. Snyder, C. M., K. S. Cho, E. L. Bonnett, S. van Dommelen, G. R. Shellam, and A. B. Hill. 2008. Memory inflation during chronic viral infection is maintained by continuous production of short-lived, functional T cells. Immunity 29: 650-659.

206. Snyder, C. M., J. E. Allan, E. L. Bonnett, C. M. Doom, and A. B. Hill. 2010. Crosspresentation of a spread-defective MCMV is sufficient to prime the majority of virusspecific CD8+ T cells. PLoS ONE 5: e9681.

207. Snyder, C. M., K. S. Cho, E. L. Bonnett, J. E. Allan, and A. B. Hill. 2011. Sustained CD8+ T cell memory inflation after infection with a single-cycle cytomegalovirus. PLoS Pathog 7: e1002295.

208. Smith, C. J., H. Turula, and C. M. Snyder. 2014. Systemic hematogenous maintenance of memory inflation by MCMV infection. PLoS Pathog 10: e1004233.

209. Munks, M. W., K. S. Cho, A. K. Pinto, S. Sierro, P. Klenerman, and A. B. Hill. 2006. Four distinct patterns of memory CD8 T cell responses to chronic murine cytomegalovirus infection. J Immunol 177: 450-458.

210. Munks, M. W., A. K. Pinto, C. M. Doom, and A. B. Hill. 2007. Viral interference with antigen presentation does not alter acute or chronic CD8 T cell immunodominance in murine cytomegalovirus infection. J Immunol 178: 7235-7241.

211. Masopust, D., and L. J. Picker. 2012. Hidden memories: frontline memory T cells and early pathogen interception. J Immunol 188: 5811-5817.

212. Bekiaris, V., F. Gaspal, F. M. McConnell, M. Y. Kim, D. R. Withers, C. Sweet, G. Anderson, and P. J. Lane. 2009. NK cells protect secondary lymphoid tissue from cytomegalovirus via a CD30-dependent mechanism. Eur J Immunol 39: 2800-2808.

213. Mandaric, S., S. M. Walton, T. Rülicke, K. Richter, M. J. Girard-Madoux, B. E. Clausen, A. Zurunic, M. Kamanaka, R. A. Flavell, S. Jonjic, and A. Oxenius. 2012. IL-10 suppression of NK/DC crosstalk leads to poor priming of MCMV-specific CD4 T cells 
and prolonged MCMV persistence. PLoS Pathog 8: e1002846.

214. Mailliard, R. B., Y. I. Son, R. Redlinger, P. T. Coates, A. Giermasz, P. A. Morel, W. J. Storkus, and P. Kalinski. 2003. Dendritic cells mediate NK cell help for Th1 and CTL responses: two-signal requirement for the induction of NK cell helper function. $J$ Immunol 171: 2366-2373.

215. Whitmire, J. K., J. T. Tan, and J. L. Whitton. 2005. Interferon-gamma acts directly on CD8+ T cells to increase their abundance during virus infection. J Exp Med 201: 1053-1059.

216. Whitmire, J. K., N. Benning, and J. L. Whitton. 2005. Cutting edge: early IFNgamma signaling directly enhances primary antiviral CD4+ T cell responses. J Immunol 175: 5624-5628.

217. Whitmire, J. K., B. Eam, N. Benning, and J. L. Whitton. 2007. Direct interferongamma signaling dramatically enhances $\mathrm{CD} 4+$ and $\mathrm{CD} 8+\mathrm{T}$ cell memory. J Immunol 179: 1190-1197.

218. Kos, F. J., and E. G. Engleman. 1996. Role of natural killer cells in the generation of influenza virus-specific cytotoxic T cells. Cell Immunol 173: 1-6.

219. Martín-Fontecha, A., L. L. Thomsen, S. Brett, C. Gerard, M. Lipp, A. Lanzavecchia, and F. Sallusto. 2004. Induced recruitment of NK cells to lymph nodes provides IFNgamma for T(H)1 priming. Nat Immunol 5: 1260-1265.

220. Tarrio, M. L., S. H. Lee, M. F. Fragoso, H. W. Sun, Y. Kanno, J. J. O’Shea, and C. A. Biron. 2014. Proliferation conditions promote intrinsic changes in NK cells for an IL10 response. J Immunol 193: 354-363.

221. Lee, S. H., K. S. Kim, N. Fodil-Cornu, S. M. Vidal, and C. A. Biron. 2009. Activating receptors promote NK cell expansion for maintenance, IL-10 production, and CD8 T cell regulation during viral infection. J Exp Med 206: 2235-2251.

222. Maynard, C. L., and C. T. Weaver. 2008. Diversity in the contribution of interleukin-10 to T-cell-mediated immune regulation. Immunol Rev 226: 219-233.

223. Waggoner, S. N., R. T. Taniguchi, P. A. Mathew, V. Kumar, and R. M. Welsh. 2010. Absence of mouse 2B4 promotes NK cell-mediated killing of activated CD8+ T cells, leading to prolonged viral persistence and altered pathogenesis. J Clin Invest 120: $1925-1938$.

224. Su, H., K. Nguyen, T. Salazar-Mather, M. Ruzek, M. Dalod, and C. Biron. NKcell functions restrainT cell responses during viral infections. .

225. Narni-Mancinelli, E., B. N. Jaeger, C. Bernat, A. Fenis, S. Kung, A. De Gassart, S. Mahmood, M. Gut, S. C. Heath, J. Estellé, E. Bertosio, F. Vely, L. N. Gastinel, B. Beutler, B. Malissen, M. Malissen, I. G. Gut, E. Vivier, and S. Ugolini. 2012. Tuning of natural killer cell reactivity by NKp46 and Helios calibrates T cell responses. Science 335: 344-348.

226. Welsh, R. M., and S. N. Waggoner. 2013. NK cells controlling virus-specific T cells: Rheostats for acute vs. persistent infections. Virology 435: 37-45. 
227. Schuster, I. S., M. E. Wikstrom, G. Brizard, J. D. Coudert, M. J. Estcourt, M. Manzur, L. A. O'Reilly, M. J. Smyth, J. A. Trapani, G. R. Hill, C. E. Andoniou, and M. A. Degli-Esposti. 2014. TRAIL + NK cells control CD4+ T cell responses during chronic viral infection to limit autoimmunity. Immunity 41: 646-656.

228. Madera, S., M. Rapp, M. A. Firth, J. N. Beilke, L. L. Lanier, and J. C. Sun. 2016. Type I IFN promotes NK cell expansion during viral infection by protecting NK cells against fratricide. $J$ Exp Med 213: 225-233.

229. Orange, J. S., and Z. K. Ballas. 2006. Natural killer cells in human health and disease. Clin Immunol 118: 1-10.

230. Biron, C. A. 2012. Yet another role for natural killer cells: cytotoxicity in immune regulation and viral persistence. Proc Natl Acad Sci U S A 109: 1814-1815.

231. Alter, G., and M. Altfeld. 2011. Mutiny or scrutiny: NK cell modulation of DC function in HIV-1 infection. Trends Immunol 32: 219-224.

232. Prince, J., A. Lundgren, M. D. Stadnisky, W. T. Nash, A. Beeber, S. D. Turner, and M. G. Brown. 2013. Multiparametric analysis of host response to murine cytomegalovirus in MHC class I-disparate mice reveals primacy of Dk-licensed Ly49G2+ NK cells in viral control. J Immunol 191: 4709-4719.

233. Torti, N., S. M. Walton, K. M. Murphy, and A. Oxenius. 2011. Batf3 transcription factor-dependent DC subsets in murine CMV infection: differential impact on T-cell priming and memory inflation. Eur J Immunol 41: 2612-2618.

234. Arens, R., A. Loewendorf, A. Redeker, S. Sierro, L. Boon, P. Klenerman, C. A. Benedict, and S. P. Schoenberger. 2011. Differential B7-CD28 costimulatory requirements for stable and inflationary mouse cytomegalovirus-specific memory CD8 T cell populations. J Immunol 186: 3874-3881.

235. Welten, S. P., A. Redeker, K. L. Franken, C. A. Benedict, H. Yagita, F. M. Wensveen, J. Borst, C. J. Melief, R. A. van Lier, K. P. van Gisbergen, and R. Arens. 2013. CD27-CD70 costimulation controls T cell immunity during acute and persistent cytomegalovirus infection. J Virol 87: 6851-6865.

236. Hendriks, J., Y. Xiao, and J. Borst. 2003. CD27 promotes survival of activated T cells and complements CD28 in generation and establishment of the effector T cell pool. J Exp Med 198: 1369-1380.

237. Yang, F. C., K. Agematsu, T. Nakazawa, T. Mori, S. Ito, T. Kobata, C. Morimoto, and A. Komiyama. 1996. CD27/CD70 interaction directly induces natural killer cell killing activity. Immunology 88: 289-293.

238. Takeda, K., H. Oshima, Y. Hayakawa, H. Akiba, M. Atsuta, T. Kobata, K. Kobayashi, M. Ito, H. Yagita, and K. Okumura. 2000. CD27-mediated activation of murine NK cells. J Immunol 164: 1741-1745.

239. De Colvenaer, V., S. Taveirne, M. Delforche, M. De Smedt, B. Vandekerckhove, T. Taghon, L. Boon, J. Plum, and G. Leclercq. 2011. CD27-deficient mice show normal NK-cell differentiation but impaired function upon stimulation. Immunol Cell Biol 89: 803-811. 
240. Cook, C. H., L. Chen, J. Wen, P. Zimmerman, Y. Zhang, J. Trgovcich, Y. Liu, and J. X. Gao. 2009. CD28/B7-mediated co-stimulation is critical for early control of murine cytomegalovirus infection. Viral Immunol 22: 91-103.

241. Sanchez, P. J., J. A. McWilliams, C. Haluszczak, H. Yagita, and R. M. Kedl. 2007. Combined TLR/CD40 stimulation mediates potent cellular immunity by regulating dendritic cell expression of CD70 in vivo. J Immunol 178: 1564-1572.

242. McWilliams, J. A., P. J. Sanchez, C. Haluszczak, L. Gapin, and R. M. Kedl. 2010. Multiple innate signaling pathways cooperate with CD40 to induce potent, CD70dependent cellular immunity. Vaccine 28: 1468-1476.

243. Van Deusen, K. E., R. Rajapakse, and T. N. Bullock. 2010. CD70 expression by dendritic cells plays a critical role in the immunogenicity of CD40-independent, CD4+ T cell-dependent, licensed CD8+ T cell responses. J Leukoc Biol 87: 477-485.

244. Obar, J. J., E. R. Jellison, B. S. Sheridan, D. A. Blair, Q. M. Pham, J. M. Zickovich, and L. Lefrançois. 2011. Pathogen-induced inflammatory environment controls effector and memory CD8+ T cell differentiation. J Immunol 187: 4967-4978.

245. Gamadia, L. E., E. M. van Leeuwen, E. B. Remmerswaal, S. L. Yong, S. Surachno, P. M. Wertheim-van Dillen, I. J. Ten Berge, and R. A. Van Lier. 2004. The size and phenotype of virus-specific $\mathrm{T}$ cell populations is determined by repetitive antigenic stimulation and environmental cytokines. J Immunol 172: 6107-6114.

246. Munitic, I., M. Kuka, A. Allam, J. P. Scoville, and J. D. Ashwell. 2013. CD70 deficiency impairs effector CD8 T cell generation and viral clearance but is dispensable for the recall response to lymphocytic choriomeningitis virus. J Immunol 190: 11691179.

247. Huleatt, J. W., and L. Lefrançois. 1995. Antigen-driven induction of CD11c on intestinal intraepithelial lymphocytes and CD8+ T cells in vivo. J Immunol 154: 56845693.

248. Lin, Y., T. J. Roberts, V. Sriram, S. Cho, and R. R. Brutkiewicz. 2003. Myeloid marker expression on antiviral CD8+ T cells following an acute virus infection. Eur $J$ Immunol 33: 2736-2743.

249. Grewal, I. S., and R. A. Flavell. 1998. CD40 and CD154 in cell-mediated immunity. Annu Rev Immunol 16: 111-135.

250. Taraban, V. Y., T. F. Rowley, and A. Al-Shamkhani. 2004. Cutting edge: a critical role for CD70 in CD8 T cell priming by CD40-licensed APCs. J Immunol 173: 65426546.

251. Bullock, T. N., and H. Yagita. 2005. Induction of CD70 on dendritic cells through CD40 or TLR stimulation contributes to the development of CD8+ T cell responses in the absence of CD4+ T cells. J Immunol 174: 710-717.

252. Bennett, S. R., F. R. Carbone, F. Karamalis, J. F. Miller, and W. R. Heath. 1997. Induction of a CD8+ cytotoxic $\mathrm{T}$ lymphocyte response by cross-priming requires cognate CD4+ T cell help. J Exp Med 186: 65-70.

253. Vankayalapati, R., P. Klucar, B. Wizel, S. E. Weis, B. Samten, H. Safi, H. Shams, 
and P. F. Barnes. 2004. NK cells regulate CD8+ T cell effector function in response to an intracellular pathogen. J Immunol 172: 130-137.

254. Hendriks, J., L. A. Gravestein, K. Tesselaar, R. A. van Lier, T. N. Schumacher, and J. Borst. 2000. CD27 is required for generation and long-term maintenance of T cell immunity. Nat Immunol 1: 433-440.

255. Matter, M., B. Odermatt, H. Yagita, J. M. Nuoffer, and A. F. Ochsenbein. 2006. Elimination of chronic viral infection by blocking CD27 signaling. J Exp Med 203: 2145-2155.

256. Brown, M. G., and L. D. Erickson. 2015. Editorial: NK cell reaping of Tfh cells: reckless slaughter or sensible pruning? J Leukoc Biol 98: 139-142.

257. Denoeud, J., and M. Moser. 2011. Role of CD27/CD70 pathway of activation in immunity and tolerance. J Leukoc Biol 89: 195-203.

258. Salek-Ardakani, S., R. Flynn, R. Arens, H. Yagita, G. L. Smith, J. Borst, S. P. Schoenberger, and M. Croft. 2011. The TNFR family members OX40 and CD27 link viral virulence to protective T cell vaccines in mice. J Clin Invest 121: 296-307.

259. Chen, L., and D. B. Flies. 2013. Molecular mechanisms of T cell co-stimulation and co-inhibition. Nat Rev Immunol 13: 227-242.

260. Sun, J. C., and L. L. Lanier. 2011. NK cell development, homeostasis and function: parallels with $\mathrm{CD}^{+} \mathrm{T}$ cells. Nat Rev Immunol 11: 645-657.

261. Mavilio, D., G. Lombardo, A. Kinter, M. Fogli, A. La Sala, S. Ortolano, A. Farschi, D. Follmann, R. Gregg, C. Kovacs, E. Marcenaro, D. Pende, A. Moretta, and A. S. Fauci. 2006. Characterization of the defective interaction between a subset of natural killer cells and dendritic cells in HIV-1 infection. $J$ Exp Med 203: 2339-2350.

262. Halenius, A., C. Gerke, and H. Hengel. 2015. Classical and non-classical MHC I molecule manipulation by human cytomegalovirus: so many targets — but how many arrows in the quiver? Cell Mol Immunol 12: 139-153.

263. Scalzo, A. A., A. J. Corbett, W. D. Rawlinson, G. M. Scott, and M. A. DegliEsposti. 2007. The interplay between host and viral factors in shaping the outcome of cytomegalovirus infection. Immunol Cell Biol 85: 46-54.

264. Pyzik, M., A. Kielczewska, and S. M. Vidal. 2008. NK cell receptors and their MHC class I ligands in host response to cytomegalovirus: insights from the mouse genome. Semin Immunol 20: 331-342.

265. Brune, W., C. Ménard, J. Heesemann, and U. H. Koszinowski. 2001. A ribonucleotide reductase homolog of cytomegalovirus and endothelial cell tropism. Science 291: 303-305.

266. Holtappels, R., J. Podlech, M. F. Pahl-Seibert, M. Jülch, D. Thomas, C. O. Simon, M. Wagner, and M. J. Reddehase. 2004. Cytomegalovirus misleads its host by priming of CD8 T cells specific for an epitope not presented in infected tissues. J Exp Med 199: $131-136$.

267. Gold, M. C., M. W. Munks, M. Wagner, U. H. Koszinowski, A. B. Hill, and S. P. 
Fling. 2002. The murine cytomegalovirus immunomodulatory gene m152 prevents recognition of infected cells by M45-specific CTL but does not alter the immunodominance of the M45-specific CD8 T cell response in vivo. J Immunol 169: 359-365.

268. Krmpotic, A., M. Messerle, I. Crnkovic-Mertens, B. Polic, S. Jonjic, and U. H. Koszinowski. 1999. The immunoevasive function encoded by the mouse cytomegalovirus gene m152 protects the virus against T cell control in vivo. $J$ Exp Med 190: $1285-1296$.

269. Cui, W., Y. Liu, J. S. Weinstein, J. Craft, and S. M. Kaech. 2011. An interleukin-21interleukin-10-STAT3 pathway is critical for functional maturation of memory CD8+ T cells. Immunity 35: 792-805.

270. Laidlaw, B. J., W. Cui, R. A. Amezquita, S. M. Gray, T. Guan, Y. Lu, Y.

Kobayashi, R. A. Flavell, S. H. Kleinstein, J. Craft, and S. M. Kaech. 2015. Production of IL-10 by CD4(+) regulatory T cells during the resolution of infection promotes the maturation of memory CD8(+) T cells. Nat Immunol 16: 871-879.

271. Blasius, A. L., E. Giurisato, M. Cella, R. D. Schreiber, A. S. Shaw, and M. Colonna. 2006. Bone marrow stromal cell antigen 2 is a specific marker of type I IFN-producing cells in the naive mouse, but a promiscuous cell surface antigen following IFN stimulation. J Immunol 177: 3260-3265.

272. Daley-Bauer, L. P., G. M. Wynn, and E. S. Mocarski. 2012. Cytomegalovirus impairs antiviral CD8 $+\mathrm{T}$ cell immunity by recruiting inflammatory monocytes. .

273. Daley-Bauer, L. P., L. J. Roback, G. M. Wynn, and E. S. Mocarski. 2014. Cytomegalovirus hijacks CX3CR1(hi) patrolling monocytes as immune-privileged vehicles for dissemination in mice. Cell Host Microbe 15: 351-362.

274. Carroll, V. A., A. Lundgren, H. Wei, S. Sainz, K. S. Tung, and M. G. Brown. 2012. Natural killer cells regulate murine cytomegalovirus-induced sialadenitis and salivary gland disease. J Virol 86: 2132-2142.

275. Lawson, C. M., J. E. Grundy, and G. R. Shellam. 1988. Antibody responses to murine cytomegalovirus in genetically resistant and susceptible strains of mice. $J$ Gen Virol 69 ( Pt 8): 1987-1998.

276. Söderberg-Nauclér, C., and J. Y. Nelson. 1999. Human cytomegalovirus latency and reactivation - a delicate balance between the virus and its host's immune system.

Intervirology 42: 314-321.

277. Vivier, E., E. Tomasello, M. Baratin, T. Walzer, and S. Ugolini. 2008. Functions of natural killer cells. Nat Immunol 9: 503-510.

278. Mathys, S., T. Schroeder, J. Ellwart, U. H. Koszinowski, M. Messerle, and U. Just. 2003. Dendritic cells under influence of mouse cytomegalovirus have a physiologic dual role: to initiate and to restrict T cell activation. J Infect Dis 187: 988-999.

279. Cerwenka, A., and L. L. Lanier. 2016. Natural killer cell memory in infection, inflammation and cancer. Nat Rev Immunol 16: 112-123.

280. Reeves, R. K., H. Li, S. Jost, E. Blass, H. Li, J. L. Schafer, V. Varner, C. Manickam, 
L. Eslamizar, M. Altfeld, U. H. von Andrian, and D. H. Barouch. 2015. Antigen-specific NK cell memory in rhesus macaques. Nat Immunol 16: 927-932.

281. Suarez-Ramirez, J. E., M. L. Tarrio, K. Kim, D. A. Demers, and C. A. Biron. 2014. CD8 T cells in innate immune responses: using STAT4-dependent but antigenindependent pathways to gamma interferon during viral infection. MBio 5: e01978e01914.

282. Lanier, L. L. 2008. Evolutionary struggles between NK cells and viruses. Nat Rev Immunol 8: 259-268.

283. Zheng, M., R. Sun, H. Wei, and Z. Tian. 2016. NK Cells Help Induce Anti-Hepatitis B Virus CD8+ T Cell Immunity in Mice. J Immunol 196: 4122-4131.

284. Chalupny, N. J., R. Peach, D. Hollenbaugh, J. A. Ledbetter, A. G. Farr, and A. Aruffo. 1992. T-cell activation molecule 4-1BB binds to extracellular matrix proteins. Proc Natl Acad Sci U S A 89: 10360-10364.

285. Wang, B., R. Maile, R. Greenwood, E. J. Collins, and J. A. Frelinger. 2000. Naive $\mathrm{CD} 8+\mathrm{T}$ cells do not require costimulation for proliferation and differentiation into cytotoxic effector cells. J Immunol 164: 1216-1222.

286. Shahinian, A., K. Pfeffer, K. P. Lee, T. M. Kündig, K. Kishihara, A. Wakeham, K. Kawai, P. S. Ohashi, C. B. Thompson, and T. W. Mak. 1993. Differential T cell costimulatory requirements in CD28-deficient mice. Science 261: 609-612.

287. Kündig, T. M., A. Shahinian, K. Kawai, H. W. Mittrücker, E. Sebzda, M. F. Bachmann, T. W. Mak, and P. S. Ohashi. 1996. Duration of TCR stimulation determines costimulatory requirement of T cells. Immunity 5: 41-52.

288. Cai, Z., and J. Sprent. 1996. Influence of antigen dose and costimulation on the primary response of CD8+ T cells in vitro. J Exp Med 183: 2247-2257.

289. Zimmermann, C., P. Seiler, P. Lane, and R. M. Zinkernagel. 1997. Antiviral immune responses in CTLA4 transgenic mice. J Virol 71: 1802-1807.

290. Corse, E., R. A. Gottschalk, and J. P. Allison. 2011. Strength of TCR-peptide/MHC interactions and in vivo T cell responses. J Immunol 186: 5039-5045.

291. Hemmer, B., I. Stefanova, M. Vergelli, R. N. Germain, and R. Martin. 1998. Relationships among TCR ligand potency, thresholds for effector function elicitation, and the quality of early signaling events in human T cells. J Immunol 160: 5807-5814.

292. Gottschalk, R. A., M. M. Hathorn, H. Beuneu, E. Corse, M. L. Dustin, G. AltanBonnet, and J. P. Allison. 2012. Distinct influences of peptide-MHC quality and quantity on in vivo T-cell responses. Proc Natl Acad Sci U S A 109: 881-886.

293. Prlic, M., G. Hernandez-Hoyos, and M. J. Bevan. 2006. Duration of the initial TCR stimulus controls the magnitude but not functionality of the CD8+ T cell response. $J$ Exp Med 203: 2135-2143.

294. Lipford, G. B., M. Hoffman, H. Wagner, and K. Heeg. 1993. Primary in vivo responses to ovalbumin. Probing the predictive value of the $\mathrm{Kb}$ binding motif. $J$ Immunol 150: $1212-1222$. 
295. Hogquist, K. A., S. C. Jameson, W. R. Heath, J. L. Howard, M. J. Bevan, and F. R. Carbone. 1994. T cell receptor antagonist peptides induce positive selection. Cell 76: 1727.

296. Pooley, J. L., W. R. Heath, and K. Shortman. 2001. Cutting edge: intravenous soluble antigen is presented to CD4 T cells by CD8- dendritic cells, but cross-presented to CD8 T cells by CD8+ dendritic cells. J Immunol 166: 5327-5330.

297. Pham, N. L., V. P. Badovinac, and J. T. Harty. 2009. A default pathway of memory CD8 $\mathrm{T}$ cell differentiation after dendritic cell immunization is deflected by encounter with inflammatory cytokines during antigen-driven proliferation. J Immunol 183: 23372348.

298. Katzenstein, D. A., G. S. Yu, and M. C. Jordan. 1983. Lethal infection with murine cytomegalovirus after early viral replication in the spleen. .

299. Torti, N., S. M. Walton, T. Brocker, T. Rülicke, and A. Oxenius. 2011. Nonhematopoietic cells in lymph nodes drive memory CD8 T cell inflation during murine cytomegalovirus infection. PLoS Pathog 7: e1002313.

300. Turner, D. L., K. L. Bickham, D. L. Farber, and L. Lefrançois. 2013. Splenic priming of virus-specific CD8 T cells following influenza virus infection. J Virol 87: 4496-4506.

301. Brinkmann, V., J. G. Cyster, and T. Hla. 2004. FTY720: sphingosine 1-phosphate receptor-1 in the control of lymphocyte egress and endothelial barrier function. Am J Transplant 4: 1019-1025.

302. Gallatin, W. M., I. L. Weissman, and E. C. Butcher. 2006. A cell-surface molecule involved in organ-specific homing of lymphocytes. 1983. J Immunol 177: 5-9.

303. Foulds, K. E., M. J. Rotte, and R. A. Seder. 2006. IL-10 is required for optimal CD8 T cell memory following Listeria monocytogenes infection. J Immunol 177: 2565-2574.

304. Ouyang, W., S. Rutz, N. K. Crellin, P. A. Valdez, and S. G. Hymowitz. 2011. Regulation and functions of the IL-10 family of cytokines in inflammation and disease. Annu Rev Immunol 29: 71-109.

305. Jost, N. H., S. Abel, M. Hutzler, T. Sparwasser, A. Zimmermann, A. Roers, W. Müller, R. Klopfleisch, H. Hengel, A. M. Westendorf, J. Buer, and W. Hansen. 2014. Regulatory T cells and T-cell-derived IL-10 interfere with effective anti-cytomegalovirus immune response. Immunol Cell Biol 92: 860-871.

306. Jones, M., K. Ladell, K. K. Wynn, M. A. Stacey, M. F. Quigley, E. Gostick, D. A. Price, and I. R. Humphreys. 2010. IL-10 restricts memory T cell inflation during cytomegalovirus infection. J Immunol 185: 3583-3592.

307. Perona-Wright, G., K. Mohrs, F. M. Szaba, L. W. Kummer, R. Madan, C. L. Karp, L. L. Johnson, S. T. Smiley, and M. Mohrs. 2009. Systemic but not local infections elicit immunosuppressive IL-10 production by natural killer cells. Cell Host Microbe 6: 503512.

308. Allam, A., M. Swiecki, W. Vermi, J. D. Ashwell, and M. Colonna. 2014. Dual function of CD70 in viral infection: modulator of early cytokine responses and activator 
of adaptive responses. J Immunol 193: 871-878.

309. Lenac Rovis, T., P. Kucan Brlic, N. Kaynan, V. Juranic Lisnic, I. Brizic, S. Jordan, A. Tomic, D. Kvestak, M. Babic, P. Tsukerman, M. Colonna, U. Koszinowski, M. Messerle, O. Mandelboim, A. Krmpotic, and S. Jonjic. 2016. Inflammatory monocytes and NK cells play a crucial role in DNAM-1-dependent control of cytomegalovirus infection. $J$ Exp Med .

310. Kamanaka, M., S. T. Kim, Y. Y. Wan, F. S. Sutterwala, M. Lara-Tejero, J. E. Galán, E. Harhaj, and R. A. Flavell. 2006. Expression of interleukin-10 in intestinal lymphocytes detected by an interleukin-10 reporter knockin tiger mouse. Immunity 25: 941-952.

311. Kim, M. T., S. P. Kurup, G. R. Starbeck-Miller, and J. T. Harty. 2016. Manipulating Memory CD8 T Cell Numbers by Timed Enhancement of IL-2 Signals. J Immunol .

312. Kotenko, S. V., S. Saccani, L. S. Izotova, O. V. Mirochnitchenko, and S. Pestka. 2000. Human cytomegalovirus harbors its own unique IL-10 homolog (cmvIL-10). Proc Natl Acad Sci U S A 97: 1695-1700.

313. Lockridge, K. M., S. S. Zhou, R. H. Kravitz, J. L. Johnson, E. T. Sawai, E. L. Blewett, and P. A. Barry. 2000. Primate cytomegaloviruses encode and express an IL-10like protein. Virology 268: 272-280.

314. Avdic, S., B. P. McSharry, M. Steain, E. Poole, J. Sinclair, A. Abendroth, and B. Slobedman. 2016. Human Cytomegalovirus-Encoded Human Interleukin-10 (IL-10) Homolog Amplifies Its Immunomodulatory Potential by Upregulating Human IL-10 in Monocytes. J Virol 90: 3819-3827.

315. Quinn, M., H. Turula, M. Tandon, B. Deslouches, T. Moghbeli, and C. M. Snyder. 2015. Memory T Cells Specific for Murine Cytomegalovirus Re-Emerge after Multiple Challenges and Recapitulate Immunity in Various Adoptive Transfer Scenarios. $J$ Immunol .

316. Nolz, J. C., and J. T. Harty. 2011. Protective capacity of memory CD8+ T cells is dictated by antigen exposure history and nature of the infection. Immunity 34: 781-793.

317. Joshi, N. S., W. Cui, C. X. Dominguez, J. H. Chen, T. W. Hand, and S. M. Kaech. 2011. Increased numbers of preexisting memory CD8 T cells and decreased T-bet expression can restrain terminal differentiation of secondary effector and memory CD8 T cells. J Immunol 187: 4068-4076.

318. Seder, R. A., P. A. Darrah, and M. Roederer. 2008. T-cell quality in memory and protection: implications for vaccine design. .

319. Sung, J. H., H. Zhang, E. A. Moseman, D. Alvarez, M. Iannacone, S. E. Henrickson, J. C. de la Torre, J. R. Groom, A. D. Luster, and U. H. von Andrian. 2012. Chemokine guidance of central memory $\mathrm{T}$ cells is critical for antiviral recall responses in lymph nodes. Cell 150: 1249-1263.

320. Alter, G., J. M. Malenfant, R. M. Delabre, N. C. Burgett, X. G. Yu, M. Lichterfeld, J. Zaunders, and M. Altfeld. 2004. Increased natural killer cell activity in viremic HIV-1 infection. J Immunol 173: 5305-5311.

321. Mavilio, D., G. Lombardo, J. Benjamin, D. Kim, D. Follman, E. Marcenaro, M. A. 
O'Shea, A. Kinter, C. Kovacs, A. Moretta, and A. S. Fauci. 2005. Characterization of CD56-/CD16+ natural killer (NK) cells: a highly dysfunctional NK subset expanded in HIV-infected viremic individuals. Proc Natl Acad Sci U S A 102: 2886-2891.

322. Wakeland, E., L. Morel, K. Achey, M. Yui, and J. Longmate. 1997. Speed congenics: a classic technique in the fast lane (relatively speaking). Immunol Today 18: 472-477.

323. Brown, M. G., A. A. Scalzo, L. R. Stone, P. Y. Clark, Y. Du, B. Palanca, and W. M. Yokoyama. 2001. Natural killer gene complex (Nkc) allelic variability in inbred mice: evidence for Nkc haplotypes. Immunogenetics 53: 584-591.

324. Lee, S. H., J. Gitas, A. Zafer, P. Lepage, T. J. Hudson, A. Belouchi, and S. M. Vidal. 2001. Haplotype mapping indicates two independent origins for the Cmv1s susceptibility allele to cytomegalovirus infection and refines its localization within the Ly49 cluster. Immunogenetics 53: 501-505.

325. Wheat, R. L., P. Y. Clark, and M. G. Brown. 2003. Quantitative measurement of infectious murine cytomegalovirus genomes in real-time PCR. J Virol Methods 112: 107113.

326. Kuka, M., I. Munitic, M. L. Giardino Torchia, and J. D. Ashwell. 2013. CD70 is downregulated by interaction with CD27. J Immunol 191: 2282-2289. 